Leandro Dias Cunha

Controlabilidade de Sistemas de Hardware para Computação Quântica: definição do problema e discussão de aspectos analíticos e numéricos

São Paulo 
Leandro Dias Cunha

\section{Controlabilidade de Sistemas de Hardware para Computação Quântica: definição do problema e discussão de aspectos analíticos e numéricos}

Tese apresentada à Escola Politécnica da Universidade de São Paulo para a obtenção do título de Doutor em Engenharia. Departamento de Engenharia de Telecomunicações e Controle

Orientador: José Roberto Castilho Piqueira

São Paulo

2016 
Catalogação-na-publicação

Cunha, Leandro Dias

Controlabilidade de Sistemas de Hardware para Computação Quântica: definição do problema e discussão de aspectos analíticos e numéricos. / L. D. Cunha -- São Paulo, 2016.

$219 \mathrm{p}$.

Tese (Doutorado) - Escola Politécnica da Universidade de São Paulo. Departamento de Engenharia de Telecomunicações e Controle.

1.Controle de sistemas 2.Controlabilidade 3.Sistemas quânticos 4.Medição em sistemas quânticos 5.Computação quântica I.Universidade de São Paulo. Escola Politécnica. Departamento de Engenharia de Telecomunicações e Controle II.t. 
À Ana Catarina. 


\section{Agradecimentos}

À Maria Isabel, por sua paciência, seu carinho e sua força. Aos familiares pela compreensão. À Ieda. Ao Professor Piqueira, pela qualidade e alcance de suas ideias e por sua sabedoria. Aos amigos. À Escola. À USP. 
"In the physicist's picture of quantum theory the notion of a real valued observable has, like Brahma, three visible faces : (i) a spectral measure on the line; (ii) a selfadjoint operator in a Hilbert space; (iii) a unitary representation of the real line as an additive group. The equivalence of these three descriptions is a consequence of von Neumann's spectral theorem for a, not necessarily bounded, selfadjoint operator and Stone's theorem on the infinitesimal generator of a one parameter unitary group in a Hilbert space."

Kalyanapuram Rangachari Parthasarathy. 


\section{Resumo}

Este trabalho possui como tema principal o estudo da dinâmica de sistemas quânticos da perspectiva da teoria de sistemas dinâmicos, em particular, do ponto de vista da teoria de controle. Os principais tópicos abordados são (i) a análise da controlabilidade dos sistemas quânticos em dimensão finita e infinita e (ii) a teoria generalizada de medição de sistemas quânticos com o objetivo de obter as equações diferenciais estocásticas associadas a sistemas submetidos a processos de medição contínuos. Com relação à controlabilidade de sistemas dinâmicos quânticos fechados em dimensão finita resgatamos da literatura os resultados, já consolidados, da aplicação da teoria de grupos e álgebras de Lie aos essa classe de sistemas dinâmicos. Em dimensão infinita, a aplicação direta das técnicas de controle geométrico já não ocorre diretamente. Em espaços de estados de dimensão infinita as técnicas de análise matemática devem ser mais sofisticadas, há problemas relacionados à convergência e problemas relacionados a operadores não limitados. Os principais resultados conhecidos da literatura são apresentados e suas limitações são discutidas. Realizamos em seguida uma analogia entre sistemas clássicos lineares e sistemas dinâmicos quânticos de dimensão infinita cuja dinâmica é restrita a uma álgebra de operadores auto adjuntos comutativa. Observamos também que a controlabilidade de alguns sistemas quânticos em dimensão infinita está associada a Hamiltonianos não lineares. Notamos, em particular, que os sistemas quânticos comutativos estão associados a operadores não lineares. Com relação à teoria de medição de sistemas quânticos, partimos da teoria de sistemas quânticos abertos para a obtenção da equação dinâmica que rege a evolução dos sistemas não conservativos. Em paralelo, realizamos uma análise da descrição matemáticas dos experimentos de medição em sistemas quânticos desde os postulados de medição ortogonal até a descrição de processos de medição contínuos. Observamos que a equação de Schrödinger estocástica associada a um processo de medição contínuo possui como gerador infinitesimal um Hamiltoniano não linear no operador auto adjunto associado ao observável. Realizamos em seguida uma discussão a respeito das implicações de processos de medição contínuos na dinâmica de sistemas quânticos, analisando possíveis impactos em sua controlabilidade. Analisamos também o caso particular de sistemas quânticos cujos operadores associados a sua dinâmica e a seus observáveis estão restritos a uma mesma álgebra comutativa. Concluímos com sugestões de trabalhos futuros relacionados controlabilidade em dimensão infinita e a à dinâmica de sistemas quânticos sujeitos a medição.

Palavras-chave: Sistemas de controle, controlabilidade, sistemas quânticos, sistemas quânticos abertos, medição em sistemas quânticos, filtragem quântica. 


\section{Abstract}

The main theme of this work is to study the dynamics of quantum systems from the perspective of the theory of dynamical systems, in particular, from the point of view of control theory. The main topics covered are (i) the analysis of controllability of quantum systems in finite and infinite dimensions and (ii) the general theory of measurement of quantum systems in order to get to the stochastic differential equations associated with systems subject to continuous measurement. Regarding the controllability of closed quantum dynamical systems in finite dimension, the standard results from the literature were presented: the application of group theory and Lie algebra to this class of dynamical systems. In infinite dimensions, the direct application of geometric control techniques is no longer possible. In infinite dimensional state spaces the mathematical analysis techniques need to be more sophisticated, there are problems related to convergence and issues related to unbounded operators. The main results known from the literature were presented and their limitations discussed. Then an analogy was performed between linear classical systems and infinite dimensional quantum dynamical systems whose dynamics is restricted to a commutative algebra of self adjoint operators. We also note that the controllability of some quantum systems in infinite dimension is associated with nonlinear Hamiltonians. We note, in particular, that the commutative quantum systems are associated with nonlinear operators. With respect to the measurement theory of quantum systems, we start in the structure of the theory of open quantum systems in order to obtain the dynamical equation governing the evolution of non-conservative systems. In parallel, we conducted an analysis of the mathematical description of the measurement experiments in quantum systems from the orthogonal measurement postulates to the description of continuous measurement. We noted that the stochastic Schrödinger equation associated with a continuous measurement process has as its infinitesimal generator a Hamiltonian nonlinear in the self-adjoint operator associated with the observable. Then a discussion about the implications of continuous measurement processes in the dynamics of quantum systems was conducted, analyzing possible impacts on its controllability. We also looked at the particular case of quantum systems whose operators associated with their dynamics and their observable are restricted to the same commutative algebra. We concluded with suggestions for future work related to controllability in infinite dimension and the dynamics of quantum systems subjected to measurement processes.

Keywords: Control systems, controllability, quantum systems, open quantum systems, quantum measurement, quantum filtering. 


\section{Lista de ilustrações}

Figura 1 - Lei de Schoelkopf . . . . . . . . . . . . . . . . . . . 47

Figura 2 - Medições fortes e fracas. Figura extraída de Breuer e Petruccione (BREUER; PETRUCCIONE, 2002). . . . . . . . . . . . . . 111

Figura 3 - Diagrama esquemático de um experimento de medição generalizada. Figura extraída de Breuer e Petruccione (BREUER; PETRUCCIONE, 2002) . . . . . . . . . . . . . . . . . . 116

Figura 4 - Representação esquemática de um experimento de medição indireta. Figura extraída de Breuer e Petruccione (BREUER; PETRUCCIONE, 2002). . . . . . . . . . . . . . . . . . . . . . . . . . . . . . . .

Figura 5 - Sistema incorporando medição do observável $J_{z}$. . . . . . . . . . . . 133

Figura 6 - Sinal obtido pela simulação de medição do observável $J_{z}$. . . . . . . . 134

Figura 7 - Aparato experimental. . . . . . . . . . . . . . . 134

Figura 8 - Valor esperado para os observáveis $J_{x}, J_{x}^{2}, J_{y}^{2}$ e $J_{z}^{2}$, no sistema com realimentação. . . . . . . . . . . . . . . . . . . 135

Figura 9 - Valor esperado para o observável $J_{x}$, desvio-padrão de $J_{x}$, $J_{y}$ e $J_{z}$ e parâmetro de squeezing, $\zeta$, no sistema com realimentação. . . . . . . . 136

Figura 10 - Amostra de trajetória para uma realização de ruído arbitrária, dinâmica livre.

Figura 11 - Amostra de trajetória para uma realização de ruído arbitrária, dinâmica livre.

Figura 12 - Amostra de trajetória para uma realização de ruído arbitrária, dinâmica livre.

Figura 13 - Amostra de trajetória para uma realização de ruído arbitrária, dinâmica controlada.

Figura 14 - Amostra de trajetória para uma realização de ruído arbitrária, dinâmica controlada. . . . . . . . . . . . . . . . . . . . . . 143

Figura 15 - Amostra de trajetória para uma realização de ruído arbitrária, dinâmica controlada.

Figura 16 - Amostra de trajetória para uma realização de ruído arbitrária, dinâmica controlada e função de controle modificada. . . . . . . . . . . . 145

Figura 17 - Amostra de trajetória para uma realização de ruído arbitrária, dinâmica controlada e função de controle modificada. . . . . . . . . . . . . 146

Figura 18 - Amostra de trajetória para uma realização de ruído arbitrária, dinâmica controlada e função de controle modificada. . . . . . . . . . . . . 147

Figura 19 - Dinâmica unitária . . . . . . . . . . . . . . . . . . 180

Figura 20 - Dinâmica dissipativa . . . . . . . . . . . . . . . . . . 181 
Figura 21 - Valores esperados para o qubit e para a cavidade . . . . . . . . . . . 182

Figura 22 - Função de Wigner da cavidade . . . . . . . . . . . . . . . . . . . . 183

Figura 23 - Trajetórias quânticas e sua dinâmica média . . . . . . . . . . . . . . 184

Figura 24 - Fidelidade de uma porta i-swap dissipativa . . . . . . . . . . . . . 186

Figura 25 - Oscilações de Rabi . . . . . . . . . . . . . . . . . . . . . . . 187

Figura 26 - Dinâmica dissipativa . . . . . . . . . . . . . . . . . . . . . 189

Figura 27 - Dinâmica não-dissipativa . . . . . . . . . . . . . . . . . . 190

Figura 28 - Probabilidade do estado excitado . . . . . . . . . . . . . . . . . . . 191

Figura 29 - Representação do modelo de Landau-Zener na esfera de Bloch . . . . . 192

Figura 30 - Quasienergias da dinâmica do modelo de Floquet . . . . . . . . . . . . 194

Figura 31 - Dinâmica da equação de Floquet-Markov . . . . . . . . . . . . . . . . . 195

Figura 32 - Dinâmica da equação de Bloch-Redfield. . . . . . . . . . . . . . . . 197

Figura 33 - QPT da dinâmica conservativa . . . . . . . . . . . . . . . . . . 199

Figura 34 - QPT da dinâmica dissipativa . . . . . . . . . . . . . . . 200

Figura 35 - Função de Wigner exemplificativa . . . . . . . . . . . . . . . . . . 201 


\section{Lista de tabelas}

Tabela 1 - Comparação entre diferentes sistemas quânticos, mostrando seus tem-

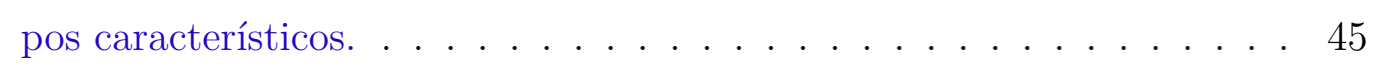

Tabela 2 - Quantum/Classical Analogies in State-Estimation . . . . . . . . . . 128 


\section{Sumário}

Introdução $\ldots \ldots \ldots \ldots \ldots \ldots \ldots \ldots \ldots$

FUNDAMENTOS E PERPECTIVA GERAL . . . . . . . . . . . . 17

1.1 Representação Matemática de Sistemas Dinâmicos . . . . . . . . 17

$1.2 \quad$ Controle de Sistemas Clássicos . . . . . . . . . . . . . . . 23

1.3 Dinâmica de Sistemas Quânticos . . . . . . . . . . . . . . . . . . 27

1.3.1 Formulação matemática em espaço de estados . . . . . . . . . . . 28

1.3.2 Formulação matemática por meio de operadores densidade . . . . . . . . 30

1.3.3 Princípio da Incerteza de Heisenberg e Processos de Medição . . . . . . . . 31

1.3.4 Medição de Sistemas Quânticos . . . . . . . . . . . . . . . . . . . 32

1.4 Controle de Sistemas Quânticos . . . . . . . . . . . . . . 32

1.5 Computação Quântica . . . . . . . . . . . . . 38

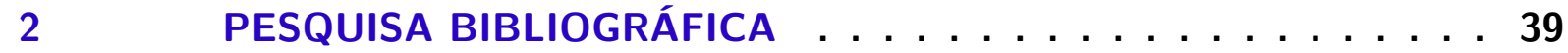

2.1 Do Processamento Quântico à Teoria de Controle . . . . . . . . . 41

2.2 Desafios Tecnológicos . . . . . . . . . . . . . . 45

$2.3 \quad$ Controlabilidade de sistemas quânticos . . . . . . . . . . . . 49

2.4 Medição, Filtragem e Controle . . . . . . . . . . . . . 53

$2.5 \quad$ Propostas de Hardware . . . . . . . . . . . . . . 55

3 CONTROLE DE SISTEMAS QUÂNTICOS EM MALHA ABERTA 57

3.1 Sistemas de controle lineares . . . . . . . . . . . 59

3.2 Controlabilidade de sistemas quânticos de dimensão finita . . . . . 61

3.3 Controlabilidade de Sistemas de dimensão infinita . . . . . . . . 65

3.4 Conclusão . . . . . . . . . . . . . . . . . . . . . . 85

4 CONTROLE DE SISTEMAS QUÂNTICOS EM MALHA FECHADA 88

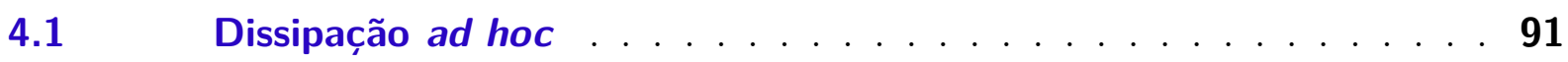

4.2 Equação de Redfield e Equações Mestras . . . . . . . . . . . . . . 94

4.2.1 Forma de Lindblad . . . . . . . . . . . . . . . . . . . . . . . 102

$4.3 \quad$ Decoerência . . . . . . . . . . . . . . . . . 105

4.4 Teoria da Medição Quântica . . . . . . . . . . . . . . . . . 110

4.5 Controle de Sistemas Quânticos Lineares . . . . . . . . . . . . . 130

$4.6 \quad$ Simulações Numéricas . . . . . . . . . . . . . . . . . . . 132

4.6 .1 Spin Squeezing via Realimentação . . . . . . . . . . . . . . . . . 132

$4.6 .2 \quad$ Controle de posição . . . . . . . . . . . . . . . . . . . . . . . . . 136 
RESULTADOS E DISCUSSÃO . . . . . . . . . . . . . . . 148

$5.1 \quad$ Incerteza e Medição . . . . . . . . . . . . . . . 148

5.2 Medição e Dinâmica . . . . . . . . . . . . . . . 150

5.3 Controlabilidade de Sistemas Quânticos . . . . . . . . . . . . . . 152

6 CONCLUSÕES E SUGESTÕES DE TRABALHOS FUTUROS $\ldots 155$

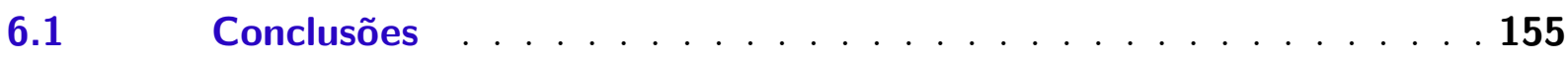

6.2 Sugestões de Trabalhos Futuros . . . . . . . . . . . . . . . 157

6.2.1 Caracterização da Controlabilidade em Dimensão Infinita . . . . . . . . . 157

6.2.2 Simulação de Filtros na esfera de Bloch . . . . . . . . . . . . . . . 163

6.2.3 Medição quântica e classificação dinâmica . . . . . . . . . . . . . . 166

Considerações finais $\ldots \ldots \ldots$. . . . . . . . . . . . . 167

Referências . . . . . . . . . . . . . . . 168

$\begin{array}{ll}\text { ANEXOS } & 178\end{array}$

ANEXO A - BILIOTECA DE SIMULAÇÃO NUMÉRICA - QUTIP 179

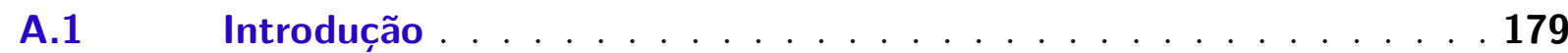

A.1.1 Dinâmica Conservativa . . . . . . . . . . . . . . . . . . . . . . 179

A.1.2 Dinâmica Dissipativa . . . . . . . . . . . . . . . . . . . 180

A.2 Exemplos da versão $1 \ldots \ldots \ldots 1$

A.2.1 Exemplo 01 - Valores esperados e ground-states . . . . . . . . . . . . 181

A.2.2 Exemplo 02 - Trajetorias quânticas . . . . . . . . . . . . . . . . . . . . 184

A.2.3 Exemplo 3 - Porta quântica - conservativa e dissipativa . . . . . . . . . 185

A.2.4 Exemplo 4 - Oscilações de Rabi . . . . . . . . . . . . . . . . . 187

A.2.5 Exemplo $5-3$ subsistemas . . . . . . . . . . . . . . . . . . 188

A.2.6 Exemplo 6 - Dinâmica do modelo de Landau-Zener . . . . . . . . . . . 190

A.2.7 Exemplo 7 - Representação na esfera de Bloch . . . . . . . . . . . . 192

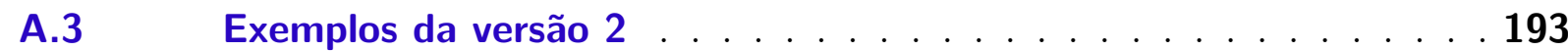

A.3.1 Exemplo 1 - Floquet . . . . . . . . . . . . . . . . . . . . 193

A.3.2 Exemplo 2 - Dinâmica Floquet . . . . . . . . . . . . . . . . . . 195

A.3.3 Exemplo 3 - Equação de Bloch-Redfield . . . . . . . . . . . . . . 196

A.3.4 Exemplo 5 - Quantum Process Tomography . . . . . . . . . . . . . . . 198

A.3.5 Exemplo 6 - Função de Wigner . . . . . . . . . . . . . . . . . . 200

ANEXO B - SISTEMAS DINÂMICOS . . . . . . . . 203

ANEXO C - CÓDIGO FONTE EM MATLAB . . . . . . 210 
ANEXO D - CÓDIGO FONTE EM QTIP . . . . . . . . 214

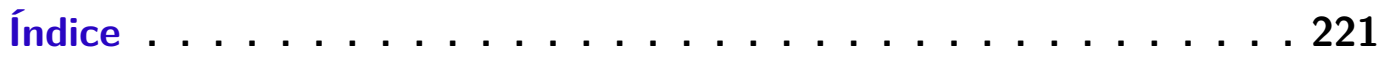




\section{Introdução}

O presente trabalho é desenvolvido na interface entre as áreas de pesquisa da computação quântica (NIELSEN; CHUANG, 2000) e da teoria matemática de controle (JURDJEVIC, 1997). A investigação científica sobre a utilização de sistemas dinâmicos de natureza e comportamento quânticos para processar informação, criando um sistema de computação dedicado, iniciou-se com a publicação do artigo "Simulating physics with computers" (FEYNMAN, 1982), no qual, ao discutir o custo computacional envolvido na simulação de sistemas quânticos em função da necessidade de manipulação intensa de matrizes, Feynman inverteu o sentido do raciocínio e mostrou que a dinâmica de um sistema quântico está relacionada a uma capacidade de processamento de informação equivalente à intensa manipulação matricial. Em seguida, no artigo "Quantum theory, the ChurchTuring principle and the universal quantum computer" (DEUTSCH, 1985), a partir das características intrínsecas aos sistemas quânticos, Deutsch idealizou uma máquina computacional de natureza quântica, utilizando superposição linear e estados emaranhados, e apresentou um algoritmo por meio do qual demonstrava a superioridade do computador quântico para uma determinada classe de problemas. O potencial de um sistema quântico de computação passou a ser de grande interesse prático a partir de 1994 com a publicação dos trabalhos de Peter Shor, nos quais ele apresentou um algoritmo quântico para fatoração em números primos e mostrou que a classe de complexidade computacional quântica para esse problema é polinomial (SHOR, 1994; SHOR, 1997). ${ }^{1}$ Em computadores clássicos o mesmo problema era resolvido de forma menos eficiente. No campo da codificação e criptografia em ciência da computação clássica, a segurança de alguns sistemas é baseada na dificuldade de se fatorar um número grande em fatores primos. Há algoritmos quânticos mais eficientes também para executar buscas e transformadas de Fourier. Este conjunto de fatores trouxe grande interesse na implementação física de um dispositivo computacional capaz de executar código quântico (NIELSEN; CHUANG, 2000).

O objetivo principal deste estudo é analisar a dinâmica de sistemas quânticos, buscando os pontos comuns e as principais diferenças com relação à dinâmica de sistemas clássicos. O trabalho estabelece como focos específicos de investigação a análise da controlabilidade e a análise dos processos de medição quânticos. É também um objetivo geral do trabalho analisar as áreas de pesquisa associadas à computação quântica do ponto de vista da engenharia, em especial do ponto de vista da engenharia de sistemas e controle.

Buscaremos ilustrar os modelos matemáticos desenvolvidos na área de controle de sistemas quânticos por realimentação apresentando os resultados de simulações numéricas

1 Um algoritmo clássico de classe de complexidade polinomial foi recentemente obtido (AGRAWAL; KAYAL; SAXENA, 2004) apud (MARTINEZ et al., 2015). 
desenvolvidas no âmbito do trabalho. Nas simulações reproduzimos de forma parcial dois modelos encontrados na literatura, provenientes de dois melhores grupos de pesquisa do mundo na área de dinâmica e controle de sistemas quânticos.

Neste trabalho não analisamos algumas questão fundamentais para a computação quântica ou processamento quântico de informação que podem trazer para a área de sistemas e controle situações com configurações diferentes ou até mesmo problemas novos. No capítulo 3, no qual focamos a questão da controlabilidade, não analisamos a forma como a informação é codificada no estado quântico, nem analisamos diretamente como essa informação é dinamicamente processada. Não foi investigado, portanto, se existem situações ou condições de maior susceptibilidade do estado quântico estático ou de sua dinâmica. Analisamos, no entanto, de forma qualitativa os processos de dissipação e de decoerência como motivações e pré-requisitos para uma teoria quântica de medição generalizada. Esta interface que acabamos de descrever, que investiga o vazamento de energia e de informação na interface entre o sistema e seu ambiente, é uma importante área de pesquisa que estuda os processos de ocorrência de erros e busca tornar o processamento quântico de informação mais robusto tanto por meio da construção de hardware menos susceptível ao ambiente, quanto por meio da especificação de uma arquitetura de hardware redundante que se torna tolerante a uma certa taxa de falhas de hardware.

Não analisamos também a geração e a manipulação dos estados quânticos emaranhados do ponto de vista da dinâmica de sistemas e controle. Chegamos a abordar importantes aspectos dinâmicos relacionados ao emaranhamento como a decoerência e algumas particularidades dos processos de medição generalizados que estão a ela vinculados. Dessa forma, não analisamos o emaranhamento como "recurso", mas apenas como fonte de fenômenos potencialmente problemáticos e como fundamento para explicar e justificar a teoria geral de medição.

Também não desenvolvemos as questões locais e globais relacionadas à busca de trajetórias ótimas de controle no espaço de estados. Por problemas de controle ótimo de natureza local, nos referimos ao desenho de trajetórias dinâmicas que interligam poucos bits quânticos (subsistemas de dois níveis denominados qubits). Esses problemas estão associados ao desenho de portas lógicas quânticas. Um conhecido resultado da computação quântica informa que muitos conjuntos finitos de portas lógicas são universais, o que quer dizer que qualquer operador de evolução em $S U(n)$ pode ser construído a partir da síntese de um circuito quântico adequado cujos componentes são elementos de um tal conjunto universal de portas lógicas. De forma similar, as operações NAND e NOR são universais na lógica booleana. Assim, por exemplo, dada uma especificação de hardware, uma análise local poderia definir o melhor conjunto universal a ser construído a partir do material físico.

Por problemas de controle ótimo globais, nos referimos ao desenho de trajetórias 
que executam algoritmos, desde a inicialização de variáveis até a obtenção do resultado final. Desta perspectiva se poderia realizar, por exemplo, comparações entre diferentes conjuntos universais na implementação de um algoritmo, dada uma plataforma de hardware.

A universalidade de conjuntos de portas lógicas é um conceito equivalente à controlabilidade. Assim, a partir de um sistema dinâmico quântico controlável é possível sintetizar um conjunto de portas lógicas de tal forma que um computar quântico universal pode ser a partir delas contruído. 


\section{Fundamentos e perpectiva geral}

A finalidade deste capítulo é estabelecer, a partir de uma linguagem matemática padronizada, os fundamentos mínimos necessários da mecânica quântica e da teoria de sistemas e controle para definir objetivamente a área da computação quântica ${ }^{1}$. Algumas definições e resultados adicionais da teoria de sistemas dinâmicos podem ser encontrados no Anexo B. Os conceitos e resultados relacionados à mecânica quântica podem ser consultados na imensa literatura disponível sobre o tema, por exemplo (COHEN-TANNOUDJI; DIU; LALOë, 1977). Em seguida, a partir dos fundamentos estabelecidos, buscar-seá definir o que é um computador quântico apresentando modelos para seu hardware. Os aspectos que serão representados nos modelos buscam caracterizar um computador quântico tanto do ponto de vista da ciência da computação e da física, quanto, mais detalhadamente, da perspectiva da engenharia de sistemas. (MILNE; KOROLKOVA, 2012) (BARNES; SARMA, 2012) (KIESSLICH et al., 2011)

\subsection{Representação Matemática de Sistemas Dinâmicos}

O conceito de Sistema Dinâmico é abrangente uma vez que deve alcançar qualquer problema de evolução temporal de estados físicos, envolvendo tanto sistemas discretos quanto sistemas contínuos. A linguagem de grupos de transformações a um parâmetro, a ser descrita abaixo, é bem adequada para descrever fenômenos evolutivos. No caso de sistemas contínuos, sua evolução é normalmente descrita por uma equação diferencial.

A teoria de equações diferenciais ordinárias, EDO's, é uma ferramenta fundamental na ciência. Através de seus métodos, estabelecem-se modelos matemáticos de evolução de sistemas e suas consequências podem exploradas. Ao se tornar um paradigma de estudo científico, um modelo é constantemente testado contra resultados experimentais. Quando os modelos apresentam pouca aderência aos resultados experimentais, são aperfeiçoados e surge um novo paradigma científico (KUHN, 1970) apud (CHALMERS, 1993). É possível modelar processos evolutivos determinísticos e diferenciáveis em dimensão finita. Um processo é determinístico se todo o seu curso futuro e também o seu passado são unicamente determinados pelo seu estado em um instante fixo. O conjunto de todos os estados possíveis de um processo é chamado espaço de fases. Quando o espaço de fases de um processo é um espaço de dimensão finita, esse processo é também chamado de dimensão finita. Um processo é chamado diferenciável quando seu espaço de fases possui a estrutura de uma variedade diferenciável e a lei de mudança de estado com o tempo é diferenciável.

1 A computação quântica é utilizada neste trabalho como principal alvo para a aplicação das técnicas de controle de sistemas quânticos. Trata-se, portanto, de um objetivo geral 
O estudo das equações diferenciais está intimamente relacionado ao progresso teórico na física. Na mecânica, Newton escreveu as equações diferenciais ordinárias que regem o movimento dos corpos rígidos e as usou, juntamente com sua teoria de gravitação, para explicar os fenômenos celestes com grande acerto. Mais que isso, Newton estabeleceu definitivamente a matemática e a geometria como a linguagem de descrição da natureza e as equações diferenciais como a expressão do determinismo científico. A solução de uma equação diferencial fornece a evolução temporal de um sistema físico dadas apenas suas condições iniciais. Em condições especiais as soluções podem existir para todo instante $t \in \Re$. Esse resultado levou Laplace a acreditar no determinismo completo do universo, ou seja, na ausência de incerteza (HALL, 1994). Laplace chegou a demonstrar matematicamente a estabilidade do sistema solar. Mais tarde, no entanto, em um exame matemático mais criterioso, Poincare mostrou que a estabilidade era indeterminada uma vez que o sistema era "caótico".

Para tanto, Poincare criou a teoria qualitativa das equações diferenciais. A teoria das equações diferenciais era formada por métodos de solução de equações específicas ou por métodos de aproximar as soluções verdadeiras. O fato de uma grande classe de equações não possuirem solução explícita incomodava bastante os físicos. A teoria qualitativa busca encontrar informações a respeito do comportamento das soluções das equações sem ter que resolvê-las para tanto. Tal estudo se mostrou bastante rico e fundamental uma vez que seu desenvolvimento levou Poincare a investigar mais profundamente as propriedades dos espaços onde as equações estavam definidas. Estes espaços são geralmente $\Re^{n}$, para algum $n$ finito, ou superfícies definidas nesses espaços. O estudo desses espaços levou ao desenvolvimento da topologia, área da matemática que investiga diversas propriedades de subconjuntos de um espaço que são determinantes de características importantes como continuidade e convergência, por exemplo.

Deve-se nesse ponto destacar alguns fundamentos sobre os quais as teorias físicas evoluíram até o início do século XX. A partir do estrondoso sucesso da teoria mecânica de Isaac Newton e do espetacular desenvolvimento da matemática que se seguiu, a crença no determinismo científico passou a ser, ainda que indiretamente, assumida pelos cientistas. Acreditava-se que os erros experimentais eram atribuíveis em grande parte à imperfeição dos aparatos de medição. Por esse paradigma, fossem os cientistas dotados de processos de medição cada vez mais refinados, os modelos poderiam ter seus parâmetros aperfeiçoados, os resultados experimentais apresentariam incertezas cada vez menores e, no limite, o modelo ideal seria obtido e teria sua validade confirmada experimentalmente. Como consequência, obtidos o modelo ideal de um sistema qualquer e uma descrição completa de seu estado físico, toda a evolução temporal do sistema poderia ser obtida para qualquer

$\overline{2}$ Abertos de $\Re^{n}$ possuem naturalmente uma estrutura de variedade diferenciável de classe $C^{\infty}$ 
instante de tempo passado ou futuro. Essa é a expressão do determinismo. Outra questão importante a ser destacada é que o espaço de fases era diretamente relacionado aos parâmetros físicos que poderiam ser experimentalmente medidos. Interessa-se, em processos de modelagem clássicos, por definir um conjunto de parâmetros físicos capazes de variar de forma independente, delimitando um espaço de fases. O número dessas variáveis representadas por grandezas linearmente independentes define a dimensão do sistema e na maior parte das vezes, essas variáveis físicas são diretamente observáveis ou são dependentes de variáveis que podem ser diretamente observadas. A capacidade de se observar fisicamente um domínio da realidade e a determinação do espaço de fases do respectivo modelo matemático são questões interdependentes que muitas vezes se confundem no tratamento de sistemas clássicos. Tanto o determinismo quanto a capacidade irrestrita de observação física universais foram traumaticamente retirados das ciências naturais com o advento da mecânica quântica no começo do século XX. ${ }^{3}$

A capacidade de se observar sistemas físicos de acordo com o paradigma estabelecido pela física clássica é um dos fundamentos epistemológicos do modelo racional que fazemos da realidade. Nossa intuição (instinto) a respeito do mundo físico que nos cerca foi desenvolvida com base nesse paradigma. Os seres humanos nascem dotados de sentidos capazes de captar aspectos da realidade e de um cérebro capaz de manipulá-los logicamente e de fazer previsões e antecipações a respeito do mundo. Pode-se afirmar que a ciência natural evoluiu por meio de construções lógicas cada vez mais complexas relacionando fatos da realidade que por sua vez são dependendentes de processos cognitivos básicos e de observações diretas da natureza por meio dos sentidos. A verdade, ou validade, de uma inferência lógica sempre foram testáveis em face da realidade física por meio de observação com o uso dos sentidos. A partir de um modelo matemático da realidade física todas as proposições relativas ao estado do sistema em um instante qualquer poderiam ser exaustivamente testadas e ter um valor de verdade atribuído de forma absoluta (ou uma proposição é completamente verdadeira ou completamente falsa, mesmo que essa proposição seja a respeito de uma distribuição de probabilidade). ${ }^{4}$

A forma pela qual a física quântica modela os processos de medição de sistemas físicos adiciona à nossa realidade um elemento aleatório fundamental. Em contraste ao elemento aleatório introduzido pela termodinâmica e pela física estatística, que tem por

3 Até o presente, ainda existem tentativas teóricas de se reestabelecer o determinismo na ciência por meio de modificações das teorias da mecânica quântica ou por meio de interpretações alternativas das teorias existentes.

4 A questão da interpretação da teoria da medição quântica é filosoficamente complexa e aborda em profundidade os conceitos apresentados neste parágrafo. Ainda que um tratamento da teoria quântica que não aborde o problema da medição quântica seja formalmente incompleto, quantitativamente existe somente uma previsão teórica a respeito da realidade em determinadas circunstâncias. Se houvesse duas diferentes, uma delas seria eliminada por um experimento físico. Mesmo assim, existem diferentes formas de interpretar os resultados experimentais e as previsões teóricas a respeito da realidade. 
base a simplificação do espaço de fases de sistemas complexos, o que provoca uma incerteza com relação ao estado do sistema. Por sua vez, as leis da mecânica quântica estabelecem que mesmo que um estado físico seja conhecido com arbitrária precisão, os resultados de processos de medição realizados sobre o sistema nesse dado estado serão, em geral, intrinsecamente aleatórios. Como consequência, as observações realizadas sobre sistemas quânticos podem ser modeladas por sistemas estocásticos. A teoria de filtros quânticos, uma generalização dos métodos matemáticos desenvolvidos para obter estimativas para o estado de um sistema clássico em geral não linear, é desenvolvida desde a década de 60 por Davies, na década de 80, Belavkin avançou no desenvolvimento da teoria e a estruturou basicamente como se encontra hoje. Na década de 90, Carmichael desenvolveu independentemente a mesma teoria, com base em argumentos heurísticos e a popularizou na comunidade física.

Com base nos fundamentos da teoria de filtros quânticos (BOUTEN; HANDEL; JAMES, 2006b), os métodos da teoria de controle estocástico, lineares e não lineares, puderam ser aplicados no desenvolvimento de sistemas de controle quânticos em malha fechada. ${ }^{5}$ Em linhas gerais, a teoria de filtros quânticos é baseada em uma generalização da teoria da probabilidade chamada teoria da probabilidade não comutativa ou teoria da probabilidade quântica, em seguida, os conceitos de esperança condicional são derivados e finalmente, uma estrutura apropriada para o cálculo estocástico com elementos não comutativos complementa o conjunto de ferramentas necessárias ao tratamento de sistemas dinâmicos quânticos envolvendo processos de medição. No cerne das teorias de probabilidade clássica e quântica, encontra-se o tratamento de eventos, que são definidos quanticamente como operadores lineares do tipo projeção ortogonal $P=P^{\dagger}=P^{2}$. Como os eventos na lógica quântica possuem uma estrutura geométrica adicional, seu comportamento difere em certos aspectos. Classicamente, é sempre possível descrever a probabilidade conjunta de dois eventos, quanticamente não, tendo em vista que as projeções ortoganais em espaços lineares nem sempre comutam.

Apresentam-se a seguir algumas definições e resultados da teoria de sistemas dinâmicos que são importantes também no contexto dos sistemas quânticos. Apresentamos um conjunto de resultados e definições tomando por base (FICHMANN; SALLUM, 2004; GELFAND; FOMIN, 1963; ANOSOV et al., 1988; ARNOLD, 1978; ARNOLD, 1997; SOTOMAYOR, 1980).

Dado um espaço de fases, $M$, um processo evolutivo ou sistema dinâmico contínuo em $M$ é descrito pelo movimento de cada ponto do espaço de fases sobre uma curva. A velocidade com que o processo evolui está definida para todos os pontos $x \in M$, ou seja, para todo $x$ está definido um vetor velocidade de fase. O conjunto de vetores

$\overline{5}$ A teoria de controle quântico apresenta questões inerentes à estrutura matemática da física quântica que não possuem similares clássicos, vide por exemplo (MABUCHI, 2014). 
velocidade associados aos pontos de $M$ é chamado campo de vetores de velocidade de fase ou simplesmente campo de vetores.

Dado um campo de vetores $v$ em $U \subset \Re^{n}$, a equação diferencial ordinária definida por $v$ é dada por:

$$
\dot{x}=\frac{d x}{d t}=v(x)
$$

A solução de uma EDO é uma curva, $\phi: I \rightarrow U$, definida em um intervalo real e tomando valores no espaço de fases $U$, cuja derivada em todos os pontos é idêntica ao vetor associado pela EDO.

$$
\dot{\phi}(t)=\frac{d \phi}{d t}(t)=v(\phi(t))
$$

Se $v$ é um campo vetorial de classe $C^{k}, k \geq 1$ em $\Re^{n}$ e existe $M>0$ tal que $M>\|v(x)\|$ para todo $x \in \Re^{n}$ então $\phi\left(x_{0}, t\right)=\phi_{x_{0}}(t)$ solução da EDO associada está definida para todo $t \in \Re$.

Similarmente, se $v$ é um campo vetorial de classe $C^{k}, k \geq 1 \mathrm{em} M$, uma variedade diferenciável compacta (por exemplo, uma esfera ou um toro), então $\phi\left(x_{0}, t\right)=\phi_{x_{0}}(t)$, solução da EDO associada, está definida para todo $t \in \Re$.

Seja $\phi: I \rightarrow U$ a solução de uma EDO no espaço de fase $U$. A imagem de $\phi$ em $U$ é chamada uma órbita da EDO em $U$ e o gráfico de $\phi$ contido em $\Re \times U$ é chamado curva integral. A decomposição de $U$ nas órbitas de uma EDO definida em $U$ é chamada retrato de fase da EDO. As órbitas são orientadas no sentido positivo de $t$.

Tipos de Órbitas: Seja $v$ um campo vetorial de classe $C^{k}, k \geq 1$ em $U$ e $\phi\left(x_{0}, t\right)=\phi_{x_{0}}(t)$ solução da EDO associada. Se $\phi_{x_{0}}: I \rightarrow U$ é solução maximal, então, verifica-se uma única:

1. $\phi_{x_{0}}$ é injetora.

2. $I=\Re$ e $\phi_{x_{0}}=x_{0}$. Em pontos singulares.

3. $I=\Re$ e $\phi_{x_{0}}$ é uma órbita periódica, ou seja, $\phi_{x_{0}}(\tau)=\phi_{x_{0}}(0)=x_{0}$ para algum $\tau>0$ chamado período da órbita.

Grupos de Difeomorfismos ${ }^{6}$ : Um grupo de difeomorfismos a um parâmetro de classe $C^{k}, k \geq 1,\left\{g^{t}\right\}$, de um domínio $U$ é uma aplicação $g: U \times \Re \rightarrow U, g(x, t)=g^{t} x$ tal que:

1. $g$ é de classe $C^{k}, k \geq 1$.

6 Pode-se consultar, por exemplo, (ANOSOV et al., 1988). 
2. Para cada $t \in \Re, g^{t}: U \rightarrow U$ é um difeomorfismo.

3. $g$ satisfaz a propriedade de grupo dada por $g^{t} \circ g^{s}=g^{t+s}$ para todo tes $\in \Re$ e $g^{0}=I d$, ou seja, $g^{0} x=x$.

A terminologia grupo de difeomorfismos quer dizer que existe um conjunto de difeomorfismos cujos elementos estão em correspondência biunívoca com os elementos de um grupo (propriedades 1 e 2). E mais, é necessário que a correspondência respeite a operação do grupo (propriedade 3). O grupo, $G$, representa o espaço em está definido o tempo, a variável independente que evolui.

Sistema Dinâmico: Dado um grupo, $(G,+)$, e um espaço de fases, $S$, um sistema dinâmico é dado por uma aplicação $\Phi: G \times S \rightarrow S$ em que, escrevendo-se $\Phi^{t}(x)=\Phi(t, x)$, o mapa $\Phi^{t}: S \rightarrow S$ satisfaz:

1. $\Phi^{0}: S \rightarrow S$ é a apliacação identidade e 0 é o elemento neutro do grupo

2. $\Phi^{t} \circ \Phi^{s}=\Phi^{t+s}$

Com essa definição genérica de sistema dinâmico estamos englobando pelo menos dois tipos de sistemas. Os sistemas discretos, quando $G$ é o grupo $(Z,+)$ dos números inteiros, e os sistemas contínuos quando $G$ é o grupo $(\Re,+)$ dos números reais ${ }^{7}$. Quando o sistema dinâmico é contínuo, podemos exigir que o mapa $(t, x) \mapsto \Phi^{t} x$ seja um difeomorfismo. Nesse caso tem-se um sistema dinâmico diferenciável que é equivalente a um grupo de difeomorfismos. Quando a aplicação $(t, x) \mapsto \Phi^{t} x$ é apenas um homeomorfismo tem-se um sistema dinâmico topológico. Sistemas dinâmicos contínuos são também chamados fluxos.

Até o presente momento estamos considerando apenas sistemas com mudanças de estado determinísticas, ou seja, completamente determinadas pelos campos de vetores estudados. Dado um ponto no espaço de fases, pode-se destacar a órbita da EDO que passa por este ponto. Como consequência, fica completamente determinado o processo de evolução do ponto pela ação do campo de vetores. Tanto os estados passados quanto os estados futuros ficam determinados, uma vez que a evolução de um ponto qualquer do espaço de fases é restrita à sua própria órbita. Elementos de aleatoriedade podem entrar na teoria de sistemas dinâmicos determinísticos apenas pela análise da evolução temporal de uma distribuição de probabilidades no espaço de fases.

No caso de sistemas dinâmicos estocásticos, existe presente um elemento aleatório na própria dinâmica de evolução do sistema. Como consequência, mesmo que esteja

7 No caso de grupos finitos, os sistemas dinâmicos definidos são capazes de representar autômatos finitos, estruturas estudadas em lógica e teoria da computação e que estão relacionadas à definição da máquina de Turing como dispositivo fundamental da computação clássica. 
determinado o estado $x_{0} \in U$ do sistema em um instante de tempo $t_{0}$, não é possível afirmar qual será o estado $x_{1}$, após a evolução do sistema pelo período $t_{1}-t_{0}$. Em geral, os sistemas dinâmicos estocásticos estabelecem a evolução de distribuições de probabilidade sobre os espaço de fases. Existe uma diferença sutil entre a evolução determinística e a evolução estocástica de uma distribuição de probabilidades sobre um espaço de fases. Essa diferença será essencial à compreensão do operador densidade, que caracteriza o estado de um sistema quântico, e da relação entre o estado de um sistema quântico e o resultado de um processo de medição a ele relacionado.

\subsection{Controle de Sistemas Clássicos}

Esta seção tem por objetivo fixar a terminologia padrão utilizada no campo da teoria matemática de controle. Possui natureza apenas introdutória, uma vez que os aspectos mais avançados serão tratados em seções posteriores, quando houver necessidade. O Anexo B apresenta definições e resultados importantes da área de sistemas dinâmicos.

Dado um sistema dinâmico qualquer, assume-se que seu estado físico possa ser representado por um ponto bem definido em um conjunto conveniente, $B$, geralmente um espaço linear normado ${ }^{8}$, (GELFAND; FOMIN, 1963). Esse conjunto é denominado espaço de estados. A evolução temporal desse sistema físico é representada por uma curva bem definida no espaço $B$, (LANCZOS, 1949). Cada curva em $B$ é denominada um processo dinâmico.

Alguns aspectos devem ser assumidos para que o modelo matemático esteja bem definido. Um deles é que exista uma correspondência biunívoca entre estados físicos e pontos de $B$. Assim, a cada estado físico deve corresponder um ponto do espaço matemático subjacente.

A evolução temporal de um sistema físico determinístico é descrita matematicamente através de equações diferenciais ordinárias cuja solução fornece curvas, ou processos dinâmicos bem definidos em B, (FICHMANN; SALLUM, 2004). Nesse ponto, pode-se fazer distinção entre dinâmica livre e dinâmica forçada. Na dinâmica livre, o sistema está sujeito apenas a uma interação com um campo de energia externo autônomo, ou seja, independente do tempo. Já na dinâmica forçada, o campo externo é modificado em função do tempo. E tem-se, no caso de espaço de estados n-dimensional:

$$
\frac{d x_{i}}{d t}=f_{i}\left(x_{1}, \ldots, x_{n}, u_{1}, \ldots, u_{k}\right)
$$

8 Os sistemas dinâmicos podem ser definidos de forma mais geral em variedades diferenciáveis. Em particular podem ser considerados sistemas dinâmicos em grupos de Lie. A dinâmica de sistemas quânticos pode ser estudada no grupo $U(n)$ ou $S U(n)$. 
em que $x_{i}$ são coordenadas no espaço de estados e os coeficientes $u_{j}(t)$, que variam no tempo, são as variáveis ou parâmetros de controle. Os $u_{j}$ podem ser identificados com as parcelas não autônomas do campo de energia externo.

A observação do sistema é também um processo dinâmico e pode ser modelada em um espaço m-dimensional por:

$$
y_{i}=g_{i}\left(x_{1}, \ldots, x_{n}, u_{1}, \ldots, u_{k}\right)
$$

Observar um sistema físico é obter informação a respeito de seu estado físico. A observabilidade de um modelo matemático de um sistema físico está relacionada com a possibilidade de se obter informação total ou parcial a respeito de seu estado físico a partir de um conjunto de variáveis medidas.

Controlar um sistema físico é fazê-lo passar de um estado físico inicial para um estado físico final. A controlabilidade de um sistema está relacionada à possibilidade de se encontrar um processo dinâmico capaz de evoluir um sistema físico de um estado inicial qualquer para um estado físico final qualquer, chamado estado objetivo.

Em sua forma vetorial geral, as equações podem ser escritas como:

$$
\begin{aligned}
\dot{x}(t) & =F(x(t), u(t)) \\
y(t) & =G(x(t), u(t))
\end{aligned}
$$

O sistema acima descreve a ação de um campo de vetores não linear no espaço de estados. A solução da equação diferencial associada define um fluxo no espaço de estados. Esse fluxo é parametrizado pelas variáveis de controle $u(t) \in \Re^{p}$. Este fluxo nada mais é que o conjunto de órbitas, ou processos dinâmicos, que têm como ponto de partida cada $x_{0} \in B$. O observador tem acesso aos resultados das medições realizadas continuamente e representadas pelo vetor $y(t)$. O processo de medição é modelado pela equação algébrica $G$, que depende tanto do estado dinâmico do sistema, $x(t)$, quanto das variáveis ou parâmetros de controle, $u(t)$. Toda informação disponível ao observador a respeito do estado dinâmico do sistema está contida no vetor de medições, $y(t)$.

Se, dados dois pontos em $B$, existir um processo dinâmico que una estes pontos, diz-se que esses dois pontos são alcancáveis entre si. Se o sistema físico for reversível, esta propriedade define uma relação de equivalência em $B$. Nessas condições, se o conjunto de estados alcançáveis for denso no conjunto de estados objetivo, o controle pode ser estabelecido no espaço com precisão arbitrária e o sistema é dito controlável.

Se, a partir do vetor de medições $y(t)$, o observador for capaz de reconstituir o estado dinâmico do sistema, $x(t)$, o sistema é dito observável.

A nomenclatura padrão na engenharia de sistemas e controle utiliza os termos entrada e saida para denominar, respectivamente, os vetores $u(t)$ e $y(t)$, ao passo que o 
sistema dinâmico a ser controlado é denominado planta. As estratégias estudadas nas teorias de controle por realimentação têm por objetivo escolher o melhor sinal de entrada a ser injetado na planta, a partir do sinal medido em sua saída, de acordo com o estado objetivo a ser alcançado. Para que uma estratégia de controle por realimentação tenha pleno sucesso, é necessário que o sistema seja observável e controlável.

Tanto a controlabilidade quanto a observabilidade são propriedades intrínsecas da planta, ou seja, do sistema dinâmico definido pelo campo de vetores $F(x, u)$. Os sistemas de controle do tipo afim tornam explícitos os campos de vetores em ação no sistema dinâmico:

$$
\begin{aligned}
& \dot{x}(t)=F_{0}(x(t))+u_{1}(t) F_{1}(x(t))+u_{2}(t) F_{2}(x(t))+\ldots+u_{n}(t) F_{n}(x(t)) \\
& y(t)=G\left(x(t), u_{1}(t), \ldots, u_{n}(t)\right)
\end{aligned}
$$

Resultados importantes e gerais da teoria de controle geométrico, que tem fundamento em métodos da geometria diferencial, relacionam a controlabilidade e a observabilidade de um sistema de controle afim a propriedades da família de campos de vetores $\left\{F_{i}\right\}$. Maiores detalhes podem ser buscados em (JURDJEVIC, 1997).

O conjunto de campos de vetores $F(B)$ definidos em um espaço $B$ é uma álgebra de Lie com operação de multiplicação definida pelo parenteses de Lie, dado por $[X, Y]=$ $Y \circ X-X \circ Y$, que define o comutador entre dois campos de vetores $X, Y \in F(B)$. A subalgebra de Lie gerada por uma família de campos de vetores $\left\{F_{i}\right\}$ é denotada por $\operatorname{Lie}\left(F_{i}\right)$. E $\operatorname{Lie}_{x}(F)$ denota o conjunto $V(x)$ de vetores tangentes a $B$ em $x \in B$, tais que $V \in F$.

Localmente, é possível afirmar que se a família de campos de vetores $F_{i}$ é tal que $\operatorname{Lie}_{x}\left(F_{i}\right)=T_{x} B$ para $x \in B$, então, a órbita de $F_{i}$ por $x$ é um conjunto aberto em $B$. A órbita de uma família de campos de vetores $F_{i}$ por um ponto $x_{0} \in B$ é dada por $E(x)=\left\{x \in B: x=\phi\left(x_{0}\right)\right\}$ e $\phi$ é um elemento do grupo, ou pseudogrupo, de difeomorfismos, ou difeomorfismos locais, gerado por $X \in\left\{F_{i}\right\}$.

Em termos globais, pelo teorema da órbita, a órbita de um ponto $x_{0} \in B$ por uma família de campos de vetores $\left\{F_{i}\right\}$ é uma subvariedade $U \subseteq B$. O famoso teorema de Frobenius relaciona a família de campos de vetores ao espaço tangente $T_{x} U$.

Uma família de campos de vetores $F$ é chamada Lie determinada se o espaço tangente a cada ponto $x$ em sua órbita $E(x), T_{x} U$, concide com a álgebra de Lie gerada por $F$ em $x, \operatorname{Lie}_{x}(F)$. Todos os sistemas de controle análiticos são Lie determinados. O conjunto de pontos acessíveis em $B$ pela órbita da família de campos de vetores $F$ em $x \in B$ é denotado por $\mathcal{A}_{F}(x)$.

Seja $F$ uma família de campos de vetores do tipo Lie determinada, o saturado de Lie de $\mathrm{F}$, denotado por $\mathcal{L} \mathcal{S}(F)$ é o maior subconjunto $\hat{F}$ de $\operatorname{Lie}(F)$ tal que o fecho topológico de $\mathcal{A}_{\hat{F}}(x)$ é igual ao fecho topológico de $\mathcal{A}_{F}(x)$. 
O sistema de controle definido por uma família de campos de vetores $\left\{F_{i}\right\}$ é controlável no espaço $B$ se, e somente se, $F$ é uma família de campos de vetores do tipo Lie determinada, o saturado de Lie é igual a $\operatorname{Lie}\{F\}$ e $\operatorname{Lie}_{x}(F)=T_{x} B$ para todo $x \in B$.

É fácil perceber que a solução do problema da controlabilidade para sistemas não lineares não é trivial. Importante extrair dessa digressão que a álgebra de Lie dos campos de vetores associados ao sistema de controle é uma estrutura essencial à solução do problema. Outra questão de importância fundamental é que a controlabilidade depende de propriedades topológicas das órbitas geradas pelos campos de vetores no espaço subjacente. De acordo com (JURDJEVIC, 1997), o conjunto representado pelo fecho com conjunto acessível é recorrente na teoria geométrica de controle. No entanto, o comportamento desse conjunto somente pode ser determinado em termos gerais para sistemas de controle particulares, para os quais os campos de vetores e o espaço de estados possuem estrutura adicional.

A linearização de sistemas de controle leva à consideração de sistemas de controle lineares e bilineares conforme definições a seguir. Tais sistemas são importantes por si mesmos, uma vez que será mostrado na sequência que uma classe considerável de sistemas de controle quânticos é do tipo bilinear. Além disso, a linearização é importante para estudar propriedades locais de sistemas de controle não lineares complexos.

O sistema de controle linear é definido por:

$$
\begin{aligned}
& \dot{x}(t)=A x(t)+B u(t)) \\
& y(t)=C x(t)
\end{aligned}
$$

E um sistema de controle bilinear por:

$$
\begin{aligned}
\dot{x}(t) & =\left(A_{0}+A_{i} u_{i}(t)\right) x(t) \\
y(t) & =C x(t)
\end{aligned}
$$

Para o caso de sistemas lineares, é importante perceber como o estudo e a aplicação ferramentas relacionadas às algebras de Lie pode nos conduzir à conclusão de que se trata de um caminho para a generalização do critério de Kalman para definir a controlabilidade de sistemas lineares. O exemplo a seguir é extraído de (JURDJEVIC, 1997).

Seja $X$ um campo de vetores linear em $\Re^{n}$ dado por $x \mapsto A x$ e $Y$ um campo de vetores constante dado por $b \in \Re^{n}$. Lie $(\{X, Y\})$ é equivalente ao espaço vetorial gerado por $X$ e por $A d^{(k)} X(Y)$, em que a operação anterior é definida a partir de $\operatorname{AdX}(Y)=$ $[X, Y]$. Então, $A d^{(k)} X(Y)=A^{k} b$. O teorema de Cayley-Hamilton estabelece que $A^{n}$ é linearmente dependente de $\left\{A^{0}, A^{1}, \ldots, A^{n-1}\right\}$. Portanto, $A d^{(k)} X(Y)$ é uma álgebra de Lie de dimensão finita e $\operatorname{Lie}_{x}(\{X, Y\})$ é equivalente ao subespaço vetorial gerado por $\left\{A x, b, A b, A^{2} b, \ldots, A^{n-1} b\right\}$. Assim, na origem, $\operatorname{Lie}_{x}(\{X, Y\})$ é o subespaço vetorial gerado por $\left\{b, A b, A^{2} b, \ldots, A^{n-1} b\right\}$. 
Outros aspectos importantes na modelagem de sistemas físicos são as incertezas relacionadas à medição e à robustez da representação matemática. Um primeiro aspecto fundamental é a existência de ruído intrínseco em qualquer aparato físico de medição. Como consequência quaisquer estimativas associadas ao estado do sistema serão vinculadas a uma incerteza. A simples existência desse ruído já introduz aspectos estocásticos no modelo matemático. Assim, como não se possui informação completa a respeito do estado físico de um sistema, não é possível determinar com certeza a exata curva integral percorrida pelo sistema ao longo de sua evolução. E a incerteza pode crescer com o tempo de evolução. Já a robustez da representação matemática está relacionada com a correspondência entre a evolução do sistema físico e a equação diferencial que o representa matematicamente. Em geral, a equação diferencial é uma simplificação de uma equação mais geral, ou então a equação depende de parâmetros físicos que devem ser medidos experimentalmente. A inexistência de sistemas físicos isolados também faz com que a modelagem matemática completa seja complicada, pois é praticamente impossível incorporar todos os aspectos da realidade física em uma equação diferencial. Esses pormenores introduzem incerteza na própria lei de evolução do modelo matemático.

\subsection{Dinâmica de Sistemas Quânticos}

Encontram-se a seguir os postulados da mecânica quântica de acordo com sua apresentação usual em monografias de física, por exemplo, em (SAKURAI, 1994; BASDEVANT; DALIBARD, 2005; BOHM, 1994; COHEN-TANNOUDJI; DIU; LALOë, 1977; GRIFFITHS, 2005). Nesta seção nos limitaremos a apresentar o suficiente da teoria para compreender os principais aspectos dos modelos matemáticos de sistemas quânticos discretos em dimensão finita. ${ }^{9}$ A apresentação na forma de postulados facilita o entendimento dos princípios fundamentais, ao mesmo tempo em que mostra quais são os elementos básicos da teoria. Um amplo panorama dos aspectos fundamentais do tratamento matemático formal de sistemas quânticos fechados pode ser encontrado em (LISBOA, 2015).

Para descrever a dinâmica de um sistema, é necessário definir matematicamente um conjunto em que cada elemento seja capaz de representar um estado físico real de um sistema. Também é necessário que, dentro do escopo dos fenômenos físicos modelados, que para cada estado físico real corresponda um elemento deste conjunto, que é denominado espaço de estados. Em seguida, é necessário descrever matematicamente, no espaço de estados, como se dá a evolução temporal do sistema.

9 Os axiomas podem ser extendidos para espaços de Hilbert de dimensão infinita utilizando os métodos usuais da análise funcional. Foge ao escopo deste trabalho apresentar a teoria quântica neste nível de generalidade. Ao longo do texto, na medida da necessidade, os aspectos relacionados à análise funcional serão abordados pontualmente. 


\subsubsection{Formulação matemática em espaço de estados}

Postulado 1: Espaço de estados - Associado a cada sistema físico isolado, há um espaço vetorial complexo com produto interno, ou seja, um espaço de Hilbert $\mathcal{E}$ denominado espaço de estados do sistema. A cada instante de tempo, $t_{0}$, o sistema físico é completamente descrito por seu vetor de estado de norma unitária, $\left|\psi\left(t_{0}\right)\right\rangle \in \mathcal{E}^{10}$.

O espaço de Hilbert é um espaço vetorial sobre o corpo dos números complexos, portanto, linear. O produto escalar no espaço de Hilbert é hermitiano:

1. $\langle\phi \mid \psi\rangle=\langle\psi \mid \phi\rangle^{*}$

2. Se $|\psi\rangle=c_{1}\left|\psi_{1}\right\rangle+c_{2}\left|\psi_{2}\right\rangle$ então $\langle\phi \mid \psi\rangle=c_{1}\left\langle\phi \mid \psi_{1}\right\rangle+c_{2}\left\langle\phi \mid \psi_{2}\right\rangle$

3. $\langle\psi \mid \psi\rangle \geq 0$

4. $\langle\psi \mid \psi\rangle=0$ se e somente se $|\psi\rangle=0$

A norma de um vetor no espaço de Hilbert é induzida pelo produto escalar e é dada por $\||\psi\rangle \|=\sqrt{\langle\psi \mid \psi\rangle}$. Dois vetores que diferem entre si apenas por uma constante multiplicativa não nula representam um mesmo estado quântico. Assim, no espaço de Hilbert, um estado quântico é melhor visualizado geometricamente como um raio passando pela origem. ${ }^{11}$

Postulado 2: Descrição de quantidades físicas observáveis - Toda quantidade física $\mathcal{A}$ capaz de ser medida em um sistema físico é associada a um operador linear hermitiano $A: \mathcal{E} \rightarrow \mathcal{E}$, este operador é denominado um observável do sistema.

Postulado 3: Resultados possíveis em processos de medição - Os únicos resultados possíveis em um processo de medição de uma determinada quantidade física, $\mathcal{A}$, em um sistema quântico, são os autovalores do observável associado $A$.

Postulado 4: Natureza probabilística dos processos de medição - No processo de medição da quantidade física $\mathcal{A}$, sejam $\left\{a_{i}\right\}$ o conjunto de autovalores não degenerados de $A$ e $\left\{\left|a_{i}\right\rangle\right\}$ seus respectivos autovetores. A probabilidade de se obter $a_{0}$ como resultado do processo de medição do observável $A$ no sistema que se encontra no estado $|\psi\rangle$ é dada por:

$$
P\left(a_{0}\right)=\left|\left\langle a_{0} \mid \psi\right\rangle\right|^{2}
$$

10 O produto interno será denotado utilizando a notação de Dirac, $\langle\phi \mid \psi\rangle$, na qual o primeiro termo é denominado "bra" e o segundo termo é denominado "quet".

11 A necessidade de deixar bem definidas as probabilidades resultantes de um processo de medição, a ser definido na sequência, exige que o vetor tenha uma norma unitária, no entanto a multiplicação por qualquer escalar complexo de norma unitária mantém inalterado o estado físico. São também excluídos fisicamente os "vetores" que não possuem norma finita. 
Postulado 5: Efeito do processo de medição no sistema - Caso o processo de medição do observável $A$ no sistema em estado $|\psi\rangle$ tenha resultado em $a_{0}$, o estado, $\left|\psi_{m}\right\rangle$, do sistema imediatamente após a medição realizada é dado por:

$$
\left|\psi_{m}\right\rangle=\frac{\Lambda_{0}|\psi\rangle}{\sqrt{\left\langle\psi\left|\Lambda_{0}\right| \psi\right\rangle}}
$$

em que $\Lambda_{0}$ é operador de projeção no subespaço vetorial gerado pelo autovetor $\left|a_{0}\right\rangle$.

Postulado 6: Evolução temporal do sistema - A evolução temporal do sistema é governada por uma equação diferencial denominada equação de Schrödinger:

$$
i \hbar \frac{d}{d t}|\psi\rangle=H(t)|\psi\rangle
$$

em que $H(t)$, denominado operador Hamiltoniano, é o observável associado à energia total do sistema.

De forma equivalente, o estado do sistema no tempo $t$ pode ser calculado a partir do estado do sistema no instante $t_{0}$ utilizando o operador unitário de evolução, $U\left(t, t_{0}\right)$ associado à equação de Schrödinger, utilizando:

$$
|\psi(t)\rangle=U\left(t, t_{0}\right)|\psi(t)\rangle
$$

O operador de evolução, também denominado propagador, é solução da equação de Schrödinger para operadores:

$$
i \hbar \dot{U}(t)=H(t) U(t)
$$

No livro Quantum Computation and Quantum Information (NIELSEN; CHUANG, 2000), os autores acrescentam outro postulado, associado à descrição de sistema quânticos compostos de várias partes, e dão maior ênfase aos mecanismos de medição das propriedades de um sistema quântico. A numeração desse e de outros postulados que seguirão continuará da numeração iniciada acima para facilitar referências futuras.

Postulado \%: Descrição de sistemas compostos - O espaço de estados de um sistema quântico composto é o produto tensorial dos espaços de estados dos sistemas quânticos componentes. Se o sistema $E_{a}$ estiver no estado $\left|\psi_{a}\right\rangle \in \mathcal{E}_{a}$ e o sistema $E_{b}$ estiver no estado $\left|\psi_{b}\right\rangle \in \mathcal{E}_{b}$, então, o sistema quântico composto encontrar-se-á no estado $\left|\psi_{a}\right\rangle \otimes\left|\psi_{b}\right\rangle$. Como o espaço de estado dado pelo produto tensorial dos espaçoes de estado componentes é também um espaço linear, então, o estado dado por $|\gamma\rangle=\left|\psi_{a}\right\rangle \otimes\left|\psi_{b}\right\rangle+\left|\phi_{a}\right\rangle \otimes\left|\phi_{b}\right\rangle$ também representa um estado físico real. Nem sempre existem estados $|\alpha\rangle \in \mathcal{E}_{a}$ e $|\beta\rangle \in \mathcal{E}_{b}$ tais 
que o estado do sistema composto seja dado por $|\alpha\rangle \otimes|\beta\rangle$. Esses estados do tipo $|\gamma\rangle$, com essa propriedade, são ditos emaranhados.

\subsubsection{Formulação matemática por meio de operadores densidade}

Os postulados apresentados a seguir possuem alguma redundância com relação aos apresentados até o momento. No entanto, no tratamento de sistemas quânticos que apresentam incerteza a respeito de seu estado, a descrição por meio de vetores, como realizada até o momento, é insuficiente.

Postulado 8: Abrangência descritiva do operador densidade - Encontra-se associado a todo sistema quântico isolado um espaço vetorial complexo com produto interno (Um espaço de Hilbert) denominado espaço de estados. O sistema físico é completamente descrito por meio de um operador densidade $\rho$, que consiste em um operador linear positivo semidefinido com traço unitário. Se o estado de um sistema puder ser particionado de tal forma que cada uma das partes esteja no estado $\rho_{i}$ com probabilidade $p_{i}$, então o estado do sistema completo é dado por $\sum_{i} p_{i} \rho_{i}$.

Postulado 9: Evolução temporal - A evolução temporal de um sistema quântico fechado, isto é, de um sistema quântico completamente isolado do ambiente externo, é descrita por uma transformação unitária. Assim, o estado $\rho_{i}$ de um sistema no instante $t_{i}$ relaciona-se ao estado $\rho_{f}$ do sistema no instante $t_{f}$ por meio de uma transformação unitária $U$ que depende apenas dos instantes inicial e final, $t_{i}$ e $t_{f}$ :

$$
\rho_{f}=U \rho_{i} U^{\dagger}
$$

A transformação unitária é obtida por meio da equação de Schrödinger para operadores descrita no postulado 6 acima.

Postulado 10: Processos de medição - Os processos de medição quânticos são descritos, utilizando a representação matemática por meio de operadores densidade, através de uma família de operadores $\left\{M_{m}\right\}$. Trata-se de operadores lineares no espaço de estados do sistema. O índice $m$ se refere aos diferentes resultados possíveis que podem ocorrer no experimento de medição. Se um sistema quântico estiver no estado $\rho$ antes da medição, então, a probabilidade de ocorrer o resultado $m$ é dada por:

$$
p_{m}=\operatorname{Tr}\left(M_{m}^{\dagger} M_{m} \rho\right)
$$

E o estado do sistema imediatamente após o experimento de medição que tenha 
resultado em $m$ será dado por $^{12}$ :

$$
\rho_{m}=\frac{\left.M_{m} \rho M_{m}^{\dagger}\right)}{\operatorname{Tr}\left(M_{m}^{\dagger} M_{m} \rho\right)}
$$

Os operadores de medição devem satisfazer uma equação de completude no espaço de Hilbert, ou seja, uma família de operadores de medição está relacionada a uma partição da unidade da seguinte forma:

$$
\sum_{m} M_{m}^{\dagger} M_{m}=I
$$

Postulado 11: Descrição de sistemas compostos - O espaço de estados de um sistema quântico composto é dado pelo produto tensorial entre os espaços de estados de cada uma das partes. Ainda, se tivermos um sistema composto por $n$ partes e cada uma delas estiver no estado $\rho_{i}$, o estado do sistema composto será dado por:

$$
\rho_{\text {total }}=\rho_{1} \otimes \rho_{2} \otimes \ldots \otimes \rho_{n}
$$

\subsubsection{Princípio da Incerteza de Heisenberg e Processos de Medição}

A formulação matricial da mecânica quântica faz com o produto de operadores seja não comutativo, pois, dados dois operadores $A$ e $B$, sejam eles representados por matrizes hermitianas ou unitárias, tem-se que, em geral, $A B-B A \neq 0$. Este fato, leva à definição do chamado comutador $[A, B]=A B-B A$. Caso o comutador de dois observáveis seja nulo, os observáveis são ditos compatíveis. Caso contrário, os observáveis são chamados incompatíveis.

Princípio da Incerteza - Dados dois observáveis não compatíveis $A$ e $B$, o desvio padrão nas medições de $A$ e $B$, representado por $\Delta(A)$ e $\Delta(B)$ respectivamente, é tal que, para cada estado $|\psi\rangle$ :

$$
\Delta(A) \Delta(B) \geq\langle\psi|[A, B]| \psi\rangle
$$

O princípio de Heisenberg nos informa que, se realizarmos um grande número de medições, ora do observável $A$ ora do observável $B$, em um sistema físico preparado no mesmo estado $|\psi\rangle$, estatisticamente comprovaremos que existe um limite bem definido para o grau de certeza que um observador pode ter, no caso de medições de observáveis não compatíveis. Esse princípio contrasta com a física clássica, pela qual, em teoria, não existe limite para este grau de certeza.

Os postulados 5 e 10, comumente conhecidos por "Colapso do pacote de onda", guardam uma relação próxima com o princípio da incerteza.

12 É interessante relembrar que o traço possui uma propriedade cíclica, ou seja, $\operatorname{Tr}(A B C)=$ $\operatorname{Tr}(C A B)=\operatorname{Tr}(B C A)$. 


\subsubsection{Medição de Sistemas Quânticos}

Os experimentos ideiais de medição em sistemas quânticos são descritos pelos postulados 5, para estados puros, e postulado 10, para estados descritos por operadores densidade. Os postulados e o tipo de medição que descrevem são devidos a von Neumann e Lüders e recebem muitas vezes esse nome na literatura. As medições desse tipo são também denominadas medições ortogonais.

As formas generalizadas de realização de medições em sistemas quânticos serão descritas no capítulo 4.

\subsection{Controle de Sistemas Quânticos}

O modelo matemático de um sistema dinâmico quântico é composto em sua base por um espaço de Hilbert. Sobre essa estrutura, são definidas duas entidades fundamentais: o estado do sistema e seus observáveis. Quanto ao estado do sistema, não há nenhuma peculiaridade adicional quando se compara a estrutura da definição quântica à da definição clássica. Em ambos os casos, existe um conjunto de elementos matemáticos e os estados são selecionados desse conjunto subjacente. No caso de sistemas quânticos, é conveniente escolher os estados entre os elementos do espaço de Hilbert que possuem norma unitária, em função da interpretação probabilística da teoria e da necessidade de atribuir aos estados uma norma finita. De forma equivalente, os estados podem ser definidos como os raios do espaço de Hilbert, ou seja, como os subespaços vetoriais unidimensionais gerados por elementos distintos do espaço. Uma das consequências da interpretação probabilística da teoria quântica é a multiplicação de um estado por um escalar de norma unitária não é fisicamente detectável, assim, existe uma redundância na definição dos estados quânticos nos espaços de Hilbert.Com relação aos observáveis, no entanto, as teorias clássica e quântica diferem bastante.

De acordo a interpretação física usualmente realizada no contexto clássico, a definição de um observável é totalmente dispensável. Assume-se que o estado de um sistema pode ser conhecido de forma ampla e irrestrita. Assim, no caso de um sistema mecânico, todas as posições e momentos lineares podem ser conhecidos ao mesmo tempo, permitindo a obtenção de todas as grandezas físicas de interesse, por exemplo a energia total do sistema. Existe, contudo, no contexto clássico, uma teoria de medição que busca a compreensão e a manipulação de sistemas a respeitos dos quais não se dispões de informação completa sobre seu estado. Um estado generalizado em um sistema estocástico é representado por uma distribuição de probabilidade no espaço de estados. Tanto os processos de medição quanto os processos dinâmicos aos quais o sistema está sujeito modificam a distribuição de probabilidade que representa o estado do sistema estocástico. Os processos de medição clássicos modificam o estado generalizado na medida em que introduzem mais 
informação a respeito do sistema. Classicamente também não se exclui a possibilidade de os processos de medição introduzirem modificações na dinâmica do sistema. Embora esta não seja uma característica essencial dos processos de medição clássicos.

Quanticamente a observação de um sistema não ocorre de forma trivial e o modo como a observação é definida na teoria quântica introduz um conjunto de aspectos peculiares à teoria. Tradicionalmente, os observáveis quânticos são definidos como operadores lineares auto adjuntos no espaço de Hilbert subjacente ao sistema. De acordo os postulados de medição de von Neumann, as grandezas físicas sob medição estão associadas ao espectro do operador observável e o processo de medição é descrito apenas em função de seu resultado. É importante notar que os resultados possíveis do processo de medição são os elementos do espectro do observável e que o processo de medição modifica o estado do sistema, que passará a corresponder ao vetor próprio do observável, correspondente ao valor próprio obtido pela medição. Assim, a teoria quântica de medição estabelece que o processo de medição modifica necessariamente e de forma irreversível o estado do sistema quântico. Quanticamente, então, um observável está relacionado a uma possibilidade de observação. Outra particularidade dos sistemas quânticos está relacionada à definição de observáveis. Sabe-se que em geral os operadores lineares auto adjuntos não comutam entre si. Como consequência, propriedades físicas relacionadas a observáveis representados por operadores lineares auto adjuntos que não comutam entre si não podem ser conhecidas ao mesmo tempo. A teoria quântica estabelece um limite para a quantidade de informação que pode ser obtida de um sistema físico. Esse limite é conhecido como o princípio da incerteza de Heisenberg que, em sua forma mais usual, estabelece que a posição e o momento linear de um sistema quântico somente podem ser conhecidas até o limite que o produto das incertezas relacionadas à posição e ao momento são iguais a $\hbar / 2 \pi$. É importante notar que, até o momento, ainda não foram introduzidas incertezas com relação ao estado quântico.

A teoria de medição quântica estabelece diferentes classes de processos de medição. O processo descrito acima é do tipo discreto, extrai a máxima quantidade de informação disponível no estado quântico e o modifica em função da informação obtida. Há também processos de medição contínuos, para os quais se pode estabelecer a intensidade do processo de medição, ou seja, sua taxa de extração de informação. É por meio da utilização de técnicas de medição contínua de observáveis que a teoria clássica de controle de sistemas dinâmicos pode ser aplicada no contexto quântico. De acordo com o teorema espectral, tanto no contexto da álgebra linear em dimensão finita quanto do contexto da análise funcional em dimensão infinita, a cada operador linear auto adjunto está associado um espectro, dado por um subconjunto de $\Re$ e uma família espectral, dada por um conjunto de projeções ortogonais associadas aos vetores próprios ${ }^{13}$ do operador.

$\overline{13}$ No âmbito da análise funcional, nem sempre é possível utilizar corretamente essa terminologia. Isso ocorre porque em um espaço de Hilbert, aos elementos do espectro associados ao espectro contínuo, 
A teoria quântica introduz os operadores lineares relacionados aos observáveis como elementos fundamentais. Em virtude disso, a caracterização matemática dos operadores lineares auto adjuntos ganha particular relevância. De particular importância é o fato de as grandezas físicas vinculadas a um determinado sistema físico estarem relacionadas ao espectro de operadores lineares auto adjuntos. É dessa perspectiva que o controle de sistemas quânticos ganha contornos próprios. Controlar um sistema, em termos gerais, é modificar um subconjunto de seus observáveis. E, de acordo com a representação matemática apresentada acima, tal fato representa a manipulação do espectro de operadores lineares auto adjuntos. Tanto as modificações no estado introduzidas pelos processos de medição quanto a dinâmica própria do sistema devem ser incorporadas ao modelo matemático de um sistema dinâmico quântico para que se possa estudar sistemas quânticos controlados por realimentação ${ }^{14}$.

É também uma característica essencial dos sistemas dinâmicos quânticos que a definição do conjunto relevante de observáveis de um sistema estabelece certos sistemas de coordenadas como preferenciais no espaço de estados, ou seja, no espaço de Hilbert. Esses sistemas de coordenadas estão associados às famílias espectrais dos operadores lineares auto adjuntos ${ }^{15}$. As famílias espectrais definem as direções preferenciais no espaço para as quais o estado do sistema pode ser modificado, no caso de medições discretas de von Neumann. No caso de medições contínuas, a família espectral define direções que são pontos fixos ou pontos de equilíbrio dos processos de medição, ou seja, à medida em que a informação é extraída de um estado, sua orientação vai se alinhando a uma das direções preferenciais. No entanto, o processo de medição é essencialmente aleatório. Assim, dado que o estado inicial do sistema não seja um autovetor do observável, o resultado do processo de medição pode ser qualquer um dos elementos do espectro do operador linear auto adjunto, desde que o estado tenha projeção não nula sobre a direção principal associada ao autovalor ${ }^{16}$.

Além do controle de sistemas quânticos por realimentação, existem outras técnicas de controle, por exemplo, o controle em malha aberta, que também possui similar clássico como o controle por realimentação, e o chamado controle coerente, não existente fora do

não corresponde um autovetor dado por um elemento do espaço. Fisicamente se utiliza a função delta de Dirac para representar esses autovetores. Essa linguagem comum na física pode ser colocada em uma sólida base matemática, utilizando-se a teoria Espaços de Hilbert Equipados ou Rigged Hilbert Spaces que adiciona às funções convencionais, que são os elementos do espaço de Hilbert, as distribuições, entre as quais se encontra a "função" delta de Dirac, que não é propriamente uma função, mas sim uma distribuição.

14 A compreensão dos mecanismos e dos modelos matemáticos relacionados ao controle de sistemas quânticos por realimentação é também um dos objetivos gerais deste trabalho

15 Essas afirmações estão relacionadas aos espaços de Hilbert de dimensão finita. Nos espaços de dimensão infinita somente fazem sentido quando aplicadas aos operadores lineares auto adjuntos que não possuem espectro contínuo. Nesses casos, o conjunto de autovetores é ortogonal e define uma base no espaço vetorial e, portanto, um sistema de coordenadas.

16 A teoria permite o cálculo das probabilidades, que são proporcionais à projeção do estado sobre as direções definidas pelos autovetores 
contexto quântico. No controle coerente, as trocas de energia associadas tanto ao fluxo de informação quanto à cadeia de comando das variáveis dinâmicas são realizadas atraveś de processos quânticos. Como consequência, os efeitos relacionadas aos processos de medição são contornados.

Nos termos da metodologia científica, uma determinada coisa tem sua existência estabelecida apenas a partir da observação criteriosa de seu comportamento ${ }^{17}$.

Para o caso de sistemas quânticos de dimensão finita cuja dinâmica não está vinculada a processo de medição discretos ou contínuos a estrutura matemática de seus sistemas de controle é um caso particular da equação 1.3 acima. No caso dos sistemas quânticos, a dinâmica é descrita pela equação de Schrödinger. Seja $|\psi\rangle$ o estado de uma sistema quântico, representado por um vetor coluna em um espaço vetorial de dimensão finita. Para os sistemas de controle quânticos, a energia do sistema depende de algumas funções temporais que desempenham o papel de parâmetros de controle. Nos exemplos mais comuns descritos na literatura, essas funções modelam campos eletromagnéticos variáveis no tempo que podem ser considerados constantes no espaço para efeitos práticos. Em consequência, o Hamiltoniano do sistema quântico é dependente do tempo meio dessas funções e a equação de Schrödinger, em forma vetorial pode ser escrita como:

$$
\frac{d}{d t}|\psi(t)\rangle=-i H(u(t))|\psi(t)\rangle
$$

Na equação acima, $H(u(t))$ é uma matriz n-dimensional que é função de $u(t)$ e que é hermitiana para quaisquer valores de $u$. Por óbvio, usando a terminologia da teoria de controle, $|\psi(t)\rangle$ representa o estado do sistema. Em função da condição de normalização, $\||\psi(t)\rangle \|=1$ para todo $t \in \Re$, a evolução dinâmica do estado do sistema deve ser tal que $|\psi(t)\rangle$ é um vetor sobre a esfera esfera unitária no espaço vetorial complexo $C^{n 18}$.

Uma ampla classe de sistemas de controle quânticos é tal que:

$$
H(u(t))=H_{0}+\sum_{k} H_{k} u_{k}
$$

De fato, como o Hamiltoniano é um observável que está relacionado à energia total do sistema, para uma ampla categoria de sistemas a incorporação de mecanismos adicionais de troca de energia é realizada pela simples soma de um termo à energia total do sistema, ou seja, adicionando um termo ao Hamiltoniano. Considerando também que os termos adicionais estão normalmente vinculados a um campo eletromagnético que pode

17 Assim, para uma descrição estática e minimamente suficiente de um sistema quântico é necessário especificar o espaço de Hilbert subjacente e os observáveis. O estado do sistema somente pode ser determinado por meio de processos de medição. Para uma descrição da dinâmica, é necessário ainda especificar o observável energia que define a evolução temporal dos estados do sistema

18

$\mathrm{Na}$ realidade, o fato de o operador hamiltoniano ser hermitiano garante que o propagador dinâmico do sistema seja unitário, o que faz com que a norma unitária do estado seja preservada para todo $t \in \Re$ 
ser diretamente controlado (podendo inclusive ser desligado), a forma da equação 1.19 é plenamente justificada por seu interesse prático. A classe de sistemas que descreve é também bastante abrangente.

Assim, os sistemas de controle quânticos podem ser modelados como sistemas bilineares:

$$
\frac{d}{d t}|\psi(t)\rangle=-i\left(H_{0}+\sum_{k} H_{k} u_{k}\right)|\psi(t)\rangle
$$

A representação acima, equação 1.20, continua válida nos casos em que um diferente conjunto de Hamiltonianos, $\bar{H}_{k}$, é construído a partir do conjunto inicial que implementa o modelo físico do sistema. Nesses casos, o novo conjunto de Hamiltonianos é fictício. Pode-se até mesmo eliminar o termo responsável pela dinâmica livre, $H_{0}$, o que facilita as análises realizadas para implementar os controles. Define-se, portanto, uma mudança de variáveis relativa aos parâmetros de controle.

$$
\frac{d}{d t}|\psi(t)\rangle=-i\left(\sum_{k} \bar{H}_{k} \bar{u}_{k}\right)|\psi(t)\rangle
$$

As análises realizadas até o momento se referem ao controle de estados puros, $|\psi(t)\rangle$. No entanto, os sistemas quânticos podem ser representados por meio de operadores densidade. Esse método de modelagem de sistemas quânticos é muito importante em função da generalização do conceito de estado dinâmico. Sabe-se que um estado representado por um operador densidade pode ser puro, caso em que também poderia ser representado por vetor no espaço de Hilbert, ou seja, um quet, como também pode ser impuro, caso em que representa uma mistura de estados quânticos puros. Assim, os operadores densidade podem ser utilizados para modelar incertezas relativas ao estado de sistemas quânticos ${ }^{19}$.

A representação de estados por operadores densidade permite tanto a modelagem de incertezas com relação ao estado inicial e a utilização de modelos de evolução dinâmica determinística, quanto a modelagem de incertezas com relação ao estado inicial e a evolução dinâmica estocástica decorrente de utilização de processos de medição ou de acoplamentos do sistema com o ambiente.

Assim, no caso de o sistema quântico ser representado por um operador densidade, a evolução determinística de seu estado pode ser descrita por uma equação de Liouville com o Hamiltoniano dependente dos parâmetros de controle:

$$
\dot{\rho(t)}=[-i H(u(t)), \rho(t)]
$$

19 É também possível utilizar modelos com estados puros para representar incertezas. Esse é o objetivo dos modelos baseados em trajetórias quânticas. No lugar de simular um sistema quântico com operadores densidade, representado por matrizes que apresentam custo computacional elevado, simula-se um sistema puro um grande número de vezes tomando diferentes condições iniciais. Há uma rica discussão teórica a respeito da realidade física das trajetórias quânticas, vide brilhante análise apresentada por Wiseman em (WISEMAN, 2003) 
A estrutura da equação 1.22 garante, assim como a equação 1.18, que evolução dinâmica do estado, representado pelo operador densidade, $\rho(t)$, preserve sua estrutura. Assim, $\rho(t)$ varia no conjunto de operadores positivos, semidefinidos com traço unitário. Esse conjunto possui a estrutura de uma variedade diferenciável com borda. No caso de sistemas dinâmicos bidimensionais, a esfera de Bloch constitui uma excelente representação geométrica do conjunto de estados fisicamente relevantes. A borda do conjunto é composta dos pontos equivalente à condição $\|\rho(t)\|=1$, já seu interior é composto dos estados para os quais a norma do operador densidade é inferior a um ${ }^{20}$.

A solução da equação 1.22 pode ser escrita como:

$$
\rho(t)=X(t) \rho(t) X^{\dagger}(t)
$$

O operador unitário $X(t)$ representa o propagador dos estados do sistema dinâmico representado pelo estado $\rho(t)$. Tal operador é solução da equação de Schrödinger para operadores:

$$
\dot{X}(t)=-i H(u(t)) X(t), X(0)=I
$$

A equação dinâmica para operadores, equação 1.24 possui importância fundamental para o estudo de sistemas de controle quânticos e possui uma interpretação física importante. Uma operação quântica a ser implementada para realizar, por exemplo, qualquer manipulação de informação entre estados quânticos pode ser representada por um determinado propagador unitário $X(t) \in U(n)$. Em condição inicial, $\left|\psi_{0}\right\rangle$ consiste no estado inicial que possui a informação quântica a ser manipulada e o propagador unitário $X(t)$ consiste na operação lógica a ser aplicada no estado inicial com o objetivo de realizar modificações na informação quântica codificada no estado do sistema, levando ao estado final $\left|\psi_{f}\right\rangle$.

$$
\left|\psi_{f}\right\rangle=X\left(t_{f}\right)\left|\psi_{0}\right\rangle
$$

A realização sistemática dessas operações lógicas é, em linhas gerais, o processamento quântico de informação, ou seja, computação quântica. A analogia com o processamento clássico de informação por meio de portas lógicas operando operações booleanas é direta. Uma introdução à área da computação quântica será realizada a seguir.

Assim, o problema de controle pode ser colocado em termos do propagador $X(t)$, tendo por objetivo que a evolução dinâmica do sistema seja equivalente à realização de uma operação lógica de natureza quântica. Ao formular o problema nesses termos genéricos, é possível tanto a solução de problemas de controle para o estado $|\psi\rangle$ quanto a solução de problemas de controle baseados em operadores densidade $\rho$.

20 No caso bidimensional, todos os pontos da borda representam estados puros, no entanto, em dimensões superiores a estrutura da borda é mais complexa, vide XXX 
Geometricamente, o sistema dinâmico da equação 1.24 possui como espaço subjacente um grupo de Lie, o grupo de matrizes unitárias de dimensão $n$, ou seja, a dimensão do sistema quântico. Por se tratar da análise de sistemas dinâmicos em grupos de Lie, a transposição dos métodos da teoria geométrica de controle ocorre de forma transparente.

Note-se finalmente, que, até este ponto, nesta introdução ao problema de controle quântico, foi dada ênfase apenas ao problema de controle determinístico em espaços de dimensão finita. Uma vez que a realização de medições introduz incerteza na evolução dinâmica do sistema, sistemas dinâmicos quânticos que envolvem processos de medição não podem ser modelados usando os métodos determinísticos acima. Como resultado, a análise determinística da dinâmica quântica somente se presta a analisar estratégias de controle em malha aberta.

\subsection{Computação Quântica}

Um pequeno e limitado panorama a respeito da computação quântica será apresentado no Capítulo 2. É importante destacar que a contrução de uma máquina de Turing quântica, ou seja, um computador quântico, depende do desenvolvimento tecnólogico de uma arquitetura de hardware cuja dinâmica seja controlável. A controlabilidade, do ponto de vista da dinâmica, é equivalente à universalidade, do ponto de vista da teoria da computação quântica. 


\section{Pesquisa Bibliográfica}

O campo de pesquisa na área da computação quântica apresenta duas vertentes principais: a parte relacionada ao funcionamento lógico dos sistemas de processamento de informação, que abrange a elaboração e análise de algoritmos assim como o estudo de mecanismos de utilização de sistemas quânticos de informação, e a parte relacionada à implementação física de tais sistemas. Os dois aspectos estão intimamente ligados uma vez que, no processamento quântico, assim como no processamento clássico, o hardware, sistema físico, executa as operações lógicas de manipulação da informação. Sistemas quânticos de dimensão finita ou infinita podem ser utilizados na manipulação de informação, esses últimos tanto de espectro discreto quanto contínuo. O processamento de informação em sistemas quânticos apresenta como principais problemas a serem resolvidos a susceptibilidade do hardware a interferências externas e o controle da evolução dinâmica do estado físico dos elementos de hardware, que é a principal classe de problema abordados neste trabalho.

$\mathrm{Na}$ interface entre a computação quântica e os sistemas dinâmicos encontramos uma área de convergência da teoria da computação, da física, da matemática e da engenharia, com uma grande riqueza de fenônomenos e um vasto potencial para o desenvolvimento de novas tecnologias que serão capazes de modificar o panorama tecnológico global. A utilização de um computador quântico para realizar processamento de informação levará a uma revolução nas tecnologias de criptografia ${ }^{1}$ e poderá trazer modificações profundas na física, na química e suas áreas correlatas em consequência da possibilidade de simular modelos mais reais de sistemas de interesse. Na área da engenharia haverá obviamente uma forte demanda para absorver as novas tecnologias em desenvolvimento com o objetivo de colaborar na construção dos novos computadores quânticos e também com o objetivo de adaptá-las para a solução de outros problemas. Uma forte contribuição da engenharia nesse empenho está relacionada à adaptação das técnicas e dos conceitos da teoria de controle de sistemas para aplicá-las à dinâmica de sistemas de natureza quântica. E é nesse contexto que este trabalho está inserido.

Um sistema quântico fechado de dimensão finita é representado por um sistema de equações diferencias ordinárias sobre o corpo dos números complexos. O espaço de estados é uma variedade diferenciável, a esfera unitária em $\mathbb{C}^{n}$, e os métodos desenvolvidos pela geometria diferencial e pela teoria de controle geométrico são suficientes para abordar o problema de controle em malha aberta relacionado a um sistema fechado.

1 Em 2004, com base no algoritmo AKS ou AKSL, demonstrou-se que o problema de determinar se um número é ou não primo está na classe de complexidade polinomial (AGRAWAL; KAYAL; SAXENA, 2004) apud (MARTINEZ et al., 2015). 
Do ponto de vista da teoria de controle, as unidades fundamentais de hardware quântico devem inicializar e guardar um determinado estado quântico, protegê-lo de interação degradadoras com o ambiente externo e, de forma controlada e com a máxima acuracidade possível, modificar o estado interno do sistema para o estado final desejado 2. O sistema descrito é usualmente representado, em dimensão finita, como uma porta lógica, ou seja, um dispositivo quântico com uma entrada, uma saída e um circuito de conexão que realiza a operação lógica com o estado da entrada, apresentando o resultado na saída. Esta representação é atualmente o maior paradigma da computação quântica e é utilizado tanto para representar algoritmos quanto para modelagem de hardware, especialmente em sistemas fotônicos.

Dada uma representação matemática de um determinado sistema quântico de processamento de informação, do ponto de vista da teoria de controle, o primeiro problema a ser analisado e resolvido é o da controlabilidade dos estados. De sua solução se pode obter o conjunto de estados preparáveis e, a partir desses, o conjunto de estados acessíveis ou alcançáveis.

Para sistemas quânticos de dimensão finita, assim como para sistemas de controle clássicos, a controlabilidade pode ser determinada pela análise da álgebra de Lie dos campos de vetores utilizados para manipular o sistema (D'ALESSANDRO, 2008). Há, no entanto, o suplementar problema relacionado aos processos de medição quânticos, que interferem no estado do sistema. Há diversas análises de controlabilidade disponíveis para esse tipo de sistema na literatura, poucas, no entanto, levam em consideração o problema da medição.

A manipulação de estados em sistemas de dimensão infinita é dificultada por uma série de fatores teóricos. A modelagem de sistemas em espaços de dimensão infinita apresenta problemas de definição de domínio dos operadores lineares que agem espaço de Hilbert subjacente. Como consequência, deve-se buscar a definição de um subespaço denso de tal forma seja comum aos operadores de interesse. A análise de convergência em espaços de dimensão infinita é complexa e os operadores lineares possuem estrutura adicional, por exemplo, em dimensão infinita surgem operadores com espectro contínuo (REED; SIMON, 1980; PRUGOVECKI, 1981; HUANG; TARN, 1983; WU; TARN; LI, 2006).

Neste trabalho pretende-se abordar a controlabilidade de sistemas quânticos de dimensão finita e infinita em malha aberta (D'ALESSANDRO, 2008; PRUGOVECKI, 1981; HUANG; TARN, 1983; WU; TARN; LI, 2006). Posteriormente serão discutidas as perturbações induzidas no modelo pelo processo de medição (BREUER; PETRUCCIONE, 2002; CALLEN; WELTON, 1951; GARDINER; ZOLLER, 2004; JACOBS, 2014;

2 Estes são, de forma simplificada e não completa, os critérios de DiVincenzo (DIVINCENZO, 1994) para a análise de viabilidade de propostas de arquitetura de hardware. 
JACOBS; STECK, 2006; BRUN, 2001; CARMICHAEL, 1990; CARMICHAEL, 1991; WISEMAN; MILBURN, 2010). Os modelos propostos podem ser simulados numericamente tanto em malha aberta, sem medição, utilizando EDOs lineares, quanto em malha fechada a partir das equações dinâmicas que modelam os processos de medição contínua, utilizando equações diferenciais de natureza estocástica (JACOBS; STECK, 2006; JACOBS, 2014; BRUN, 2001; BREUER; PETRUCCIONE, 2002; GARDINER; ZOLLER, 2004; WISEMAN; MILBURN, 2010).

Uma vez estabelecidos modelos matemáticos para sistemas com medição contínua, podem ser estudadas as classes de processos de medição assim como as características das perturbações provocadas no estado do sistema em consequência da dinâmica da medição. Importantes classes de medição a serem estudadas são as medições QND ou BAE, medições eficientes, medições fortes e fracas (CAVES et al., 1980; BRAGINSKY; KHALILI, 1995; JACOBS, 2014; WISEMAN; MILBURN, 2010). Importantes configurações de medição já foram implementadas em laboratório com sucesso, por exemplo, Wiseman (WISEMAN, 1995), em 1995, propôs um método de controle para realimentar o sistema monitorado e anular, nos limites estabelecidos pela teoria quântica, os efeitos de perturbação do sistema.

\subsection{Do Processamento Quântico à Teoria de Controle}

Há uma grande quantidade de trabalhos desenvolvidos sobre o processamento quântico de informação. Do ponto de vista do desenvolvimento de uma nova tecnologia, é importante entender os novos fenômenos físicos que se apresentam, assim como a escala em que se apresentam problemas já conhecidos. É importante também entender as abstrações que se pretendem implementar fisicamente. Há novos fenômenos a serem compreendidos pela engenharia assim como uma nova escala de trabalho. E os modelos abstratos são relacionados a um tipo diferente de autômato, a máquina de Turing quântica.

Os fenômenos quânticos não são desconhecidos da engenharia, principalmente na área da computação. Os processos de construção de circuitos eletrônicos em semicondutores estão em constante desenvolvimento, principalmente em termos de miniaturização. No entanto, o limite dessa miniaturização é definido quanticamente. De forma mais importante, o funcionamento dos semicondutores é desenvolvido a partir de modelos quânticos. O laser é outro exemplo de tecnologia essencial à engenharia e que possui natureza quântica. No entanto, a engenharia deverá absorver novos elementos teóricos da física quântica no desenvolvimento de tecnologias para a modelagem e fabricação de hardware quântico. Trata-se também nesse caso de uma mudança de escala. No caso dos semicondutores e também do laser, existe um conjunto de elementos que se comportam quanticamente 
e que dão origem a fenômenos detectáveis macroscopicamente, diferenças de potencial elétrico, correntes elétricas e intensidades luminosas por exemplo. No entanto, para que um sistema físico seja viável para aplicação em processamento quântico de informação, é necessário que o estado quântico do sistema seja diretamente manipulável e monitorável. Deixa-se de trabalhar na escala da física do estado sólido, em que um grande número de partículas dá origem a um determinado fenomeno que é tratado estatisticamente, para trabalhar na escala atômica. ${ }^{3}$ Manipulando, por exemplo, o spin de um elétron ou do núcleo de um átomo, construindo circuitos ópticos capazes de operar com apenas um fóton, ou manipulando pares de elétrons de cooper em supercondutores (NIELSEN; CHUANG, 2000).

Um dos significados dessa transição para a engenharia é que, nessa nova área de pesquisa, as teorias da mecânica e da eletrodinâmica quânticas, antes praticamente restritas à Física, serão incorporadas à engenharia em mesmo nível de profundidade que a mecânica analítica e o eletromagnetismo, por exemplo. Não é possível nem mesmo entender o princípio de funcionamento de um sistema de processamento quântico de informação sem domínio da estrutura matemática e dos axiomas da física quântica (NIELSEN; CHUANG, 2000; DEUTSCH, 1985). É importante notar que essa transição não é trivial, pois a mecânica quântica apresenta uma estrutura de funcionamento que é considerada não intuitiva. Os padrões lógicos que funcionam perfeitamente na modelagem de sistemas mecânicos e eletromagnéticos deixam de funcionar na escala quântica. Não é exagero afirmar que existe uma nova estrutura lógica subjacente à física quântica (BIRKHOFF; NEUMANN, 1936). É justamente com base em fenômenos quânticos que não têm paralelo na física clássica que os mecanismos de manipulação de informação propostos por Deutsch (DEUTSCH, 1985) são capazes de demonstrar o grande potencial do processamento quântico de informação.

Classicamente o modelo de Turing para a computação é utilizado como paradigma para estudo da complexidade de algoritmos e também responder a questões fundamentais sobre classes de problemas computáveis e sobre implicações do modelo de computação para a lógica e os fundamentos da matemática. A máquina de Turing é um dispositivo discreto que funciona a partir da definição de uma máquina de estados finita capaz de operar com uma lista finita de instruções e de símbolos em um dispositivo de memória linear. O processamento quântico de informação pode ser modelado por meio de uma máquina de Turing quântica (BENIOFF, 1995), (LAGANA; LOHE; SMEKAL, 2009). Implementações específicas de computadores quânticos podem induzir naturalmente modelos de máquinas de Turing distintos, mais adaptados a realizar análises de complexidade computacional, como em (MATSUI, 2004). Para além do modelo computacional baseado

3 Em circuitos eletrônicos associados a junções de Josephson e em outros sistemas quânticos desenvolvidos na plataforma de semicondutores, trabalha-se em uma escala intermediária, denominada mesoscópica. 
em máquinas de Turing, pode-se destacar inicialmente dispositivos de processamento de informação especialmente construídos para um fim específico. No caso de simulação de sistemas quânticos, (MATTHEWS, 2012) reportam avanços utilizando sistemas fotônicos para simular a evolução temporal conjunta de agrupamentos de bosons ou mesmo de fermions. O sistema proposto oferece grande escalabilidade o que torna mais próxima a realização física de um simulador quântico com poder computacional compatível com os atuais supercomputadores clássicos. Para a categoria de problemas de otimização foi proposta a arquitetura de otimização quântica adiabática, (KARIMI et al., 2011). Nessa linha tecnológica, a empresa canadense D-Wave Systems sustenta ter criado o primeiro computador quântico do mundo baseado em supercondutores, em arquitetura de otimização adiabática (D-WAVE-SYSTEMS, 2012).

Fisicamente há propostas de computadores quânticos baseado em processos físicos reversíveis (LADD et al., 2010) e outros baseados em processos irreversíveis. A categoria de processamento reversível inclui a maior parte dos computadores baseados em circuitos quânticos, nos quais ocorre o encadeamento de portas quânticas que implementam um conjunto universal de operações unitárias (DEUTSCH, 1985), (BENIOFF, 1982), (BENIOFF, 1997), (BENIOFF, 1998), (OZAWA, 1998) (NISHIMURA; OZAWA, 2005). Para a arquitetura baseada em circuitos, já existe um processo de otimização de circuitos quânticos. O circuito ótimo pode ser determinado passo a passo por meio de um processo equivalente à busca de uma trajetória extremal em um espaço de geometria curva (NIELSEN, 2005), (NIELSEN et al., 2006). Arquiteturas tolerantes a falhas, conforme proposição inicial de Preskill, (PRESKILL, 1997), já chegam a oferecer alternativas para dispositivos com taxas de falha de até três por cento (KNILL, 2005; RAUSSENDORF; HARRINGTON, 2007).

Na outra categoria, pode-se destacar os computadores baseados em processos de medição. Nesses dispositivos, um sistema quânticos de várias partículas é iniciado em um determinado estado emaranhado e o processamento de informação se dá por meio da medição de determinadas partículas do conjunto. A arquitetura proposta já foi testada fisicamente demonstrando-se ser realista. Há também a possibilidade de se implementar processos de computação tolerante a falhas, o que confere robustez ao modelo de computação quântica proposto. Detalhes podem ser vistos em (MILNE; KOROLKOVA, 2012), (BRIEGEL et al., 2009) e (WALTHER et al., 2005). Outras propostas podem ser encontradas em (HOSTEN et al., 2006), (OLIVER, 2011).

Há propostas para estruturar modelos de computação contínuos, capazes de operar diretamente com números reais ou complexos, sendo esta, supostamente, o fundamento ideal para a análise de algoritmos que modelem sistemas contínuos (TRAUB, 1999). (RODó, 2011), (LLOYD; BRAUNSTEIN, 1999)

Em paralelo ao modelo clássico de Turing, encontra-se o cálculo lambda, criado 
por Alonzo Church, que se consolidou como fundamento para diversas linguagens de computação, desde o LISP até o FORTRAN. De acordo com a conjetura de ChurchTuring, todo e qualquer algoritmo pode ser implementado por uma máquina de Turing e, equivalentemente, por uma função de cálculo lambda. A definição de cálculo lambda também pode ser feita para algoritmos quânticos e a equivalência entre funções nele definidas e a máquina de Turing quântica podem ser verificadas em (TONDER, 2004). A partir da definição proposta se poderia investigar a utilidade de linguagens de programação quânticas e se realizar comparações com o modelo de circuito quântico (MISZCZAK, 2010), (MAYMIN, 1997).

Um grande avanço no desenvolvimento da computação quântico é devido (SHOR, 1994), que apresentou um algoritmo quântico capaz de realizar a fatoração de números inteiros a um custo polinomial. A comunidade científica da área da teoria da computação, em particular da área de criptologia, deparou-se com um resultado que vai de encontro a premissas e conjecturas fortes em suas áreas de atuação. A criptologia assumia como premissa que códigos criptográficos baseados em números primos grandes seriam potencialmente seguros. Essa suposição era baseada na inexistência de algoritmos clássicos eficientes para a fatoração de números inteiros e em uma conjectura de que seriam inexistentes. ${ }^{4}$ O algoritmo de Shor representou para teoria da computação uma mudança estrutural.

Em sua forma de apresentação usual, no modelo de computação baseado em circuitos, o processamento quântico de informação é baseado na manipulação de um conjunto de sistemas quânticos de dois níveis denominados qubits. Um algoritmo é representado por uma sequência de operações, denominadas portas quânticas, que modificam dinamicamente um ou mais qubits. A saída do algoritmo é dada pelo resultado da medição do conjunto de qubits (DIVINCENZO, 1994; NIELSEN; CHUANG, 2000). Há também arquiteturas para codificar informação quântica em sistemas quânticos de dimensão infinita utilizando variáveis contínuas (LLOYD; BRAUNSTEIN, 1999). Um importante conceito na computação quântica é o de universalidade. Um sistema de computação quântica é dito universal quando sua estrutura é capaz de aproximar com precisão arbitrária qualquer transformação unitária no espaço de estados no qual é modelado o sistema composto pelo conjunto de qubits (DIVINCENZO, 1994; NIELSEN; CHUANG, 2000).

Os computadores quânticos são, portanto, sistemas dinâmicos representáveis em um espaço de Hilbert adequado cuja evolução é dada por uma equação diferencial linear denominada equação de Schrödinger. Os operadores lineares a partir dos quais a equação se contrói são do tipo auto adjuntos e formam uma álgebra não comutativa. Os grupos de transformações induzidos pela equação no espaço de estados estão contidos no grupo

$4 \quad$ Posteriormente, demonstrou-se que a classe de complexidade clássica da fatoração em números primos é polinomial (AGRAWAL; KAYAL; SAXENA, 2004) apud (MARTINEZ et al., 2015). 
Tabela 1 - Comparação entre diferentes sistemas quânticos, mostrando seus tempos característicos.

\begin{tabular}{|l|l|l|l|}
\hline \hline Sistema & $\tau_{Q}$ & $\tau_{o p}$ & $n_{o p}=\lambda^{-1}$ \\
\hline Spin nuclear & $10^{-2}-10^{8}$ & $10^{-3}-10^{-6}$ & $10^{5}-10^{14}$ \\
Spin do eletron & $10^{-3}$ & $10^{-7}$ & $10^{4}$ \\
Ions aprisionados $\left(\mathrm{In}^{+}\right)$ & $10^{-1}$ & $10^{-14}$ & $10^{13}$ \\
Eletron - Au & $10^{-8}$ & $10^{-14}$ & $10^{6}$ \\
Eletron - GaAs & $10^{-10}$ & $10^{-13}$ & $10^{3}$ \\
Quantum dot & $10^{-6}$ & $10^{-9}$ & $10^{3}$ \\
Cavidade óptica & $10^{-5}$ & $10^{-14}$ & $10^{9}$ \\
Cavidade de microondas & $10^{0}$ & $10^{-4}$ & $10^{4}$ \\
\hline \hline
\end{tabular}

de Lie das transformações unitárias do espaço de Hilbert subjacente (HUANG; TARN, 1983; D'ALESSANDRO, 2008). Em conclusão, dado um sistema quântico modelado em um espaço de Hilbert qualquer, os conceitos de universalidade e de controlabilidade são equivalentes (DIVINCENZO, 1994; D'ALESSANDRO, 2008; WU; TARN; LI, 2006)

\subsection{Desafios Tecnológicos}

Em meio aos desenvolvimentos teóricos da computação quântica que estruturaram tanto os conceitos de máquina de Turing quântica e de circuitos quânticos, quanto os diversos algoritmos quânticos capazes de resolver problemas computacionais com vantagens sobre sistemas clássicos, iniciou-se uma corrida pela construção dos computadores quânticos (CHUANG et al., 1998; KNILL, 2010; LADD et al., 2010). A Tabela 1 apresenta tempos de referência para comparação de diferentes sistemas que são candidatos à implementação física de qubits. São apresentados o tempo de decoerência, $\tau_{Q}$, tempo de execução de operações que compõem as portas quânticas, $\tau_{o p}$, e uma estimativa do número de operações lógicas que poderiam ser executadas continuamente até que a interação com o ambiente destruísse a informação quântica codificada fisicamente, $n_{o p}=\tau_{Q} / \tau_{o p}$. A tabela foi reproduzida de (NIELSEN; CHUANG, 2000) e deve ser analisada com dupla cautela. Inicialmente se trata de uma referência de 2000 e, em segundo lugar, os próprios autores alertam que esses são apenas alguns parâmetros físicos relevantes. Assim, a análise dos dados pode fornecer uma boa perspectiva a respeito das dificuldades físicas na implementação de qubits, mas é apenas um conjunto de dados limitado. Há outros parâmetros físicos relevantes assim como há outras possibilidades de implementação física de qubits.

As dificuldades enfrentadas pela comunidade científica no projeto e na construção de dispositivos capazes de implementar fisicamente sistemas quânticos de dois níveis colocam em perspectiva outra importante área de pesquisa relacionada à física quântica teórica, que lida com sistemas abertos. Em sistemas fechados, idealizações teóricas que 
consideram sistemas isolados sem troca de energia e isentos de qualquer interação com seu meio ambiente, o estado do sistema não é perturbado por nenhuma forma de ruído. Nessas condições, é possível imaginar um espaço de Hilbert $n$-dimensional representando um sistema quântico de $n$ qubits controlável, e composto adicionalmente de mecanismos de inicialização e de medição dos qubits. Um sistema de tal tipo é suficiente para modelar o processamento de informação quântica em até $n$ qubits. Nessas condições, é possível imaginar um sistema de processamento quântico de informação baseado em uma arquitetura de controle em malha aberta (D'ALESSANDRO, 2008). Essa idealização é importante para a teoria da computação quântica, pois lhe fornece um modelo estrutural a partir do qual suas construções podem ser desenvolvidas.

No entanto, dispositivos reais não são, nem de forma aproximada, sistemas quânticos fechados e sua interação com o meio ambiente é extremamente nociva à representação da informação em seu estado dinâmico. Os efeitos da interação com o meio ambiente podem ser agrupados em dois tipos de natureza diferentes, mas interdependentes, a dissipação e a decoerência (GARDINER; ZOLLER, 2004; JACOBS, 2014; WISEMAN; MILBURN, 2010; CARMICHAEL, 1990; CARMICHAEL, 1991; BREUER; PETRUCCIONE, 2002). Como ambas estão relacionadas a escalas de tempo distintas e a decoerência possui em geral uma escala de tempo menor, é comum na literatura encontrá-la com mais frequência, representando às vezes de forma genérica, as interações indesejadas com o meio ambiente. Na Tabela 1, por exemplo, encontramos apenas uma referência à decoerência.

Na Figura 1, encontramos uma representação gráfica, com dados mais atualizados, sobre a tendência de evolução da arquitetura de hardware baseada em circuitos quânticos, algumas vezes denominada eletrodinâmica quântica de circuitos ou circuit quantum electrodynamics, circuit-QED, ilustração extraída de (GIRVIN, 2014). Essa arquitetura de hardware quântico possui uma estrutura interessante (BLAIS et al., 2004; SCHUSTER, 2007; GIRVIN, 2014), pois os sistemas quânticos são construídos em uma escala diferente dos sistemas mencionados na Tabela 1, escala esta denominada mesoscópica (JACOBS, 2014; WISEMAN; MILBURN, 2010). Estes circuitos são projetados e construídos em pastilhas de silício e utilizam ilhas de supercondutores formando junções de Josephson. Envolvem elementos passivos como indutores e capacitores interagindo com os modos quânticos da junção de Josephson por meio de guias de ondas, vide por exemplo a didática exposição de Schuster descrevendo a arquitetura de circuitos em QED e ilustrando seus detalhes construtivos chegando a apresentar as receitas para o desenho dos circuitos em pastilhas de silício (SCHUSTER, 2007). Notemos na Figura 1, que os dispositivos representados estão no limiar estabelecido pelos códigos de correção de erro para viabilizarem a construção de sistemas de processamento quântico de informação realísticos.

A arquitetura de circuitos em QED é muito importante para a engenharia, pois se baseia na tecnologia de semicondutores, que possui décadas de desenvolvimento con- 
Figura 1 - Lei de Schoelkopf

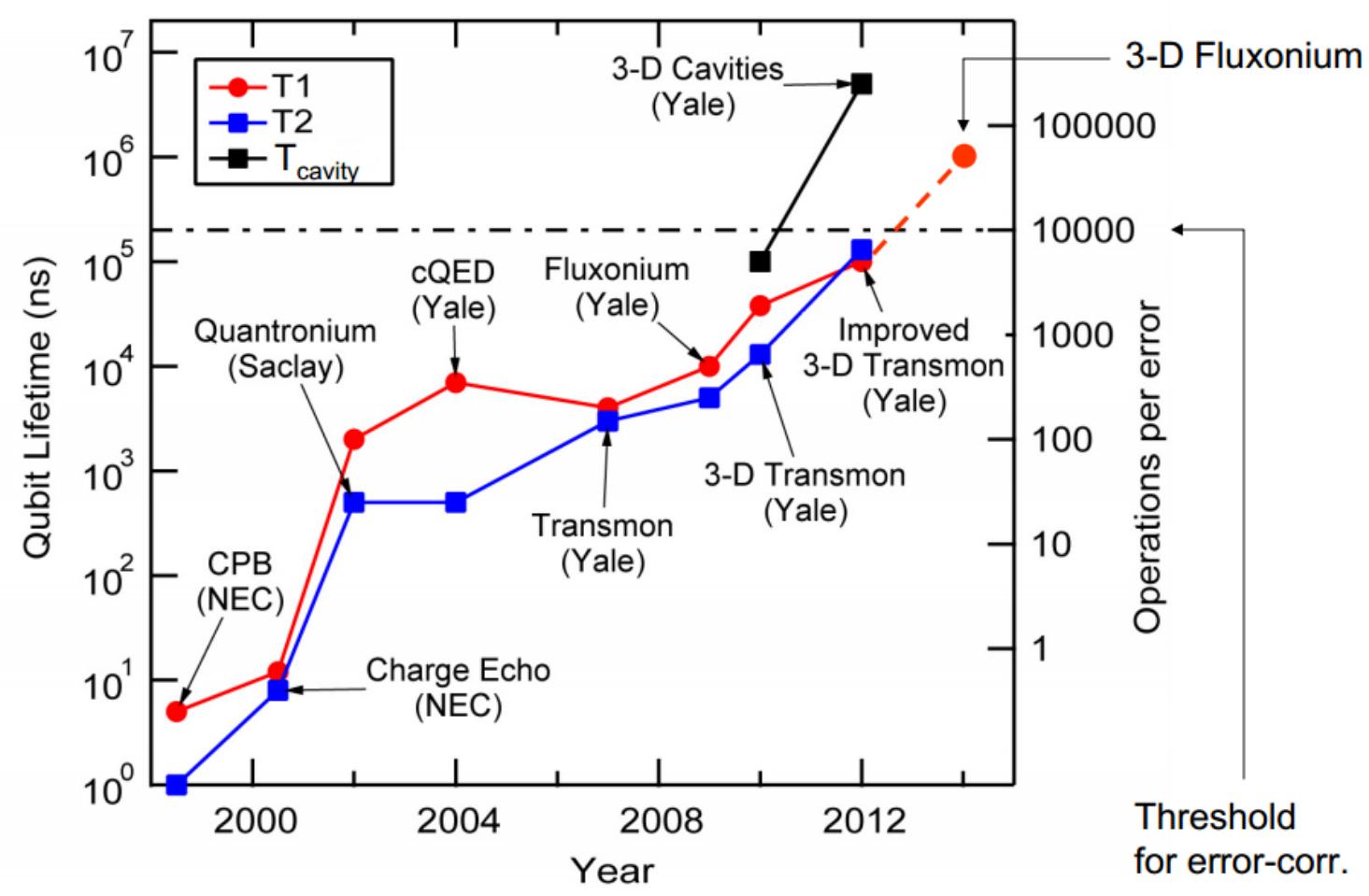

tínuo. É amplamente estudada e tem seus fenômenos bem explorados e documentados. Como é uma tecnologia com intensa aplicação industrial, recebe contínuos investimentos o que nos permite dizer que é uma das tecnologias que a engenharia domina em maior profundidade. Além do potencial como candidata a compor uma arquitetura de hardware para processamento quântico de informação, a tecnologia de circuitos em QED poderá estruturar outros tipos de dispositivos. No entanto, deve-se notar que os dispositivos que implementam os qubits funcionam em temperaturas da ordem de $100 \mathrm{mK}$ (SCHUSTER, 2007).

É também importante mencionar que os sistemas quânticos desenvolvidos por meio da engenharia de circuitos em QED possui modelos matemáticos que são similares em estrutura aos modelos de sistemas contruídos a partir da óptica quântica, no estudo da eletrodinâmica quântica em cavidades ópticas (WISEMAN; MILBURN, 2010; GARDINER; ZOLLER, 2004; BREUER; PETRUCCIONE, 2002; BLAIS et al., 2004; BLAIS et al., 2007; CARMICHAEL, 1991; CARMICHAEL, 1990; PURI, 2001). Muitos desenvolvimentos importantes da física quântica realizados no contexto da óptica quântica, a partir de idealizações iniciais puramente teóricas (BRAGINSKY; KHALILI, 1995; CAVES et al., 1980; CAVES; MILBURN, 1987; CARMICHAEL, 1991; CARMICHAEL, 1990; WISEMAN; MILBURN, 1993; WISEMAN; MILBURN, 1994; WISEMAN, 1994; WISEMAN, 1995; WALLS; MILBURN, 2008).

A física quântica absorveu o desenvolvimento de teorias sobre sistemas quânticos abertos, desde os desenvolvimentos pioneiros de Senitzky e de Redfield por volta dos anos 
1960. Concomitantemente, a área da mecânica estatística realizou uma importante contribuição ao relacionar as flutuações observadas em elementos dissipativos de circuitos elétricos à preservação da distribuição de Boltzmann. A incorporação de estruturas dissipativas na física quântica é uma tarefa não trivial. A flutuação que está associada aos processos dissipativos presentes nos domínios quânticos é responsável pela preservação do princípio da incerteza de Heisenberg (CALLEN; WELTON, 1951; CARMICHAEL, 1991; CARMICHAEL, 1990; GARDINER; ZOLLER, 2004).

A dissipação e a decoerência são processos distintos. O significado dessa expressão deve ser entendido com algumas ressalvas. Na realidade, tanto a decoerência quanto a dissipação são consequências da dinâmica de sistemas abertos e ocorrem simultaneamente, em maior ou menor grau, a depender das características do sistema e de seu acoplamento com o meio que o envolve. Um dos elementos fundamentais para o desenvolvimento da decoerência é o emaranhamento entre o sistema de interesse e o meio ambiente em seu entorno. Dado que o emaranhamento é um fenômeno intrinsecamente quântico, que decorre da estrutura de composição de sistemas, a decoerência é um fenômeno genuinamente quântico. Já adiantamos que a incorporação da dinâmica de processos de medição é realizada via teoria de sistemas dinâmicos abertos, o que, de certa forma é esperado, pois em qualquer processo de medição deve haver algum tipo de troca. Dessa forma, como na teoria de sistemas fechados se assume que os processos de interação são nulos, os experimentos de medição não são compatíveis com uma teoria de sistemas fechados. (BREUER; PETRUCCIONE, 2002; JACOBS, 2014; WISEMAN; MILBURN, 2010).

A necessidade de realizar um tratamento sistemáticos dos efeitos deletérios da interação do ambiente levou à criação de um padrão de operações que modelam os efeitos dissipativos do ambiente, vide (NIELSEN; CHUANG, 2000). Com a ocorrência de um erro no processamento de informação seu resultado não será fidedigno. Com o objetivo de demonstrar da viabilidade de se construir um computador quântico capaz de executar algoritmos e obter respostas corretas, foram desenvolvidos códigos de correção de erros, que foram utilizados para estruturar arquiteturas de circuitos tolerantes a falhas (PRESKILL, 1997; SHOR, 1995; KNILL, 2005; NIELSEN; CHUANG, 2000). O surgimento de uma dinâmica irreversível a partir de um modelo da interação do sistema de interesse com seu meio ambiente, baseado em equações conservativas, pode compreendido a partir de uma análise apresentada por (GIRVIN, 2014) para o caso de circuitos em QED.

Os principais requisitos de projeto para a construção de um computador quântico funcional são conhecidos na literatura como os critérios de DiVincenzo e estabelecem, com base nos fatores limitadores impostos pela natureza física dos dispositivos, os parâmetros operacionais mínimos a serem atendidos. Estabelecem que é necessário (DIVINCENZO, 2000; LADD et al., 2010):

1. Um conjunto de sistemas quânticos de 2 níveis, qubits, com Hamiltonianos bem 
definidos e aptos a serem compostos por produto vetorial para a geração de sistemas quânticos de processamento de $n$ qubits.

2. Um sistema de inicialização para colocar o sistema em um estado quântico desejado.

3. Um conjunto de operações lógicas com tempos de execução pequenos em comparação com a escala de tempo de decoerência do dispositivo.

4. Um conjunto universal de operações lógicas (obviamente, este critério e o anterior devem ser analisados concomitantemente).

5. Um sistema de medição independente para cada qubit.

6. Um sistema de interligação entre qubits estacionários e qubits em movimento.

7. Um canal de transmissão para a transmissão de qubits entre diferentes localidades

Os cinco primeiros critérios estão relacionados à computação quântica em si, enquanto os dois seguintes estão relacionados à área de comunicação quântica (DIVINCENZO, 2000). Neste trabalho não analisaremos diretamente as questões relacionadas à comunicação quântica. O foco principal desta monografia é a análise do requisito 4, que está relacionada à análise de controlabilidade de um sistema dinâmico quântico. Analisaremos também a relação entre a dinâmica da medição e a dinâmica do sistema de interesse, assim como alguns aspectos relacionados aos critérios 3 e 5 .

\subsection{Controlabilidade de sistemas quânticos}

A análise da controlabilidade de sistemas quânticos a ser realizada no Capítulo 3 é baseada em um modelo físico de sistema fechado. A dinâmica expressa pela equação de Schrödinger é representada por um operador linear auto adjunto definido em um espaço de Hilbert. Há diferenças fundamentais em relação aos sistemas dinâmicos clássicos que são encontrados nas aplicações em engenharia. Os sistemas de controle mais comuns são definidos em espaços de estados de dimensão finita (CRUZ, 1996; KIRK, 2004; DAVIS; VINTER, 1985; KAILATH, 1980; LEITãO; BAUMEISTER, 2014; LEVINE, 2009; AGRACHEV; SACHKOV, 2004; JURDJEVIC, 1997). Já os sistemas quânticos em geral são definidos em espaços de dimensão infinita. É certo que existem diversos sistemas físicos que se comportam quanticamente e podem ser modelados em dimensão finita, por exemplo, sistemas que modelam o spin de partículas ou conjuntos de partículas (elétrons, núcleos atômicos, entre outros). No entanto, sistemas aparentemente simples e de importância fundamental nas aplicações somente podem ser modelados em espaços de Hilbert de dimensão infinita, por exemplo, uma partícula livre em uma dimensão espacial, um oscilador harmônico quântico, sistemas com momento angular (SAKURAI, 
1994; COHEN-TANNOUDJI; DIU; LALOё, 1977; CARMICHAEL, 1991; WALLS; MILBURN, 2008; SCULLY; ZUBAIRY, 1997). Na classe dos sistemas em dimensão infinita, podemos separar aqueles que apresentam espectro contínuo dos que apresentam espectro discreto. Ao adicionar um potencial quadrático ao Hamiltoniano de uma partícula livre, obtemos um sistema de dimensão infinita com espectro discreto, mesmo considerando que os operadores auto adjuntos $X$, posição, e $P$, momento linear, utilizados na composição do Hamiltoniano do sistema têm espectro contínuo. O oscilador harmônico possui uma importância fundamental na física quântica, tendo em vista que a quantização do campo eletromagnético pode ser realizada a partir de uma modelo que toma por elemento fundamental um conjunto de osciladores harmônicos (PURI, 2001; CARMICHAEL, 1991; WALLS; MILBURN, 2008; SCULLY; ZUBAIRY, 1997; WISEMAN; MILBURN, 2010).

A dinâmica estabelecida pela equação de Schrödinger é linear, dito de outra forma, é uma equação diferencial de evolução baseada em um operador linear auto adjunto definida em um espaço linear completo com produto interno hermitiano (REED; SIMON, 1980; PRUGOVECKI, 1981). Encontra-se em utilização na física quântica uma nomenclatura que define sistemas de natureza não linear. No entanto, essa terminologia não tem o mesmo significado que adquiriu na área de sistemas dinâmicos e na teoria clássica de controle. Encontramos a denominação caracterizando sistemas quânticos cuja dinâmica é determinada por Hamiltoniano que possui similar clássico. O sistema quântico é dito não linear quando a função Hamiltoniana clássica equivalente dá origem a um sistema dinâmico não linear. Assim, por exemplo, no caso de um filtro de Kerr, usado em óptica quântica, que tem dinâmica definida pelo Hamiltoniano $H_{k}=\left(X^{2}+P^{2}\right)^{2}$, não é porque o Hamiltoniano do sistema contém termos de quarta ordem que a equação de Schrödinger deixará de ser linear. ${ }^{5}$

Um sistema de controle quântico, conforme definição que adotamos neste trabalho, é uma sistema de controle bilinear definido pela equação de Schrödinger em um espaço de Hilbert. Quando o espaço de estados do sistema quântico tem efetivamente dimensão finita, os métodos desenvolvidos para tratar sistemas de controle clássicos em grupos de Lie são suficientes para abordar as questões relativas à controlabilidade (D'ALESSANDRO, 2008; ALBERTINI; D"ALESSANDRO, 2003; RAMAKRISHNA et al., 1995; HUANG; TARN, 1983). Note-se, em particular, o excelente trabalho sobre o tema em (LISBOA, 2015), abordando a definição do rpoblema dinâmico, os critérios de controlabilidade, a utilização das técnicas de controle ótimo e a definição de fatores de mérito para a comparação entre técnicas e para a análise de dispositivos e arquiteturas reais. No contexto da teoria de controle geométrica clássica uma importante relação é feita entre determinadas

5 Há referências na literatura a respeito de uma equação de Schrödinger efetivamente não linear, no entanto, não tenho conhecimentos para descrever os fenômenos que essa equação supostamente modelararia, ou para afirmar que se trata de um modelo teórico que não encontra aplicação prática no sentido de modelar fenômenos físicos fundamentais. 
álgebras de campos de vetores, construídas a partir dos campos de vetores livres e dos campos de vetores de controle e o grupo de Lie gerado pelo conjuntos dos campos de vetores anteriores, que geram a dinâmica do sistema no espaço de estados (JURDJEVIC, 1997; AGRACHEV; SACHKOV, 2004). Na linguagem da física quântica, os campos de vetores têm papel desempenhado pelos Hamiltonianos, que são operadores lineares auto adjuntos, que também estão relacionados a uma álgebra, por meio da operação de comutação (SAKURAI, 1994; PRUGOVECKI, 1981; REED; SIMON, 1980; DAVIES, 1976). O grupo de transformações do espaço de estados, no caso de sistemas quânticos fechados em dimensão finita, é o grupo das transformações unitárias do espaço, $U(n)$, ou o grupo das transformações unitárias especial, $S U(n)$, vide (PRESKILL, 1997; D'ALESSANDRO, 2008; RAMAKRISHNA et al., 1995). Nos sistemas quânticos, assim como nos sistemas clássicos, a dinâmica pode estar restrita a um subgrupo do grupo geral que age no espaço de estados de $n$ dimensões. No caso dos sistemas quânticos, é importante destacar as subalgebras de operadores que comutam, tendo em vista que esses conjunto possui uma estrutura especial a partir da qual se pode fazer uma relação com os sistemas de controle clássicos (PRUGOVECKI, 1981; SAKURAI, 1994), nesse contexto, a terminologia conjunto completo de observáveis que comutam é comum. Ao longo do trabalho poderemos empregar a nomenclatura álgebra $Q N D$, para denominar uma álgebra de operadores que comutam. O significado desse termo ficará claro nos Capítulos 3 e $4 .{ }^{6}$ A aplicação das técnicas de controle ótimo leva a bons resultados também quando aplicada aos sistemas de controle quânticos definidos em espaços de dimensão finita. A técnica de decomposição de grupos de Lie fornece ferramenta útil na solução de problemas e na construção de uma imagem geométrica da dinâmica dos sistemas de controle quânticos. Em dimensão finita, os grupos de Lie são compactos, assim qualquer operador de propagação de um sistema dinâmico definido em um grupo de Lie pode ser escrito como uma composição de um número finito de elementos do grupo de transformações, um resultado muito útil para a engenharia de controle (D'ALESSANDRO, 2008; LISBOA, 2015). ${ }^{7}$ Um problema fundamental em computação quântica está relacionado à otimização de arquiteturas de hardware desenvolvidas em plataformas de dimensão finita. Dado um algoritmo quântico qualquer de $n$ qubits, sua implementação e execução são equivalentes a um operador unitário de evolução $U$ em $S U\left(2^{n}\right)$. Utilizando ferramentas de controle ótimo e geometria diferencial (NIELSEN et al., 2006) desenvolveram um método para estimar o número de

6 Em sistemas de medição do tipo quantum nondemolition, ou QND, o observável, que também é um operador auto adjunto, comuta com os termos do Hamiltoniano. Assim, generalizando o significado para sistemas dinâmicos quânticos, um sistema dinâmico com medição é do tipo QND, quando tanto seus observáveis quanto seus Hamiltonianos pertencem à mesma álgebra QND

7 Não conhecemos a estrutura dos grupos de Lie em dimensão infinita, nem do caso particular referente aos grupos de transformações unitárias. Encontramos, no entanto, na referência (WU; TARN; LI, 2006), apontamentos em relação à dificuldade matemática de se abordar essa questão, em conjunto com a questão do domínio dos operadores não limitados. É possível que também em dimensão infinita, solucionados os problemas referentes à convergência de estados e de operadores, a técnica de decomposição de grupos possa apresentar bons resultados. 
portas quânticas necessário para a implementação de uma determinada operação $U$, no espaço de estados $S U\left(2^{n}\right)$.

No caso dos sistemas de controle quântico em dimensão infinita, enfrentamos tanto problemas analíticos, relacionados à convergência nos espaços de estados e nos espaços das transformações lineares desses espaços, quanto problemas de definição de domínio, relacionados aos operadores lineares não limitados, que não estão definidos para todos os elementos dos espaços de estados (REED; SIMON, 1980; PRUGOVECKI, 1981; HUANG; TARN, 1983; WU; TARN; LI, 2006). É importante destacar que mesmo em dimensão infinita, trabalhando com operadores não limitados, a conexão entre a álgebra de operadores lineares auto adjuntos e o grupo de transformações unitárias do espaço é garantido pelo teorema de Stone (REED; SIMON, 1980; PRUGOVECKI, 1981). Uma primeira tentativa de transpor ao domínio quântico os métodos algébricos baseados na teoria de Lie, (HUANG; TARN, 1983) utilizaram como conjunto de vetores denso no espaço de Hilbert e definido para todos os operadores de interesse, os vetores analíticos (NELSON, 1959) apud (HUANG; TARN, 1983). No entanto, tendo em vista que os vetores analíticos limitam a dimensão do grupo de transformações que pode ser gerado no espaço de estados, os modelos de sistemas de controle quânticos baseados nessa técnica não podem dar origem a sistemas que geram grupos de transformações de dimensão infinita (HUANG; TARN, 1983). Em uma natural generalização, o conjunto de vetores diferenciáveis foi também analisado com o mesmo objetivo, com bons resultados (WU; TARN; LI, 2006). Assim, sob determinadas condições, com base nos resultados encontrados em (WU; TARN; LI, 2006) é possível que sistemas de controle quânticos gerem grupos de transformações de dimensão infinita no espaço de Hilbert, no entanto, os autores não estabelecem condições para que o grupo de transformações gerado seja denso no espaço de estados. As referências (HUANG; TARN, 1983; WU; TARN; LI, 2006) será analisadas mais detalhadamente no Capítulo 3. Um destaque importante a ser feito com relação aos exemplos apresentados em (WU; TARN; LI, 2006) é que todos eles são não lineares. Os próprios autores dão destaque a esse fato, que nos parece importante.

No contexto de sistemas quânticos lineares, no sentido explicitado acima, ao abordar apenas a dinâmica da média das variáveis que compõem o espaço de estados, é possível estabelecer uma forte analogia com os sistemas lineares clássicos. Mostra-se que vale nesse contexto uma versão modificada do critério de Kalman para controlabilidade, assim como versões do filtro de Kalman-Bucy e do regulador linear quadrático, assim como adaptações de variados resultados da área de controle robusto (WISEMAN; MILBURN, 2010; JAMES; NURDIN; PETERSEN, 2007) É importante destacar que, nesse caso descrito, a controlabilidade não significa que um estado arbitrário pode ser gerado no espaço de Hilbert. Apenas significa que as médias de observáveis podem ser levadas a valores arbitrários, um resultado muito mais fraco, vide importantes observações em (WISEMAN; MILBURN, 2010). Outros resultados importantes a respeito da controlabilidade 
podem ser encontrados em (BLOCH; BROCKETT; RANGAN, 2006; CHAMBRION et al., 2009). Bloch et al. analisam a controlabilidade baseada em uma decomposição do espaço de estados em subespaços encaixados e Chambrion et al. analisam a controlabilidade de sistemas discretos com base no truncamento do espaço de dimensão infinita em um subespaço de dimensão finita, realizando uma aproximação de Galerkin, muito comum em métodos numéricos de solução de equações diferenciais parciais que ocorrem em engenharia.

\subsection{Medição, Filtragem e Controle}

A incorporação da dinâmica adicional referente aos processos de medição ao modelo de um sistema dinâmico quântico é uma tarefa não trivial, conforme se pode verificar pela análise da Seção 2.2. A dinâmica de um sistema quântico aberto é em geral não conservativa, portanto, os operadores de evolução no espaço de estados deixam de ser unitários. O estado de um sistema quântico aberto é normalmente descrito por um operador densidade. A dinâmica de evolução do operador densidade deve garantir que, em todo instante $t>0$, a representação do sistema pelo operador densidade preserva sua consistência. As transformações genéricas do espaço de estados que preservam a consistência física de um operador densidade possuem um estrutura bem definida por meio do teorema de representação de Kraus (PRESKILL, 1997; BREUER; PETRUCCIONE, 2002; WISEMAN; MILBURN, 2010).

Assim como os sistemas quânticos abertos, a área da medição foi amplamente desenvolvida, desde a década de 1970 (BRAGINSKY; KHALILI, 1995; DAVIES, 1976). A generalização dos procedimentos de medição ortogonais postulados por von Neumann e Lüders na década de 1950, por meio de medições baseadas no conceito matemático de medidas abstratas que tomam valores em um conjunto de operadores positivos, positive operator valued measure ou POVM, ampliou a compreensão dessa área na física quântica. Não é exagero dizer que a compreensão e interpretação dos modelos físicos relacionados aos processos de medição está relacionada a uma das maiores discussões realizadas pela física no século XX e que ainda persiste neste século (PRESKILL, 1997; BRAGINSKY; KHALILI, 1995; SAKURAI, 1994; COHEN-TANNOUDJI; DIU; LALOë, 1977; NIELSEN; CHUANG, 2000).

A medição de sistemas físicos é um processo dinâmico. O postulado de von Neumann-Lüders apresenta de forma idealizada o resultado final de um processo dinâmico de medição para modelos simplificados de sistemas quânticos. A incorporação da dinâmica de medição como um processo contínuo e não apenas como um acontecimento discreto, somente é possível por meio da abordagem sistemática dos sistemas quânticos abertos (BRAGINSKY; KHALILI, 1995; BREUER; PETRUCCIONE, 2002; JACOBS, 
2014; WISEMAN; MILBURN, 2010).

Nos modelos de experimentos de medição realísticos implementados na teoria de sistemas quânticos abertos, conclui-se que o papel do acoplamento via emaranhamento entre o sistema e o aparato de medição desempenha um papel fundamental. Conclui-se, então, que a decoerência exerce papel fundamental na modelagem dinâmica da medição. A partir das equações de evolução escotásticas é possível entender a decoerência como um processo de medição realizado pelo ambiente no sistema de interesse, ao qual não temos acesso e, portanto, não podemos tomar conhecimento dos dados medidos (BRUN, 2001; JACOBS; STECK, 2006; BREUER; PETRUCCIONE, 2002; WISEMAN; MILBURN, 2010).

A partir dos desenvolvimentos da teoria de medições contínuas, pôde ser obtida uma equação para filtrar o ruído de uma sequência de dados referente a uma variável dinâmica quântica. Essa equação desempenha o papel que classicamente é desempenhado pelo filtro de Kalman-Bucy e pela equação de Kushner-Stratonovich (WISEMAN; MILBURN, 2010; CARMICHAEL, 1991; CARMICHAEL, 1990; JACOBS, 2014; JACOBS; STECK, 2006; GARDINER; ZOLLER, 2004). A equação de filtragem quântica, quantum filtering equation, foi obtida originalmente na literatura por Belavkin (BELAVKIN, 1985) apud (JACOBS; STECK, 2006). Interessante notar que, em princípio, Belavkin partiu do filtro de Kalman para obter a equação dinâmica de evolução para a estimativa de um observável quântico, vide (BELAVKIN, 1985, p. 319). Assim, seria adequada a denominação do resultado por filtro de Kalman quântico ou por filtro de Kalman-Belavkin ou, ainda, filtro de Kalman-Bucy-Belavkin. ${ }^{8}$ Uma equação de filtragem para aplicação na dinâmica de sistemas na óptica quântica foi desenvolvida de forma independente em (CARMICHAEL, 1991; CARMICHAEL, 1990). O desenvolvimento do modelo pode ser realizado a partir da perspectiva de Heisenberg ou da perspectiva de Schrödinger, a escolha leva a dificuldades de ordem distinta. Por utilizar apenas cálculo estocástico clássico, o desenvolvimento de uma equação mestra, incorporando elementos estocásticos decorrentes do ruído de natureza quântica causado por um processo de medição contínuo, é de acesso mais simples (JACOBS; STECK, 2006; JACOBS, 2014; WISEMAN; MILBURN, 2010).

O tratamento formal dos fenômenos estocásticos em dinâmica quântica é realizado por meio da teoria de probabilidade quântica e do cálculo estocástico quântico, desenvolvidos a partir dos anos 1980 (HUDSON; PARTHASARATHY, 1984; PARTHASARATHY, 1992; BELAVKIN, 1983b; BELAVKIN, 1983a; BOUTEN; HANDEL; JAMES, 2006b; BOUTEN; HANDEL; JAMES, 2006a). Essas teorias desenvolvem as implicações lógicas relacionadas à não comutatividade dos operadores que representam as grandezas obser-

8 Acreditamos uma das duas últimas formas seria mais adequada, pela menção explícita a Belavkin, uma vez que a obtenção de estimadores de estados em sistemas dinâmicos quânticos não é trivial. 
váveis. A teoria de probabilidade quântica possui como caso particular a teoria de probabilidade clássica, decorrente dos axiomas de Kolmogorov. Essa particularização ocorre quando a álgebra de operadores auto adjuntos é do tipo QND, ou seja, é comutativa, o que implica que as medições realizáveis em um sistema quântico a partir de operadores dessa álgebra são compatíveis (BOUTEN; HANDEL; JAMES, 2006b; BOUTEN; HANDEL; JAMES, 2006a). As equações de evolução dos sistemas dinâmicos quânticos estocásticos podem ser encontradas sob várias formas, como equações de Schrödinger estocásticas, como equações mestras estocásticas ou como equações diferenciais estocásticas quânticas - também conhecidas como equações de Langevin quânticas (WISEMAN; MILBURN, 2010; GARDINER; ZOLLER, 2004; JACOBS; STECK, 2006; JACOBS, 2014; MABUCHI, 2014; DOHERTY et al., 2000).

A análise das equações quânticas estocásticas do tipo equação estocástica de Schrödinger e do tipo equação mestra estocástica mostra que se trata de equações não lineares. Antes, disso, devemos notar que o processo de medição de um observável $A$ possui como efeito dinâmico uma transformação no espaço de estados que é equivalente a uma transformação causada por um Hamiltoniano proporcional ao observável, ou seja, $H_{M_{A}}=f(A)$ (JACOBS; STECK, 2006; JACOBS, 2014). E, aparentemente, a incorporação dos efeitos dinâmicos da medição torna a equação de evolução não linear, com termos quadráticos no operador do observável $A$. Em conclusão, os processos de medição em sistemas dinâmicos quânticos interferem de forma essencial na evolução do sistema e esses efeitos devem ser estudados, com o objetivo de se atingir uma melhor compreensão dos sistemas dinâmicos quânticos com medição. A partir de uma boa compreensão desses sistemas dinâmicos, estará definida uma base para a abordagem do controle de sistemas quânticos em malha fechada.

\subsection{Propostas de Hardware}

No contexto da engenharia elétrica é importante mencionar a área de pesquisa em circuitos quânticos. Sob condições de trabalho em que o ruído térmico se torna menor que a energia dos fotons de frequências características de um circuito elétrico, seu comportamento passa a ser determinado quanticamente. Além de serem uma importante área de pesquisa em nanotecnologia, com grande potencial de aplicações na engenharia elétrica, os circuitos quânticos são também aplicáveis no desenvolvimento de uma arquitetura de computação quântica (BLAIS et al., 2004; GIRVIN, 2014; CLERK, 2014). Ressaltamos também que o regime quântico impõe limites fundamentais à amplificação de sinais (CLERK et al., 2010). Além dos circuitos, são igualmente importantes as máquinas quânticas, dispositivos mecânicos que também trabalham em regime quântico e possuem vasto potencial de aplicação na engenharia, pode-se consultar, por exemplo, (DEVORET et al., 2014). 
Igualmente importantes, tanto no desenvolvimento teórico das ferramentas analíticas aplicáveis a sistemas quânticos abertos e em medição contínua nos regimes de trabalho típicos para aplicação em computação quântica, quanto no desenvolvimento tecnológico de dispositivos para compor uma plataforma de computação quântica, são os sistemas da óptica quântica. A compreensão da linguagem da óptica quântica é importante para facilitar a compreensão de seus desenvolvimentos teóricos (WALLS; MILBURN, 2008; SCULLY; ZUBAIRY, 1997; WISEMAN; MILBURN, 2010). O primeiro sistema de controle quântico operando em malha fechada que temos conhecimento foi desenvolvido nessa área (WISEMAN; MILBURN, 1993; WISEMAN; MILBURN, 1994; WISEMAN, 1994; WISEMAN, 1995). Lembramos também que os Hamiltonianos construídos na plataforma de circuitos em QED possuem a mesma estrutura dos Hamiltonianos típicos da óptica quântica, em consequência, os conceitos da óptica quântica são utilizados para descrever e analisar os circuitos quânticos (BLAIS et al., 2004).

Há inumeras propostas de dispositivos de hardware e de arquiteturas para computação quântica, algumas até mesmo heterodoxas. Uma documentação desses dispositivos, de suas formas de operação, uma comparação entre seus parâmetros físicos e figuras de mérito e uma análise de sua aplicabilidade em plataformas de hardware para computação quântica poderia, em nossa opinião, originar um trabalho inteiro. 


\section{Controle de Sistemas Quânticos em Malha}

\section{Aberta}

Os domínios da física quântica estabelecem três diferenças fundamentais que devem ser devidamente tratadas pelas teorias de controle que pretendem avançar nesse campo de atuação:

- A composição de sistemas se dá pelo produto das partes e não por sua soma. É esse elemento que introduz correlações entre as variáveis dinâmicas distintas mesmo que essas variáveis sejam relativas a partes distintas do sistema global. ${ }^{1}$

- O espaço de Hilbert associado a diversos sistemas quânticos aparentemente simples, por exemplo, a dinâmica de uma partícula livre em uma dimensão ou a dinâmica de um oscilador harmônico, possui dimensão infinita. Mesmo esses dois exemplos diferem entre si. No caso do oscilador harmônico quântico, a energia somente pode assumir valores em um conjunto discreto, ao contrário da dinâmica da partícula livre, cuja energia é livre para variar continuamente. Como o espectro de operadores auto adjuntos em dimensão infinita pode ser tanto discreto quanto contínuo, alguns observáveis em dimensão infinita podem modelar experimentos de medição com resultados que podem variar no contínuo. Outra diferença importante é que alguns operadores auto adjuntos em dimensão infinita podem ser não limitados, ou seja, $\left\|A x_{n}\right\|$ pode ser infinita mesmo que $x_{n}$ tenha sempre norma unitária. A manipulação de operadores não limitados exige um cuidado especial com a definição de seu domínio de atuação. Para alguns casos, esse domínio pode até mesmo não ser denso no espaço de Hilbert. ${ }^{2}$

- A medição de um sistema quântico apresenta uma natureza fundamentalmente aleatória e a estrutura matemática subjacente aos modelos é do tipo não comutativa o que inviabiliza a aplicação da lógica booleana aos sistemas quânticos. ${ }^{3}$

1 É essa parte da estrutura da física quântica que está relacionada aos fenômenos aparentemente não locais que desafiam a teoria da relatividade. Uma discussão pormenorizada do paradoxo de EinsteinPodolski-Rosen e das desigualdades de Bell que tornaram explícitas as diferenças existentes entre a visão causal-relativística da realidade, expressa por Einstein, Podolski e Rosen, e as previsões da física quântica pode ser encontrada em (BERKOVITZ, 2014). As desigualdades de Bell são discutidas em muitos textos de física quântica, por exemplo, (NIELSEN; CHUANG, 2000; SAKURAI, 1994).

2 Os físicos, no entanto, costumam argumentar que a natureza não admite sistema que possuem energia infinita, portanto, os exemplos que fazem sentido físico não apresentam, ou não deveriam apresentar esses problemas. Na realidade, na física é comum encontrar abusos de notação. A notação de Dirac para sistemas quânticos introduzida no capítulo 1, por exemplo, só foi formalizada matematicamente anos depois que foi definida e amplamente utilizada na física.

3 A lógica quântica, como teoria de manipulação de projeções ortogonais em espaços de Hilbert, foi fundada nesses termos em 1936 por Birkhoff e von Neumann (BIRKHOFF; NEUMANN, 1936). Uma 
A física quântica estabeleceu nos domínios da física a quebra de paradigma anterior, que se costuma denominar atualmente "física clássica". Nesse contexto anterior das ciências naturais, conforme já se mencionou no primeiro capítulo, o determinismo era tomado como fundamento de tudo e também como modelo de perfeição. As ciências naturais se encontravam em uma corrida incessante por leis matemáticas perfeitas.

As incertezas, que sempre fizeram parte do trabalho dos experimentalistas, eram tomadas como imperfeições tecnológicas e não como princípios fundamentais. Acreditavase que o avanço da ciência permitia o avanço da tecnologia, que por sua vez levaria a um refinamento das teorias científicas. Um ciclo virtuoso que levaria o conhecimento científico a se aproximar cada vez mais da perfeição. A euforia da humanidade era tão grande com relação a essa perpectiva de avanço sem fim, que houve, no século XIX e início do século XX, uma tentativa de transpor os métodos científicos para fora dos limites das ciências naturais.

A física quântica, com sua interpretação fundamentalmente probabilística da natureza destruiu o sonho científico do determinismo. Os postulados da medição de um sistema quântico, já descritos no capítulo 1, estabeleceram que os resultados de um processo de medição têm uma natureza inafastavelmente aletória. Além disso, a não comutatividade dos observáveis estabelecia também um limite para a quantidade de informação que se poderia obter de um sistema físico.

No entanto, conforme se pretende expor nos próximos capítulos, ainda que introduza diferenças fundamentais na forma de se modelar um sistema físico, a teoria da medição quântica contínua estabele um modelo matemático de natureza estocástica que pode, inicialmente, ser tratado usando os métodos da teoria de controle clássica desenvolvidos para sistemas estocásticos.

Neste capítulo serão abordados alguns critérios de controlabilidade para sistemas quânticos fechados. A análise será iniciada pelo tratamento do caso em que a dimensão do sistema é finita. Em seguida, o problema da controlabilidade em dimensão infinita será introduzido, assim como as diversas dificuldades técnicas que devem ser contornadas para se chegar a uma solução. Ao final, será discutido um paralelo entre a controlabilidade em dimensão finita e infinita, utilizando vetores cíclicos. O ponto de partida será a forma canônica de Brunovski para sistemas controláveis. Em dimensão infinita, serão analisados alguns teoremas que estabelecem a existência de vetores cíclicos para conjuntos completos de observáveis que comutam (PRUGOVECKI, 1981). Será discutido se a existência de

\footnotetext{
introdução aos fundamentos matemáticos para a compreensão da teoria de reticulados (lattices), utilizada para descrever a lógica quântica, pode ser encontrada em (WILCE, 2012), em particular no anexo que trata de relações de ordenação. Interessante notar também que, como os axiomas de Kolmogorov, que definem a probabilidade clássica, resultam em uma teoria de probabilidades que é construída a partir da lógica booleana de operações com conjuntos, esta teoria não se adequa aos modelos quânticos (BOUTEN; HANDEL; JAMES, 2006b). A diferença fundamental se encontra na probabilidade conjunta ou simultânea de dois eventos.
} 
vetores cíclicos e de uma álgebra de operadores completa pode ser um sinal indicativo de controlabilidade em dimensão infinita. Pelo menos para protótipos de sistemas quânticos para aplicações de medição QND ou BAE, quantum non-demolition measurement ou back action evading measurement, pois tais sistemas são caracterizados por álgebras dinâmicas e de medição compostas de operadores auto adjuntos que comutam entre si.

As análises indicadas acima são realizadas na álgebra dos campos de vetores no caso de sistemas clássicos, ou na álgebra dos operadores auto adjuntos no caso dos sistemas quânticos. Será apresentada uma análise realizada no grupo de transformações unitárias do espaço de Hilbert $\ell^{2}$, por meio da qual será demonstrada a controlabilidade de um exemplo específico de sistema quântico em dimensão infinita (KARWOWSKI; VILELAMENDES, 2004; VILELA-MENDES, 2009; VILELA-MENDES; MANIKO, 2011).

Serão também analisados dois artigos importantes na área de controle quântico (HUANG; TARN, 1983) e (WU; TARN; LI, 2006). O primeiro é um dos trabalhos pioneiros na área, publicado em 1983. O segundo é uma generalização do primeiro, uma sequência já apontada originalmente pelos autores. A partir da apresentação e discussão dos resultados do segundo artigo, que estabelece condições para um sistema quântico ser controlável em uma variedade integral que tenha possivelmente dimensão infinita, será apontada uma analogia com sistemas dinâmicos que apresentam caos determinístico.

Os aspectos teóricos apresentados neste capítulo são baseados em sua maior parte na monografia (D'ALESSANDRO, 2008), quando estiverem no âmbito da dimensão finita, pode-se consultar também, nesse caso, (LISBOA, 2015).

\subsection{Sistemas de controle lineares}

Apresentaremos nesta seção algumas definições e resultados da teoria de controle clássica para sistemas lineares. O objetivo é apresentar a forma canônica de Brunovski para um sistema controlável. Alguns conceitos aqui dispostos serão resgatados ao final do capítulo para realizar uma analogia com os sistemas de controle quânticos de dimensão infinita. Seguimos as lições de Levine (LEVINE, 2009, p. 87 e ss.)

Definição 1 (Equivalência entre sistemas de controle). Dois sistemas dinâmicos $\dot{x}=$ $A x+B u$ e $\dot{z}=F z+G v$ são equivalentes por uma mudança de coordenadas linear e por realimentação não degenerada, equivalência SLF da sigla em inglês, se existem duas matrizes inversíveis, $M$ e $L$, e uma matriz $K$ tais que, se $x$ e $u$ satisfazem $\dot{x}=A x+B u$ e se $z=M x$ e $v=K x+L u$, então, $z$ e $v$ satisfazem $\dot{z}=F z+G v$.

A matriz $M$ representa a mudança de coordenadas, enquanto as matrizes $K$ e $L$ representam a realimentação. Da inversibilidade de $M$ e de $L$ conclui-se que dois sistemas SLF equivalentes possuem espaços de estados com a mesma dimensão e o mesmo número 
de entradas. Como a relação depende apenas das matrizes que definem o sistema, pode-se dizer que os pares $(A, B)$ e $(F, G)$ são SLF equivalentes. A definição 1 acima induz uma relação de equivalência entre os sistemas dinâmicos lineares.

No caso de sistemas com apenas uma entrada, a matriz $B$ reduz-se a um vetor, a ser denominado $b$ e o sistema fica denotado por $\dot{x}=A x+b u$, em que u é um escalar. A matriz de controlabilidade desse sistema será portanto:

$$
\mathcal{C}=\left(b|A b| \ldots \mid A^{n-1} b\right)
$$

Se o sistema for controlável, a matriz $\mathcal{C}$ terá posto $n$. Como, para um sistema de apenas uma entrada, a matriz de controlabilidade tem apenas exatamente $n$ colunas, elas são linearmente independentes, o que implica que os vetores $\left\{b, A b, \ldots, A^{n-1} b\right\}$ formam uma base para o espaço de estados $\mathbb{R}^{n}$.

Um sistema de controle linear com entrada simples pode, então, assumir uma forma particularmente simples, caso seja controlável, de tal forma que a base de $\mathbb{R}^{n}$ gerada pelo vetor cíclico $b$ seja a base canônica de do espaço.

Definição 2 (Forma canônica controlável). A forma canônica de um sistema de controle linear controlável com uma entrada, do tipo $\dot{z}=F z+G v$, é dada pelo seguinte par de matrizes:

$$
F=\left(\begin{array}{ccccc}
0 & 1 & 0 & \cdots & 0 \\
0 & 0 & 1 & \cdots & 0 \\
\vdots & & \ddots & \ddots & \\
0 & 0 & 0 & \cdots & 1 \\
0 & 0 & 0 & \cdots & 0
\end{array}\right), \quad g=\left(\begin{array}{c}
0 \\
0 \\
\vdots \\
0 \\
1
\end{array}\right)
$$

O resultado a seguir justifica a denominação forma canônica.

Teorema 3 (Universalidade da forma canônica controlável). Todo sistema de controle linear de uma entrada controlável, $\dot{x}=A x+b u$, é SLF equivalente a um sistema canônico do tipo 3.2, da forma $\dot{z}=F z+$ gv, com a mesma dimensão $n$.

Demonstração. A prova desse teorema, com a construção das matrizes da transformação pode ser encontrada em Levine (LEVINE, 2009, p. 91).

Finalmente, no caso em que temos múltiplas entradas de controle, a forma canônica controlável mantém a mesma estrutura do par 3.2 em cada um dos subespaços cíclicos gerados pelas colunas de $B$. A forma canônica geral é construída, portanto, em blocos. Assim, vale o teorema de Brunovski. 
Teorema 4 (Forma canônica controlável geral). Todo sistema de controle linear de uma entrada controlável, $\dot{x}=A x+b u$, é SLF equivalente a um sistema canônico do tipo 3.2, da forma $\dot{z}=F z+$ gv com a mesma dimensão $n$.

Demonstração. A prova desse teorema, com a construção das matrizes da transformação, pode ser encontrada, por exemplo, em Kailath (KAILATH, 1980) apud Levine (LEVINE, 2009, p. 92).

\subsection{Controlabilidade de sistemas quânticos de dimensão finita}

Dado o sistema quântico em dimensão finita:

$$
i \hbar \frac{d}{d t}|\psi(t)\rangle=H(u(t))|\psi(t)\rangle
$$

cuja dinâmica está sujeita ao vetor de controle $u(t)$, e dados o estado inicial, $\left|\psi_{0}\right\rangle \in \mathcal{E}=\mathbb{C}^{n}$ e o estado final, ou estado objetivo, $\left|\psi_{f}\right\rangle \mathcal{E}$, é necessário investigar se, $\forall\left|\psi_{0}\right\rangle \in \mathcal{E}$ e $\forall\left|\psi_{f}\right\rangle \in$ $\mathcal{E}, \exists \hat{u}(t)$ definida em $[0, T)$ tal que $|\psi(T)\rangle=\left|\psi_{f}\right\rangle$, ou seja, se o sistema é controlável. E, para sistemas quânticos controláveis, como escolher $u^{*}(t) \in\{\hat{u}(t)\}$, de forma a satisfazer determinados critérios de otimalidade ou restrições de controle impostas pelo sistema físico.

Mais especificamente, o interesse maior recai sobre sistemas de controle bilineares:

$$
i \hbar \frac{d}{d t}|\psi\rangle=\left(H_{0}+\sum_{k} u_{k}(t) H_{k}\right)|\psi\rangle
$$

em que $H_{0}$ é hamiltoniano livre, chamado termo de drift, $\left\{H_{k}\right\}$ são os hamiltonianos de controle, e $u(t)$ são funções definidas em intervalos de $\Re$, tomando valores em $\Re$ ou subconjuntos de $\Re$, como por exemplo $\{0,1\}$.

Em função da natureza dos sistemas quânticos, que restringe os estados quânticos $|\psi\rangle \in \mathbb{C}^{n}$ de forma a que se satisfaça a condição de normalização $\||\psi\rangle \|=1$, trata-se de um sistema de controle linear definido sobre a esfera unitária em $\mathbb{C}^{n}$ equivalente a $S^{2 n-1}$, ou seja, sobre uma variedade diferenciável não globalmente difeomórfica a um espaço vetorial simples. Geometricamente os hamiltonianos citados são equivalente a campos de vetores definidos no espaço tangente à esfera.

Os métodos da geometria diferencial e da teoria de controle geométrico são os mais adequados e naturais para as investigações sobre a controlabilidade dos sistemas quânticos indicados assim como para a definição de estratégias de controle, como controle ótimo e controle robusto, por exemplo. 
A equação 3.3 induz um grupo de transformação a um parâmetro $\left\{X_{t}\right\}$ sobre $\mathbb{C}^{n}$ que satisfaz:

$$
i \hbar \frac{d}{d t} X_{t}=H(u(t)) X_{t}
$$

para o qual $X_{0}=I$, a matriz identidade.

Para todo $t \in \mathbb{R}$ e para toda função de controle $u, X_{t}$ é uma matriz unitária com determinante igual a 1 o que faz com que $\left\{X_{t}\right\}_{t \in \mathbb{R}} \subseteq S U(n)$.

A evolução do sistema quântico pode ser visualizada como uma curva na esfera unitária, representando a dinâmica do estado quântico. Uma segunda possibilidade, é utilizar os grupos de simetria do espaço subjacente, no caso da esfera unitária, e representar a evolução por uma curva no grupo de transformação definido em 3.5. No caso em estudo, uma curva em $S U(n)$.

Em função da equivalência local entre $S U(2)$, o grupo de spin de duas dimensões, e $S O(3)$, o grupo ortogonal especial do espaço tridimensional, existe a representação pictórica de um sistema quântico de duas dimensões como um ponto na esfera unitária $S^{2}$. Essa analogia geométrica do espaço de estados subjacente ao sistema tratado é chamada esfera de bloch.

Exemplo 5 (Esfera de Bloch). O estado de um sistema quântico de duas dimensões pode ser geometricamente representado pela esfera $S^{2}$, equivalente ao espaço de configurações de um corpo rígido com um ponto fixo.

A dinâmica do sistema clássico que modela as rotações de um corpo rígido em torno de um ponto fixo pode ser comparada com a dinâmica da evolução do spin de uma partícula subatômica (NEUMAIER; WESTRA, 2008). Os aspectos mais relevantes do problema de controle para a configuração do corpo rígido podem ser transpostos para o caso quântico equivalente. O grande interesse nesse exemplo está em que, para o sistema quântico em malha aberta, os resultados da teoria de controle de sistemas clássicos poderão ser aplicados, uma vez que localmente existe uma equivalência entre os espaços de estados dos dois sistemas. No entanto, os espaços $S U(2)$ e $S O(3)$ não são globalmente difeomórficos. A partir do momento em que os aspectos globais do espaço subjacente se tornarem suficientemente relevantes ao problema, a analogia apresentada acima pode deixar de ser verdadeira.

Há ainda uma terceira possibilidade de visualização geométrica da dinâmica dos sistemas quânticos, a evolução dinâmica de operadores densidade que, de forma alternativa aos vetores de estado, modelam a evolução de sistemas quânticos. Nesse caso, a evolução do sistema se dá no espaço de matrizes quadradas que representam operadores positivos com traço igual a 1 . 
As abstrações apresentadas levam à definição de três noções distintas de controlabilidade, conforme definições apresentadas a seguir (D'ALESSANDRO, 2008).

Definição 6 (Controlabilidade por Estados Puros). Se em um sistema de controle quântico sempre existe um controle $u(t)$ capaz de levar o estado inicial $\left|\psi_{0}\right\rangle$ ao estado objetivo $\left|\psi_{f}\right\rangle$ quaisquer que sejam estes estados então o sistema é dito controlável em nível de estados puros.

Definição 7 (Controlabilidade por Estados Equivalentes). Se em um sistema de controle quântico sempre existe um controle $u(t)$ capaz de levar o estado inicial $\left|\psi_{0}\right\rangle$ ao estado $\left|\psi_{f}^{*}\right\rangle$, equivalente ao estado objetivo $\left|\psi_{f}\right\rangle$ por um fator de fase $e^{i \phi}$ arbitrário, quaisquer que sejam os estados inicial e final, então, o sistema é dito controlável em nível de estados equivalentes.

Definição 8 (Controlabilidade por Matriz Densidade). Se em um sistema de controle quântico sempre existe um controle $u(t)$ capaz de levar o estado inicial $\rho_{0}$ ao estado objetivo $\rho_{f}$ quaisquer que sejam os estados inicial e final, desde que sejam unitariamente equivalentes, então, o sistema é dito controlável em nível de matriz densidade.

A partir da teoria de controle geométrico clássica pode-se chegar ao seguinte resultado (D'ALESSANDRO, 2008).

Teorema 9 (Álgebra de Lie Dinâmica). O conjunto de estados alcançáveis por um sistema dinâmico quântico é dado pelo grupo de Lie conexo, $\mathcal{R}$, associado à álgebra $\mathcal{L}$ gerada por $\operatorname{span}_{u}\{-i H(u)\}$, ou seja,

$$
\mathcal{R}=e^{\mathcal{L}}
$$

Demonstração. A prova desse teorema pode ser encontrada em (D'ALESSANDRO, 2008).

Para o caso da Controlabilidade por Estados Puros é possível obter o seguinte resultado:

Teorema 10 (Condições para Controlabilidade por Estados Puros). Um sistema quântico é Controlável por Estados Puros se, e somente se, sua correspondente Álgebra de Lie $\mathcal{L}$ satisfizer uma das seguintes condições:

1. $\mathcal{L}=s u(n)$

2. $\mathcal{L}=u(n)$

3. $\mathcal{L}$ é uma álgebra conjugada a $\operatorname{sp}\left(\frac{n}{2}\right)$ 
4. $\mathcal{L}=\operatorname{span}\left\{i \mathbf{1}_{n}\right\} \oplus \tilde{\mathcal{L}}$, em que $\tilde{\mathcal{L}}$ é uma álgebra conjugada a $\operatorname{sp}\left(\frac{n}{2}\right)$

Demonstração. A prova desse teorema pode ser encontrada em (D'ALESSANDRO, 2008).

Em vista do teorema abaixo, o tratamento do caso de Controlabilidade por Estados Equivalentes é desnecessário.

Teorema 11 (Controlabilidade por Estados Equivalentes). Um sistema quântico é controlável por estados puros se, e somente se, é controlável por estados equivalentes.

Demonstração. A prova desse teorema pode ser encontrada em (D'ALESSANDRO, 2008).

Para o caso da Controlabilidade por Matriz Densidade é possível obter o seguinte resultado:

Teorema 12 (Condições para Controlabilidade por Matriz Densidade). Um sistema quântico é Controlável por Matriz Densidade se, e somente se, sua correspondente Álgebra de Lie $\mathcal{L}$ satisfizer uma das seguintes condições:

1. $\mathcal{L}=s u(n)$

2. $\mathcal{L}=u(n)$

Demonstração. A prova desse teorema pode ser encontrada em (D'ALESSANDRO, 2008).

Numericamente, os modelos quânticos podem ter sua dinâmica simulada diretamente. O fator de dificuldade a ser superado nesse caso é a manipulação direta de matrizes complexas ou a conversão do espaço vetorial complexo subjacente, $\mathbb{C}^{n}$, em um espaço vetorial real, $\mathbb{R}^{2 n}$, vide (D'ALESSANDRO, 2008, pp. 160-161) para verificar uma transformação que realiza essa conversão. Geometricamente, o estado do sistema também está restrito aos vetores complexos de norma unitária.

A aplicação de técnicas de controle clássicas, mesmo técnicas de malha fechada, pode ser realizada por meio de um modelo virtual da planta quântica. Desse modelo virtual, é possível extrair diretamente uma estimativa do estado do sistema quântico e utilizá-la para calcular o controle $u(t)$ que será efetivamente utilizado para dirigir o sistema quântico real.

Na prática, o uso de sistemas de controle em malha aberta é limitado pelo fato de haver incertezas relacionadas a diversos aspectos do modelo matemático, e mesmo 
da teoria física que lhe dá sustentação. Pode-se destacar a incerteza do estado inicial a ser dirigido, a incerteza dos parâmetros físicos do modelo, a incerteza do modelo (por exemplo, relacionada a simplificações de um modelo mais completo ou relacionadas aos efeitos da decoerência, que foram ignorados) e também a incerteza relacionada aos campos de vetores utilizados para controlar o sistema. Todos esses fatores levarão a uma incerteza no estado final, ou estado objetivo.

As análises teóricas da área da computação quântica permitem o projeto de sistemas tolerantes a falhas, ou seja, sistemas computacionais robustos capazes de eliminar o efeito de pequenas imperfeições no sistema de hardware, garantindo um resultado final de processamento quântico confiável (PRESKILL, 1997). Para uma determinada arquitetura de hardware, foi recentemente apresentado um limite superior de $3 \%$ para as imperfeições do hardware que compõe um determinado sistema (KNILL, 2005). Dentro desse limite o processamento de informação quântica ocorre sem ser afetado por eventuais falhas de hardware.

Assim, dado um modelo matemático para um sistema físico implementando um ou mais qubits, é possível determinar numericamente os limites máximos para as incertezas do modelo de forma a permitir que o sistema seja utilizável para a construção de hardware quântico, controlado em malha aberta.

\subsection{Controlabilidade de Sistemas de dimensão infinita}

A seguir apresentaremos duas definições importantes no tratamento de sistemas quânticos de dimensão infinita, a definição de conjunto completo de operadores e a definição de vetor cíclico.

Heuristicamente, de acordo com a formulação original (DIRAC, 1930) apud (PRUGOVECKI, 1981, p. 311), um conjunto completo de observáveis $\left\{A_{1}, \ldots, A_{n}\right\}$ é uma família de operadores auto adjuntos no espaço de estados de um sistema quântico, portanto, um espaço de Hilbert, que possuem espectro discreto, representado para cada um dos operadores do conjunto por $S^{A_{k}}=\left\{\lambda_{k}^{(1)}, \lambda_{k}^{(2)}, \ldots\right\}$, e que satisfazem às seguintes propriedades.

1. A cada elemento $\left(\lambda_{1}, \ldots, \lambda_{n}\right) \in S^{A_{1}} \times S^{A_{2}} \times \ldots \times S^{A_{n}} \equiv S$ pode ser associado um vetor, denotado $\left|\psi_{\lambda_{1}, \ldots, \lambda_{n}}\right\rangle$ pertencente ao domínio de definição comum dos operadores $A_{1}, \ldots, A_{n}$, satisfazendo:

$$
A_{k}\left|\psi_{\lambda_{1}, \ldots, \lambda_{n}}\right\rangle=\lambda_{k}\left|\psi_{\lambda_{1}, \ldots, \lambda_{n}}\right\rangle
$$

2. O conjunto $\left\{\left|\psi_{\lambda_{1}, \ldots, \lambda_{n}}\right\rangle\right\}$ para $\left(\lambda_{1}, \ldots, \lambda_{n}\right) \in S$ é uma base ortonormal do espaço de Hilbert ${ }^{4}$.

$\overline{4}$ Ao lidar com sistemas de multiplas partículas idênticas, que seguem a estatística de Bose-Einstein 
Se um operador perfaz, por si só, um conjunto completo $\{A\}$, diz-se que seu espectro é simples.

Como $\left\{\left|\psi_{\lambda_{1}, \ldots, \lambda_{n}}\right\rangle\right\}$ é uma base para o espaço de Hilbert, os operadores lineares auto adjuntos do conjunto $\left\{A_{i}\right\}$ possuem um domínio comum denso. E nesse domínio comum os operadores comutam entre si.

Como:

$$
\begin{aligned}
A_{i} A_{j}\left|\psi_{\lambda_{1}, \ldots, \lambda_{n}}\right\rangle & =\lambda_{i} \lambda_{j}\left|\psi_{\lambda_{1}, \ldots, \lambda_{n}}\right\rangle \\
A_{j} A_{i}\left|\psi_{\lambda_{1}, \ldots, \lambda_{n}}\right\rangle & =\lambda_{j} \lambda_{i}\left|\psi_{\lambda_{1}, \ldots, \lambda_{n}}\right\rangle
\end{aligned}
$$

Pela comutatividade dos escalares $\lambda_{i}$ e $\lambda_{j}$, conclui-se que $\left[A_{i}, A_{j}\right]=0$.

Importante notar que a formulação heurística de Dirac, pode ser formalizada corretamente para os operadores lineares auto adjuntos que possuem espectro discreto apenas. Para operadores que possuem também espectro contínuo, é necessária uma generalização, apresentada a seguir.

Definição 13 (Conjunto Completo de Operadores). O conjunto de operadores auto adjuntos $\left\{A_{1}, \ldots, A_{n}\right\}$ vinculados a um espaço de Hilbert $\tilde{H}$, espaço de estados de um dado sistema quântico, constitui um conjunto completo de operadores em $H$, subespaço de $\tilde{H}$, se as três condições abaixo são satisfeitas.

1. Existe uma medida $\mu(B)$ nos conjuntos de Borel $B$ de $\mathbb{R}^{n}$ com suporte $S$ contido em $S^{A_{1}} \times S^{A_{2}} \times \ldots \times S^{A_{n}}$ em que $S^{A_{k}}$ é o espectro de cada um dos operadores $A_{k}$.

2. Existe uma transformação unitária $U$ entre o espaço $\tilde{H}$ e o espaço $L^{2}\left(\mathbb{R}^{n}, \mu\right)$ tal que os operadores

$$
A_{k}^{\prime}=U A_{k} U^{-1}
$$

se reduzem a operadores de multiplicação ${ }^{5}$

$$
\left(A_{k}^{\prime} \psi\right)(x)=x_{k} \psi(x)
$$

com domínio de definição

$$
\mathcal{D}_{A_{k}^{\prime}}=\left\{\psi: \int_{\mathbb{R}^{n}} x_{k}^{2}|\psi(x)|^{2} d \mu(x)<\infty, \psi \in L^{2}\left(\mathbb{R}^{n}, \mu\right)\right\}
$$

ou a estatística de Fermi-Dirac, nem todos os estados do conjunto ortonormal representam estados válidos para o sistema, mas somente aqueles estados pertencentes aos subespaços fechados formados pelos produtos tensoriais simétrico e antisimétrico respectivamente. Este é um axioma adicional da física quântica que afirma que partículas quânticas de mesma natureza são indistinguíveis entre si. Para uma discussão a esse respeito vide Prugovecki (PRUGOVECKI, 1981, p. 305-310 e 312-315 )

5 No tratamento de vetores em espaços de Hilbert de dimensão infinita representados por espaços de funções, a notação explícita utilizada a seguir é mais clara que a notação de Dirac, o que facilita a compreensão dos conceitos. Assim, quando considerado necessário, será feito este uso. 
3. $U$ é uma bijeção entre $H$ e o subespaço $\hat{L}^{2}\left(\mathbb{R}^{n}, \mu\right)$ de $L^{2}\left(\mathbb{R}^{n}, \mu\right)$ das funções de estado apropriadamente simetrizadas.

(PRUGOVECKI, 1981, p. 315), observa que ordinariamente e de forma tácita, os modelos matemáticos de sistemas quânticos assumem a existência de um conjunto completo de operadores $\left\{A_{1}, \ldots, A_{n}\right\}$, finito, o que poderia até mesmo ser tratado como um axioma da teoria. Como consequência dessa hipótese, toda função $F\left(A_{1}, \ldots, A_{n}\right)$ que mantenha invariante o subespaço $H$ definido pela base ortonormal é, nesse subespaço, um observável do sistema quântico ${ }^{6}$.

Definição 14 (Vetor Cíclico). O vetor $\psi_{0} \in H$ é um vetor cíclico em $\tilde{H} \subset H$ com relação a um conjunto de operadores $\left\{A_{1}, \ldots, A_{n}\right\}$, se as três condições a seguir são satisfeitas:

1.

$$
\psi_{0} \in \cap_{k} \mathcal{D}_{A_{k}} \equiv \mathcal{D}
$$

2. Para quaisquer números inteiros não negativos $k_{1}, \ldots, k_{n} \in\{0,1,2, \ldots\}$

$$
A_{1}^{k_{1}} \ldots A_{n}^{k_{n}} \psi_{0} \in \mathcal{D}
$$

3. O subespaço vetorial

$$
\mathcal{D}\left(\psi_{0} ; A_{1}, \ldots, A_{n}\right)=\left\{p\left(A_{1}, \ldots, A_{n}\right) \psi_{0}\right\}
$$

formado por todos os vetores da forma $p\left(A_{1}, \ldots, A_{n}\right) \psi_{0}$, correspondentes a todos os polinômios apropriadamente simetrizados $p$ em $A_{1}, \ldots, A_{n}$, é denso em $H$.

A seguir será apresentada uma importante relação entre os conceitos de conjunto completo de operadores e de vetor cíclico (PRUGOVECKI, 1981, p. 317).

Teorema 15 (Relação entre Vetores Cíclicos e Conjuntos de Operadores). O conjunto de operadores auto adjuntos $\left\{A_{1}, \ldots, A_{n}\right\}$ de um espaço de Hilbert separável $H$, com imagem $\tilde{H} \subset H$ é completo se, e somente se, existir um vetor $\psi_{0} \in H$, cíclico com relação ao conjunto de operadores $\left\{A_{i}\right\}$. Nesse caso, os operadores auto adjuntos comutarão entre si no subespaço vetorial de $H$ gerado pela aplicação de $\psi_{0}$ a todos os polinômios da forma $p\left(A_{1}, \ldots, A_{n}\right)$, devidamente simetrizados.

Demonstração. A prova deste teorema é bastante técnica e envolve a utilização não trivial de conceitos de teoria da medida e de análise funcional. Pode ser encontrada em (PRUGOVECKI, 1981, p. 317-324).

$\overline{6}$ Nem todos os operadores do conjunto completo $\left\{A_{1}, \ldots, A_{n}\right\}$ ou suas funções $F\left(A_{1}, \ldots, A_{n}\right)$ representam observáveis, quando o sistema quântico trata de partículas idênticas. No entanto, funções apropriadamente simetrizadas dos operadores representam observáveis no sistema, vide (PRUGOVECKI, 1981, p. 316). 
Mais interessante aos propósitos dessa análise é um exemplo não trivial da utilização desses conceitos.

Exemplo 16 (Estados Gaussianos). Seja $H$ o espaço de Hilbert $L^{2}(\mathbb{R})$ e $X$ o operador auto adjunto posição.

$$
(X \psi)(x)=x \psi(x)
$$

Assim, $\{X\}$ é um conjunto completo, portanto, $X$ possui espectro simples e, ainda, $\psi_{0}(x)=\exp \left(-x^{2}\right)$ é um vetor cíclico para o operador posição, uma vez que se pode mostrar que $\left\{p(x) \exp \left(-x^{2} / 2\right)\right\}$, em que $p(x)$ é um polinômio, é denso em $L^{2}(\mathbb{R})$.

Este exemplo demonstra também que as representações espectrais dos operadores não são únicas, uma vez que diferentes medidas $\mu$ conduzem a representações distintas, vide (PRUGOVECKI, 1981, p. 324).

O exemplo 16 acima é particularmente interessante para moldar a intuição nos casos em que o espaço de Hilbert possui dimensão infinita, uma vez que demonstra que o conjunto das distribuições gaussianas em $\mathbb{R}$ é um conjunto denso. Esse fato é também interessante no contexto dos sistemas quânticos abertos, no qual os modelos de sistemas podem ser expandidos para abranger processos de medição contínuos, pois os modelos matemáticos resultantes são estocásticos e as distribuições gaussianas são, portanto, fundamentais.

Para finalizar, destacamos o teorema abaixo, que estabelece a propriedade da maximalidade para os conjuntos completos de operadores auto adjuntos.

Teorema 17 (Maximalidade dos Conjuntos de Operadores). Se o operador limitado A, definido no espaço de Hilbert $\tilde{H}$, comuta com os operadores de um conjunto completo de operadores $\left\{A_{1}, \ldots, A_{n}\right\}$, então, $A=F\left(A_{1}, \ldots, A_{n}\right){ }^{7}$

Demonstração. A prova desse teorema pode ser encontrada em (PRUGOVECKI, 1981, p. 327).

Neste ponto, já é possível vislumbrar a analogia buscada entre as noções de controlabilidade existentes para espaços de dimensão finita e de dimensão infinita. O conceito usado para fazer a conexão é o de vetor cíclico, que existe independentemente da dimensão do espaço de estados e que pode ser usado em ambos os casos para investigar as

$\overline{7} \quad$ O operador $A$ na hipótese do teorema é tomado como limitado, no entanto, no caso de um operador não limitado cujo domínio contenha a base ortonormal definida pelos operadores auto adjuntos do conjunto completo, seria possível afirma que, pelo com relação a essa restrição de domínio, seria possível definir uma sequência de funções $F_{i}\left(A_{1}, \ldots, A_{n}\right)$ convergente a $A$ no subespaço gerado pela base ortonormal. Assim, o operador $A$, não limitado, poderia ser aproximado por uma função $F\left(A_{1}, \ldots, A_{n}\right)$ em uma parte de seu domínio. Este arrazoado é no entanto mera conjectura. 
características topológicas do conjunto alcançável de uma álgebra de operadores - ou de uma álgebra de campos de vetores, nomenclatura usual em dimensão finita.

Contudo, vale a pena resgatar mais alguns conceitos e resultados da análise funcional relacionados aos vetores cíclicos. Seguiremos desta vez a exposição de Reed e Simon (REED; SIMON, 1980, p. 226 e ss.). Nessa monografia, os autores buscam inspiração nos espaços vetoriais de dimensão finita para definir a classe de operadores que pode ser decomposta com apenas uma medida $\mu$ em todo domínio de definição das funções $\psi(x) \in H$.

Definição 18 (Operadores sem multiplicidade). Dizemos que um operador auto adjunto limitado $A$ é sem multiplicidade quando é unitariamente equivalente à multiplicação por $\lambda$ em $L^{2}(\mathbb{R}, d \mu)$ para alguma medida $\mu .^{8}$

Uma caracterização interessante dos operadores sem multiplicidade é dada pelas proposições equivalentes abaixo.

Teorema 19 (Caracterização de operadores sem multiplicidade). São condições equivalentes:

1. A é um operador sem multiplicidade.

2. A possui um vetor cíclico.

3. $\{B \mid A B=B A\}$ é uma álgebra abeliana.

Demonstração. A prova desse teorema é deixada como exercício em (REED; SIMON, 1980, p. 247, ex. 22).

A caracterização da álgebra abeliana de operadores auto adjuntos que comutam é realizada a partir das respectivas medidas.

Definição 20 (Medidas equivalentes). Duas medidas de Borel são equivalentes se, e somente se, possuem os mesmos conjuntos de medida nula. Uma classe de equivalência $[\mu]$ é chamada classe de medida.

A relação de equivalência acima é motivada pelo teorema de Radon-Nikodym da seguinte forma. Sejam dadas uma medida $d \mu$ em $\mathbb{R}$ e uma função mensurável positiva e não nula, a menos de um conjunto de medida nula em $\mu$, e tal que

$$
\int_{C}|F| d \mu<\infty, \quad \forall C \subset \mathbb{R} \text { compacto }
$$

8 No caso, $\lambda$ é a variável do espaço de funções, no exemplo 16 acima, a variável do espaço foi usualmente denotada por $x$. 
Então, $d \nu=F d \mu$ é uma medida de Borel e a aplicação

$$
U: L^{2}(\mathbb{R}, d \nu) \rightarrow L^{2}(\mathbb{R}, d \mu)
$$

dado por

$$
(U f)(\lambda)=\sqrt{F(\lambda)} f(\lambda)
$$

é unitária e sobrejetora, e

$$
\lambda(U f)=U(\lambda f)
$$

Assim, um operador com representação espectral em termos de $\mu$ pode ser também representado em termos de $d \nu$. Pelo teorema de Radon-Nikodym, $d \nu=F d \mu, \operatorname{com} F$ não nula, a menos de um conjunto de medida nula, se, e somente se, $\nu$ e $\mu$ possuem os mesmos conjuntos de medida nula.

A questão da unicidade da representação espectral fica sedimentada, então, com a seguinte proposição.

Proposição 21 (Unicidade da representação espectral). Sejam $\mu$ e $\nu$ medidas de Borel em $\mathbb{R}$ com suporte limitado, $A_{\mu}$ e $A_{\nu}$ dois operadores em $L^{2}(\mathbb{R}, d \mu)$ e $L^{2}(\mathbb{R}, d \nu)$ respectivamente dados por

$$
\begin{aligned}
& \left(A_{\mu} f\right)(\lambda)=\lambda f(\lambda) \quad e \\
& \left(A_{\nu} f\right)(\lambda)=\lambda f(\lambda)
\end{aligned}
$$

Nessas condições, $A_{\mu}$ e $A_{\nu}$ são unitariamente equivalentes se, e somente se, $\mu$ e $\nu$ forem medidas equivalentes.

Demonstração. Essa proposição é também apresentada sem prova em (REED; SIMON, 1980, p. 232).

Para operadores auto adjuntos que possuem multiplicidade em seu espectro, existe uma decomposição do espaço de Hilbert em subespaços invariantes nos quais a multiplicidade do operador é uniforme e dada por $m$. Em cada subespaço invariante o operador é equivalente a uma multiplicação por $\lambda$ em $L^{2}(\mathbb{R}, d \mu) \oplus L^{2}(\mathbb{R}, d \mu) \oplus \ldots \oplus L^{2}(\mathbb{R}, d \mu)$, ou seja, $L^{2}(\mathbb{R}, d \mu)$ por $m$ vezes. A analogia com os operadores hermitianos em $\mathbb{C}^{n}$ é estrita. maiores detalhes podem ser encontrados em (REED; SIMON, 1980, p. 233-234)

Após a exposição sobre vetores cíclicos realizada acima é possível conjecturar algumas possíveis conclusões. A classe de sistemas quânticos a partir da qual se podem construir experimentos de medição do tipo QND ou BAE, quantum non-demolition measurement ou back action evading measurement, é caracterizada por observáveis e Hamiltonianos que comutam. Portanto, a álgebra abeliana completa de operadores autoadjuntos que comutam pode propiciar uma estrutura adequada à abordagem de um problema de controle com essas características. A questão da medição em si somente será abordada 
no próximo capítulo, no entanto, as soluções dinâmicas podem ser analisadas com as ferramentas apresentadas acima. Inicialmente, é possível concluir que qualquer observável que seja apto a compor um sistema QND ou BAE é um elemento do conjunto completo de operadores autoadjuntos associado à álgebra dinâmica do sistema. Por outro lado, todos os Hamiltonianos compatíveis com a dinâmica livre do sistema e com o conjunto de observáveis utilizados para monitorar o sistema são elementos do mesmo conjunto de operadores. E também as subalgebras contidas no conjunto completo de observáveis podem ser utilizadas pera definir sistemas dinâmicos em subespaços fechados nos quais se poderia modelar um experimento QND ou BAE.

A seguir apresentamos um exemplo de um sistema de controle quântico em um espaço de estados de dimensão infinita obtido em (KARWOWSKI; VILELA-MENDES, 2004). Trata-se de um modelo matemático qualitativo de uma partícula com carga cujo movimento está restrito a um círculo, onde pode se movimentar livremente. O operador Hamiltoniano do sistema é dado por:

$$
H_{0}=-\frac{\partial^{2}}{\partial \phi^{2}}, \quad \phi \in[0,2 \pi)
$$

O espaço de estados é dado pelo espaço de Hilbert $L^{2}([0,2 \pi))$ e o domínio de trabalho é definido por:

$$
\mathcal{D}\left(H_{0}\right)=\left\{f \in \mathcal{C}^{2} \mid f(2 \pi)=f(0)\right\}
$$

Os autoestados do Hamiltoniano são dados pelo conjunto:

$$
\left\{|k\rangle=e^{i k \phi} \mid k \in \mathbb{Z}\right\}
$$

com autovalores correspondentes a $k^{2}$.

A interação do sistema com campos magnéticos ortogonais ao plano definido pelo círculo é dada pelos operadores unitários:

$$
\begin{aligned}
& U_{+}=e^{i \phi} \quad e \\
& U_{-}=e^{-i \phi}
\end{aligned}
$$

A ação dos operadores unitários $U_{+}$e $U_{-}$é dada por:

$$
\begin{aligned}
& U_{+}|k\rangle=|k+1\rangle \quad e \\
& U_{-}|k\rangle=|k-1\rangle
\end{aligned}
$$

Como o espaçamento entre os níveis de energia é não uniforme, é possível excitar as transições de estado de forma independente entre si e também programar pulsos para realizar rotações no grupo $U(2)$ que age no subespaço gerado por dois autoestados sucessivos (KARWOWSKI; VILELA-MENDES, 2004). 
Uma transformação entre os espaços de Hilbert $L^{2}([0,2 \pi))$ e $\ell^{2}(\mathbb{Z})$ é sempre possível, pois os espaços de Hilbert separáveis de mesma dimensão são sempre isomorfos entre si. O espaço das sequências de números reais, cuja série quadrática é somável, é o cenário natural para o estudo dos operadores de "shift" para a direita, $e_{k} \mapsto e_{k+1}$, e de "shift" para a esquerda, $e_{k} \mapsto e_{k-1}$, em que $e_{k}$ é a base canônica de $\ell^{2}(\mathbb{Z})$.

O principal resultado obtido em (KARWOWSKI; VILELA-MENDES, 2004) é expresso por:

Teorema 22 (Caracterização do grupo gerado por $\left\{U(2), U_{+}\right\}$). Para todo $a \in \ell^{2}(\mathbb{Z})$ não nulo, o conjunto definido pela ação do grupo gerado por $\left\{U(2), U_{+}\right\}$em a é denso em $a \in \ell^{2}(\mathbb{Z})$.

Demonstração. A prova desse teorema apresentada em (KARWOWSKI; VILELA-MENDES, 2004).

Assim, por meio de composição de elementos do grupo de transformações unitárias gerado no exemplo acima é possível aproximar, com precisão arbitrária, qualquer elemento do espaço. A semelhança entre o resultado acima e as exposições sobre vetores cíclicos realizadas tanto em dimensão finita quanto em dimensão infinita é tentadora, no entanto, é preciso lembrar que nessas exposições anteriores os vetores cíclicos estavam vinculados à álgebra de controle ${ }^{9}$, no caso de dimensão finita, e à álgebra de operadores auto adjuntos no espaço de estados. Em suma, um conceito está vinculado aos campos de vetores que geram o sistema dinâmico por meio de uma equação diferencial enquanto o outro está vinculado ao sistema dinâmico em si, ou seja, ao fluxo gerado pela equação diferencial. Assim, a relação entre eles, ainda que possa existir, não é necessariamente simples e direta.

É interessante acrescentar também que a mesma estrutura utilizada para demonstrar a controlabilidade para sistemas quânticos fechados, realizada acima, pode ser utilizada para demonstrar a controlabilidade para sistemas quânticos abertos (VILELAMENDES, 2009). Nesse outro caso, os operadores shift também desempenham papel fundamental.

O exemplo apresentado acima demonstra a importância das operações de shift na construção de sistemas dinâmicos que permitem a controlabilidade de estados. Utilizamos aqui o conceito de sistema dinâmico como grupo de auto transformações do espaço. Em dimensão finita, os sistemas de controle possuem em geral uma álgebra de controle também finita. É comum, no entanto, que o conjunto gerador tenha um número de elementos inferior à dimensão do espaço. É a composição dos elementos que leva à geração de uma dinâmica universal. ${ }^{10}$

$9 \quad$ Ao par $(A, b)$, mais especificamente

10 A composição de campos de vetores entre si por meio da operação de comutação gera novos elementos, linearmente independentes de seus geradores. 
Após essa introdução, na qual resgatamos alguns conceitos de sistemas dinâmicos lineares e de análise funcional aplicada à caracterização de conjuntos de operadores auto adjuntos, apresentaremos alguns dos resultados mais importantes encontrados na literatura existente sobre sistemas de controle quânticos em dimensão infinita. Iniciaremos pelos conceitos e resultados introduzidos por (HUANG; TARN, 1983).

Trata-se de uma iniciativa pioneira de incorporar a dinâmica de sistemas quânticos ao arcabouço teórico da teoria de controle. Não consiste em tarefa simples, uma vez que os operadores lineares que definem a dinâmica do sistema e que representam os observáveis do sistema podem ter espectro contínuo e também ser não limitados. Apesar de as teorias espectrais de operadores em espaços de dimensão finita e infinita serem semelhantes, os fundamentos matemáticos necessários à formalização da teoria espectral em dimensão infinita com operadores de espectro contínuo são muito maiores. ${ }^{11}$ Ainda, quando os operadores são não limitados, as restrições de domínio devem ser devidamente tratadas para evitar que a aplicação de um operador a um dado vetor seja indeterminada. Felizmente, a classe de operadores de maior interesse na modelagem matemática de sistemas quânticos é a de auto adjuntos, cujo espectro é um subconjunto de $\mathbb{R}$, e para os quais sempre existe uma representação espectral.

A técnica utilizada em (HUANG; TARN, 1983) para tratar a questão do domínio dos operadores não limitados é a de definir um subconjunto denso do espaço de estados, para o qual os operadores estão sempre definidos. Com base nos resultados de Nelson, (NELSON, 1959) apud (HUANG; TARN, 1983), os autores utilizam o conjunto dos vetores analíticos do espaço de Hilbert.

Definição 23 (Vetores analíticos de um operador). Um elemento $\omega$ de um espaço de Hilbert $H$ é chamado um vetor analítico de A se a expansão em série de $\exp (s A)$

$$
\sum_{n=0}^{\infty} \frac{\left\|A^{n} \omega\right\|}{n !} s^{n}
$$

tiver um raio de convergência positivo, $s>0$.

O conjunto de vetores analíticos de um operador $A$ é denotado por $\mathcal{D}_{\omega}(A)$

Notar que, se o operador $A$ for limitado, então todos os elementos de $H$ são trivialmente vetores analíticos. Similarmente, pode-se definir o conjunto de vetores analíticos para álgebras de operadores.

$\overline{11}$ No caso de operadores essencialmente de dimensão infinita, mas com um número infinito (contável) de autovalores, a decomposição espectral pode ser expressa por uma série infinita. Contudo, no caso de operadores com espectro contínuo, a teoria da medida é essencial para definir uma "soma" em um conjunto de "índices" não contável. A soma indicada é uma integral de Lebesgue e os índices são os subconjuntos mensuráveis do espectro do operador. 
Definição 24 (Vetores analíticos de uma álgebra de operadores). Um elemento $\omega$ de um espaço de Hilbert $H$ é chamado um vetor analítico de uma álgebra de Lie, $\mathcal{L}$, se a série

$$
\sum_{n=0}^{\infty} \frac{1}{n !} \sum_{i_{1}<\ldots<i_{d}}\left\|H_{i_{1}}^{n_{1}} \ldots H_{i_{d}}^{n_{d}} \omega\right\| s^{n}, \quad n_{1}+\ldots n_{d}=n
$$

tiver um raio de convergência positivo, $s>0$, para alguma base $\left\{H_{1}, \ldots, H_{d}\right\}$ da álgebra de Lie

O conjunto de vetores analíticos de uma álgebra de operadores $\mathcal{L}$ é denotado por $\mathcal{D}_{\omega}(\mathcal{L})$

O teorema a seguir, devido a Nelson, (NELSON, 1959) apud (HUANG; TARN, 1983), garante a existência de um domínio analíticos para um conjunto de operadores.

Teorema 25 (Existência de domínio analítico). Seja $\mathcal{L}$ uma álgebra de Lie de operadores auto adjuntos em um espaço de Hilbert $H$, cuja base de operadores $\left\{H_{1}, \ldots, H_{d}\right\}$ tenha dimensão finita, d, e também um domínio invariante comum, denso em $H$.

Se o operador $T=\sum_{i=1}^{d} H_{i}^{2}$ for essencialmente auto adjunto com extensão em $H$ denotada por $\bar{T}$, então

1. existe um grupo unitário $\Gamma$ em $H$ com álgebra de Lie dada por $\mathcal{L}$,

2. os vetores analíticos de $\bar{T}$ serão vetores análiticos de toda álgebra $\mathcal{L}$

3. o domínio analítico $\mathcal{D}_{\omega}(\mathcal{L})$ é invariante em $\Gamma$ e denso em $H$.

Demonstração. A prova desse teorema pode ser encontrada em (NELSON, 1959) apud (HUANG; TARN, 1983).

Deve-se notar o importante fato de que o teorema de existência de Nelson é válido para uma álgebra de operadores de dimensão finita. Assim, a subvariedade de $M \in H$, invariante pela aplicação dos elementos da álgebra $\mathcal{L}$, possui dimensão finita pelo teorema de Frobenius. A variedade diferenciável $M$ é definida como o fecho do conjunto alcançável $\mathcal{R}_{t}$ na topologia induzida de $H$.

$$
M=\mathcal{R}\left(\psi_{0}\right)=c l_{\left|s_{i}\right| \rightarrow \infty}\left\{e^{s_{0} H_{0}} \ldots e^{s_{r} H_{r}} \psi_{0} \mid s_{i} \in \mathbb{R}\right\}
$$

em que $\psi_{0}$ é um elemento do domínio comum, $\mathcal{D}_{\omega}\left(\left\{H_{0} \ldots H_{r}\right\}_{L . A .}\right)$, dos operadores auto adjuntos que geram a álgebra de Lie indicada. Nota-se nesse ponto que os autores em (HUANG; TARN, 1983) não buscam uma caracterização para o conjunto alcançável. Estritamente, o conjunto alcançável deve ser definido a partir de um ponto do espaço, $\psi_{0}$ no caso, e uma vez que $M \neq H$, ou seja o fluxo do sistema dinâmico não é capaz de 
alcançar ou se aproximar de todos os pontos do espaço de estados, a notação mais correta deve ser $M_{\psi_{0}}$

Pode-se definir também o conjunto alcancável para um tempo finito.

$$
\mathcal{R}_{t}\left(\psi_{0}\right)=\operatorname{cl}\left\{e^{s_{0} H_{0}} \ldots e^{s_{r} H_{r}} \psi_{0} \mid s_{1}+\ldots+s_{r}=t\right\}
$$

Seguem abaixo algumas definições bastante restritivas de controlabilidade.

Definição 26 (Controlabilidade analítica forte). Um sistema dinâmico quântico definido por uma álgebra dinâmica, $\mathcal{L}=\left\{H_{0} \ldots H_{r}\right\}_{L . A}$., possui a propriedade da controlabilidade analítica forte em $M \subset S_{H}$, uma subvariedade da esfera unitária de $H$, se, para todo $t>0$ e para todo $\psi \in \mathcal{D}_{\omega} \cap M, \mathcal{R}_{t}(\psi)=\mathcal{D}_{\omega} \cap M$.

Definição 27 (Controlabilidade analítica fraca). Um sistema dinâmico quântico definido por uma álgebra dinâmica, $\mathcal{L}=\left\{H_{0} \ldots H_{r}\right\}_{L . A}$. possui a propriedade da controlabilidade analítica em $M \subset S_{H}$, uma subvariedade da esfera unitária de $H$, se, para algum $t \leq \infty$ e para todo $\psi \in \mathcal{D}_{\omega} \cap M, \mathcal{R}_{t}(\psi)=\mathcal{D}_{\omega} \cap M$.

A restrição das definições acima está associada a um vínculo desnecessário à definição de controlabilidade existente para sistemas de dimensão finita, caso em que a dinâmica permite a controlabilidade exata. Essa restrição está também vinculada a uma conclusão um pouco precipitada estabelecida pelos autores ao final do artigo (HUANG; TARN, 1983), na qual praticamente eliminam a possibilidade de haver controlabilidade em uma dinâmica essencialmente infinita. Tal conclusão está, em nossa visão, equivocada, pois estaria vinculada a um elemento do espaço de Hilbert $L^{2}(\mathbb{R})$ similar a uma função com suporte não compacto, para a qual a norma induzida pelo produto escalar é infinita. Em $\ell^{2}$, estaria vinculada a um vetor cuja série definida por sua norma seria infinita. Em aproximação de funções por séries de Fourier, é sabido que, satisfeitas determinadas condições, o erro na aproximação pode ser tão pequeno quanto desejado. Assim, a série de Fourier converge para a função aproximada se o número de termos for infinito.

Conforme observação já realizada sobre o teorema 25 (Nelson) sua validade está vinculada a uma álgebra de Lie de dimensão finita, portanto, o conjunto alcançável a partir de um determinado ponto do espaço de estados é uma subvariedade de dimensão finita. Em particular, valem os teoremas de Frobenius e de Chow, a partir dos quais são construídos os demais teoremas de teoria de controle clássica.

Com base nessa argumentação, após trabalharem na relação existente entre campos de vetores em espaços de dimensão finita e operadores auto adjuntos de uma álgebra de Lie dinâmica, os autores em (HUANG; TARN, 1983) transportam para os sistemas dinâmicos quânticos fechados os teoremas da teoria de controle. É importante notar que os sistemas dinâmicos quânticos modelados não são triviais. Uma vez que os autores abordam os 
operadores não limitados, comuns na física quântica, e tratam adequadamente a questão do domínio, esse artigo é um dos pilares da teoria de sistemas dinâmicos quânticos. Seus resultados fundamentais, além daqueles já indicados acima, encontram-se a seguir. O teorema 28 a seguir tem sua formulação baseada em (WU; TARN; LI, 2006).

Teorema 28 (Critério de Controlabilidade Analítica). Seja $\mathcal{U}=\left\{H_{0} \ldots H_{r}\right\}_{L . A .}$ uma álgebra dinâmica de dimensão finita para a qual esteja definido um domínio analítico $\mathcal{D}_{\omega}$. Sejam $\mathcal{B}=\left\{H_{1} \ldots H_{r}\right\}_{\text {L.A. }}$ e $\mathcal{C}=\left\{a d{ }_{H_{0}}^{k} \mathcal{B} \mid k \in \mathbb{N}\right\}_{\text {L.A. }}$, em que ad ${ }_{H_{0}}^{0} \mathcal{B}=\mathcal{B}$ e ad ${ }_{H_{0}}^{k+1} \mathcal{B}=\left[H_{0}, a d \underset{H_{0}}{k}\right]$.

Se

1. $[\mathcal{B}, \mathcal{C}] \subseteq \mathcal{B}$

2. Para todo $\psi \in M \cap \mathcal{D}_{\omega}$ a dimensão de $\mathcal{C}(\psi)$ é igual à dimensão de $M$

Então, o sistema dinâmico quântico possui a propriedade da controlabilidade analítica forte em cada uma das subvariedades $M_{\psi_{0}}$, para algum $\psi_{0} \in M \cap \mathcal{D}_{\omega}$.

Demonstração. A prova desse teorema pode ser encontrada em (HUANG; TARN, 1983).

Teorema 29 (Controlabilidade analítica em $S_{H}$ ). Se $\left\{H_{0} \ldots H_{r}\right\}$ geram uma álgebra de Lie dinâmica, $\mathcal{L}$, de dimensão finita, $d$, que admite um domínio analítico $\mathcal{D}_{\omega}$, então o sistema dinâmico quântico associado não é analiticamente controlável em $S_{H}$.

Demonstração. A prova deste teorema pode ser encontrada em (HUANG; TARN, 1983).

Corolário 30. Se um sistema dinâmico quântico é analiticamente controlável em $S_{H}$, então, sua álgebra de Lie dinâmica, $\mathcal{L}$, deve ter dimensão infinita.

Demonstração. A prova desse corolário pode ser encontrada em (HUANG; TARN, 1983).

A partir do corolário acima, os autores realizam uma análise sobre a forma de uma sequência de comandos de controle em uma álgebra de Lie de dimensão infinita. Tomam, contudo, como paradigma de análise, as definições de controlabilidade forte e fraca, que consideramos restritivas em análise acima. É nosso pensamento que a noção mais adequada para a controlabilidade em espaços de dimensão infinita deve ser feita em termos de convergência, ou seja, por aproximação. Isso é o que foi realizado em (WU; TARN; LI, 2006). Adicionalmente, uma importante motivação para a extensão realizada é que a inexistência de um domínio analítico já foi demonstrada para uma classe considerável 
das álgebras de Lie de dimensão infinita, vide (ARNAL, 1976) apud (WU; TARN; LI, 2006).

Uma consequência interessante do trabalho de Huang, Tarn e Li é que seus resultados permitem uma generalização para o caso de sistemas não lineares de dimensão infinita. ${ }^{12}$

Uma das possíveis generalizações para a teoria desenvolvida em (HUANG; TARN, 1983), apresentada pelos autores em sua conclusão, é a ampliação do domínio analítico, $\mathcal{D}_{\omega}$, para o domínio dos vetores diferenciáveis $\mathcal{D}_{\infty}$, sujeitos a condições de existência menos restritivas. Esse é o cerne do trabalho desenvolvido em (WU; TARN; LI, 2006). Digno de nota é o nível da abstração matemática necessário para desenvolver uma teoria que comporte o tratamento de álgebras e grupos de Lie de dimensão infinita. Em virtude dessa dificuldade, surge naturalmente uma disposição a reavaliar se o caminho trilhado é o mais adequado. E se a generalização buscada não é apenas um mero exercício de matemática aplicada.

No entanto, considerando o nível de desenvolvimento dos experimentos reais em óptica quântica e em circuitos quânticos com junções de Josephson, ambos sistemas que são modelados por sistemas dinâmicos quânticos de dimensão infinita, é necessário o esforço. Deve-se ressaltar, contudo, que a aproximação da dinâmica dos sistemas acima por estados gaussianos apresenta bons resultados para os sistemas quânticos abertos, ou seja, a aplicação prática dos resultados desenvolvidos para sistemas dinâmicos estocásticos tem tido sucesso. Em suma, tendo em vista os dispositivos físicos citados, é importante que se tome nota dos desenvolvimentos abstratos da teoria de controle quântico para álgebras de Lie de dimensão infinita.

Considerando que existem fenômenos quânticos que não possuem paralelo nos domínios da física clássica, a hipótese de a teoria quântica de sistemas dinâmicos possuir uma estrutura adicional não é descartável a priori. E uma vez que a caracterização dos fenômenos relacionados à medição e ao emaranhamento e de seus efeitos na dinâmica e no controle de sistemas não está completa, é fundamental a existência de uma base teórica sólida, sobre a qual os desenvolvimentos próprios da teoria quântica de sistemas dinâmicos possam ser aferidos. Essa base teórica será também uma fonte adicional de inspiração para a busca de soluções para os problemas que a teoria poderá colocar ao longo de seu desenvolvimento e maturação.

Os autores iniciam em (WU; TARN; LI, 2006) com uma caracterização dos sistemas dinâmicos quânticos que são tratáveis pela teoria por eles desenvolvida. Trata-se da

12 Mesmo para os casos em que a equação diferencial deixa de ser linear com relação ao estado do sistema, noção que poderíamos denominar não linearidade forte, em contraposição à noção de não linearidade fraca, quando a dinâmica decorrente da Hamiltoniana do sistema clássico equivalente é do tipo não linear. 
categoria dos sistemas quânticos que possuem uma álgebra Lie de simetria com dimensão finita, $\mathcal{L}=\left\{L_{1} \ldots L_{d}\right\}_{L . A .}$. Assume-se que a álgebra de Lie dinâmica possa ser expressa por polinômios antissimétricos dos operadores que geram a álgebra de Lie de simetria. Esses elementos definem uma estrutura algébrica denominada álgebra universal abrangente, tradução livre do termo em inglês universal enveloping algebras, indicada por $E(\mathcal{L})$.

Um teorema citado pelos autores, devido a Poincaré-Birkhoff-Witt, informa que uma base para uma álgebra universal abrangente, $E(\mathcal{L})$, é dada pelo conjunto

$$
\left\{L_{1}^{\alpha_{1}} \ldots L_{d}^{\alpha_{d}} \mid \alpha_{i} \in \mathbb{N}\right\}
$$

Denote-se por $E^{(n)}(\mathcal{L})$ o subespaço de $E(\mathcal{L})$ dado pelos elementos que podem ser expressos por elementos da base cuja ordem não seja superior a $n$. Obtém-se assim uma estrutura de álgebra gradual ou graduada, tradução livre do termo graded álgebra, indicada por

$$
E^{(1)}(\mathcal{L}) \subset E^{(2)}(\mathcal{L}) \subset \ldots \subset E^{(n)}(\mathcal{L}) \subset \ldots,
$$

que decompõe o espaço vetorial de dimensão infinita $E(\mathcal{L})$ em subespaços de dimensão finita encaixados entre si.

Nesse ponto, os autores afirmam, acreditamos que com razão, que a estrutura algébrica usada com frequência em física quântica facilita os cálculos, uma vez que substitui operadores diferenciais por operações algébricas. Esta é a prática de escrever o Hamiltoniano de oscilador harmônico quântico usando a notação

$$
H_{0}=\frac{1}{2}\left(X^{2}+P^{2}\right)
$$

em vez de escrever

$$
H_{0}=\frac{1}{2}\left(x^{2}-\frac{\partial^{2}}{\partial x^{2}}\right)
$$

Em três exemplos apresentados, todos eles baseados em simetrias angulares do potencial de Pöschl-Teller e na álgebra $s u(1,1)$, os autores demonstram como a álgebra de simetria pode ter uma influência decisiva nas dimensões da álgebra dinâmica. Importante notar nos exemplos discutidos que os autores utilizam o conceito de conjuntos de observáveis, que é um método de análise comum na mecânica quântica, a partir do qual observáveis compatíveis são diagonalizados em conjunto. Há, portanto, uma conexão entre os resultados de (PRUGOVECKI, 1981) analisados anteriormente, por exemplo, o teorema 17, e as análises realizadas em (WU; TARN; LI, 2006).

Em um primeiro exemplo, os autores apresentam um sistema com álgebra dinâmica finita e destacam como o caso apresentado se diferencia do caso em que o espaço de estados possui dimensão finita. Isso porque no caso tratado, o subespaço vetorial é formado por operadores não limitados e as soluções da equação de Schrödinger, uma equação diferencial parcial, são funções. 
No caso em que os autores tratam os estados espalhados, scattering states, do sistema, o espectro obtido é contínuo e o resultado obtido é que a álgebra de Lie dinâmica vinculada a um sistema que possui "autoestados" indexados por um índice contínuo, possui dimensão infinita. O operador que possui espectro contínuo é o operador da álgebra de simetria que foi definido como não compacto.

O terceiro exemplo, os autores mostram que uma álgebra de operadores de dimensão infinita também pode ser gerada por uma álgebra de simetria formada a partir de operadores compactos. Nesse caso, porém, o espectro dos operadores diagonalizados é discreto.

Os exemplos motivam fisicamente, portanto, a definição do domínio de trabalho realizada a seguir, pela qual os monômios do teorema de Poincaré-Birkhoff-Witt, que constituem os elementos da base do espaço vetorial $E(\mathcal{L})$, admitem os vetores selecionados como parte de seu domínio.

Definição 31 (Domínio diferenciável). Um elemento $\phi$ de um espaço de Hilbert $H$ é chamado um vetor diferenciável de um conjunto gerador, $\left\{L_{1}, \ldots, L_{d}\right\}$, se pertence ao conjunto

$$
\mathcal{D}_{\infty}=\left\{\phi \in H \mid\left\|L_{1}^{s_{1}}, \ldots, L_{d}^{s_{d}} \phi\right\|<\infty \quad ; \forall s_{i} \in \mathbb{N}\right\}
$$

O conjunto descrito acima é chamado domínio diferenciável, em inglês, smooth domain.

O domínio diferenciável definido para um conjunto de geradores de uma álgebra de simetria é bem definido e invariante para todos os operadores de uma álgebra abrangente universal $E(\mathcal{L})$. Lembrando que os operadores da álgebra dinâmica são tomados da álgebra $E(\mathcal{L})$, o problema de controle quântico em dimensão infinita está bem colocado para a classe de problemas analisada.

Uma forma alternativa de definir o domínio diferenciável é por meio da definição de um espaço de limite inverso de Hilbert, espaço ILH ou inverse limit Hilbert space. Tratase de uma engenhosa estrutura de espaços encaixados que tem por limite o conjunto de vetores diferenciáveis $\mathcal{D}_{\infty}$. É por meio dessa estrutura, altamente complexa e abstrata, que será definido um grupo de transformação do espaço $\mathcal{D}_{\infty}$, que é obtido por meio da exponenciação dos elementos da álgebra abrangente universal.

Outra estrutura matemática está relacionada aos vetores diferenciáveis. São os espaços de Hilbert equipados Rigged Hilbert Spaces ou Gelfand Triple, que utilizam a definição e as propriedades dos espaços duais para caracterizar uma cadeia de espaços tal que

$$
\mathcal{D}_{\infty} \subset H \subset \mathcal{D}_{\infty}^{*}
$$

e $\mathcal{D}_{\infty}$ é denso em $H$ que é denso em $\mathcal{D}_{\infty}^{*}$. 
Pode-se encontrar artigos e até monografias sobre física quântica que defendem os espaços de Hilbert equipados como a estrutura matemática ideal para tratar os aspectos da teoria relacionados aos operadores não limitados, vide (MADRID, 2005; BOHM, 1994).

Definição 32 (Conjunto Alcançável). Um estado quântico $\psi_{f}$ é alcançável a partir de outro estado quântico $\psi_{0}$ se existe um instante de tempo $T$ e um conjunto de controles admissíveis definido no intervalo $[0, T], u_{i}:[0, T] \mapsto \mathbb{R}$, que leva o sistema dinâmico quântico do estado $\psi(0)=\psi_{0}$ ao estado $\psi(T)=\psi_{f}$. O conjunto dos estados alcançáveis a partir de $\psi_{0}$ para qualquer instante de tempo é denotado por $\mathcal{R}\left(\psi_{0}\right)$ e o conjunto dos estados alcançáveis a partir de $\psi_{0}$ até um determinado instante de tempo $t$ é denotado por $\mathcal{R}_{t}\left(\psi_{0}\right)$. O conjunto dos estados alcançáveis em tempo infinitesimal a partir de $\psi_{0}$ é denotado por $\mathcal{R}_{0}\left(\psi_{0}\right)=\bigcap_{t>0} \mathcal{R}_{t}\left(\psi_{0}\right)$.

Definição 33 (Variedade M). Seja $M_{\psi_{0}}$ o fecho topológico do seguinte conjunto

$$
\left\{e^{s_{k} H_{\alpha_{k}}} \ldots e^{s_{1} H_{\alpha_{1}}} \psi_{0} \mid s_{k} \in \mathbb{R}, \alpha_{k} \in\{1 \ldots m\}, k \in \mathbb{N}\right\}
$$

definido para um sistema dinâmico quântico com um Hamiltoniano livre, $H_{0}$, e $m$ Hamiltonianos de controle, $H_{i}$.

O conjunto dos estados alcançáveis a partir de $\psi_{0}, \mathcal{R}\left(\psi_{0}\right)$, está contido na variedade $M_{\psi_{0}}$, pois nela, por definição, estão representados todos os controles admissíveis. ${ }^{13}$ Tendo em vista que a álgebra de controle $\mathcal{U}=\left\{H_{0} \ldots H_{m}\right\}_{L A}$ pode apresentar dimensão infinita, é possível que a variedade $M$ definida tenha também dimensão infinita. ${ }^{14}$

As definições de controlabilidade exata desenvolvidas em (HUANG; TARN, 1983) para o domínio analítico podem ser adaptadas para o domínio diferenciável. São desenvolvidas novas definições de controlabilidade aproximada, que apresentamos a seguir.

Definição 34 (Controlabilidade diferenciável aproximada). Um sistema de controle quântico apresenta a propriedade da controlabilidade diferenciável aproximada em um conjunto $M_{\psi}$, quando o conjunto alcançável $\mathcal{R}(\psi)$ é denso em $M_{\psi} \cap \mathcal{D}_{\infty}$, com respeito à topologia do espaço ILH, para todo $\psi_{0} \in M_{\psi} \cap \mathcal{D}_{\infty}$.

Definição 35 (Controlabilidade diferenciável aproximada forte). Um sistema de controle quântico apresenta a propriedade da controlabilidade diferenciável aproximada forte em

$\overline{13}$ Na teoria desenvolvida em (WU; TARN; LI, 2006), os controles admissíveis são funções constantes por partes, com um conjunto enumerável de descontinuidades.

14 No artigo (WU; TARN; LI, 2006) não é realizada uma caracterização topológica da variedade $M$ com relação ao espaço de Hilbert, $H$, dos estados do sistema quântico. Assim, mesmo que $M$ tenha dimensão infinita, não é sabido se é um conjunto denso no espaço no espaço de Hilbert. Como consequência, as noções de controlabilidade apresentadas são limitadas. Não se trata de uma controlabilidade no espaço de estados inteiro, mas sim de uma controlabilidade em conjuntos possivelmente limitados $M_{\psi_{0}}$, para $\psi_{0} \in H$. É por esse motivo que introduzimos na notação o subescrito, que se encontra apenas subentendido no artigo original. 
um conjunto $M_{\psi}$, quando o conjunto alcançável $\mathcal{R}_{t}(\psi)$ é denso em $M_{\psi} \cap \mathcal{D}_{\infty}$, com respeito à topologia do espaço ILH, para todo $\psi_{0} \in M_{\psi} \cap \mathcal{D}_{\infty}$ e para todo $t>0$.

O problema de controle é abordado diretamente no grupo de Lie dos propagadores unitários, por meio do estudo da equação

$$
\dot{U}(t)=\left[H_{0}+\sum_{j=1}^{m} u_{j}(t) H_{j}\right] U(t) \quad, \quad U(0)=I .
$$

O propagador do sistema $U(t)$ é dependente das funções de controle $u_{j}(t)$ e pertence ao grupo de Lie, $\mathcal{G}$, gerado pela álgebra de controle $\mathcal{U}$ que pode ter dimensão infinita. Nessa notação, $M_{\psi_{0}}$ é a órbita de $\mathcal{G}$ passando por $\psi_{0}$. Controlabilidade nesse contexto é equivalente à possibilidade de gerar um conjunto de propagadores denso em $\mathcal{G}$. Isso não é equivalente à possibilidade de gerar uma matriz unitária qualquer, pois em princípio nada se pode aferir a respeito das propriedades topológicas de $\mathcal{G}$ com relação ao conjunto de todas as matrizes unitárias de $H$. Na realidade, os sistemas dinâmicos quânticos analisados em (HUANG; TARN, 1983; WU; TARN; LI, 2006) definem uma foleação no espaço de estados. Em (HUANG; TARN, 1983), por exemplo, que trata de variedades integrais de dimensão finita, $d$, o espaço de estados é dividido em folhas $d$-dimensionais. Em ambos os casos, a controlabilidade analisada é restrita a essas folhas. A equivalência entre os conceitos de controlabilidade, relacionado ao sistema dinâmico quântico, e universalidade, relacionado à informação e computação quântica, existe quando há a possibilidade de gerar, mesmo que de forma aproximada, um operador unitário qualquer no espaço de Hilbert, associado tanto ao sistema dinâmico quanto ao sistema de informação quântica.

Na teoria desenvolvida em (WU; TARN; LI, 2006), os autores fundamentam seus teoremas no teorema de Frobenius e nas fórmulas de Campbell-Baker-Hausdorff e de Trotter. De acordo com a apresentação feita no artigo, e com as referências citadas, todos esses resultados fundamentais foram extendidos para dimensão infinita. Apesar do nível de abstração utilizado para realizar as definições de domínio para a colocação do problema, os argumentos relacionados aos resultados principais estão associados a uma linguagem matemática que não é estranha à teoria de controle geométrico.

Parte-se da definição de operadores Hamiltonianos fortemente emuláveis, que representam operadores para os quais os respectivos propagadores unitários podem ser gerados em intervalos de tempo arbitrariamente pequenos.

Definição 36 (Hamiltoniano fortemente emulável). Um operador Hamiltoniano $X$ é fortemente emulável se sua curva integral $\left\{e^{(t X)} \psi \mid t \in \mathbb{R}\right\}$ está contida no fecho topológico de $\mathcal{R}_{0}(\psi)$.

Um conjunto de Hamiltonianos é fortemente emulável se cada um de seus elementos é fortemente emulável. 
A seguir encontram-se os principais resultados demonstrados em (WU; TARN; LI, 2006).

Lema 37 (Hamiltonianos fortemente emuláveis). Sejam $X$ e $X_{1} \ldots X_{\alpha}$ Hamiltonianos fortemente emuláveis. Então

1. $\left(X_{i_{n}}\right)_{s_{n}} \ldots\left(X_{i_{1}}\right)_{s_{1}} \psi \in \overline{\mathcal{R}}_{0}(\psi)$, para $n \in \mathbb{N}, i_{1} \ldots i_{n} \in\{1 \ldots \alpha\}, s_{i} \in \mathcal{R}$ e $\psi \in \mathcal{D}_{\infty}$.

2. $\left(H_{0}\right)_{t} X_{s} \psi \in \overline{\mathcal{R}}_{t}(\phi)$ e $X_{s}\left(H_{0}\right)_{t} \psi \in \overline{\mathcal{R}}_{t}(\phi)$, para todo $\psi \in \mathcal{R}_{t}(\phi), s \in \mathcal{R}$ e $t>0 .{ }^{15}$

Lema 38 (Hamiltonianos fortemente emuláveis). O Hamiltoniano $X$ é fortemente emuláveis se, e somente se, $\left(H_{0}+c X\right)_{t} \psi \in \overline{\mathcal{R}}_{t}(\psi)$, para todo $t \in \mathbb{R}$.

Lema 39 (Hamiltonianos fortemente emuláveis). O conjunto $\mathcal{U}_{S}$ dos Hamiltonianos fortemente emuláveis é uma álgebra de Lie contendo $\mathcal{B}=\left\{H_{1} \ldots H_{r}\right\}_{\text {L.A. }}$.

Teorema 40 (Caracterização da álgebra $\mathcal{C}$ ). A álgebra de Lie

$$
\mathcal{C}=\left\{a d_{H_{0}}^{k} \mathcal{B} \mid k \in \mathbb{N}\right\}_{L . A}
$$

é fortemente emulável se $[\mathcal{B}, \mathcal{C}] \subseteq \mathcal{B}$.

Teorema 41 (Caracterização da variedade integral de $\mathcal{C})$. Seja $I(\psi, \mathcal{C})$ a variedade integral maximal de $\mathcal{C}$ contendo $\psi$ e $I_{t}(\psi, \mathcal{C})=\left(H_{0}\right)_{t} I(\psi, \mathcal{C})=I(\psi, \mathcal{C})\left(H_{0}\right)_{t}$, então ${ }^{16}$

$$
\mathcal{R}_{t}(\psi)=I_{t}(\psi, \mathcal{C})
$$

Teorema 42 (Critério de controlabilidade em domínio diferenciável). O sistema dinâmico quântico 3.37 possui a propriedade da controlabilidade aproximada forte se as seguintes condições são satisfeitas:

1. $[\mathcal{B}, \mathcal{C}] \subseteq \mathcal{B}$

2. $\mathcal{C}(\phi)=\mathcal{U}(\phi)$ para todo $\phi \in M \cap \mathcal{D}_{\infty}$ e as álgebras $\mathcal{C}$ e $\mathcal{U}$ possuem dimensão infinita.

Os resultados de (WU; TARN; LI, 2006) apresentados acima são bastante fortes. Apesar de terem a limitação de se restringirem a variedades integrais em princípio desconexas entre si, ou seja, a foleações, os resultados tratam dos problemas dinâmicos em dimensão infinita com a possível presença de operadores não limitados.

\footnotetext{
15 Para facilitar a notação ocorre uma identificação entre os Hamiltonianos e os propagadores por eles gerados.

16 O enunciado deste teorema foi ligeiramente ampliado a partir de sua versão original no artigo. inicialmente os autores propuseram a tese $\mathcal{R}_{t}(\psi) \subseteq I_{t}(\psi, \mathcal{C})$. No entanto, como a segunda parte da igualdade é provada no teorema seguinte, enunciamos o teorema de forma completa.
} 
No entanto, analisando inicialmente a teoria e as demonstrações dadas para os lemas e teoremas, parece que os autores criaram, de forma artificial, o conceito de Hamiltoniano fortemente emulável. Esta dúvida surge quando é apresentada a segunda parte do lema 37, pois parece que o conjunto de Hamiltonianos fortemente emuláveis não é capaz de reproduzir a dinâmica livre do sistema. ${ }^{17}$ Assim, a controlabilidade do sistema estaria reduzida à possibilidade de modificar o estado do sistema em um intervalo de tempo infinitesimal. Nesse caso, a teoria apresentada no artigo estaria associada a um sistema físico de energia não limitada. Do ponto de vista da engenharia este é um resultado que possui pouca utilidade. É importante, no entanto, do ponto de vista matemático, pois mostra que o problema possui solução. Aponta também diversos caminhos para buscar um resultado mais forte, por exemplo, estudar as condições sob as quais o Hamiltoniano livre possui a propriedade de ser também fortemente emulável.

Esse caso seria equivalente na teoria de controle clássico ao cancelamento de zeros e pólos. Assim, havendo a possibilidade de forçar assintoticamente a dinâmica livre do sistema de forma a cancelar suas frequencias naturais e impor outras, estaríamos mais próximos de uma teoria de controle de sistemas dinâmicos quânticos que pode ser usada para calcular trajetórias ótimas, que ao final, o principal objetivo do controle de sistemas.

Uma questão que chama bastante a atenção em (WU; TARN; LI, 2006) é que, após a apresentação dos resultados, os exemplos discutidos envolvem não linearidades de forma essencial, ou seja, essas não linearidades é que são responsáveis por gerar uma álgebra dinâmica de dimensão infinita, condição essencial para que o sistema quântico seja controlável em sua variedade integral. ${ }^{18}$ Como a controlabilidade está relacionada à transitividade do grupo de transformações, gerado pela dinâmica do sistema, sobre um determinado conjunto de interesse, é inevitável que seja feita uma relação entre a não linearidade de um sistema quântico e sua transitividade em um conjunto de interesse.

Essa questão é estudada em sistemas dinâmicos como uma das condições formais para existência de caos determinístico, a transitividade topológica, caracterizada pela existência de uma solução da equação diferencial cuja trajetória é densa no espaço de estados.

No caso da dinâmica de sistemas quânticos - pelos exemplos apresentados pelo autores (WU; TARN; LI, 2006) para ilustrar o critério de controlabilidade por eles desenvolvido - sem não linearidade não existe álgebra dinâmica de dimensão infinita, portanto, não há condições para que o sistema seja controlável, ou seja, não há transitividade em um determinado conjunto de interesse. ${ }^{19}$

$17 \quad$ Essa análise deve ser realizada de forma mais minuciosa, o que é bastante difícil, tendo em vista o nível de abstração necessário para definir o problema, considerando a utilização dos vetores diferenciáveis e de inverse limit Hilbert spaces, que conduzem as análises a um espaço vetorial topológico.

18 Trata-se de não linearidades em sentido fraco.

19 A questão da não linearidade em sistemas quânticos é intrigante, pois a equação de Schrödinger é 
O primeiro dos exemplos apresentados é inspirado na arquitetura desenvolvida por Lloyd and Braunstein (LLOYD; BRAUNSTEIN, 1999) apud (WU; TARN; LI, 2006) para realizar processamento quântico a partir de variáveis quânticas contínuas. Essa arquitetura estaria baseada na utilização de quatro Hamiltonianos de controle que atuam em um sistema equivalente a um oscilador harmônico quântico. De acordo com a proposta de processamento quântico apresentada por Lloyd and Braunstein, a partir do controle dos quatro Hamiltonianos, qualquer polinômio nos operadores posição e momento pode ser emulado dinamicamente. A partir dessa propriedade, os autores conseguem demonstrar que sua arquitetura de computação é universal, ou seja, que o sistema dinâmico em que o computador quântico é baseado é controlável. O Hamiltoniano não linear é essencial porque é a partir dele que a álgebra dinâmica é enriquecida com infinitos termos independentes entre si.

O segundo exemplo é baseado em sistemas de partículas em um potencial de Pöschl-Teller e é relacionado a momentos angulares. Inicialmente, a dinâmica apresentada possui uma álgebra dinâmica de dimensão finita, mas após a adição de um termo quadrático no conjunto de Hamiltonianos de controle, a álgebra dinâmica passa a ter dimensão infinita. Como se trata de um termo quadrático no Hamiltoniano, de início se poderia pensar que é um termo linear. No entanto, tomando por base a analogia de Schwinger entre sistemas angulares e osciladores quânticos, vide por exemplo (SAKURAI, 1994, pp. 217 e ss.), vemos que os operadores de momento angular são equivalentes a termos quadráticos dos operadores de posição e de momento. Portanto, um operador de momento angular quadrático já é um termo não linear, na nomenclatura em uso na física e já mencionada.

Dessa forma, a relação entre Hamiltonianos não lineares, em sentido físico, e transitividade dinâmica permanece para ser investigada. Nesse sentido, um importante resultado da óptica quântica parece interessante: em 2001, Knill, Laflamme e Milburn (KNILL; LAFLAMME; MILBURN, 2001) demonstraram que circuitos ópticos baseados apenas em elementos lineares constituem uma arquitetura de hardware universal para computação quântica. Em função da equivalência entre os conceitos de universalidade, relacionado à arquitetura computacional, ou seja, ao processamento quântico da informação, e de controlabilidade, relacionado à dinâmica do sistema físico, poderia se tratar de um contra exemplo. No entanto, a arquitetura baseada em óptica linear utiliza sistemas de dimensão finita, estados de polarização de fótons. A situação discutida acima é presente em

\footnotetext{
linear, pois é baseada em operadores lineares, e o princípio da superposição de estados é válido. No entanto, o termo linear é aplicado no contexto da física quântica para sinalizar Hamiltonianos que possuem equivalente na física clássica e que possuem termos quadráticos ou lineares dos operadores posição e momento linear, vide por exemplo (WISEMAN; MILBURN, 2010, pp. 315 e ss.). Nesse caso, na perspectiva dinâmica de Heisenberg um vetor de estado $\hat{\mathbf{x}}$, que é função das posições e momentos característicos de um sistema subjacente, obedece uma equação de movimento da forma $d \hat{\mathbf{x}}=A \hat{\mathbf{x}} d t$.
} 
sistemas de controle quânticos de dimensão infinita.

Por outro lado, sendo esses os principais resultados sobre a controlabilidade geral de sistemas dinâmicos quânticos de que temos conhecimento até este momento, podemos dizer que a teoria ainda é carente de ampliações, generalizações e de resultados novos para que possa suprir a necessidade da engenharia de estados quânticos de sistemas de dimensão infinita. Os artigos de Vilela Mendes (KARWOWSKI; VILELA-MENDES, 2004; VILELA-MENDES, 2009; VILELA-MENDES; MANIKO, 2011) iluminam a estrutura de uma possível solução geral para o problema da controlabilidade, os propagadores dinâmicos essencialmente infinitos, ou seja, operadores unitários capazes de atuar finitamente em todos os elementos da base de um espaço de Hilbert. O exemplo estudado é o operador de shift de coordenadas.

Assim como Vilela-Mendes não estabelece critérios a respeito das álgebras de Lie, $\mathcal{U}, \mathcal{B}$ e $\mathcal{C}$, usualmente estudadas na teoria de controle, também Wu, Tarn e Li não caracterizam o grupo de Lie das transformações unitárias geradas pelas álgebras estudadas na teoria de controle.

A conexão essencial entre as álgebras de controle e um grupo de transformações unitárias denso ou transitivo no espaço de estados ainda falta. Uma parte dessa conexão, no entanto, é dada pelo teorema de Stone, pelo qual, a todo grupo unitário a um parâmetro fortemente contínuo $U(t)$ agindo em um espaço de Hilbert, corresponde um operador auto adjunto $A$, possivelmente não limitado, de tal forma que $U(t)=e^{i t A}$.

Por si só, o teorema de Stone garante que o problema de controle em dimensão infinita possui solução. Dado um conjunto $U_{i}(t)$ de transformações unitárias do espaço de Hilbert, cuja composição seja densa no grupo unitário de dimensão infinita, o conjunto associado de operadores auto adjuntos é controlável.

Os principais avanços de que a teoria necessita neste momento são a caracterização da variedade integral de uma álgebra de controle e o estabelecimento de condições para que a variedade integral seja densa no espaço de estados ou, alternativamente, a caracterização das álgebras de controle que são capazes de gerar grupos de transformações unitárias cuja composição seja densa no grupo unitário do espaço de Hilbert.

\subsection{Conclusão}

Encontrou-se uma analogia entre a controlabilidade de sistemas lineares em dimensão finita e sistemas quânticos bilineares em dimensão infinita.

É possível destacar também que, assim como a teoria de controle geométrico em dimensão finita enfrenta sérios problemas para caracterizar topologicamente seus conjuntos alcançáveis, vide discussão em (JURDJEVIC, 1997), também a teoria de controle de 
sistemas dinâmicos quânticos tem dificuldade em definir um domínio de trabalho denso no espaço a partir do qual seja possível construir um conjunto alcancável sobre a variedade integral da equação diferencial, vide (WU; TARN; LI, 2006).

A análise abaixo sobre medidas espectrais é uma tentativa de buscar uma analogia entre sistemas em dimensão finita e em dimensão infinita, extrapolando a analogia encontrada em (REED; SIMON, 1980). Trata-se de conjecturas livres, para as quais ainda não foi feita uma análise prévia de viabilidade. Em virtude disso, é possível que apontem um caminho promissor, tanto quanto é possível que sejam inúteis.

Uma família espectral define uma medida. Todo operador que comuta com essa família espectral pode ser expresso como uma multiplicação por uma função em um espaço $L^{2}(\mathbb{R}, d \mu)$ construído a partir dessa medida. Também característica de um dado operador auto adjunto é a função que aparece como fator multiplicativo. Para uma dada família de operadores que comutam, deve haver uma mudança de coordenadas fraca que leve uma dada $F(\lambda)$ no integrando à função canônica $F(\lambda)=\lambda$. Uma mudança de coordenadas forte implicaria em uma alteração mais profunda da medida da integral, levando a operadores fora da família inicial de operadores que comutam. ${ }^{20}$

Interessantíssimo na exposição acima é também a caracterização de operadores unitários na equação 3.17 e subsequentes. Verifica-se que a analogia feita com espaços de dimensão finita é compatível, uma vez que uma transformação unitária é uma mudança de coordenadas entre duas bases do espaço, no caso entre duas famílias espectrais. A representação espectral de operadores unitários é um pouco diferente, uma vez que seus autovalores são números complexos de norma unitária. No entanto, um programa de solução para o problema da controlabilidade em dimensão infinita que se baseie na construção da solução, pode ter seu ponto de partida na caracterização de operadores unitários apresentada. Assim, uma álgebra dinâmica é controlável se é capaz de gerar, de forma exata ou aproximada, todas as transformações unitárias do espaço (tanto as diferentes medidas quanto as funções $F(\lambda)$ da equação 3.17, se as medidas não forem suficientes).

Para iniciar uma investigação das medidas e de suas relações com famílias espectrais, é possível considerar a existência de uma família de medidas em espaços compactos, cujas integrais resultantes podem ser usadas para modelar as séries de Fourier. As medidas dessa família, por exemplo $\mu_{n}$, seriam do tipo medidas de Dirac, caracterizadas por amostrar as funções do integrando no percentil 50, $\mu_{0}$, depois em 25 e $75, \mu_{1}$, e assim sucessivamente até obter um conjunto de pontos denso no domínio, $\mu_{n}, n \rightarrow \infty$. Os teoremas de convergência e as propriedades topológicas podem dar as condições de funcionamento da técnica acima. Depois se pode partir para a transformada de Fourier, que

20 Assim, uma "mudança de coordenadas fraca" seria uma transformação unitária capaz de relacionar por similaridade operadores auto adjuntos que comutam, já uma "mudança de coordenadas forte" seria uma transformação unitária que não preserva por similaridade a comutatividade dos operadores auto adjuntos de um determinado conjunto. 
é um operador unitário de dimensão infinita no espaço de Hilbert $L^{2}(\mathbb{R})$. Assim como o teorema de Radon-Nikodym, discutido acima, é aplicável nessa linha de investigação, também a decomposição de Lebesgue pode ser uma ferramenta interessante, pois estabelece uma relação entre medidas equivalente à ortogonalidade. O teorema de Freudenthal é outra ferramente matemática que poderia ser explorada, pois é um resultado abstrato do qual decorrem o teorema de Radon-Nikodym e o teorema espectral. 


\section{Controle de Sistemas Quânticos em Malha Fechada}

Um sistema quântico aberto, da mesma forma que um sistema clássico aberto, é definido pela possibilidade de trocar energia com o meio externo. Todos os sistemas físicos reais são, em maior ou menor grau, sistemas abertos. Alguns sistemas, por apresentarem trocas de energia em intensidade muito inferior ao valor médio da energia trocada dinamicamente nos processos evolutivos de interesse, podem ser modelados por meio de sistemas fechados. Em alguns casos, a discrepância entre os valores previstos pela teoria e os valores reais acabam sendo desprezíveis para algumas aplicações práticas.

Quanticamente, a introdução de modelos abertos torna-se mais fundamental em virtude da impossibilidade de se realizar processos de medição sem causar efeitos dinâmicos consideráveis no sistema. Classicamente também ocorre o efeito de interferência do processo de medição na dinâmica do sistema medido, no entanto, classicamente é quase sempre possível empregar aparatos de medição que interferem de forma desprezível. Nos demais casos, é possível modela a dinâmica do processo de medição e incorporá-la à dinâmica da planta. De acordo com esse modelo, tudo se passará como se a informação a respeito da dinâmica do sistema planta + aparato de medição fosse extraída sem troca de energia. Na prática, a premissa de que seria possível extrair informação sem trocar energia (pelo menos em primeira aproximação) é assumida como verdadeira no estudo de sistemas clássicos. ${ }^{1}$

No sistemas quânticos, a possibilidade de se obter informação está associada necessariamente a uma irreversibilidade no espaço de fases. No limite, em um processo de medição de von Neumann, ao se obter informação a respeito de uma variável dinâmica, perturba-se irreversivelmente o estado desse sistema. A perturbação possui natureza ale-

1 Os princípios físicos da natureza delimitam a forma como a informação pode ser manipulada. Pelo princípio de Landauer, por exemplo, o processo de apagar uma informação é necessariamente dissipativo por envolver uma compressão do espaço de fases. Bennet, por outro lado, propôs o modelo computação reversível como forma de evitar o custo energético de se apagar informação. O exemplo mais marcante que demonstra a natureza física da informação é a solução proposta por Bennet para resolver o paradoxo de Maxwell. Maxwell imaginou um gás em uma caixa com dois compartimentos. A parede que divide os compartimentos A e B possui um orifício que pode ser operado por um ser inteligente. Esse ser, observa as moléculas que se aproximam do orificio e deixa passar as moléculas mais rápidas de A para $\mathrm{B}$ e as mais lentas de B para A. Dessa forma, o compartimento A se tornará mais frio que B, em aparente contradição com a segunda lei da termodinâmica. Bennet observou que o espírito precisará armazenar informações sobre as moléculas. Eventualmente, quando for apagar essa informação gerada para retornar às condições iniciais, esse processo dissipará energia. No balanço termodinâmico teremos necessariamente que considerar a entropia associada à informação gravada ou o custo energético de apagá-la. Preserva-se a consistência da termodinâmica e, ao mesmo tempo, constata-se a natureza física da informação. Vide (PRESKILL, 1998) capítulo 1, (JACOBS, 2014) seção 4.1 . 
atória e a dinâmica induzida no processo se mostra estocástica. A análise qualitativa do princípio da incerteza nos leva ao mesmo resultado. Imaginando-se um par de observáveis não compatíveis e um estado de mínima incerteza a respeito desses dois observáveis, conclui-se que qualquer obtenção de informação a respeito de um dos observáveis - diminuindo a incerteza do observador a seu respeito - irá necessariamente provocar o aumento da incerteza a respeito do outro observável. Esse efeito deve ser fundamentalmente aleatório, pois se fosse determinístico levaria o sistema físico a entrar em contradição com o princípio da incerteza. Esse é o mesmo princípio utilizado para apresentar o princípio da incerteza por meio do microscópio de Heisenberg, vide (CLERK, 2014). Se $\Delta x_{i m p}$ é a imprecisão na medição do observável $\hat{x}$ e $\Delta p_{B A}$ é a perturbação no momento linear da partícula, inserido pela inevitável troca de energia entre o aparato medidor e o sistema medido, então, ambos estão restritos a um limite fundamental

$$
\Delta x_{i m p} \Delta p_{B A} \gtrsim \hbar
$$

Um sistema quântico aberto possui um acoplamento dinâmico entre seu estado interno e o meio ambiente com que troca energia. Esse ambiente externo é chamado reservatório ${ }^{2}$ quando modelado como um sistema térmico complexo com um número muito grande de graus de liberdade e idealmente com modos de energia contínuos. A partir da interação com o ambiente, o sistema sofrerá um processo de dissipação, pelo qual haverá não somente perda de energia, mas também perda de correlação entre estados quânticos superpostos. Os dois processos descritos são independentes entre si e este último é conhecido como decoerência. Esses processos dissipativos são mediados por estados do sistema total formado pela composição do sistema aberto com o meio ambiente. ${ }^{3} \mathrm{Em}$ geral, assume-se que a dinâmica do sistema total é conservativa e, portanto, pode ser modelada por meio de um hamiltoniano adequado. Como consequência dos axiomas quânticos, a composição de sistemas ocorre por meio do "produto" ${ }^{4}$ e não por meio da

2 Os termos reservatório, banho ou banho térmico são utilizados por alguns autores para ambientes externos ao sistema de interesse em estado de equilíbrio térmico (BREUER; PETRUCCIONE, 2002). Outros autores preferem utilizar os termos reservatório, banho e banho térmico indiscriminadamente (WISEMAN; MILBURN, 2010).

3 Os processos dissipativos estão intimamente relacionados a processos de flutuação térmica em sistemas físicos. Pelo teorema da flutuação-dissipação (CALLEN; WELTON, 1951), a cada processo de dissipação corresponde um processo de flutuação. Por exemplo, no movimento browniano, a força flutuante e a força de arrasto fluidodinâmico estão relacionados à mesma constante de dissipação relacionada à viscosidade. Em circuitos elétricos, o ruido de Nyquist-Johnson está relacionado à resistência elétrica. Interessante notar que os processos de flutuação térmica também possuem natureza estocástica.

4 A composição de sistemas por produto tensorial dos sistemas cria uma forte vinculação entre os graus de liberdade originais dos sistemas componentes, além de aumentar em geral as dimensões do sistema produto. Essa vinculação, como já apresentado, manifesta-se por meio dos fenômenos de emaranhamento. Pode-se dizer, então, que o acoplamento de um sistema aberto com seu ambiente acarretará, mediante a troca de energia, a criação de emaranhamento entre os graus de liberdade do sistema e os graus de liberdade do ambiente. 
"soma" dos sistemas, como ocorre classicamente.

$$
\rho_{\text {Total }}(0)=\rho_{\text {Sistema }}(0) \otimes \rho_{\text {Ambiente }}(0)
$$

E a dinâmica é induzida pelo hamiltoniano composto.

$$
H_{\text {Total }}(t)=H_{\text {Sistema }} \otimes I_{\text {Ambiente }}+I_{\text {Sistema }} \otimes H_{\text {Ambiente }}+H_{\text {interação }}(t)
$$

O ambiente indicado nesse modelo bipartido pode ser um sistema complexo em um estado térmico do qual se conhecem apenas características macroscópicas ou também pode ser o modelo de um aparato físico que troca energia com o sistema de forma definida e/ou controlada. Nesse último caso, o ambiente poderá ser um modelo para um aparato de medição. De fato, existe um paralelo muito bem definido na teoria quântica entre medição e processos dissipativos. As mesmas equações que modelam a medição, podem ser utilizadas para modelar os processos dissipativos, (JACOBS, 2014) seção 3.6.

Em geral, a dinâmica de um sistema aberto não pode ser modelada por meio de uma evolução temporal unitária. Tal fato decorre da não conservação da energia. A dinâmica pode, então, ser formulada em termos da evolução do operador densidade. As equações decorrentes são chamadas de master equations. A evolução pode ser ou não do tipo markoviana.

Uma discussão a respeito da terminologia é importante neste momento, pois há diferentes definições para master equation. Uma nota no livro de (KAMPEN, 1992) indica essa dificuldade, que é particularmente frustrante no início do estudo das áreas de pesquisa que utilizam essa terminologia. Segundo van Kampen, o termo master equation foi utilizado pela primeira vez em um artigo de 1940 e tinha por objetivo denominar uma equação, a partir da qual todos os resultados da análise seriam derivados. A partir de então, a utilização do termo convergiu para designar um tipo especial de equação que vincula as variáveis de um sistema estocástico a balanço de probabilidades. O próprio van Kampen, em seu livro define master equation como uma forma equivalente da equação de Chapman-Kolmogorov especializada para processos markovianos. (WISEMAN; MILBURN, 2010), por outro lado, argumentam que as aproximações originalmente utilizadas para tornar markoviana a equação dinâmica geral da evolução das distribuições de probabilidade não são suficientes para atingir o objetivo. Assim, a equação resultante, às vezes denominada equação de Redfield, após as aproximações de Born-Markov (acoplamento fraco entre o sistema e o ambiente, aproximação de Born, e eliminação da dependência explícita do estado do sistema dos instantes anteriores, aproximação de Markov) pode mesmo assim resultar em uma equação não Markoviana, vide (WISEMAN; MILBURN, 2010, pp. 97 e ss.).

Na literatura científica em português encontramos o termo equação mestra, traduzindo diretamente o termo master equation. 


\subsection{Dissipação ad hoc}

Seguimos neste item a exposição de (CARMICHAEL, 1990). Na mecânica clássica, as características essenciais dos processos de dissipação - o decaimento das amplitudes de deslocamento e de velocidade e da energia do sistema - pode ser incorporada no modelo matemático por meio da incorporação no balanço de forças de um termo proporcional à velocidade. Assim, as equações de movimento originais:

$$
\begin{aligned}
& \dot{q}=p \\
& \dot{p}=-q
\end{aligned}
$$

Após a modificação descrita ficam:

$$
\begin{aligned}
& \dot{q}=p \\
& \dot{p}=-q-\gamma p
\end{aligned}
$$

O modelo de dissipação ad hoc baseia-se na transposição de modificações equivalentes às equações de movimento quânticas. O procedimento de quantização usual é dado pela transformação dos pares de variáveis conjugadas clássicas, $q_{i}$ e $p_{i}$, em operadores, $\hat{q}_{i}$ e $\hat{p}_{i}$, para os quais passam a valer as relações de comutação:

$$
\left[\hat{q}_{i}, \hat{p}_{i}\right]=i \hbar
$$

As equações de Heisenberg ficam:

$$
\begin{aligned}
& \dot{\hat{q}}=\hat{p} \\
& \dot{\hat{p}}=-\hat{q}
\end{aligned}
$$

E com a modificação ad hoc passam a se escrever como:

$$
\begin{aligned}
& \dot{\hat{q}}=\hat{p} \\
& \dot{\hat{p}}=-\hat{q}-\gamma \hat{p}
\end{aligned}
$$

Como resultado das equações obtidas, o valor esperado de $\hat{q}_{i}$ e $\hat{p}_{i}$ será amortecido, da mesma forma como ocorre com as variáveis clássicas. Entretanto, ao calcular a variação do comutador $[\hat{q}, \hat{p}]$ verificamos que:

$$
\frac{d}{d t}[\hat{q}, \hat{p}]=\frac{d}{d t}(\hat{q} \hat{p}-\hat{p} \hat{q})=\dot{\hat{q}} \hat{p}+\hat{q} \dot{\hat{p}}-\dot{\hat{p}} \hat{q}-\hat{p} \dot{\hat{q}}
$$

E como consequência:

$$
\frac{d}{d t}[\hat{q}, \hat{p}]=[\dot{\hat{q}}, \hat{p}]+[\hat{q}, \dot{\hat{p}}]
$$


Substituindo os valores de $\dot{\hat{q}}$ e $\dot{\hat{p}}$ obtidos das equações de Heisenberg (4.7) e utilizando as relações de comutação canônicas (4.5):

$$
\frac{d}{d t}[\hat{q}, \hat{p}]=-\gamma[\hat{q}, \hat{p}]
$$

e

$$
[\hat{q}(t), \hat{p}(t)]=\exp (-\gamma t)[\hat{q}(0), \hat{p}(0)]=i \hbar \exp (-\gamma t)
$$

Em face desse amortecimento das relações de comutação, a relação de incerteza de Heisenberg fica:

$$
\Delta q \Delta p=\frac{1}{2} \hbar \exp (-\gamma t)
$$

Obviamente, essa modificação ad hoc introduzida nas equações de movimento quânticas é incompatível com a estrutura interna da teoria. Em muitas aplicações da mecânica quântica não é necessário fazer nenhuma menção a processos dissipativos, no entanto, a óptica quântica, por exemplo, é baseada em dispositivos imperfeitos que estão sujeitos à dissipação. Muitas aplicações tecnológicas baseadas em dispositivos que operam em regime quântico necessitam modelar e tratar os efeitos dissipativos internos. Do ponto de vista da engenharia, a capacidade de obter modelos quantitativos com razoável fidelidade é pré-requisito para se projetar dispositivos confiáveis que forneçam resultados previsíveis e, portanto, tecnologicamente úteis.

De forma a obter uma compreensão adequada dos elementos de irreversibilidade presentes na dinâmica de sistemas quânticos abertos, é importante realizar uma análise mais completa dos fenômenos físicos envolvidos. A dissipação se dá por meio da interação entre o sistema de interesse e seu ambiente, que é um sistema físico grande e complexo. Tendo em vista o teorema da flutuação-dissipação, que também é válido nos domínios da física quântica, é necessário incorporar no modelo do sistema quântico aberto os efeitos decorrentes das flutuações térmicas do ambiente.

A consideração dos processos de flutuação é fundamental para a solução do problema de dissipação quântico. Apenas após sua inclusão é que as relações canônicas de comutação restam preservadas (CARMICHAEL, 1990).

Na interpretação física dada por (GARDINER; ZOLLER, 2004, cap. 1), as flutuações elétricas estudadas por Nyquist e Johnson são inerentes à mecânica estatística. É por meio dessas flutuações dinâmicas que a distribuição de Boltzmann é preservada nas variáveis canônicas apropriadas à descrição de um circuito elétrico.

Quanticamente, as equações de dissipação podem ser derivadas de forma a preservar as relações de comutação da mecânica quântica apenas se a interação do sistema com o ambiente for considerada. Para o caso simplificado de dois osciladores acoplados entre si, pode-se mostrar que as relações de comutação são preservadas. 
O acoplamento de dois osciladores quânticos pode ser descrito por um Hamiltoniano do tipo

$$
H=\hbar \omega a^{\dagger} a+\hbar \omega b^{\dagger} b+\hbar \kappa\left(a^{\dagger} b+a b^{\dagger}\right)
$$

É importante observar que uma aproximação do tipo rotating-wave está sendo utilizada. Por meio dessa aproximação, são desprezados termos do tipo $a b$ e $a^{\dagger} b^{\dagger}$, que estão associados a frequências oscilatórias consideravelmente maiores e são não conservativos, vide, por exemplo, (WISEMAN; MILBURN, 2010; CARMICHAEL, 1990). ${ }^{5}$

Para os osciladores, quando desacoplados, valem as relações de comutação canônicas típicas

$$
\left[a, a^{\dagger}\right]=1, \quad\left[b+b^{\dagger}\right]=1
$$

A solução da equação (4.13) é

$$
\begin{aligned}
& a(t)=e^{-i \omega t}[a(0) \cos k t-i b(0) \sin k t] \\
& b(t)=e^{-i \omega t}[b(0) \cos k t-i a(0) \sin k t]
\end{aligned}
$$

de onde decorre que

$$
\left[a(t), a^{\dagger}(t)\right]=\left[a(0), a^{\dagger}(0)\right] \cos ^{2} k t+\left[b(0), b^{\dagger}(0)\right] \operatorname{sen}^{2} k t=1 .
$$

ou seja, as relações de comutação canônicas do sistema de interesse são preservadas. ${ }^{6}$

Este é, portanto, um ponto de partida viável para se modelar quanticamente a dissipação de energia de um sistema aberto. O modelo apresentado em (CARMICHAEL, 1990) é por sua vez baseado no modelo desenvolvido por Senitzky e usado para descrever perdas em cavidades ressonantes de maser. Conforme indicações do autor, pontos de vista alternativos podem ser encontrados em Caldeira e Leggett e em Ray, vide referências em (CARMICHAEL, 1990). É interessante notar que a preservação das relações de comutação canônicas ocorre pela mistura dos osciladores, que foram mesclados de tal forma que as relações canônicas puderam ser preservadas. De forma reiterada e em um nível de complexidade maior, o acoplamento sistemático da dinâmica dos osciladores é o mesmo fenômeno que ocorre nos modelos de dissipação de energia em sistemas quânticos.

5 A aproximação rotating-wave é justificada porque há escalas de diferentes ordens envolvidas no modelo. Despreza-se a escala das oscilações mais rápidas porque sua contribuição na dinâmica da escala mais lenta é nula, na média, vide (WISEMAN; MILBURN, 2010, p. 104). Vide (CARMICHAEL, 1990, p. 17) para uma discussão das limitações dessa aproximação e (WISEMAN; MILBURN, 2010, pp. 109 e ss.) para uma discussão sobre dinâmica irreversível sem a aproximação rotating-wave.

6 Deve-se notar que no acoplamento entre os osciladores descrito acima, ainda não ocorre dissipação de energia, no entanto, a apreservação das relações de comutação é um importante resultado. Além disso, ocorre um fluxo de energia bidirecional e equivalente entre os osciladores, o que aponta para uma analogia com os fenômenos de dissipação e flutuação. 


\subsection{Equação de Redfield e Equações Mestras}

A equação de Redfield modela a interação de um sistema quântico com seu meio ambiente, assumindo determinadas e realizando algumas simplicações, como será mostrado a seguir. O modelo parte de uma perspectiva geral, a partir da qual existe um sistema que, para efeitos práticos pode ser separado em duas partes, o sistema de interesse e o meio ambiente. Ambas as partes são modeladas a princípio e cada uma está associada a um espaço de estados, que são compostos por produto vetorial para definir o espaço de estados do sistema + meio ambiente. O sistema que representa o meio ambiente pode assumir várias formas diferentes, assim a parte que o modela em uma equação de dissipação deve possuir uma estrutura bastante geral. Conforme mostramos na seção anterior, não se pode prescindir de incorporar um modelo do meio ambiente que interage com o sistema. A incorporação de termos ad hoc às equações de movimento leva a modelos que não mantêm a consistência das equações da mecãnica quântica. Iniciamos com a definição do Hamiltoniano em uma forma geral dado por, vide equação 4.2:

$$
H=H_{S}+H_{R}+H_{S R}
$$

Seja $\chi(t)$ o operador densidade do sistema em $S \otimes R$. A equação dinâmica exata é dada por:

$$
\dot{\chi}=\frac{1}{i \hbar}[H, \chi] .
$$

O operador densidade do sistema restrito ao sistema de interesse $S$ é:

$$
\rho(t) \equiv \operatorname{tr}_{R}[\chi(t)]
$$

onde $t r_{R}$ é a operação de traço parcial.

Na perspectiva de interação, a partir da qual deixaremos implícita a dependência da dinâmica do sistema aos termos $H_{S}$ e $H_{R}$ do Hamiltoniano, tem-se que:

$$
\widetilde{\chi}(t) \equiv e^{\frac{i}{\hbar}\left(H_{S}+H_{R}\right) t} \chi(t) e^{-\frac{i}{\hbar}\left(H_{S}+H_{R}\right) t},
$$

então,

$$
\begin{gathered}
\dot{\tilde{\chi}}(t)=\frac{1}{i \hbar}\left[\widetilde{H}_{S R}(t), \widetilde{\chi}(t)\right] \\
\widetilde{H}_{S R}(t)=e^{\frac{i}{\hbar}\left(H_{S}+H_{R}\right) t} H_{S R} e^{-\frac{i}{\hbar}\left(H_{S}+H_{R}\right) t}
\end{gathered}
$$

integrando (4.16)

$$
\widetilde{\chi}(t)=\chi(0)+\frac{1}{i \hbar} \int_{0}^{t} d t^{\prime}\left[\widetilde{H}_{S R}\left(t^{\prime}\right), \widetilde{\chi}\left(t^{\prime}\right)\right.
$$

e substituindo de volta em (4.16), chegamos a:

$$
\left.\dot{\tilde{\chi}}=\frac{1}{i \hbar}\left[\widetilde{H}_{S R}(t), \chi(0)\right]-\frac{1}{\hbar^{2}} \int_{0}^{t} d t^{\prime}\left[\widetilde{H}_{S R}^{(} t\right),\left[\widetilde{H}_{S R}\left(t^{\prime}\right), \widetilde{\chi}\left(t^{\prime}\right)\right]\right]
$$


que é uma equação integro-diferencial exata. A restrição da equação (4.17) ao sistema é dada por:

$$
\dot{\tilde{\rho}}=-\frac{1}{\hbar^{2}} \int_{0}^{t} d t^{\prime} \operatorname{Tr}_{R}\left\{\left[\widetilde{H}_{S R}(t),\left[\widetilde{H}_{S R}(t), \widetilde{\chi}\left(t^{\prime}\right)\right]\right]\right\},
$$

em que se assume a hipótese de que o primeiro termo da equação pode ser incorporada ao Hamiltoniano do sistema por uma transformação conveniente, vide (JACOBS, 2014).

Assume-se a hipótese de que o estado inicial de $S \otimes R$ é um estado sem emaranhamento, portanto:

$$
\tilde{\chi}(0)=\rho_{S}(0) \otimes \rho_{R}(0) .
$$

Pela aproximação de Born, a dinâmica do sistema é desprezível com relação à dinâmica do ambiente, pelo fraco acoplamento, assim, podemos escrever que a evolução do operador densidade $\chi$ mantém a forma produto:

$$
\tilde{\chi}(t)=\rho_{S}(t) \otimes \rho_{R}(0) .
$$

Com a aproximação de Born, não se quer dizer que o estado do sistema + ambiente será sempre um estado produto. De fato, com a evolução do sistema total, é esperado que o sistema fique acoplado - emaranhado - ao ambiente ou reservatório. No entanto, tratase de uma hipótese razoável para derivar a equação dinâmica dissipativa das variáveis de estado do sistema, vide (WISEMAN; MILBURN, 2010). ${ }^{7}$

Pela aproximação de Markov, temos que

$$
\rho_{S}\left(t^{\prime}\right) \otimes \rho_{R}(0)=\rho_{S}(t) \otimes \rho_{R}(0) \text {. }
$$

E podemos encontrar a equação resultante em duas formas

$$
\dot{\widetilde{\rho}}=-\frac{1}{\hbar^{2}} \int_{0}^{t} d t^{\prime} \operatorname{Tr}_{R}\left\{\left[\widetilde{H}_{S R}\left(t^{\prime}\right),\left[\widetilde{H}_{S R}\left(t^{\prime}\right), \rho(t) \otimes \rho_{R}(0)\right]\right]\right\}
$$

$\mathrm{ou}$

$$
\dot{\tilde{\rho}}=\frac{1}{i \hbar}\left[\widetilde{H}_{S R}(t), \rho(t)\right]+-\frac{1}{\hbar^{2}} \int_{0}^{t} d t^{\prime} \operatorname{Tr}_{R}\left\{\left[\widetilde{H}_{S R}\left(t^{\prime}\right),\left[\widetilde{H}_{S R}\left(t^{\prime}\right), \rho(t) \otimes \rho_{R}(0)\right]\right]\right\}
$$

Essa é a chamada equação de Redfield. De acordo com (WISEMAN; MILBURN, 2010), a equação é Markoviana apenas se passarmos o limite inferior da integral para $-\infty$ tornando ainda mais estrita a aproximação de Markov:

$$
\dot{\tilde{\rho}}=\frac{1}{i \hbar}\left[\widetilde{H}_{S R}(t), \rho(t)\right]+-\frac{1}{\hbar^{2}} \int_{-\infty}^{t} d t^{\prime} \operatorname{Tr}_{R}\left\{\left[\widetilde{H}_{S R}\left(t^{\prime}\right),\left[\widetilde{H}_{S R}\left(t^{\prime}\right), \rho(t) \otimes \rho_{R}(0)\right]\right]\right\}
$$

A equação resultante é denominada equação mestra para o sistema quântico aberto na aproximação de Born-Markov e pode ser utilizada para modelar com boa aproximação sistemas quânticos abertos, resultando em uma equação diferencial autônoma.

$7 \quad$ Importante notar, neste ponto, que estruturalmente a equação de movimento do sistema aberto está de fato assumindo uma forma produto, portanto não emaranhada, para o estado do sistema global. De todo modo a dinâmica do estado $\rho_{S} \otimes \rho_{R}$ a partir do Hamiltoniano de interação, causará em geral emaranhamento. 
Apresentamos a seguir alguns exemplos de aplicação da equação mestra 4.18 na obtenção de equações específicas para alguns modelos simples e úteis. Não será apresentada, nos exemplos, a dedução das equações que poderá ser encontrada, e.g. em (WISEMAN; MILBURN, 2010; CARMICHAEL, 1991; CARMICHAEL, 1990).

A dinâmica que modela o fenômeno da emissão espontânea de radiação por um átomo é apresentada no primeiro exemplo abaixo. Tanto neste exemplo quanto nos seguintes, a aproximação rotating-wave é realizada. ${ }^{8}$

Exemplo 43 (Emissão espontânea de radiação). No caso de um átomo na presença de um campo em estado de vácuo, pode-se modelar a emissão espontânea de radiação levando os átomos de um estado excitado para um estado não excitado. O átomo constitui, portanto, um sistema de dois níveis. ${ }^{9}$

$$
\dot{\rho}=-i \frac{\Delta \omega a}{2}\left[\sigma_{z}, \rho\right]+\gamma \mathcal{D}\left[\sigma_{-}\right] \rho
$$

$\Delta \omega_{a}$ é desvio de frequência de Lamb, $\gamma$ é taxa de decaimento por radiação e $\mathcal{D}$ é um superoperador típico da teoria de sistemas dinâmicos quânticos abertos que modela dinamicamente a interação com o meio ambiente e a dissipação, sendo dado por:

$$
\mathcal{D}[A] \rho \equiv A \rho A^{\dagger}-\frac{1}{2}\left(A^{\dagger} A \rho+\rho A A^{\dagger}\right)
$$

É interessante notar no modelo acima que, a partir da aplicação da teoria desenvolvida acima para estudar os efeitos de um acoplamento fraco entre um sistema quântico de interesse e seu meio ambiente, é possível representar sistemas físicos que apresentam dissipação. No modelo acima, a dissipação é representada pela taxa de decaimento radiativo. Assim, um conjunto de átomos inicialmente excitados, representado por um operador densidade $\rho_{0}$, perde energia por emissão de radiação até que ao final reste o mesmo conjunto de átomos inicial no estado final não excitado.

O desvio de frequência de Lamb, $\Delta \omega_{a}$, apresenta em geral um valor muito baixo, vide (WISEMAN; MILBURN, 2010).

Um segundo exemplo, mostra o comportamento de átomo na presença de um campo eletromagnético clássico de excitação constituído por um laser.

8 Alguns exemplos sobre dissipação em sistemas quânticos abertos que dispensam o uso da aproximação rotating-wave podem ser encontrados em (WISEMAN; MILBURN, 2010).

9 Em teoria quântica de campos, o estado de vácuo não é um estado de energia nula, o desvio de frequência de Lamb, explícito no modelo abaixo, é devido ao acoplamento do átomo com o estado de vácuo e pode ser propriamente deduzido apenas pela utilização de mecânica quântica relativística e de teoria da renormalização, vide por exemplo (WISEMAN; MILBURN, 2010). Na prática, é um valor muito baixo. 
Exemplo 44 (Interação entre um átomo de dois níveis e um laser). Ainda na presenção do vácuo como meio ambiente externo, pode-se adicionar um termo no Hamiltoniano para modelar a presença de um campo eletromagnético coerente, ou seja, um laser. A adição do termo diretamente na equação resultante é justificada quando o Hamiltoniano que descreve o campo coerente externo é pequeno em comparação ao Hamiltoniano principal do sistema, que modela os dois níveis de energia. Assim, o Hamiltoniano adicionado tem efeito desprezível na dedução da equação dinâmica do sistema a partir da equação de Redfield e, portanto, pode ser adicionado ao final, vide (WISEMAN; MILBURN, 2010).

$$
H_{\text {drive }}=\frac{\Omega}{2} \sigma_{x}+\frac{\Delta}{2} \sigma_{z}
$$

$\Delta=\omega_{a}+\Delta \omega_{a}-\omega_{0}$ é o detuning ou desvio de frequência, é a diferença entre a frequência do laser e a frequência de ressonância real do modo de excitação do átomo e $\Omega$ é a frequência de Rabi, proporcional à amplitude da radiação incidente e ao momento de dipolo atômico.

E equação dinâmica do sistema é dada por

$$
\dot{\rho}=-i\left[\frac{\Delta}{2} \sigma_{z}+\frac{\Omega}{2} \sigma_{x}, \rho\right]+\gamma \mathcal{D}\left[\sigma_{-}\right] \rho
$$

Importante notar que se os dois parâmetros acima, $\Omega$ e $\Delta$, forem manipuláveis livremente em uma faixa de valores determinada, o estado quântico do átomo, representado como um elemento do grupo $S U(2)$, seria controlável, tendo em vista a teoria de controlabilidade do capítulo anterior, pois a álgebra de Lie gerada pela álgebra dinâmica, $\left\{\sigma_{x}, \sigma_{z}\right\}$, é a álgebra $s u(2)=\left\{\sigma_{x}, \sigma_{y}, \sigma_{z}\right\} .{ }^{10}$

As equações de Bloch para o sistema descrito acima são (WISEMAN; MILBURN, 2010, p. 107):

$$
\begin{array}{rrrr}
\dot{x} & =-\frac{\gamma}{2} x & -\Delta y & \\
\dot{y}= & \Delta x & -\frac{\gamma}{2} y & -\Omega z \\
\dot{z}= & & \Omega y & -\gamma(z+1)
\end{array}
$$

A dinâmica do sistema bilinear representada acima está associada a um campo livre dissipativo, $A_{0}$, e a campos de controle que induzem rotações na esfera de Bloch, $A_{x}$

$\overline{10}$ Interessante notar que, se o parâmetro $\Delta$ puder ser manipulado livremente, estaríamos diante de um VCO óptico, de fato, isso é o que parece ocorrer nos dispositivos denominados moduladores eletro-ópticos. 
e $A_{z}$, parametrizados nos controles $\Omega$ e $\Delta$ respectivamente.

$$
A_{0}=\left(\begin{array}{ccc}
-\frac{\gamma}{2} & 0 & 0 \\
0 & -\frac{\gamma}{2} & 0 \\
0 & 0 & -\gamma
\end{array}\right), \quad A_{x}=\left(\begin{array}{ccc}
0 & 0 & 0 \\
0 & 0 & -1 \\
0 & -1 & 0
\end{array}\right), \quad A_{z}=\left(\begin{array}{ccc}
0 & -1 & 0 \\
1 & 0 & 0 \\
0 & 0 & 0
\end{array}\right)
$$

As matrizes $A_{x}$ e $A_{z}$ são capazes de gerar por comutação a álgebra so(3), pela qual

$$
\left[A_{x}, A_{y}\right]=A_{z} \quad, \quad\left[A_{y}, A_{z}\right]=A_{x} \quad, \quad\left[A_{z}, A_{x}\right]=A_{y}
$$

A álgebra acima é capaz de gerar todo o grupo das rotações em $\mathbb{R}^{3}, S O(3)$, portanto, a projeção de um estado qualquer $\bar{\rho}$ na superfície da esfera de Bloch pode ser livremente controlada, vide (D'ALESSANDRO, 2008, pp. 125 e ss.).

A hipótese de que o sistema se encontra em interação com um ambiente no estado de vácuo pode ser levantada. Assim, caso o ambiente esteja em um estado de equilíbrio térmico, a equação dinâmica do sistema será:

$$
\dot{\rho}=-i\left[\frac{\Delta}{2} \sigma_{z}+\frac{\Omega}{2} \sigma_{x}, \rho\right]+\gamma(\bar{n}+1) \mathcal{D}\left[\sigma_{-}\right] \rho+\gamma \bar{n} \mathcal{D}\left[\sigma_{+}\right] \rho
$$

em que

$$
\bar{n}=\frac{1}{e^{\frac{\hbar \omega a}{k_{B} T}}-1}
$$

é o número de fótons no estado térmico dado por uma temperatura $T$, ou seja, calculados a partir da distribuição de Planck.

O modo de vibração de um campo eletromagnético em uma cavidade óptica está também sujeito a amortecimento radiativo. Em óptica quântica, o termo cavidade se refere a qualquer estrutura, feita tipicamente de materiais dielétricos, capaz de armazenar energia eletromagnética em níveis discretos. Uma cavidade pode ser construída com um par de espelhos convexos face a face com seus eixos alinhados. A perda de radição se dá porque os espelhos não são perfeitos e há fuga de radiação por transmissão. O caso mais simples a ser analisado é dado por um par de espelhos, quando um é considerado perfeito e outro possui perda.

Exemplo 45 (Radiação em cavidade óptica). Sejam $a$ e $a^{\dagger}$ os operadores de criação e aniquilação para o modo da cavidade formada pelos espelhos e $b_{k}$ e $b_{k}^{\dagger}$ os operadores de criação e aniquilação para os modos do ambiente. 
O Hamiltoniano do sistema composto, na aproximação rotating-wave é dado por:

$$
H=\omega_{c} a^{\dagger} a+\sum_{\kappa} \omega_{\kappa} b_{\kappa}^{\dagger} b_{\kappa}+\sum_{\kappa} g_{\kappa}\left(a^{\dagger} b_{\kappa}+a b_{\kappa}^{\dagger}\right)
$$

e a dinâmica do sistema é dada por

$$
\dot{\rho}=\gamma(\bar{n}+1) \mathcal{D}[a] \rho+\gamma \bar{n} \mathcal{D}\left[a^{\dagger}\right] \rho
$$

O número médio de fótons na cavidade $\left\langle a^{\dagger} a\right\rangle$ é dado por

$$
\frac{d}{d t}\left\langle a^{\dagger} a\right\rangle=-\gamma\left\langle a^{\dagger} a\right\rangle+\gamma \bar{n}
$$

E o número médio de fótons no estado de equilíbrio é, como se deveria esperar, vide (WISEMAN; MILBURN, 2010, p. 108), (CARMICHAEL, 1990, p. 18):

$$
\left\langle a^{\dagger} a\right\rangle=\bar{n}
$$

Ao resgatar a dinâmica livre do sistema por uma mudança de coordenadas, ficamos diante da equação mestra do oscilador harmônico amortecido:

$$
\dot{\rho}=-i \omega\left[a^{\dagger} a, \rho\right]+\gamma(\bar{n}+1) \mathcal{D}[a] \rho+\gamma \bar{n} \mathcal{D}\left[a^{\dagger}\right] \rho
$$

A evolução de um sistema modelado por um oscilador harmônico amortecido também pode ser analisada por meio da dinâmica esperada para a média dos observáveis posição e momento linear, $\langle x\rangle$ e $\langle p\rangle$. No caso do ambiente em estado de vácuo, ou seja, para $T=0$, obtemos as seguintes equações, vide (JACOBS, 2014, p. 200):

$$
\begin{array}{lr}
\frac{d}{d t}\langle x\rangle=-\frac{\gamma}{2}\langle x\rangle \quad+\langle p\rangle \\
\frac{d}{d t}\langle p\rangle=-\langle x\rangle \quad-\frac{\gamma}{2}\langle p\rangle
\end{array}
$$

Na equação 4.20 acima, notamos que, à forma familiar das equações de movimento para as médias das variáveis canônicas dadas pelo teorema de Ehrenfest, vide (SAKURAI, 1994, pp. 86 e 87), foram adicionadas duas parcelas associadas ao amortecimento, divididas entre as variáveis de estado $x$ e $p$.

A dinâmica acima também pode ser modificada para incluir a excitação por um laser de frequência $\omega_{c}$ por

$$
H_{\text {drive }}=i\left(\epsilon a^{\dagger}-\epsilon^{*} a\right)
$$

Este é o início do campo de pesquisa da óptica quântica. Para um tratamento do assunto é possível consultar (WALLS; MILBURN, 2008; SCULLY; ZUBAIRY, 1997). Os 
casos exemplificados abaixo descrevem tanto o caso de uma cavidade óptica guiada ( $d r i$ ven) pelo ruído eletromagnético e emitindo ruído composto pelo mesmo tipo de radiação. Mais interessante é o caso de uma fonte de radiação que não se encontra em equilíbrio térmico, por exemplo, um laser. Em ambos os casos, a estatística da distribuição dos fótons que constituem o ruído térmico em uma determinada frequência é obtida da distribuição de Planck para o caso de equilíbrio térmico. É interessante notar que, na faixa de temperatura ambiente, em um laboratório e nas frequência ópticas em geral utilizadas, o número médio de fótons é dado por $\bar{n}=10^{-42}$, ou seja, oticamente são completamente desprezíveis. No entanto, na faixa de microondas, mesmo em hélio líquido, alguns fotons estarão presentes no ambiente em equilíbrio térmico, vide (CARMICHAEL, 1991). Essas observações são importantes, pois tanto circuitos ópticos quanto circuitos eletrônicos com supercondutores formando junções de Josephson e operando na faixa de microondas podem ser usados como elementos principais em duas importantes arquiteturas de processamento quântico de informação. É interessante ressaltar que ambas são modeladas por um Hamiltoniano que possui a mesma estrutura, por isso recebem denominações parecidas, cavity quantum electrodynamics, cavity QED, e circuit quantum electrodynamics, circuit QED, vide (GIRVIN, 2014). Tal Hamiltoniano é associado ao famoso modelo de Jaynes-Cummings.

Em uma analogia à teoria de amortecimento de um oscilador harmônico clássico, associada tanto a um termo dissipativo quanto a um termo de flutuação, a teoria desenvolvida até o momento já obteve sucesso na incorporação dos termos dissipativos. Ainda falta uma adição ao modelo acima relacionada aos termos de ruído ou flutuação. As análises realizadas acima foram pautadas em equações mestras e evolução dinâmica das médias de variáveis canônicos. Esses dois instrumentos ao modelarem variáveis estocásticas eliminam o ruído, pois modelam apenas o comportamento médio da variável. Assim, uma forma intuitiva e simples de ampliar a modelagem de sistemas quânticos abertos para tratar diretamente o ruído é dada pela ampliação das equações dinâmicas acima para incluir e tratar diretamente processos estocásticos.

Mesmo classicamente, a teoria de dissipação é completada pela incorporação dos fenômenos de flutuação de forma a resgatar uma compatibilidade com a mecânica estatística.

Um oscilador clássico sujeito a dissipação e flutuação é representado por uma equação de Langevin dada por, vide (JACOBS, 2014, p. 189):

$$
\begin{aligned}
d \bar{x} & =\frac{\bar{p}}{m} d t \\
d \bar{p} & =-V^{\prime}(\bar{x}) d t-\gamma \bar{p} d t+\sqrt{\gamma m \kappa T} d W .
\end{aligned}
$$

O terceito termo modela a flutuação e está associada a $d W$, que é um incremento de processo de Wiener. Fisicamente modela uma força de natureza estocástica com distribuição 
gaussiana.

Por uma mudança de coordenadas

$$
x=\frac{\bar{x}}{\sqrt{2 m \omega / \hbar}}, \quad p=\frac{\bar{p}}{\sqrt{\hbar m \omega / 2}} .
$$

Ficamos com um Hamiltoniano

$$
H=\hbar \omega\left(\frac{x^{2}}{4}+\frac{p^{2}}{4}\right)
$$

e com respectivas equações de movimento

$$
\begin{aligned}
& d x=\omega p d t \\
& d p=-\omega x d t-\gamma p d t+\sqrt{4 \gamma n_{T}} d W
\end{aligned}
$$

em que $n_{T}=\frac{\kappa T}{2 \hbar \omega}$ é a energia média de um oscilador em equilíbrio com o meio ambiente térmico a uma temperatura $T$.

Quando o amortecimento $\gamma$ é baixo em comparação à frequência $\omega$ as equações (4.24) possuem uma forma equivalente, que é simétrica

$$
\begin{aligned}
& d x=\omega p d t-\frac{\gamma}{2} x d t+\sqrt{2 \gamma n_{T}} d W_{1} \\
& d p=-\omega x d t-\frac{\gamma}{2} p d t+\sqrt{2 \gamma n_{T}} d W_{2}
\end{aligned}
$$

em que $d W_{1}$ e $d W_{2}$ são dois incrementos de Wiener distintos.

A forma (4.25) é possível porque, devido ao baixo amortecimento, a oscilação linear carrega tanto o amortecimento quando a flutuação de uma variável $p$ outra. $p \rightarrow$ $\dot{x} \rightarrow x \rightarrow \dot{p} \rightarrow p \ldots$. Para um oscilador quântico uma forma simétrica equivalente à forma das equações (4.25) é que ocorre.

Esta seção tem por objetivo apenas motivar fisicamente as equações mestras, pois grande parte, possivelmente toda, a dinâmica determinística de sistemas quânticos pode ser modelada usando essa ferramenta. Colocando o tratamento apresentado em perspectiva, as equações mestras modelam um sistema físico pela perspectiva de Schrödinger, utilizando sistemas de coordenadas que simplificam as equações dinâmicas por eliminarem frequências estacionárias, essa é a causa da introdução da perspectiva de interação, interaction picture.

Escolhemos para ilustrar a teoria o problema da modelagem a dinâmica de dissipação da energia em sistemas quânticos, o que nos levou de encontro ao problema de manter a consistência da teoria de dissipação com as relações de comutação da mecânica quântica. No entanto, essa não é a única questão envolvida na busca por consistência em uma teoria estocástica aplicável aos sistemas quânticos, vide (GARDINER; ZOLLER, 2004, pp. 10 e ss.). Além da consistência com a mecânica quântica, por meio da preservação 
das relações de comutação canônicas, uma teoria de sistemas estocásticos aplicável aos sistemas quânticos deve também manter-se em consistência com a mecânica estatística.

Em uma linha de transmissão de radiação eletromagnética que tenha comprimento finito, as ondas podem sofrer reflexão nas extremidades. Assim, em uma linha de transmissão ideal, composta apenas de elementos reativos, de comprimento finito a energia eletromagnética encontra-se confinada, considerando que a reflexão da onda nas extremidades ocorre perfeitamente, sem perdas. No entanto, uma linha de transmissão semiinfinita pode dissipar energia, pois uma onda lançada em sua extremidade carrega energia e não mais retorna. Na descrição oferecida por Girvin, (GIRVIN, 2014, p. 222), como é comum na análise de processos dissipativos, existe uma sutileza associada à ordem dos limites. Tanto o comprimento quanto o tempo tendem ao infinito. Se o tempo for a infinito mais rápido que o comprimento da linha de transmissão, tem-se como resultado que não ocorre dissipação. Na ordem contrária dos limites, ocorre a dissipação de energia.

A partir de uma perspectiva mais geral, aplicável a diversas situações físicas, um sistema em contato com o reservatório com que troca energia apresentará dissipação de energia se o reservatório em equilíbrio térmico apresentar um número infinito de graus de liberdade de tal forma que os modos característicos do reservatório formem um continuum de frequências, vide (BREUER; PETRUCCIONE, 2002, pp. 115 e ss.)

\subsubsection{Forma de Lindblad}

Apresentamos a seguir a forma de Lindblad, ou forma de Gorini-KossakowskiSudarshan (GORINI; KOSSAKOWSKI; SUDARSHAN, 1976) apud (EDWARDS; BELAVKIN, 2005), para equações mestras markovianas. Demos importância à relação entre a estrutura da equação e a teoria de semigrupos, que mantém relação com sistemas dissipativos. A forma de Lindblad mantém uma relação próxima com a teoria de operações e efeitos a ser apresentada abaixo, pois ambas buscam apresentar as estruturas matemáticas de equações de evolução que mantêm invariantes determinadas propriedades essenciais de um modelo quântico. E ambas apresentam seus resultados na forma de superoperadores.

A solução de uma equação mestra define, $\forall t \geq 0$, um mapa

$$
\begin{aligned}
& \mathcal{N}_{t}: \rho(0) \rightarrow \rho(t) \\
& \rho(t)=\mathcal{N}_{t} \rho(0)
\end{aligned}
$$

O mapa $\mathcal{N}_{t}$ deve ser completamente positivo $\forall t$ o que significa que, se $\mathcal{N}$ está definido para um sistema $S$ e houver uma ampliação de $S$ para $S \otimes E, \mathcal{N}$ continua sendo positivo em $S$, ou seja, $(\mathcal{N} \otimes \mathcal{I})_{t}$ é positivo, em que $\mathcal{I}$ é a identidade em $E$.

Um semigrupo dinâmico quântico é uma família de mapas completamente positivos $\mathcal{N}_{t}$, para $t \geq 0$, tal que 
$1-\mathcal{N}_{t} \mathcal{N}_{s}=\mathcal{N}_{t+s}$

2 - $\operatorname{Tr}\left[\left(\mathcal{N}_{t} \rho\right) A\right]$ é uma função contínua em $t$ para qualquer operador densidade $\rho$ e qualquer operador auto adjunto $A$.

Trata-se de um semigrupo, pois a inversa não existe necessariamente, $\mathcal{N}_{t}$ não está definido para $t<0$. Essas condições formais definem uma dinâmica markoviana. Dado um semigrupo $\mathcal{N}_{t}$, pode-se mostrar que $\exists \mathcal{L}$, um superoperador, tal que

$$
\text { se } \rho(t)=\mathcal{N}_{t} \rho(0), \quad \text { então } \quad \dot{\rho}(t)=\mathcal{L} \rho(t), \quad \text { ou seja }
$$

$\rho(t)=e^{\mathcal{L} t} \rho(0)$, além disso, o superoperador $\mathcal{L}$ deve ter a forma de Lindblad

$$
\begin{gathered}
\mathcal{L} \rho=-i[H, \rho]+\sum_{\kappa} \mathcal{D}\left[L_{\kappa}\right] \rho \\
\mathcal{D}\left[A \left[\rho \equiv A \rho A^{\dagger}-\frac{1}{2}\left(A^{\dagger} A \rho+\rho A^{\dagger} A\right) .\right.\right.
\end{gathered}
$$

Assim, a dinâmica de um sistema quântico aberto é markoviana se, e somente se, sua equação mestra apresentar a forma de Lindblad.

Observação 46. A título ilustrativo e com o objetivo de acrescentar mais um elemento ao paralelo desenvolvido entre os sitemas clássicos e quânticos, apresentamos a seguir um modelo de sistema quântico estocástico, que incorpora estrutura adicional para lidar diretamente com flutuação e ruído. Para um operador $\hat{s}$ a equação de movimento na perspectiva de Heisenberg é dada por:

$$
\begin{aligned}
d \hat{s}= & d t\left(\hat{c}^{\dagger} \hat{s} \hat{c}-\frac{1}{2}\left\{\hat{c}^{\dagger} \hat{c}, \hat{s}\right\}+i[\hat{H}, \hat{s}]\right) \\
& -\left[d \hat{B}_{i n}^{\dagger}(t) \hat{c}-\hat{c}^{\dagger} d \hat{B}_{i n}(t), \hat{s}\right] .
\end{aligned}
$$

Os elementos associados ao ruído, $d \hat{B}_{i n}$ e $d B_{i n}^{\dagger}$, são operadores não comutativos e podem representar o ambiente em estado de vácuo, equilíbrio térmico e outros estados de fontes de radiação. A equação (4.26) é válida para o ambiente em estado de vácuo, vide (WISEMAN; MILBURN, 2010, p. 144). As equações de movimento na perspectiva de Heisenberg que incorporam ruído são muitas vezes denominadas equações de Langevin quânticas. São em geral baseados na teoria de input-output, vide e.g. (GARDINER; PARKINS; ZOLLER, 1992; JACOBS, 2014; WISEMAN; MILBURN, 2010).

Como, no cálculo estocástico quântico, os termos quadráticos em $d B_{i n}$ e $d B_{i n}^{\dagger}$ são proporcionais a $d t$ nulos. Seus valores em modelos específicos definem uma determinada estatística para o ambiente de um sistema quântico, vide (WISEMAN; MILBURN, 2010, pp. 142-146). 
A partir da equação (4.26) é possível deduzir uma equação mestra que modela o comportamento médio do sistema estocástico

$$
\dot{\rho}=-i[\hat{H}, \rho]+\mathcal{D}[\hat{C}] \rho
$$

que é uma equação mestra na forma de Lindblad. Portanto, os elementos apresentados de uma teoria quântica de ruído possuem auto consistência.

Uma análise mais pormenorizada da teoria da medição quântica especialmente a teoria de medições contínuas poderá mostrar que um dos papeis desenpenhado pelo ruído quântico é o de preservar as relações de comutação e, portanto, o princípio da incerteza.

Cada operador $L_{\kappa}$ na equação de Lindblad representa um canal de irreversibilidade, e os operadores $L_{\kappa}$ podem ser quaisquer. No entanto, a forma de Lindblad não é única.

Mesmo que $\hat{1}, \hat{L}_{1} \ldots \hat{L}_{\kappa}$ sejam linearmente independentes, se $T_{\kappa \ell}$ for uma matriz unitária $K \times K$ a transformação

$$
\hat{L}_{\kappa} \rightarrow \sum_{\ell=1}^{\kappa} T_{\kappa \ell} \hat{L}_{\ell}
$$

mantém a equação mestra invariante. Assim como a transformação

$$
\begin{gathered}
\hat{L}_{\kappa} \rightarrow L_{\kappa}+\chi_{\kappa}, \text { acompanhada de } \\
\hat{H} \rightarrow H-\frac{i}{2} \sum_{\ell=1}^{\kappa}\left(\chi_{\kappa}^{*} \hat{L}_{\kappa}-\chi_{\kappa} L_{\kappa}^{\dagger}\right) .
\end{gathered}
$$

Deve-se ter em mente que o principal e mais importante da forma de Lindblad é que ela preserva a validade de um estado quântico ao modelar as irreversibilidades.

Sobre a não inversibilidade da dinâmica definida por $\mathcal{N}_{t}$, é interessante resgatar uma observação de Preskill (PRESKILL, 1997, cap. 3 pg. 32). O efeito da irreversibilidade observado geometricamente na esfera de Bloch é equivalente a uma contração. É certo que é possível definir uma aplicação na esfera que reverta essa contração, no entanto, uma tal aplicação não pode pertencer a nenhum semigrupo pois não é positiva. Uma dinâmica não conservativa pode realizar a contração, mas nenhum processo físico pode desfazê-la. Um semigrupo evoluindo no sentido inverso do tempo não é um semigrupo.

Assim como uma equação mestra descreve um sistema pela perspectiva de Schrödinger é possível modelar um sistema quântico aberto pela perspectiva de Heisenberg. A equação dinâmica assim obtida é chamada equação mestra adjunta. Para o caso mais simples o superoperador de Lindblad não depende do tempo e a equação adjunta pode ser escrita explicitamente, vide (BREUER; PETRUCCIONE, 2002, p. 120):

$$
\frac{d}{d t} A_{H}=i\left[H, A_{H}\right]+\sum_{\kappa}\left(L_{\kappa}^{\dagger} A_{H} L_{\kappa}-\frac{1}{2} A_{H} L_{\kappa}^{\dagger} K_{\kappa}-\frac{1}{2} L_{\kappa}^{\dagger} L_{\kappa} A_{H}\right)
$$




\subsection{Decoerência}

A dinâmica de sistemas quânticos abertos nos leva a observar dois fenômenos principais distintos entre si e independentes, dissipação e decoerência. A dissipação está relacionada à troca de energia entre os modos de vibração do sistema e os modos do meio. Já a decoerência está relacionada a uma modificação da fase relativa dos modos de vibração do sistema entre as partículas que o compõem e constituem um estado $\rho$. A decoerência é um fenômeno que se apresenta com maior sutileza e apresenta uma forte relação com os processos de medição.

De uma forma mais simples podemos dizer que a decoerência é um processo que tem por efeito destruir superposições de estados quânticos, mesmo que se trate de um sistema com uma única partícula.

No usual modelo de sistema quântico aberto em que o estado quântico é um elemento do produto dos espaços de Hilbert do sistema de interesse e do ambiente, denotado $S \otimes B$, definimos um Hamiltoniano que descreve uma dinâmica do sistema + ambiente com interação:

$$
\begin{gathered}
H=H_{S}+H_{B}+H_{I}=H_{0}+H_{I} \\
H_{I}=\sum_{n}|n\rangle\langle n| \otimes B_{n} \equiv \sum_{n} A_{n} \otimes B_{n} .
\end{gathered}
$$

Assim, o hamiltoniano de interação, que age não trivialmente em $S \otimes B$, define uma base particular de auto estados em $S$. Essa base de $S$ é inevitavelmente definido pois está associado a $H_{I}$ pelo teorema espectral.

Assume-se que $H_{S}$ comuta com as projeções $A_{n}$ :

$$
\left[H_{0}+H_{I}, A_{n}\right]=\left[H_{0}, A_{n}\right]=0 \text {. }
$$

Portanto, $\left\{A_{n}\right\}$ são observáveis que se conservam pela dinâmica do sistema aberto idealizado.

Passando para a perspectiva de interação, eliminando implicitamente a dinâmica $H_{0}$

$$
H_{I}(t)=e^{i H_{0} t} H_{I} e^{-i H_{0} t}=\sum_{n}|n\rangle\langle n| \otimes B_{n}(t), \quad \text { pois } \quad\left[H_{0}, A_{n}\right]=0
$$

em que $B_{n}(t)=e^{i H_{0} t} B_{n} e^{-i H_{0} t}$.

Seja $U(t)$ o propagador do sistema $S \otimes B$. Para uma derivação dos propagadores dinâmicos gerados pela eq. de Schrödinger, inclusive para o caso geral em que $\left[H\left(t_{1}\right), H\left(t_{2}\right)\right]$ pode ser não nulo para $t_{1} \neq t_{2}$, vide Sakurai (SAKURAI, 1994, pp. 71 e ss.).

Seja $|\Psi(0)\rangle=\sum_{n} C_{n}|n\rangle \otimes|\phi\rangle$ o estado inicial de $S \otimes B$

$$
|\Psi(t)\rangle=U(t)|\Psi(0)\rangle=\sum_{n} C_{n}|n\rangle \otimes\left|\phi_{n}(t)\right\rangle,
$$


pois $\left[H_{I}, A_{n}\right]=0$. Nessa equação $\left|\phi_{n}(t)\right\rangle={ }_{n} U_{B}(t)|\phi\rangle$ e ${ }_{n} U_{B}(t)$ é o propagador no espaço gerado por $B_{n}(t)$.

A equação (4.27) representa o sistema $S \otimes B$ em situação de emaranhamento, em uma superposição que contém os estados $|n\rangle$ do sistema $S$ ainda presentes.

O estado do sistema $S$ é dado por, vide (BREUER; PETRUCCIONE, 2002, p. 215):

$$
\rho_{S}(t)=\operatorname{tr}_{B}\{|\Psi(t)\rangle\langle\Psi(t)|\}=\sum_{n, m} C_{n} C_{m}^{*}|n\rangle\langle m|\left\langle\phi_{m}(t) \mid \phi_{n}(t)\right\rangle
$$

Pela forma da equação (4.28) se constata que os elementos da diagonal principal de $\rho_{S}(t)$ são constantes, pois $\left\langle\phi_{n}(t) \mid \phi_{n}(t)\right\rangle=1$ em virtude da normalização usual dos estados quânticos.

Para analisar os elementos $\left\langle\phi_{n}(t) \mid \phi_{m}(t)\right\rangle$ é preciso considerar que não se trata de estados ortogonais. Para cada dimensão $n$ do sistema $S$ correspondente ao autoestado $|n\rangle$, o estado vinculado em $B,\left|\phi_{n}\right\rangle$ ocupa um espaço multimensional. Em geral, os subespaços de $\left|\phi_{n}\right\rangle$ e $\left|\phi_{m}\right\rangle$ possuem interseção, pois os mesmos graus de liberdade do ambiente podem estar acoplados a diferentes autoestados de $S$. Assim, em geral $\left\langle\phi_{n}(t) \mid \phi_{m}(t)\right\rangle \neq 0$.

Além disso, os experimentos físicos demonstram que a interseção dos subespaços de $\left|\phi_{n}\right\rangle$ e $\left|\phi_{m}\right\rangle$ decresce rapidamente com o tempo. Postula-se, então, a forma a seguir, vide (BREUER; PETRUCCIONE, 2002):

$$
\left|\left\langle\phi_{n}(t) \mid \phi_{m}(t)\right\rangle\right|=\exp \left[\Gamma_{n m}(t)\right], \quad \Gamma_{n m} \leq 0 .
$$

A equação (4.29) acima define uma escala temporal para a decoerência a ser denotada $\tau_{D}$. Para $t>>\tau_{D} \Rightarrow\left\langle\phi_{n}(t) \mid \phi_{m}(t)\right\rangle \rightarrow \delta_{n m}$ e o operador densidade do sistema $S$ toma a forma

$$
\rho_{S}(t) \rightarrow \sum_{n}\left|C_{n}\right|^{2}|n\rangle\langle n|
$$

O sistema $S$ passa, então, a ser constituído por uma mistura incoerente de estados $|n\rangle$, pois são os elementos de fora da diagonal principal que são responsáveis por termos de interferência, da forma $\langle n|A| m\rangle$ para um observável $A$ qualquer. Assim, superposições de estados em $S$ estarão efetivamente destruídos.

Observação 47. Deve-se ter em mente que a coerência de um estado quântico está relacionada com fase relativa entre os autoestados. O estado $|\psi\rangle=\frac{\sqrt{2}}{2}|+\rangle+\frac{\sqrt{2}}{2}|-\rangle$ é uma superposição coerente dos auto estados $|+\rangle$ e $|-\rangle$. Para um estado qualquer em $\mathbb{C}^{2}$, os coeficientes dos autoestados são números complexos e a diferença entre seus argumentos na representação exponencial é a definição de fase relativa.

O estado $|\psi\rangle$ acima, uma superposição coerente com fase relativa nula, é um autoestado de $\hat{\sigma}_{x}$ com autovalor positivo. Por outra, uma superposição de $|+\rangle$ e $|-\rangle$ com fase 
relativa $\pi$ dada por $|\hat{\psi}\rangle=\frac{\sqrt{2}}{2}|+\rangle-\frac{\sqrt{2}}{2}|-\rangle$ representa o outro autoestado de $\hat{\sigma}_{x}$. Para o estado $|\psi\rangle$ que apresenta superposição coerente a representação por operador densidade é dada por

$$
\rho=\left(\begin{array}{ll}
\frac{1}{2} & \frac{1}{2} \\
\frac{1}{2} & \frac{1}{2}
\end{array}\right)
$$

ou seja, os elementos de fora da diagonal principal estão presentes. Por outro, lado uma mistura incoerente de sistemas em igual proporção nos estado $|+\rangle \mathrm{d}|-\rangle$ é representada pelo operador densidade

$$
\rho=\left(\begin{array}{cc}
\frac{1}{2} & 0 \\
0 & \frac{1}{2}
\end{array}\right)
$$

no qual os termos de fora da diagonal principal são nulos. Para uma discussão introdutória sobre coerência, emaranhamento e medição, vide (JACOBS, 1998, pp. 4-7).

Retornando à discussão sobre a decoerência, após essa justificativa da nomenclatura, a evolução dinâmica expressa pela equação (4.30) é chamada decoerência. Emaranhamento e decoerência sâo fenômenos que estão intimamente relacionados, vide eq. (4.27) acima.

Após a realização desse panorama introdutório sobre o fenômenos de dissipação ou amortecimento e decoerência, que são consequência direta de evoluções dinâmicas irreversíveis aplicadas a sistemas quânticos, é interessante destacar algumas de suas semelhanças e diferenças.

A dissipação é um fenômeno relacionado a troca de energia e também ocorre classicamente. A decoerência, por outro lado, não possui paralelo na física clássica. Tal como apresentada acima, a decoerência depende do fenômeno do emaranhamento, que, por sua vez, está relacionado aos postulados da mecânica quântica que definem a estrutura dos sistemas compostos. Classicamente os sitemas são compostos por soma, o que permite que as partes sejam independentes entre si. Quanticamente a composição se dá por multiplicação (produto tensorial).

A independência que é característica dos sistemas clássicos está relacionada à estrutura do espaço e não aos modelos matemáticos que podem ser construídos na teoria. É possível definir classicamente um sistema de equações diferenciais acopladas, no entanto, os subespaçoes definidos pelos vetores de uma base ortogonal são independentes entre si, com relação às operações que podem ser validamente realizadas no espaço.

Observação 48. Na mecânica quântica é possível definir o estado

$$
|\Psi\rangle=\left|\psi_{a}\right\rangle \otimes\left|\psi_{b}\right\rangle \quad \text { em um espaço de Hilbert } \quad \mathcal{H}=\mathcal{H}_{A} \otimes \mathcal{H}_{B}
$$

Se $|\Psi\rangle=\left(\alpha_{1}\left|a_{1}\right\rangle+\alpha_{2}\left|a_{2}\right\rangle\right) \otimes\left(\beta_{1}\left|b_{1}\right\rangle+\beta_{2}\left|b_{2}\right\rangle\right.$ ainda existe independência entre os sistemas, se uma medição for realizada em $\mathcal{H}_{A}$ por meio de um observável qualquer $M$, o resultado 
será um autoestato de $M$, que será denotado por $|\bar{m}\rangle$, e o estado final resultante em $\mathcal{H}_{A} \otimes \mathcal{H}_{B}$ será

$$
|\Psi\rangle_{f}=|\bar{m}\rangle \otimes\left(\beta_{1}\left|b_{1}\right\rangle+\beta_{2}\left|b_{2}\right\rangle\right)
$$

e o vetor de estado no sistema $\mathcal{H}_{B}$ se mantém intacto.

Por outro lado, ao definirmos o estado

$$
|\Psi\rangle=\gamma_{1}\left|a_{1}\right\rangle \otimes\left|b_{1}\right\rangle+\gamma_{2}\left|a_{2}\right\rangle \otimes\left|b_{2}\right\rangle
$$

constataremos que seus elementos constituintes, provenientes de $\mathcal{H}_{A}$ e $\mathcal{H}_{B}$, estão indissociavelmente relacionados.

Ao realizarmos uma medição por um observável compatível com a base $\{|a\rangle\}$, percebemos que

1 - se o resultado da medição for $\left|a_{1}\right\rangle$, o sistema ficará, após a medição, no estado $|\psi\rangle=\left|a_{1}\right\rangle \otimes\left|b_{1}\right\rangle$

2 - se o resultado da medição for $\left|a_{2}\right\rangle$, o sistema ficará, após a medição, no estado $\left|\psi_{2}\right\rangle=\left|a_{2}\right\rangle \otimes\left|b_{2}\right\rangle$.

E, ao realizarmos uma medição em $\mathcal{H}_{B}$ por um observável compatível com a base $\{|b\rangle\}$, no caso 1 obteríamos $\left|b_{1}\right\rangle$ com probabilidade 1 e, no caso 2 , obteríamos $\left|b_{2}\right\rangle$ também com probabilidade 1 .

Em conclusão, a operação de medição realizada em $\mathcal{H}_{A}$ provocou efeitos também em $\mathcal{H}_{B}$. Essa é a natureza do emaranhamento, que decorre das propriedades do produto tensorial, e que não possuem paralelo na física clássica.

Os efeitos irreversíveis provocados pela dissipação e pela decoerência ocorrem em escalas tempo característicos, que são diferentes entre si. Em computação quântica a evolução irreversível de um sistema de 2 qubits é tradicionalmente decomposta em operações chamadas, vide (CHUANG et al., 1998):

1- Amortecimento de amplitude

2- Amortecimento de fase

3- Depolarização

4- troca de bit

5- Troca de fase 
As operações de amortecimento de amplitude e fase estão relacionados a duas escalas de tempo importantes na caracterização de hardware quântico.

A escala de tempo $T_{1}$ está relacionada com uma evolução do tipo

$$
\rho_{0}=\left(\begin{array}{cc}
a & b \\
b^{*} & 1-a
\end{array}\right) \mapsto \rho_{f}(t)=\left(\begin{array}{cc}
\left(a-a_{0}\right) e^{-t / T_{1}}+a_{0} & b \\
b^{*} & \left(a_{0} a\right) e^{-t / T_{1}}+1-a_{0}
\end{array}\right)
$$

e a escala $T_{2}$, com uma evolução

$$
\rho_{0}=\left(\begin{array}{cc}
a & b \\
b^{*} & 1-a
\end{array}\right) \mapsto \rho(t)=\left(\begin{array}{cc}
a & b e^{-t / T_{2}} \\
b^{*} e^{-t / T_{2}} & (1-a)
\end{array}\right)
$$

A operação de amortecimento de amplitude está vinculada aos dois processos (4.31) e (4.32) acima, enquanto a operação de amortecimento de fase está relacionada ao processo (4.32), vide Chuang (CHUANG et al., 1998, sec. 8.3). Pode-se mostrar que $2 T_{2} \leq T_{1}$. Assim, o efeito qualitativo dos processos irreversíveis em um operador densidade é que os termos da diagonal principal mantêm-se mais preservados que os termos de fora da diagonal principal.

Como o amortecimento de amplitude está relacionado à perda de energia e o amortecimento de fase está relacionado à decoerência, vide eq. (4.28) e eq. (4.29), concluímos que a decoerência é um processo dinâmico irreversível mais importante em computação quântica.

As fronteiras da tecnologia no aprimoramento dos dispositivos de hardware para processamento quântico de informação estão relacionadas ao desenvolvimento de arquiteturas que estejam menos susceptíveis a efeitos indesejados do ambiente. Alguns importantes parâmetros vinculados ao hardware são o número de operações lógicas que uma porta quântica pode executar dentro da escala de tempo $T_{2}$ e a taxa de ocorrência de erro nas operações quânticas.

Existem estratégias construtivas para tratar a ocorrência de erros de harware e tornar o processamento quântico de informação tolerante a falhas esta é uma ampla área de pesquisa.

Em artigo de revisão publicado em 2010, Ladd et al. (LADD et al., 2010) resgataram da literatura que taxas de falhas de até $3 \%$ são toleráveis em uma especificação de hardware e podem ser utilizadas robustamente para a construção de um computador quântico, considerando que os recursos de hardware são ilimitados. No entanto, trabalhando com uma estimativa de recursos realista, a taxa de falhas poderia atingir no máximo $10^{-5}$. Vide também Knill (KNILL, 2005), que reporta uma técnica de correção de erro capaz de estruturar uma arquitetura de computação quântica tolerante a falhas com taxas de erros de até $1 \%$. 


\subsection{Teoria da Medição Quântica}

Um dos motivos que dão causa à insatisfação dos físicos com a mecânica quântica é a natureza estocástica associada aos processos de medição. No mais, a dinâmica de um sistema quântico fechado é determinística. É essa contradição que causa incômodo. A teoria dos sistemas quânticos abertos dá um certo alívio, mas ainda sim não resolve, por que, de uma perspectiva cosmológica, ou seja global, se a natureza é quântica, sua evolução deveria ser unitária, portanto, determinística. Uma bela discussão a respeito dos aspectos físicos, filosóficos e existenciais relacionados a esta questão pode ser encontrado em Preskill (PRESKILL, 1997, cap. 3, pp. 48-57).

É interessante começar com um exemplo de medição que é importante para mostrar que a utilização direta e imediata dos axiomas de medição ortogonal apresentados no capítulo não é capaz de levar a um modelo de medição contínua.

Exemplo 49 (Efeito Zeno Quântico). Consideremos a medição de um observável $A$

$$
A=\sum_{n} a_{n}\left|\psi_{n}\right\rangle\left\langle\psi_{n}\right|
$$

em que A tem espectro discreto não degenerado. Uma sequência de medições ortogonais é realizado com período de amostragem $\theta$. A dinâmica do sistema é dada por $i \frac{d}{d t}|\psi(t)\rangle=$ $H|\psi(t)\rangle$. No limite $\theta \rightarrow 0$ o processo de medição será realizado continuamente.

$\left|\psi_{0}\right\rangle=\left|\psi_{n}\right\rangle$ é a condição inicial e para $t$ pequeno temos a aproximação

$$
\begin{aligned}
|\psi(t)\rangle & =\left[I-i H t-\frac{1}{2} H^{2} t^{2}+\ldots\right]\left|\psi_{n}(t)\right\rangle \\
|\psi(\theta)\rangle & =\left|\psi_{n}\right\rangle-\theta H\left|\psi_{n}\right\rangle-\frac{1}{2} H^{2} \theta^{2}\left|\psi_{n}\right\rangle \\
\left\langle\psi_{n} \mid \psi(\theta)\right\rangle & =1-i \theta\langle H\rangle_{n}-\frac{1}{2} \theta^{2}\left\langle H^{2}\right\rangle_{n}
\end{aligned}
$$

onde foram definidos $\langle H\rangle_{n} \equiv\left\langle\psi_{n}|H| \psi_{n}\right\rangle$ e $\left\langle H^{2}\right\rangle_{n}=\left\langle\psi_{n}\left|H^{2}\right| \psi_{n}\right\rangle$

$$
\left|\left\langle\psi_{n} \mid \psi(\theta)\right\rangle\right|^{2}=\left\langle\psi_{n} \mid \psi(\theta)\right\rangle\left\langle\psi_{n} \mid \psi(\theta)\right\rangle^{*}=1-\left[\left\langle H^{2}\right\rangle_{n}-\langle H\rangle_{n}^{2}\right] \theta^{2}+O\left(\theta^{2}\right) .
$$

A probabilidade de se encontrar o sistema no estado $\left|\psi_{n}\right\rangle$ no instante $\theta$ é, então,

$$
p_{n}(\theta)=1-(\Delta E)_{n}^{2} \theta_{2}
$$

em que $\left(\Delta E_{n}^{2}=\left\langle H^{2}\right\rangle_{n}-\langle H\rangle_{n}^{2}\right.$ e a incerteza da energia no estado $\left|\psi_{n}\right\rangle$. Após $\tau=\kappa \theta$, após $\kappa$ amostragens, essa probabilidade é

$$
p_{n}(\tau) \approx\left[1-(\Delta E)_{n}^{2} \theta^{2}\right]^{\kappa}
$$

no limite em que $\tau$ é fixo e $\kappa \rightarrow \infty \Rightarrow \theta=\frac{\tau}{\kappa} \rightarrow 0$

$$
p_{n}(\tau) \approx\left[1-(\Delta E)_{n}^{2} \frac{\tau \theta}{\kappa}\right]^{\kappa} \approx \exp \left[-(\Delta E)_{n}^{2} \tau \theta\right] \rightarrow 1
$$


Assim, em um processo de medição ortogonal contínuo, o estado do sistema se mantém fixo. Esse é o chamado efeito Zeno quântico em analogia ao paradoxo de Zeno. Apesar de ser um efeito idealizado, esta é a ideia intuitiva que nos vem primeiro à cabeça quando pensamos em medição contínua, quando nos baseamos no postulado da medição ortogonal.

A Figura 2 ilustra o efeito zeno quântico em medições contínuas. O parâmetro $\Omega \tau_{0}$ é inversamente proporcional à intensidade da medição realizada. No limite inferior, ocorre o efeito Zeno, pelo qual a intensidade da medição torna o sistema estático.

Figura 2 - Medições fortes e fracas. Figura extraída de Breuer e Petruccione (BREUER; PETRUCCIONE, 2002).

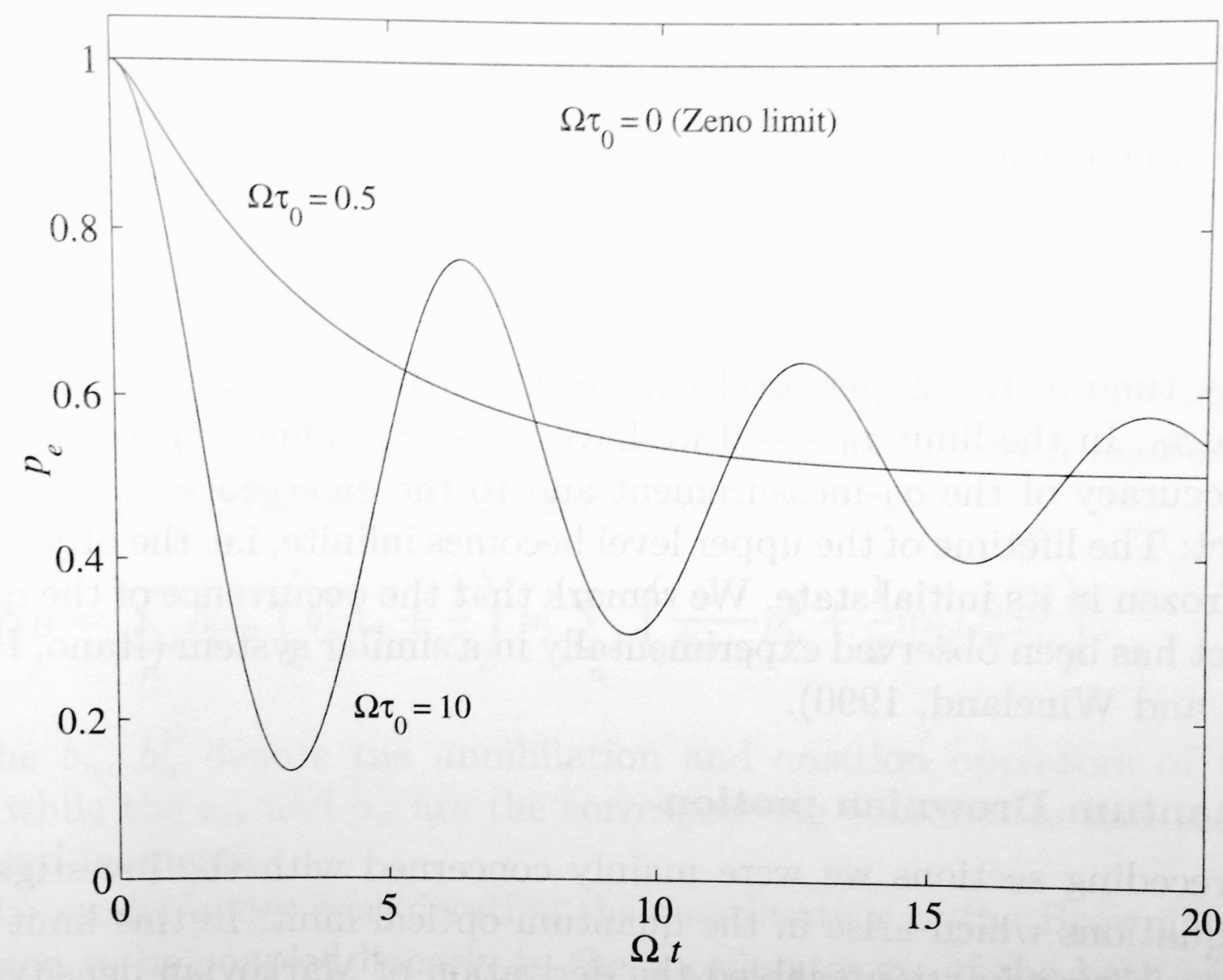

Essa é a motivação para investigarmos processos mais gerais de medição. Em processos de medição generalizados o conjunto de resultados é arbitrário e as medidas podem ser tomadas de forma indireta. Iniciemos, contudo, resgatando os aspectos mais importantes de uma medição ortogonal. 
Definição 50 (Medição Ortogonal). Uma medição ortogonal ou medição de von NeumannLüders em um espaço de Hilbert é baseada em uma família de operadores $\left\{E_{a}\right\}$ tal que

$$
\begin{aligned}
& 1-\quad E_{a}=E_{a}^{\dagger} \\
& 2-E_{a} E_{b}=\delta_{a b} E_{a} \\
& 3-\sum_{a} E_{a}=I
\end{aligned}
$$

As propriedades 1 e 2 definem que os operadores são projeções ortogonais e a propriedade 3 estabelece que a família de operadores é completa. Dado que o sistema se encontra no estado inicial $\rho=|\psi\rangle\langle\psi|$.

Um experimento de medição em um sistema quântico definida em um espaço de Hilbert $\mathcal{H}$ é um conjunto de resultados $\{a\}$ mutuamente exclusivos que, ao ocorrerem, levam o sistema ao estado resultante

$$
\frac{E_{a}|\psi\rangle\langle\psi| E_{a}}{\left\langle\psi\left|E_{a}\right| \psi\right\rangle}
$$

e que podem ocorrer com probabilidade

$$
\operatorname{Prob}(a)=\left\langle\psi\left|E_{a}\right| \psi\right\rangle
$$

Os processos de medição em geral poder seletivos ou não seletivos. Nas medições seletivas uma parcela da população $\rho$ que apresentou ou determinado resultado é separada das demais. Nas medições não seletivas, a população $\rho$ continua a mesma.

Em medições não seletivas, que são mais comuns em aplicações:

$$
|\psi\rangle\left\langle\psi\left|\mapsto \sum_{a} E_{a}\right| \psi\right\rangle\langle\psi| E_{a}
$$

Para uma matriz densidade geral

$$
\rho \mapsto \sum_{a} E_{a} \rho E_{a}
$$

A propriedade 3 é importante para garantir que as probabilidades estejam normalizadas. Assim:

$$
\sum_{a} \operatorname{Prob}(a)=\left\langle\psi\left|\left(\sum E_{a}\right)\right| \psi\right\rangle=1
$$

O experimento definido acima é eficiente, ou seja, sempre alcança um resultado. Nas aplicações é possível também definir e trabalhar com experimentos de medição não eficientes. 
Exemplo 51 (Medição de um observável com espectro contínuo). Em medições ortogonais ideais temos que a matriz densidade resultante de um processo de medição de uma propriedade $B$ cujo subespaço próprio é a imagem do operador de projeção $E(B)$ é dada por

$$
\rho^{\prime}=\frac{E(B) \rho E(B)}{\operatorname{tr}\{E(B) \rho E(B)\}}
$$

A probabilidade do evento $B$ em um experimento de medição é dada por:

$$
P(B)=\operatorname{tr}\{E(B) \rho E(B)\}=\operatorname{tr}\{E(B) \rho\}
$$

considerando a aplicação da propriedade cíclica do traço e o fato de $E(B)$ ser projeção ortogonal. ${ }^{11}$

No caso geral de um operador auto adjunto qualquer, $R$ no espaço de Hilbert, $B$ pode ser um conjunto de Borel de $\mathbb{R}$, correspondente ao evento de que o resultado aleatório do experimento de medição seja um elemento de $B$. O gerador de projeção é definido em termos da família espectral de $R$, vide (BREUER; PETRUCCIONE, 2002, p. 81) para mais detalhes. Seja $\Delta E_{\alpha}$ a família espectral de $R$. Então,

$$
\begin{gathered}
P(B)=\operatorname{tr}\left\{\Delta E_{B} \rho\right\}, \quad \text { e o postulado de medição é dado por } \\
\rho^{\prime}=\frac{\Delta E_{B} \rho \Delta E_{B}}{P(B)} .
\end{gathered}
$$

No caso do operador posição, por exemplo

$$
P(B)=\operatorname{tr}\left\{E_{B} \rho\right\}=\int_{B} d \mu|\psi(x)|^{2} \quad \text { e } \quad \rho^{\prime}=|\phi\rangle\langle\phi|
$$

em que

$$
\phi(x)= \begin{cases}P(B)^{-1} \psi(x), & x \in B \\ 0, & x \notin B .\end{cases}
$$

É importante notar que o resultado acima foi obtido nas condições de uma medição ideal.

Antes de elaborar os processos de medição generalizados, é importante fazer uma defesa do postulado da medição ortogonal, no qual a teoria quântica está baseada. O postulado está vinculado a um sistema quântico fechado $S$ e as medições estão associadas a operadores auto adjuntos quaisquer que agem no mesmo espaço de Hilbert. Nessas

11 A propriedade cíclica do traço, a rigor, não é válida em dimensão infinita. No entanto, na literatura física, é constantemente utilizada nesse contexto. A forma de Lindblad também não é válida em dimensão infinita. 
condições, o postulado apresentado é geral. Caso a medição realizada seja feita em um sistema ampliado $S \otimes \bar{S}$, o processo realizado quando analisado da perspectiva de $S$ isolado poderá ser não ortogonal. É importante também citar o teorema de Neumark, que garante que qualquer processo de medição aplicável em um sistema $S$ é equivalente a uma medição ortogonal em $S \otimes \bar{S}$ para algum espaço de Hilbert $\bar{S}$ que amplia $S$.

Em seguida, apresentaremos um modelo realista de processo de medição que é muito útil para compreender e diferenciar os fenômenos de dissipação, decoerência, medição e emaranhamento, vide (BREUER; PETRUCCIONE, 2002, pp. 261 e ss.). O modelo para a medição é baseado no modelo de decoerência. A interdependência entre medição e decoerência poder ser interpretada a partir de uma afirmação de Breuer e Petruccione "a decoerência, em sua forma mais pura, decorre de certos tipos de interação entre sistema e ambiente que possuem natureza de processos de medição"(BREUER; PETRUCCIONE, 2002, p. 213).

Consideremos o mesmo modelo utilizado para aplicar a decoerência, com $\mathcal{H}=$ $\mathcal{H}_{S} \otimes \mathcal{H}_{n}$ representando a composição dos espaços de estados para o sistema e o medidor. A dinâmica da interação entre sistemas e medidor leva os estados do sistema, representados em uma base $\left|S_{n}\right\rangle$, a ficarem perfeitamente correlacionados com os estados do medidor, representados pelos elementos de um conjunto $\left|M_{n}\right\rangle$. O observável a ser medido é $S=$ $\sum_{n} a_{n}\left|S_{n}\right\rangle\left\langle S_{n}\right|$ para o qual se assume que $\left[S, H_{S M}\right]=0$, em que $H_{S M}$ é o Hamiltoniano de interação entre o sistema e o medidor. Como o observável comuta com o Hamiltioniano, estamos diante de um processo de medição QND, quantum non-demolition. A evolução dinâmica é dada por

$$
\left|\psi_{S M}(0)\right\rangle=\sum_{n} C_{n}\left|S_{n}\right\rangle \otimes|Z M\rangle \mapsto\left|\psi_{S M}(t)\right\rangle=\sum_{n} C_{n}\left|S_{n}\right\rangle \otimes\left|M_{n}\right\rangle .
$$

Como a medição é $Q N D$, os autoestados de $S$ são preservados pela dinâmica. Supondo que os estados de $M$ são também ortogonais $\left\langle M_{n} \mid M_{m}\right\rangle=\delta_{n m}$, a aplicação do postulado da medição ortogonal para $\mathcal{H}_{M}$ com um observável $M=\sum_{n} b_{n}\left|M_{n}\right\rangle\left\langle M_{n}\right|$ no estado $\left|\psi_{S M}(t)\right\rangle$, nos leva a conclusão de que o resultado $b_{n}$ é obtido com probabilidade $\left|C_{n}\right|^{2}$, as mesmas probabilidades iniciais para os estados $\left|S_{n}\right\rangle$ do sistema. E o estado resultante para o sistema será $\left|S_{n}\right\rangle$.

Em um exemplo apresentado por Breuer e Petruccione (BREUER; PETRUCCIONE, 2002, 267) os estados do medidor são tais que $\left\langle M_{m} \mid M_{n}\right\rangle=\exp \left[-K^{2} t^{2}\right]$ para $K>0$, ou seja, a superposição dos subespaços do medidor decaem exponencialmente.

Assim, pode-se concluir que esse modelo representa uma medição ortogonal do observável $S$ no sistema de interesse. No entanto, existe uma ambiguidade na definição da base do medidor, que é resolvida quando se leva em consideração que o medidor em si não é um sistema isolado e, portanto, se encontra em interação com o ambiente. Em decorrência da decoerência que age no medidor, um conjunto ortogonal é selecionado de 
forma preferencial no espaço de Hilbert $\mathcal{H}_{M}$. Esse sistema $\left|M_{n}\right\rangle$ definirá consequentemente uma base em $\mathcal{H}_{S},\left|S_{n}\right\rangle$ que por sua vez estará relacionada ao observável que está sendo medido. A base selecionada pelo ambiente em $\mathcal{H}_{M},\left|M_{n}\right\rangle$, é denominada base da escala de medição, pointer basis. A descrição completa desse modelo pode ser encontrada em (BREUER; PETRUCCIONE, 2002, pp. 261 e ss.). ${ }^{12}$

Apresentaremos a seguir uma estrutura matemática para tratar os experimentos de medição quântica generalizados. A teoria de medição quântica generalizado é baseada na teoria de operações e efeitos operations and effects.

Definição 52 (Medição Quântica Generalizada). Um experimento de medição quântica generalizada realizado em um espaço de resultado $\mathcal{M}$ é definido por famílias de operadores $\left\{F_{m}\right\}_{m \in \mathcal{M}}$ e $\left\{\Omega_{m}\right\}_{m \in \mathcal{M}}$ de tal forma que:

1. A distribuição de probabilidade para os resultados de um experimento de medição é dada por

$$
P(m)=\operatorname{Tr}\left\{F_{m} \rho\right\}
$$

O operador positivo $F_{m}$ é chamado "efeito", e sua família satisfaz

$$
\sum_{\mathcal{M}} F_{m}=I \quad \text { e } \quad \sum_{\mathcal{M}} P(m)=1
$$

2. No caso de medições seletivas, o subconjunto das partículas que compõem o operador densidade para as quais a propriedade $m$ foi dada como verdadeira compõe um operador densidade tal que

$$
\rho_{m}^{\prime}=P(m)^{-1} \Phi_{m}(\rho)
$$

$\Phi_{m}=\Phi_{m}(\rho)$ é um superoperador positivo chamado "operação", que mapeia operadores positivos em operadores positivos. Por consistência

$$
\operatorname{Tr}\{\Phi(\rho)\}=\operatorname{Tr}\left\{F_{m} \rho\right\},
$$

do qual decorre que

$$
\operatorname{Tr}\left\{\rho_{m}^{\prime}\right\}=P(m)^{-1} \operatorname{Tr}\left\{\Phi_{m}(\rho)\right\}=1
$$

3. No caso de medições não seletivos o operador densidade resultante para o sistema é dado por

$$
\rho^{\prime}=\sum_{\mathcal{M}} P(m) \rho_{m}^{\prime}=\sum_{\mathcal{M}} \Phi_{m}(\rho),
$$

que, em função das equações (4.41) e (4.43) é também normalizado

$$
\operatorname{Tr} \rho^{\prime}=\sum_{\mathcal{M}} \operatorname{Tr}\left\{\Phi_{m}(\rho)\right\}=\sum_{\mathcal{M}} \operatorname{Tr}\left\{F_{m} \rho\right\}=\operatorname{Tr}\{\rho\}=1 .
$$

\footnotetext{
12 A análise acima leva a uma discussão sobre a posição fundamental que a medição $Q N D$ ocupa em um modelo de medição real. Não temos conhecimento de outro modelo de medição real, portanto, não sabemos se a hipótese de que $[S, H]=0$ é essencial.
} 
O sistema de medição de von Neumann-Lüders está contido na definição acima se considerarmos uma decomposição da unidade formada por projeções ortogonais $\Delta E_{\alpha} \mathrm{e} \mathrm{um}$ conjunto de intervalos $\Delta r_{\alpha}$ relacionados a essa decomposição. Tomamos $m=\Delta r_{\alpha}$, uma família de operadores positivos dada por $F_{m}=\Delta E_{\alpha}$ e uma família de superoperadores $\Phi_{m}(\rho)=\Delta E_{\alpha} \rho \Delta E_{\alpha}$.

A teoria de operações e efeitos tem um espectro de aplicações que vai além da teoria de medição quântica. Na realidade uma operação pode ser tomada como uma transformação no espaço de estados que descreve evolução dinâmica. A partir dessa técnica é possível deduzir a equação de Lindblad, como um superoperador de evolução, vide Preskill (PRESKILL, 1997, cap. 3 pp. 38 e ss.).

Pelo teorema de representação de Kraus, um superoperador linear e completamente positivo qualquer pode ser representado por uma soma

$$
\Phi(\rho)=\sum_{\kappa} \Omega_{\kappa} \rho \Omega_{\kappa}^{\dagger}
$$

para um conjunto de operadores $\Omega_{m}$ que satisfaça a condição de normalização

$$
\sum_{\kappa} \Omega_{\kappa}^{\dagger} \Omega_{\kappa}=I
$$

Temos, então, que, para um sistema de medição como definido acima, a forma mais geral para as operações é dada por

$$
\begin{gathered}
\Phi_{m}(\rho)=\sum_{\kappa} \Omega_{m \kappa} \rho \Omega_{m \kappa}^{\dagger}, \quad \text { e para os efeitos } \\
F_{m}=\sum_{\kappa} \Omega_{m \kappa}^{\dagger} \Omega_{m \kappa} .
\end{gathered}
$$

Figura 3 - Diagrama esquemático de um experimento de medição generalizada. Figura extraída de Breuer e Petruccione (BREUER; PETRUCCIONE, 2002).

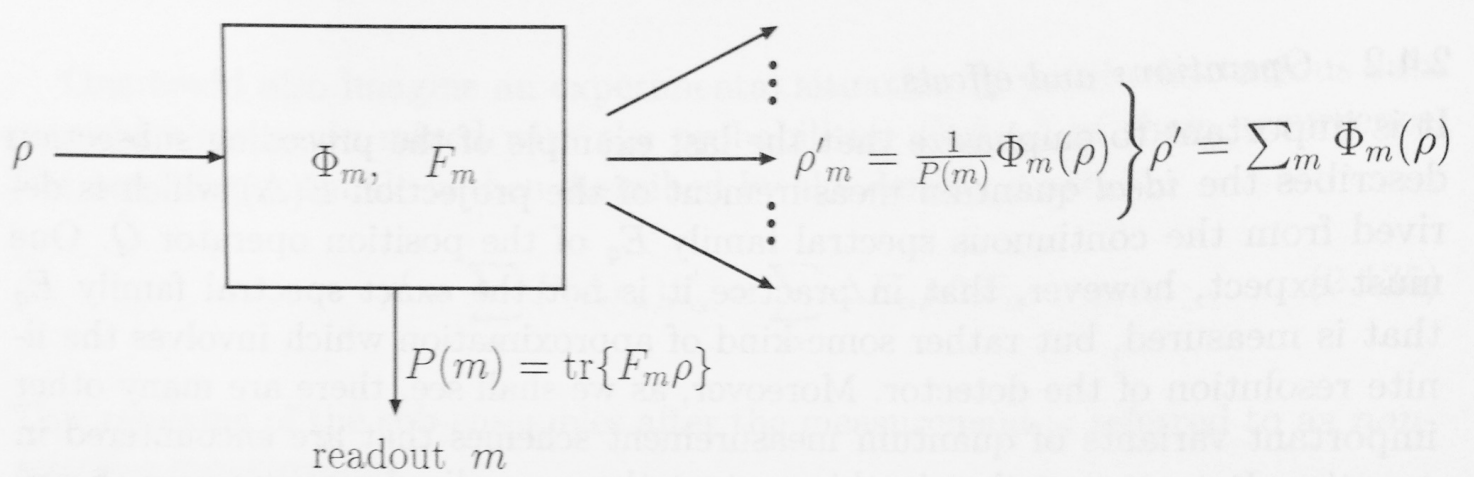


A Figura 3 apresenta um diagrama representando um sistema de medição generalizada, descrevendo a medição como um processo, que pode ser ou não seletivo, a partir do qual se obtém um resultado, descrito por uma distribuição de probabilidade.

O sistema de medição generalizado definido acima é frequentemente denominado POVM de positive operator valued measure. Essa denominação decorre do fato de ser possível definir uma medida em $\mathcal{M}$ que toma valores em um conjunto de operadores positivos, vide (PRESKILL, 1997; REED; SIMON, 1980).

Exemplo 53 (Medição na Esfera de Bloch). Sejam os operadores

$$
\begin{aligned}
& F_{0}=\left(\frac{1+\varepsilon}{2}\right)|0\rangle\left\langle 0\left|+\left(\frac{1-\varepsilon}{2}\right)\right| 1\right\rangle\langle 1| \\
& F_{1}=\left(\frac{1-\varepsilon}{2}\right)|0\rangle\left\langle 0\left|+\left(\frac{1+\varepsilon}{2}\right)\right| 1\right\rangle\langle 1| \\
& \Omega_{0}=\sqrt{\left(\frac{1+\varepsilon}{2}\right)}|0\rangle\left\langle 0\left|+\sqrt{\frac{1-\varepsilon}{2}}\right| 1\right\rangle\langle 1| \\
& \Omega_{1}=\sqrt{\frac{1-\varepsilon}{2}}|0\rangle\left\langle 0\left|+\sqrt{\frac{1+\varepsilon}{2}}\right| 1\right\rangle\langle 1| .
\end{aligned}
$$

Esses operadores definem um sistema de medição generalizado ou POVM para o qual o estado resultante do sistema após a medição é dado por:

$$
\left|\psi_{j}\right\rangle=\frac{\Omega_{j}|\psi\rangle}{\sqrt{p_{j}}} \quad j \in\{0,1\}
$$

Temos, então, que aproximadamente:

$$
\begin{aligned}
& \left|\psi_{0}\right\rangle \approx \alpha\left(1+\varepsilon|\beta|^{2}\right)|0\rangle+\beta\left(1-\varepsilon|\alpha|^{2}\right)|1\rangle \\
& \left|\psi_{1}\right\rangle \approx \alpha\left(1-\varepsilon|\beta|^{2}\right)|0\rangle+\beta\left(1+\varepsilon|\alpha|^{2}\right)|1\rangle,
\end{aligned}
$$

para um estado inicial $|\psi\rangle=\alpha|0\rangle+\beta|1\rangle$.

Para $\varepsilon<<1$, o resultado da medição, 0 ou 1, é bastante aleatório tendo em vista que $p_{0} \approx p_{1} \approx \frac{1}{2}$. Uma sequência de resultados, no entanto, pode ser estatisticamente analisada para acumular, a cada novo resultado, mais informação a respeito do estado $|\psi\rangle$.

Esse experimento de medição, por um lado, mantém o estado inicial praticamente intacto, em contrapartida, a quantidade de informação que adquire a cada experimento de medição é praticamente nula. À medida que uma sequência de medições é realizada o estado do sistema, é arrastado em direção a $|0\rangle$ ou $|1\rangle$ com probabilidade inicial dada por $|\alpha|^{2}$ e $|\beta|^{2}$ respectivamente. 
Medições desse tipo são classificadas como medições fracas em contraposição às chamadas medições fortes, para às quais a taxa de aquisição de informação é alta assim como a reação do estado ao processo de medição.

Muitos exemplos de experimentos de medição $P O V M$ em sistemas quânticos de 2 níves, qubits, podem ser encontrado em Brun (BRUN, 2001). Nesse artigo, o autor demonstra com modelos simples a interação de um sistema com seu meio ambiente e seus efeitos principais. Outro assunto tratado são as trajetórias quânticas, apresentado de forma didática e esclarecedora dos fenômenos físicos envolvidos na evolução de sistemas quânticos abertos.

Até esse momento, no tratamento dos sistemas quânticos abertos, os modelos utilizados foram determinísticos, baseados em equações mestras markovianas. O tratamento de dinâmica por meio de equações mestras permite que sistemas estocásticos sejam modelados por uma equação dinâmica capaz de captar o comportamento médio de uma população ou o comportamento médio de uma partícula em uma série de experimentos idênticos repetidos por muitas vezes. As equações mestras adequam-se ao tratamento de sistemas que apresentam saltos e de sistemas que apresentam processos de difusão aleatórias. E também ao tratamento de sistemas determinísticos que apresentam estado modelado por distribuição de probabilidade, vide (GARDINER, 2003).

O tratamento direto dos fenômenos estocásticos em sistemas dinâmicos leva ao desenvolvimento de modelos que se baseiam em processos estocásticos. Na dinâmica de sistemas quânticos abertos, os processos estocásticos podem ser incorporados:

1. à equação mestra, portanto, à dinâmica do estado do sistema modelado por operador densidade na perspectiva de Schrödinger;

2. diretamente ao estado puro de um sistema, portanto, também à dinâmica do sistema na perspectiva de Schröedinger;

3. à equação dinâmica dos observáveis, ou seja, na perspectiva de Heisenberg.

Os modelos acima levam respectivamente à equação mestra estocástica, SME, à equação de Schrödinger estocástica, SSE, e à equação de Langevin quântica ou equação diferencial estocástica quântica, QSDE.

Nos três casos acima, quando os modelos matemáticos são usados para descrever a dinâmica do mesmo sistema físico os resultados são os mesmos, na média das variáveis dinâmicas, mas podem ser completamente diferentes em trajetórias amostrais.

Tomando, por exemplo, o caso das equações mestras é possível, para uma mesma equação de Lindblad, modelar comportamentos que têm naturezas completamente dife- 
rentes. Ao incorporar um processo estocástico de Poisson, é possível modelar sistemas que apresentem saltos, por exemplo, dinâmicas associadas a experimentos de medição fortes. Incorporando à mesma equação um processo de Wiener é possível modelar sistemas que apresentem difusão, por exemplo, no caso de medições fracas contínuas. É importante frisar que a equação mestra pode ser exatamente a mesma, vide (WISEMAN; MILBURN, 2010, p. 211).

Uma vez definido o processo estocástico a ser incorporado à equação mestra, para cada realização do ruído estará associada uma trajetória do sistema. Há uma bela discussão física associada à condição real ou fictícia dessas trajetórias, vide, por exemplo, Wiseman (WISEMAN, 2003) e as referências citadas.

Um importante aspecto na definição dos modelos é a escolha do processo estocástico que será utilizado para representar os aspectos não determinísticos do sistema. A cada escolha realizada corresponde um diferente unravelling.

Retomando a discussão a respeito da medição contínua, lembramos que anteriormente foi apresentado um modelo para o efeito Zeno quântico. Na nomenclatura definida, trata-se de um modelo de medição contínuo forte. Apresentaremos a seguir um modelo de medição contínua para o qual será definido um parâmetro para definir se a medição é mais fraca ou mais forte.

Seja $S$ o sistema de interesse no qual desejamos realizar um experimento de medição definido pelo observável $A$ e seja $P$ um sistema sonda. Para dar origem a um processo de medição indireta realiza-se o acoplamento dinâmico entre a sonda e o sistema de interesse. O observável $A$ é acoplado ao operador posição da sonda, $Q$, por meio de um Hamiltoniano de interação $H_{I}(t)=g(t) A Q$, onde $g(t)$ é a constante de acoplamento.

Figura 4 - Representação esquemática de um experimento de medição indireta. Figura extraída de Breuer e Petruccione (BREUER; PETRUCCIONE, 2002).

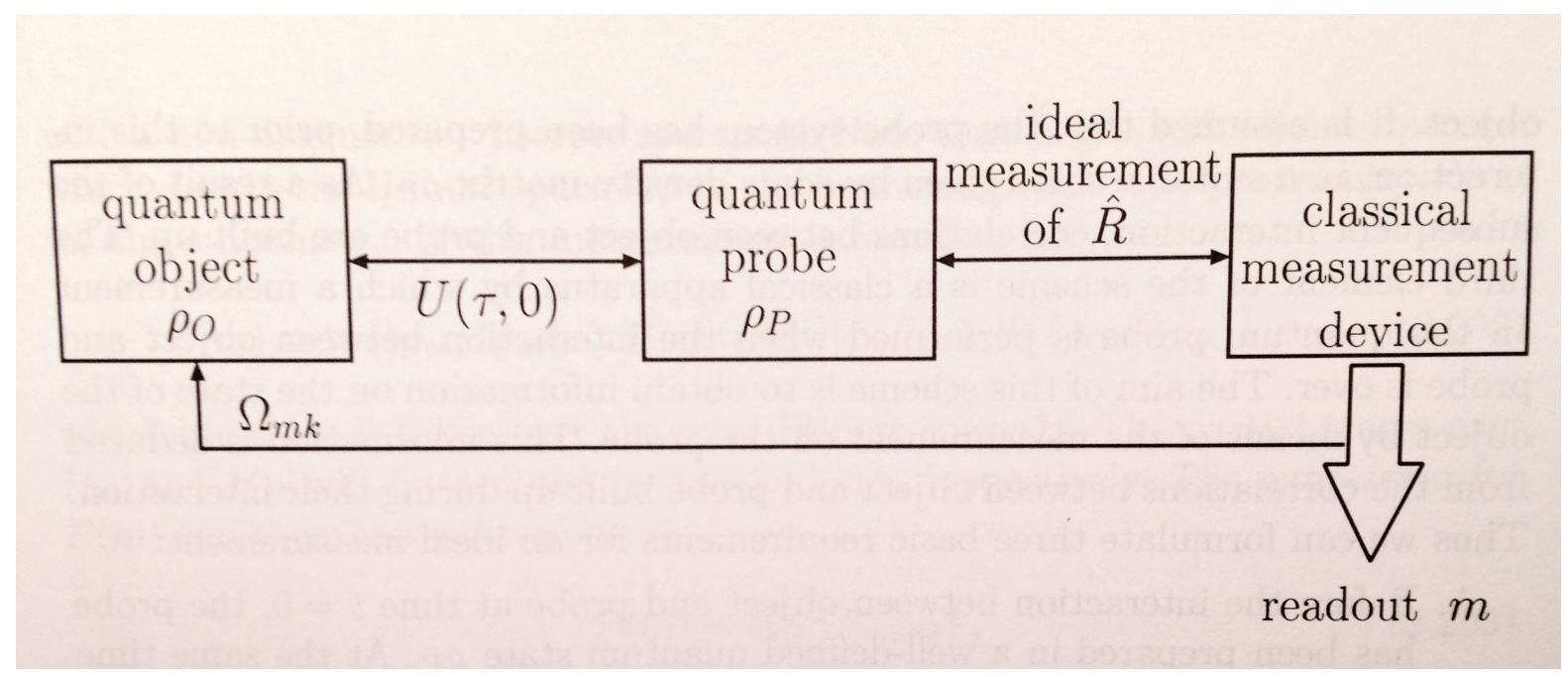

A Figura 4 ilustra um aparato de medição indireta. O sistema de interesse interage 
com uma sonda. O experimento de medição é realizada na sonda, obtendo-se informação a respeito do sistema de interesse.

Por meio de um POVM definindo por

$$
\Omega_{p}=\exp (-i H \Delta t)\langle p|\exp (-i A Q \Delta t)| \phi\rangle
$$

em que $\Delta t$ é um intervalo de tempo a ser tomado como infinitesimal, $|p\rangle$ são os autoestados do operador momento linear da sonda, $P$, conjugado a $Q$ e $|\phi\rangle$ o estado da sonda. A medição, em um intervalo de tempo $\Delta t$ é dada por

$$
\rho(t+\Delta t)=\int d p \Omega_{p} \rho(t) \Omega_{p}^{\dagger}
$$

No limite em que $\Delta t \rightarrow 0$, a equação dinâmica do processo de medição é dada por, vide (BREUER; PETRUCCIONE, 2002, pp. 162 e ss.):

$$
\frac{d}{d t} \rho(t)=-i[H, \rho]+\sigma_{Q}^{2} \mathcal{D}[A] \rho
$$

A partir de uma manipulação da relação de incerteza para as variáveis $P$ e $Q$ no sistema sonda, extrai-se que:

$$
\sigma_{A}^{2} \sigma_{Q}^{2} \geq \frac{1}{4}
$$

em que $\sigma_{A}^{2}$ e $\sigma_{Q}^{2}$ são quantidades relacionadas a $\left\langle A^{2}\right\rangle$ e $\left\langle Q^{2}\right\rangle$. A relação entre $\left\langle A^{2}\right\rangle$ e $\left\langle P^{2}\right\rangle$ fica mais clara ao analisarmos uma relação geral deduzida para medições indiretas, vide (BREUER; PETRUCCIONE, 2002, pp. 93-99), onde se estabelece:

$$
\operatorname{Var}(a)=\operatorname{Var}(A)+\frac{1}{G^{2}} \operatorname{Var}(P),
$$

em que $G$ é proporcional ao acoplamento médio entre os observáveis do sistema de interesse e da sonda ao longo do intervalo de medição e $\operatorname{Var}(a)$ é a variância da medida de $A$ realizada indiretamente pela sonda. Pela análise das equações (4.57) e (4.59) vemos que a acuracidade da medição e a reação dinâmica do sistema ao processo de medição são proporcionais às incertezas na sonda.

A equação (4.57) seria mais propriamente escrita como

$$
\dot{\rho}=-i[H, \rho]+G^{2} \sigma_{Q}^{2} \mathcal{D}[A] \rho,
$$

o que a deixaria compatível com a equação de medição contínua geral a ser apresentada. ${ }^{13}$

13 De fato, a dedução da equação (4.57) se baseia em duas aproximações que não são fisicamente naturais. Ao realizar os cálculos deixados implícitos na exposição dos autores, fomos levados à suposição que as aproximações realizadas pelos autores poderiam ser eliminadas a partir do uso do cálculo estocástico, uma vez que uma das aproximações, $\sigma_{Q}^{2}=\lim _{t \rightarrow 0} \Delta t\left\langle Q^{2}\right\rangle$, é utilizada para transformar um infinitesinal quadrático em $d t$ em um diferencial linear. Esse tipo de transformação é típico do cálculo estocástico. No entanto, escolhemos apresentar esse exemplo por se tratar de um exemplo não trivial da aplicação de medições indiretas. Lembramos também que a estrutura da equação, que apresenta a forma de Lindblad é qualitativamente correta. De todo modo, a relação (4.59), válida para medições indiretas, é importante por si só. 
No formato acima, as equações deixam evidente o efeito das medições mais fracas ou mais fortes na acuracidade e no estado. No limite em que $\operatorname{Var}(P) \rightarrow 0$ na equação (4.59), o processo de medição tende a uma medição ideal do observável $A$ no sistema, com o consequente calapso do estado do sistema e um autoestado de $A$. A análise conjunta das equações (4.59) e (4.60) também deixa evidente o papel desempenhado pelo parâmetro de acoplamento $G$, ao estabelecer o balanço entre a acuracidade da medição do observável $A$ e a intensidade dos efeitos indesejados provocados pelo processo de medição.

Apresentamos a seguir um exemplo de medição POVM que será utilizado como motivação para a apresentação das equações de medição quântica contínuas.

Exemplo 54 (Medição Gaussiana). Seja um experimento de medição generalizado definido por

$$
\Omega_{m}=\frac{1}{\mathcal{N}} \sum_{n} e^{-\kappa(n-m)^{2} / 4}|n\rangle\langle n| .
$$

O sistema em consideração possui $n$ dimensões e seu observável $N$ possui um conjunto de autovalores dado por $\{1,2, \ldots, n\} . \mathcal{N}$ é uma constante de normalização que garante que

$$
\sum_{m} F_{m}=\Omega_{m}^{\dagger} \Omega_{m}=I
$$

Após a realização de uma medição seletiva e obter o resultado $m$, tomando por estado inicial um operador densidade totalmente misturado (mixed), ou seja $\rho \alpha I$, temos como estado resultante

$$
\rho_{f}=\frac{\Omega_{m} \rho \Omega^{\dagger}}{\operatorname{tr}\left\{\Omega_{m} \rho \Omega^{\dagger}\right\}}=\frac{1}{\mathcal{N}} \sum_{n} e^{-\kappa(n-m)^{2} / 2}|n\rangle\langle n| .
$$

Assim, após a medição, o estado final apresenta um pico centrado no autovalor $m$, resultado do experimento de medição. A largura do pico é dada por $1 / \sqrt{\kappa}$. Quanto maior o valor de $\kappa$ maior o estado de certeza, ou estado de conhecimento, a respeito do estado do sistema como consequência do processo de medição. As medições caracterizadas por valores de $\kappa$ grandes são medições fortes, ou inversamente medições fracas.

Tornando o exemplo concreto, consideremos que o sistema sob análise possui uma dinâmica relacionada ao momento angular e que o observável em questão seja o momento angular na direção $Z, J_{z}$. Utilizando os resultados da teoria de medição contínua, pode-se mostrar que o resultado da medição será dado por

$$
P(Y, t)=\frac{1}{2 j+1} \sum_{n=-j}^{j} \sqrt{\frac{4 \kappa t}{\pi}} e^{-4 \kappa t(Y-n)^{2}},
$$

em que $P(Y, t)$ é a distribuição de probabilidade da variável aleatória $Y$ que representa o resultado da medição realizada

$$
Y(t)=\frac{1}{t} \int_{0}^{t}\left\langle J_{z}(t)\right\rangle d t+\frac{1}{t \sqrt{8 \kappa}} \int_{0}^{t} d W,
$$


e $d W$ é o incremento de um processo de Wiener.

Pode-se perceber que, à medida que o tempo de medição cresce, aumenta a probabilidade de a variável $Y$ se encontrar próxima aos $2 j+1$ valores-próprios do observável $J_{z}$.

O parâmetro de medição $\kappa$ define, em conjunto com o tempo $t$, a velocidade com que o processo de medição irá convergir para um dos valores próprios do observável sob medição. Interessante notar que na solução das equações dinâmicas para a obtenção da distribuição de probabilidade de $Y$, Jacobs e Steck (JACOBS; STECK, 2006) também utilizaram a hipótese de que o observável e o Hamiltoniano do sistema comutavam entre si. Apontaram, no entanto, trabalhos que discutem soluções mais gerais.

Apresentamos a seguir uma forma das equações dinâmicas estocásticas que modelam processos de medição contínuas, o conjunto de equações utilizado por Jacobs e Steck para resolver o problema da medição contínua no caso do observável $J_{z}$, como apresentando acima. As equações dinâmicas abaixo incorporam ruído gaussiano. Há outros conjuntos de equações dinâmicos incorporando outros tipos de ruído, em especial ruído de Poisson, quando os sistemas físicos estão associados por exemplo a contagem de fótons. Trata-se, portanto, de diferentes unravellings, vide (GARDINER; ZOLLER, 2004; WISEMAN; MILBURN, 2010; JACOBS, 2014; JACOBS; STECK, 2006).

Em uma medição contínua extrai-se informação ininterruptamente do sistema. Conforme foi apresentado nos exemplos discutidos acima, busca-se uma solução de compromisso entre a taxa de extração de informação e a taxa de perturbação do estado do sistema. Em uma sequência de medições realizadas em um sistema quântico, para que a perturbação no estado possa ser mantido dentro de limites aceitáveis, a quantidade de informação extraída em cada amostra será diminuída à medida em que a frequência de amostragem estiver sendo aumentada. Para isso, o parâmetro de medição $\kappa$ deverá ser feito proporcional ao intervalo entre as medições. O intervalo de tempo também pode ser visto como a duração de cada medição.

Os operadores $\Omega_{\alpha}$ que definem o experimento de medição generalizada que será utilizado no procedimento de amostragem são descritos por

$$
\begin{aligned}
& \Omega_{\alpha}=\left(\frac{4 \kappa}{\pi d t}\right)^{\frac{1}{4}} \int_{-\infty}^{+\infty} \exp \left\{-2 \kappa \frac{(\alpha-x d t)^{2}}{d t}\right\}|x\rangle\langle x| d x \\
& \Omega_{\alpha}=\left(\frac{4 \kappa}{\pi d t}\right)^{\frac{1}{4}} \exp \left\{-\frac{2 \kappa}{d t}(\alpha-X d t)^{2}\right\}
\end{aligned}
$$

em que $X$ é o observável que está sendo medido e $|x\rangle$ seu conjunto de autoestados.

O experimento $P O V M$ foi definido de tal forma que a distribuição de probabilidade 
do parâmetro $\alpha$ seja equivalente a uma variável aleatória dada por

$$
\alpha=\langle X\rangle d t+\frac{d W}{\sqrt{8 \kappa}}
$$

em que $d W$ é o incremento de um processo de Wiener.

Ao aplicar os operadores de medição a um sistema inicialment no estado $|\psi\rangle$, é possível obter a seguinte equação para a evolução do estado $|\psi(t)\rangle$, vide (JACOBS, 2014, cap. 3):

$$
d|\psi(t)\rangle=\left\{-\kappa(X-\langle X\rangle)^{2} d t+\sqrt{2 \kappa}(X-\langle X\rangle) d W\right\}|\psi(t)\rangle
$$

que é uma equação de Schrödinger estocástica.

Note-se que a equação acima é não linear, ou seja, o equivalente clássico do operador de evolução da equação acima é em geral não linear. Fisicamente, essa questão é muito importante, pois ao se introduzir a dinâmica relacionada a medição de um observável $X$, surgem na evolução termos associados ao operador $X^{2}$.

Observação 55. Esse fato é muito importante na análise das questões relacionadas à contralabilidade de sistemas em dimensão infinita, por que, conforme visto no capítulo anterior, uma das condições necessárias para que um sistema em dimensão infinita seja controlável é que a sua algebra dinâmica tenha dimensão infinita. E de acordo com os resultados analisados no capítulo anterior, parece que a presença de um operador dinâmico não linear é uma condição essencial para que a álgebra dinâmica do sistema tenha dimensão infinita.

Dessa forma, dado um sistema de controle em malha aberta,

$$
d|\psi\rangle=\left(H_{0}+\sum_{i=1}^{n} u_{i} H_{i}\right)|\psi\rangle,
$$

ao analisar suas condições de controlabilidade em malha fechada, devemos incorporar à sua álgebra de controle os operadores auto adjuntos que representam os observáveis que serão medidos, de acordo com o regime em que o sistema de controle será monitorado. Assim, no caso de medição contínua de um observável $A \in\left\{H_{0} \cdots H_{n}\right\}$, de acordo com a equação dinâmica apresentada acima, tanto $A$ quanto $A^{2}$ devem ser incorporados à algebra de controle, que é o parâmetro utilizado para se determinar se um sistema é controlável ou não. ${ }^{14}$

Contudo, é necessário ter cautela com as conclusões acima tendo em vista que a análise realizada é apenas qualitativa. É preciso antes investigar a intensidade dos efeitos dos observáveis na dinâmica de um sistema de controle de natureza quântica.

14 Mais propriamente, dever-se-ia dizer que uma determinada função dos operadores $A$ e $A^{2}$ é que será incorporada a um conjunto particular que, se se demonstrar ter a estrutura de uma álgebra, poderíamos denominar de álgebra de medição. 
Uma questão bastante interesse na teoria de controle está relacionada à dinâmica livre do sistema, que pode restringir bastante suas condições de controle. De acordo com a análise acima, em sistemas de natureza quântica esse problema poderia ser amenizado ou até mesmo resolvido, caso seja incluído um processo de medição do observável $H_{0}$, o Hamiltoniano livre do sistema.

Outra questão que talvez seja de interesse é a análise do efeito dinâmico da operação de medição tendo em vista que os observáveis podem comutar ou não com os Hamiltonianos do sistema de controle. Esse tipo de análise define se as operações de medição são do tipo $Q N D$ com relação à álgebra de controle, ou com relação à alguma subalgebra da álgebra de controle. O surgimento de operadores geradores de evolução dinâmica formados pelo comutador do observável com alguns dos elementos da álgebra de controle pode ter como consequência o enriquecimento desta. E pode ser um dos fatores determinantes para se estabelecer quantitativamente a controlabilidade, como observado acima.

Sobre a perturbação causada pela medição no estado do sistema, é importante analisar o subespaço do conjunto alcançável que é gerado exclusivamente por operadores da álgebra de medição ${ }^{15}$ na geração de ruído no estado do sistema. É possível que o enriquecimento da álgebra de controle esteja associado a um custo de adição de ruído. Assim, alguns subespaços do conjunto de estados alcançáveis podem estar vinculados a uma maior quantidade de ruído e sujeitos, portanto, a uma taxa de erros maior.

Consideremos também um sistema de controle QND associado a um conjunto completo de operadores auto adjuntos tal que seus Hamiltonianos e observáveis sejam elementos do conjunto. Tal sistema dá origem a um sistema controlável na variedade integral associado ao conjunto completo. Pelas propriedades da medição QND, o ruído de medição, que tem fundamento no princípio de Heisenberg, gera perturbações em variáveis canônicas que estão associadas a operadores que não pertencem ao conjunto completo definido. Assim, em teoria, o ruído observável pela realização de experimentos de medição com operadores auto adjuntos do conjunto completo pode ser tão baixo quanto se queira. Interessante acrescentar nesse ponto que a teoria de probabilidade quântica define que álgebras de operadores que comutam estão associadas a espaços de probabilidade clássicos, portanto, existe alguma consistência entre as análises realizadas e o cálculo estocástico quântico. A adição de uma medição não compatível poderia ter como efeito retirar o sistema da variedade integral tornando-o não controlável, ou adicionar ruído ao estado do sistema.

Isso nos leva a mais uma conjectura interessante, a de que subespaços controláveis maximais com ruído dinâmico tão baixo quanto se queira em sistemas dinâmicos quânticos

15 Os operadores gerados por comutação entre a álgebra de observação e a álgebra de controle também deve ser analisados. 
de dimensão infinita estejam associados a conjuntos completos de observáveis em uma relação biunívoca. É certo que álgebra dinâmica de um sistema possui uma partição em álgebras de operadores que comutam entre si.

Retornando às equações dinâmicas da medição contínua, a partir da $S S E$ definida acima, é possível obter a equação mestra estocástica associada

$$
d \rho=2 \kappa \mathcal{D}[X] \rho d t+\sqrt{2 \kappa}(x \rho+\rho X-2\langle X\rangle \rho) d W
$$

em que usamos a relação $2 \mathcal{D}[X] \rho=-[X,[X, \rho]]$ para um operador auto adjunto $X=X^{\dagger}$.

Jacobs (JACOBS, 2014) afirma que a evolução devida a um Hamiltoniano $H$ pode ser livremente adicionada utilizando um argumento baseado na manipulação dos diferenciais $d t$ e $d W$.

Assim a equação mestra estocástica geral para medição contínua de um observável e adição de ruído gaussiano é dada por:

$$
d \rho=-i[H, \rho] d t+2 \kappa \mathcal{D}[X] \rho d t+\sqrt{2 \kappa}(X \rho+\rho X-2\langle X\rangle \rho) d W
$$

A constante $\kappa$ é chamada intensidade de medição e define, ao mesmo tempo, a taxa de extração de informação e a taxa de perturbação do estado em consequência da dinâmica de medição. O sinal de medição associado à equação (4.70), conforme a definição do POVM acima, é dado por

$$
d y=\langle X\rangle d t+\frac{1}{\sqrt{8 \kappa}} d W
$$

O terceiro termo da equação (4.70) é frequentemente obreviado por

$$
\mathcal{H}[\Lambda] \rho=\Lambda \rho+\rho \Lambda^{\dagger}-\left\langle\left(\Lambda+\Lambda^{\dagger}\right)\right\rangle \rho
$$

Os processos de medição também podem ser realizado em paralelo, ou seja, simultaneamente, no mesmo sistema, valendo a equação abaixo

$$
d \rho=-i[H, \rho] d t+\sum_{i} 2 \kappa_{i} \mathcal{D}\left[X_{i}\right] \rho d t+\sum_{i} \sqrt{2 \kappa_{i}} \mathcal{H}\left[X_{i}\right] \rho d W
$$

Observação 56. A semelhança entre as equações (4.70), (4.59) e (4.57) é interessante. Primeiro devemos notar que $\langle d W\rangle=0$, portanto, a equação mestra determinística associada à equação (4.70) é a equação de Lindblad, similar às equações (4.59) e (4.57). A equação (4.59), como já apontamos, é mais viável, pois possui explicitamente um termo proporcional à intensidade de medição, no caso $G^{2}$. De acordo com a técnica de dedução da equação analisada em (BREUER; PETRUCCIONE, 2002) o parâmetro de intensidade de medição $G^{2}$ pode ser facilmente incorporado ao modelo. 
No entanto, o processo de dedução utilizado pelos autores para chegar à eq. (4.57), parece conter duas hipóteses demasiadamente forçadas. Ambas foram utilizadas pelos autores para se verem livres de infinitesimais indesejados. Considerando que os autores, em suas próprias palavras, estavam tratando de dinâmica coerente e não coerente, o problema com os infinitesimais pode indicar que um modelo utilizando $d t$ e $d W$ poderia eliminar as hipóteses não naturais utilizadas. Também poderia ser buscada uma dedução baseada na teoria de probabilidade quântica que ruídos na forma de operadores com relação de composição que generaliza a regra de Itô.

A equação (4.57) parece ser mais geral que a equação (4.70), pois seu método de dedução não faz hipóteses a respeito da natureza do ruído de medição. Assim, seria interessante obter uma equação de medição contínua, deduzida a partir de princípios físicos, não vinculada a um tipo específico de ruído.

Apresentamos a seguir uma relação entre as equações de medição contínua e o filtro de Kalman-Bucy, conforme encontramos na literatura física na área de medição de sistemas quânticos, vide (WISEMAN; MILBURN, 2010; JACOBS, 2014).

Exemplo 57. A equação mestra estocástica de um sistema quântico linear, ou seja, com Hamiltoniano quadrático em um conjunto de pares de variáveis canônicos, quando utilizada para apresentar estimativas para as médias das variáveis de estado, possui uma forma similar à do filtro de Kalman-Bucy.

No caso de um oscilador harmônico quântico em uma dimensão associado a um processo de medição contínua de sua posição, a equação de evolução para as médias das variáveis canônicas é dada por:

$$
\begin{aligned}
& d\langle x\rangle=\langle p\rangle d t+\sqrt{8 \kappa} V_{x} d W \\
& d\langle p\rangle=-\langle x\rangle d t+\sqrt{8 \kappa} C_{x p} d W
\end{aligned}
$$

e para os elementos da matriz de covariância

$$
\begin{aligned}
\dot{V}_{x} & =2 C_{x p}-8 \kappa V_{x}^{2} \\
\dot{V}_{p} & =2 \kappa-8 \kappa C_{x p}^{2}-2 C_{x p} \\
\dot{C}_{x p} & =V_{p}-8 \kappa V_{x} C_{x p}-V_{x}
\end{aligned}
$$

As equações (4.74) e (4.75) acima foram, portanto, derivadas para estimar as propriedades de um sistema quântico linear com estados gaussianos para o qual a posição é continuamente monitorada.

As equações acima são também as equações dinâmicas para o sistema utilizado para estimar o estado de um oscilador clássico também sujeito a ruído de flutuação e para 
o qual a posição é também monitorada com precisão finita e proporcional a $\kappa$ :

$$
\begin{aligned}
& d x=p d t \\
& d p=-x d t+\sqrt{2 \kappa} d W_{c} .
\end{aligned}
$$

Classicamente, no entanto, os ruídos de medição e de flutuação não estão correlacionadas.

Utilizando a relação de inovação $d W=\sqrt{8 \kappa}(d y-\langle x\rangle d t)$ a dinâmica do estimador definido pela equação (4.74) fica:

$$
d\left(\begin{array}{c}
\langle x\rangle \\
\langle p\rangle
\end{array}\right)=\left(\begin{array}{cc}
-8 \kappa & 1 \\
-1 & 0
\end{array}\right)\left(\begin{array}{c}
\langle x\rangle \\
\langle p\rangle
\end{array}\right) d t-8 \kappa\left(\begin{array}{c}
V_{x}^{s s} \\
C_{x p}^{s s}
\end{array}\right) d y
$$

em que $d y$ é o valor medido e $V_{x}^{s s}$ e $C_{x p}^{s s}$ são os valores de regime para os quais a equação (4.75) converge, vide (JACOBS, 2014, pp. 104 e ss.) para maiores detalhes.

A analogia entre sistemas clássicos e sistemas quânticos na aplicação da teoria de controle com realimentação é bastante presente na literatura. Classicamente, o estado de conhecimento que um observador possui a respeito de seu estado dinâmico é expresso por uma distribuição de probabilidade no espaço de estados do sistema. Quanticamente, o estado de conhecimento é representado pelo operador densidade. Por meio de processos de medição, o observador coleta informação a respeito do estado do sistema e a utiliza para atualizar seu estado-de-conhecimento, state of Knowledge. O observador constrói assim um modelo para estimar o estado de um sistema dinâmico. Para sistemas clássicos não lineares, em geral, esse modelo é composto por uma equação diferencial estocástica conhecida como equação de Kushner-Stratonovich, vide (WISEMAN; MILBURN, 2010; BOUTEN; HANDEL; JAMES, 2006b; BOUTEN; HANDEL; JAMES, 2006a; DOHERTY et al., 2000). A Tabela 2, reproduzida de (DOHERTY et al., 2000), apresenta um conjunto de analogias entre a dinâmica de sistemas clássicos e sistemas quânticos.

Para os sistemas dinâmicos quânticos essa equação é conhecida como equação de filtragem quântica. A nomenclatura em uso nessa área de pesquisa costuma afirmar que a equação de filtragem é uma equação para o estado condicionado do sistema, ou seja, para a estimativa do estado que se encontra condicionada aos resultados das medições que foram coletados ao longo da evolução do sistema. É também conhecida como equação de Belavkin, em homenagem a Viacheslav Belavkin, quem a primeiro deduziu em 1988. A equação (4.73) acima é uma equação de Belavkin ou equação de filtragem quântica. A relação entre o estado dinamicamente condicionado e o registro de valores medidos fica mais explícita quando escrevo que o ruído $d W$ é dado por

$$
d W=\sqrt{8 \kappa}(d y-\langle X\rangle d t)
$$


Tabela 2 - Quantum/Classical Analogies in State-Estimation

\begin{tabular}{|l|l|}
\hline \hline Classical State Estimation & Quantum State Estimation \\
\hline A Posteriori Probability Distribution & Conditioned Density Matrix \\
Kushner-Stratonovitch Equation & Non-linear Stochastic Master Equation \\
Zakai Equation & Linear Stochastic Master Equation \\
Innovation/Residual process & Quantum residuals $\left(d y-\left\langle Q+Q^{\dagger}\right\rangle d t\right)$ \\
Fokker-Planck Equation for a priori distribution & Master Equation \\
Fictitious noise to simulater KS Eq. using SDE & Fictitious noise to simulate SME using SSE \\
State Vector & Operators for system Observables \\
Process noise & Bath Noise Operators \\
Measurement noise & Meter Field Noise Operators \\
\hline \hline
\end{tabular}

A equação de Belavkin abriu ao alcance da teoria de controle de sistemas dinâmicos por realimentação, os sistemas de natureza quântica. A nova área de pesquisa inaugurada é muitas vezes denominada controle quântico por realimentação quantum feedback control. Pode-se destacar também a classe de sistemas de controle denominadasistemas de controle quântico coerente. Nessa outra categoria, se encontram sistemas dinâmicos para os quais tanto a planta quanto o controlador são sistemas quânticos. Não há adição de ruído (dinâmica incoerente) em consequência de processos de medição, pois esses são dispensáveis. A interação dinâmica entre a planta e controlador é realizada por sinais e interações de natureza quântica e não adicionam ruído à dinâmica. Os sistemas ópticos são flexíveis e adaptáveis a esta técnica, que, no entanto, é geral, vide (MABUCHI, 2014).

As redes de sistemas interconectados são descritos por dispositivos caracterizados por parâmetros $(S, L, H)$, em que $S$ é uma matriz de espalhamento (scattering matrix), $L$ é uma matriz de acoplamento que descreve como sinal se acopla aos graus de liberdade internos do dispositivo e $H$ é o Hamiltoniano que descreve a dinâmica dos graus de liberdade internos do dispositivo. Seja um dispositivo

$$
(S, L, H)=\left(\left[\begin{array}{lll}
1 & 0 & 0 \\
0 & 1 & 0 \\
0 & 0 & 1
\end{array}\right],\left[\begin{array}{cc}
\sqrt{\kappa_{1}} & a \\
\sqrt{\kappa_{2}} & a \\
\sqrt{\gamma} & \sigma
\end{array}\right], \Delta a^{\dagger} a+\theta|e\rangle\langle e|+g\left(a \sigma^{\dagger}+a^{\dagger} \sigma\right)\right)
$$

então a equação mestra que descreve seu estado é dada por

$$
\dot{\rho}=-i[H, \rho]+\left(\kappa_{1}+\kappa_{2}\right) \mathcal{D}[a] \rho+\gamma \mathcal{D}[\sigma] \rho .
$$

Há regras para a composição de circuitos interligando os dispositivos em série e em paralelo. Uma boa introdução ao assunto pode ser encontrada em Mabuchi (MABUCHI, 2014).

Os autores que desenvolveram as redes $S L H$, Nurdin, James e Doherty, inspiraramse na síntese de redes lineares (linear network synthesis) (NURDIN; JAMES; DOHERTY, 2010). 
Observação 58 (Ruído na perspectiva de Heisenberg). Na perspectiva de Heisenberg, um cuidado especial deve ser tomado na busca de equações dinâmicas para observáveis (MABUCHI, 2014), incorporando aspectos estocásticos. Em princípio, a partir da equação de Heisenberg para um observável $a$

$$
a_{t}=U_{t} a_{0} U_{t}^{\dagger}
$$

é possível obter sua forma diferencial

$$
d a_{t}=U_{t} a_{0} d U_{t}^{\dagger}+d U_{t} a_{0} U_{t}^{\dagger}+d U_{t} a_{0} d U_{t}^{\dagger}
$$

A diferencial $d U_{t}$ deve ser composta por elementos coerentes (relacionados a $d t$ ) e por elementos não coerentes (relacionados a um incremento de processo estocástico), ou seja, relacionados à evolução determinística e estocástica do sistema. No caso de equação de Heisenberg estocástica a estrutura não comutativa da mecânica quântica faz-se presente de forma não trivial. Como resultado, para tratar esse problema, uma teoria de probabilidade quântica, que generaliza a teoria de probabilidade clássica para lidar com medições de observáveis não compatíveis, foi desenvolvida, assim como uma generalização do cálculo estocástico de Itô-Stratonovich, chamado cálculo estocástico quântico. O incremento de um processo estocástico quântico na perspectiva de Heisenberg é um operador, que possui relação de comutação característica e determinados elementos quadráticos proporcionais a $d t$, além de outros diferenciais estocásticos generalizados, vide (BOUTEN; HANDEL; JAMES, 2006b; BOUTEN; HANDEL; JAMES, 2006a; MABUCHI, 2014).

Trata-se de uma teoria matemática bastante complexa. Fisicamente, as equações estocásticas na perspectiva de Heisenberg, denominadas equações de Langevin quânticas resultam da aplicação da teoria de input-output, que se baseia nas equações da eletrodinâmica quântica, vide (JACOBS, 2014; GARDINER; PARKINS; ZOLLER, 1992; WISEMAN; MILBURN, 2010).

Assim, a manipulação de operadores e de elementos diferenciais estocásticos na perspectiva de Heisenberg é mais complexa, no entanto, o valor esperado para o observável do sistema em um determinado instante deve ser único, seja ele calculado a partir de uma equação mestra estocástica, seja calculado a partir de uma equação diferencial estocástica quântica, QSDE.

Um destaque pode ser feito a partir de uma análise inicial da teoria de probabilidade quântica. Uma vez que se trata de uma generalização da teoria de probabilidade clássica, deve contê-la como um caso especial. De fato, a teoria de probabilidade quântica para uma álgebra de observáveis que comutam é equivalente à teoria de probabilidade clássica. Assim, novamente, as subalgebras de operadores auto adjuntos que comutam desempenham um papel importante na teoria de controle de sistemas dinâmicos quânticos. 
Dessa forma, nas subvariedades integrais associadas a álgebras de operadores auto adjuntos que comutam, há uma estrutura natural de probabilidade clássica e de cálculo estocástico clássico.

\subsection{Controle de Sistemas Quânticos Lineares}

Sistemas dinâmicos quânticos determinados por Hamiltonianos com termos quadráticos em operadores auto adjuntos de espectro contínuo podem ser parcialmente representados matematicamente por equações diferenciais ordinárias lineares, o que permite a aplicação de métodos clássicos de controle por meio de mecanismos de realimentação, com resultados positivos. Seguimos a apresentação de (WISEMAN; MILBURN, 2010), podem tembém ser consultados (JACOBS, 2014; JAMES; NURDIN; PETERSEN, 2007). A representação ocorre parcialmente, pois as equações diferenciais ordinárias modelam apenas o comportamento da média dos observáveis do sistema dinâmico quântico. A representação não poderia ser completa, pois os sistemas quânticos representados possuem dimensão infinita e o espaço de estados de sistemas de equações diferenciais ordinárias possui dimensão finita.

De forma a se obter modelos descritos por equações diferenciais lineares, dado um sistema descrito por $N$ pares de variáveis canonicamente conjugadas $q_{m}$ e $p_{m}$, arranjam-se os observáveis da seguinte forma:

$$
\mathbf{x}^{T}=\left(q_{1}, p_{1}, q_{2}, p_{2}, \cdots, q_{N}, p_{N}\right)
$$

os termos $q_{i}$ e $p_{i}$ que compõem o vetor de estados representam a média dos respectivos observáveis quânticos.

As relações de comutação podem ser escritas como $\left[x_{m^{l}}, x_{m}\right]=i \hbar \Sigma_{m^{l}, m}$ onde $\Sigma$ é uma matriz anti-simétrica formada por blocos diagonais, sendo representada por:

$$
\Sigma=\oplus_{1}^{N}\left(\begin{array}{cc}
0 & 1 \\
-1 & 0
\end{array}\right)
$$

Sendo $V$ a matriz de covariância, como consequência do princípio da incerteza pode-se escrever que:

$$
V+i \hbar \frac{\Sigma}{2} \geq 0
$$

A partir das definições colocadas acima, pode-se mostrar que existem matrizes $A$, $B, C, E$ e $G$ tais que a dinâmica das variáveis de estado do sistema quântico obedeçam as seguintes equações diferenciais estocásticas lineares (JAMES; NURDIN; PETERSEN, 2007; WISEMAN; MILBURN, 2010):

$$
d \mathbf{x}=A \mathbf{x} d t+B \mathbf{u}(t)+E d \mathbf{v}_{p}(t)
$$




$$
\mathbf{y} d t=C \mathbf{x} d t+d \mathbf{v}_{m}(t)
$$

Para um hamiltoniano quadrático em $\mathbf{x}$, do tipo:

$$
H=\frac{1}{2} \mathbf{x}^{T} G \mathbf{x}-\mathbf{x}^{T} \Sigma B \mathbf{u}(t)
$$

O teorema de Ehrenfest é importante para entender a composição das matrizes do sistema linear a partir da definição do Hamiltoniano, vide (RAMAKRISHNA et al., 1995; SAKURAI, 1994). É igualmente importante a utilização das relações de comutação canônicas e da perspectiva de Heisenberg, vide (JACOBS, 2014). A utilização das relações de comutação fica facilitada a partir de uma proposição encontrada em (SAKURAI, 1994, pp. 84-85).

A matrizes que definem o modelo quântico apresentado acima podem ser diretamente deduzidas das equações diferenciais gerais que representam a evolução temporal de um sistema quântico sujeito a perturbações estocásticas causadas por processos de medição.

A partir da terminologia definida, pode-se mostrar que existe uma noção de controlabilidade para sistemas quânticos que, ainda que não seja equivalente à noção de controlabilidade por estado puro, representa a possibilidade de se definir em $\mathbb{R}^{2 N}$ o ponto de equilíbrio ou centroide do sistema, em função da escolha de $u(t)$ exatamente como ocorre em sistemas de controle lineares multivariáveis. O critério de controlabilidade mencionado expressa-se em função das matrizes $A$ e $B$ exatamente como a matriz de controlabilidade de Kalman, para a qual se deve obter posto completo. A única diferença se dá pela presença da matriz $\Sigma$, vide (WISEMAN; MILBURN, 2010) seção 6.3.2.

Em termos de álgebras de Lie o conceito se expressa da seguinte forma (WISEMAN; MILBURN, 2010, pp. 318-320): “Um sistema quântico linear é controlável se, e somente se, a álgebra de Lie gerada pelo Hamiltoniano livre e pelos Hamiltonianos de controle inclui um conjunto completo de observáveis". Devemos notar que Wiseman e Milburn utilizam uma definição de conjunto completo de observáveis que é diferente da definição utilizada por Prugovecki (PRUGOVECKI, 1981). Wiseman e Milburn definem um conjunto completo de observáveis como uma base para o espaço vetorial dos operadores lineares auto adjuntos que agem no espaço de Hilbert do sistema de interesse, já Prugovecki define conjunto completo como uma família de operadores capazes de gerar uma álgebra abeliana maximal, ou seja, uma álgebra de operadores que comutam, que é uma subalgebra propriamente contida na álgebra dos operadores auto adjuntos, que contém em geram operadores que não comutam entre si.

Entretanto, essa discussão sobre a forma utilizada para expressar o conceito de controlabilidade não interfere no resultado. A média dos observáveis do sistema quântico 
poderá ser arbitrariamente modificada no espaço $\mathbb{R}^{2 N}$ a partir dos parâmetros de controle, se, e somente, a matriz de controlabilidade de Kalman tiver posto completo. Ressalta-se mais uma vez que, a partir dessa noção de controlabilidade, não decorre que seja possível preparar um estado arbitrário do sistema quântico.

Em contraposição ao conceito apresentado e discutido acima encontramos em (WISEMAN; MILBURN, 2010) o conceito de controlobilidade de operadores, expresso pela definição: "Um sistema quântico é controlável por operadores se, e somente se, a álgebra de Lie gerada pelos Hamiltonianos do sistema de controle é igual a $\mathcal{D}(\mathbb{H})$, ou seja, ao conjunto de operadores auto adjuntos que agem em de $\mathbb{H}$ ". A referência citada pelo autores para sustentar essa definição, no entanto, analisa conceitos de controlabilidade em dimensão finita (RAMAKRISHNA et al., 1995). Em dimensão finita, tendo em vista a correspondência entre álgebras e grupos de Lie realizada por meio da função exponencial, essa definição faz sentido. No entanto, em dimensão infinita surgem os problemas de domínio relacionados aos operadores não limitados e também surgem problemas analíticos relacionados às propriedades de convergência dos espaços de dimensão infinita e dos espaços de operadores lineares que neles agem, vide (REED; SIMON, 1980; PRUGOVECKI, 1981; HUANG; TARN, 1983; WU; TARN; LI, 2006; KARWOWSKI; VILELA-MENDES, 2004; VILELA-MENDES, 2009; VILELA-MENDES; MANIKO, 2011).

Prosseguindo a analogia entre os sistemas de controle clássicos e quânticos, é possível obter um controlador LQG quântico que funcione a partir dos dados do vetor de saída do sistema $\mathbf{y}$ e que, com base em um estimador do estado $\mathbf{x}$ do sistema, oferece a melhor estratégia de controle $\mathbf{u}(t)$ a partir de uma determinada função de custo (WISEMAN; MILBURN, 2010; NURDIN; JAMES; PETERSEN, 2009).

\subsection{Simulações Numéricas}

\subsubsection{Spin Squeezing via Realimentação}

As propriedades de um sistema de $\mathrm{N}$ qubits ( $\mathrm{N}$ sistemas quanticos bidimensionais) podem ser representadas convenientemente por um sistema quântico de spin $=\mathrm{N} / 2$. Assim, uma população de $\mathrm{N}$ atomos com spin 1/2 ou de $\mathrm{N}$ atomos com 1 nível de energia excitado, pode ser descrita, para todos os fins, por essa metodologia. Assim procedendo, tomaremos a seguir um sistema de $\mathrm{N}$ partículas para simular tanto em malha aberta com medição de um observável quanto em malha fechada. Escolhemos $\mathrm{N}=10$ para o sistema com medição para aliviar o custo computacional e $\mathrm{N}=40$ para o sistema em malha fechada. Em malha aberta o sistema é simulado por uma equação mestra estocástica e em malha fechada por uma equação mestra determinística, vide (WISEMAN; MILBURN, 2010).

As simulações em malha aberta com medição foram realizadas com Hamiltoniano 
Figura 5 - Sistema incorporando medição do observável $J_{z}$.
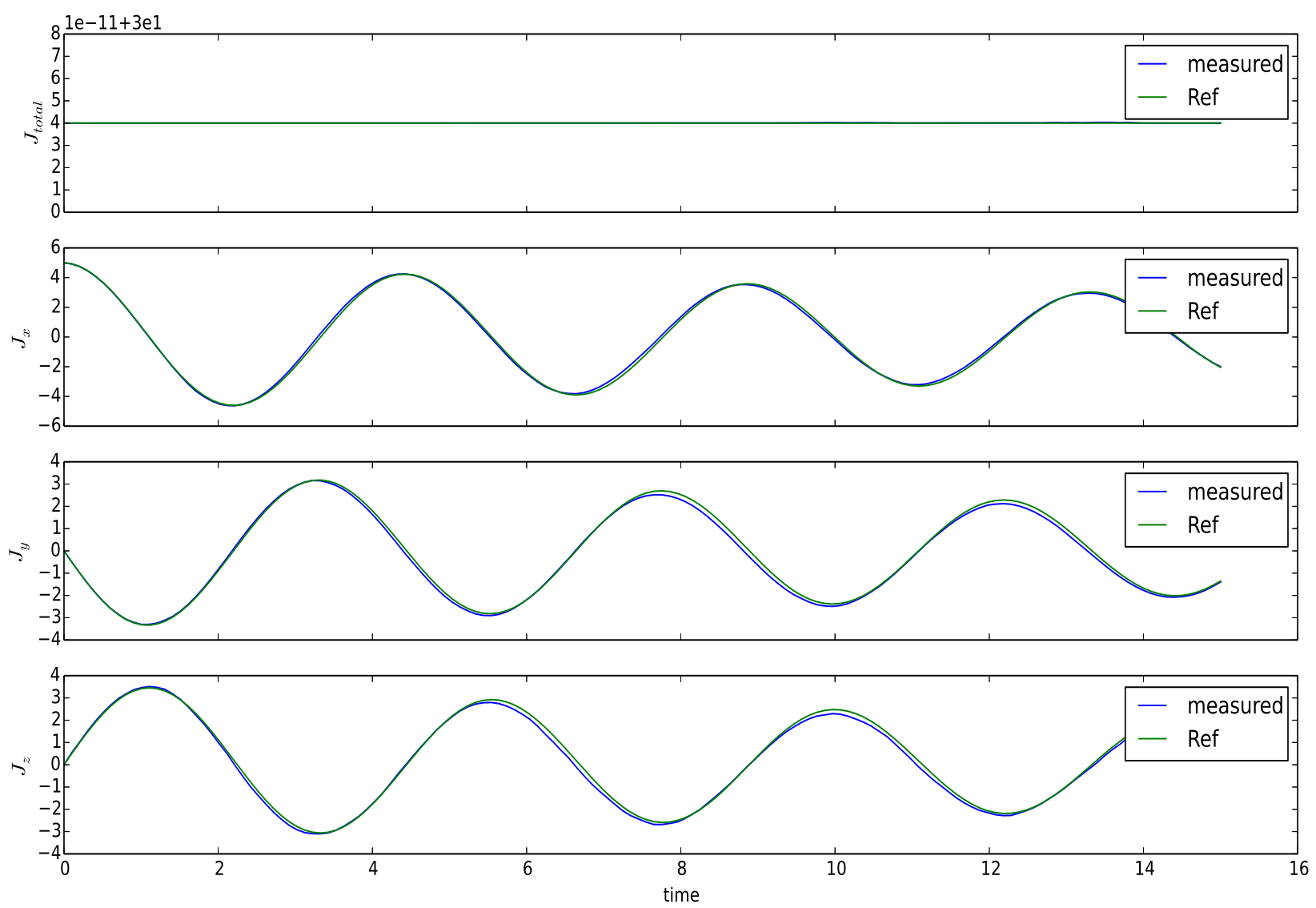

$H=J_{z}+J_{y}$. A equação mestra estocástica que modela a dinâmica do sistema com medição é

$$
d \rho_{c}=-i\left[H, \rho_{c}\right] d t+M \mathcal{D}\left[J_{z}\right] \rho_{c} d t+\mathcal{D}\left[J_{z}\right] \rho_{c} d t+\sqrt{M} \mathcal{H}\left[J_{z}\right] \rho_{c} d W
$$

Na Figura 5, podemos observar a dinâmica do sistema em malha aberta com medição. É possível notar um amortecimento das oscilações do momento angular, causado pelo termo dissipativo da equação mestra. Observa-se também que os valores esperados dos observáveis calculados a partir dos dados de medição são bastante próximos dos valores esperados reais utilizados como referência.

Na Figura 6, são mostrados os dados referentes ao processo de medição. A curva em preto representa o valor esperado real do observável e a curva em vermelho representa o valor esperado para o observável medido, calculado pela média das 300 trajetórias simuladas. Nota-se que o sinal medido acompanha bem o sinal de referência, no entanto, é bastante irregular e possui frequências altas em seu espectro.

Embora o valor medido apresente irregularidades, não observamos o mesmo fenômeno a partir da análise dos dados obtidos da simulação da equação mestra estocástica. 
Figura 6 - Sinal obtido pela simulação de medição do observável $J_{z}$.

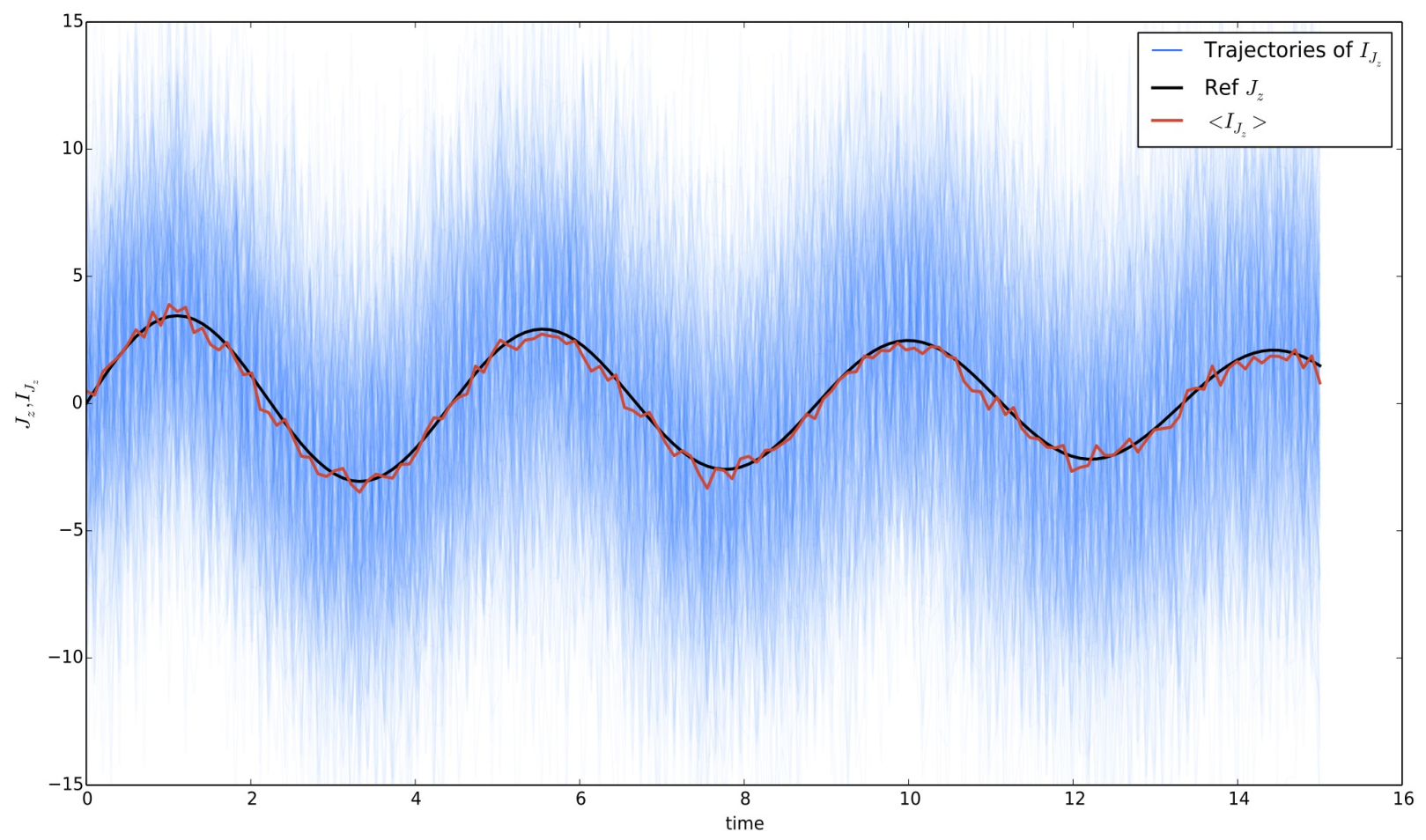

Figura 7 - Aparato experimental.

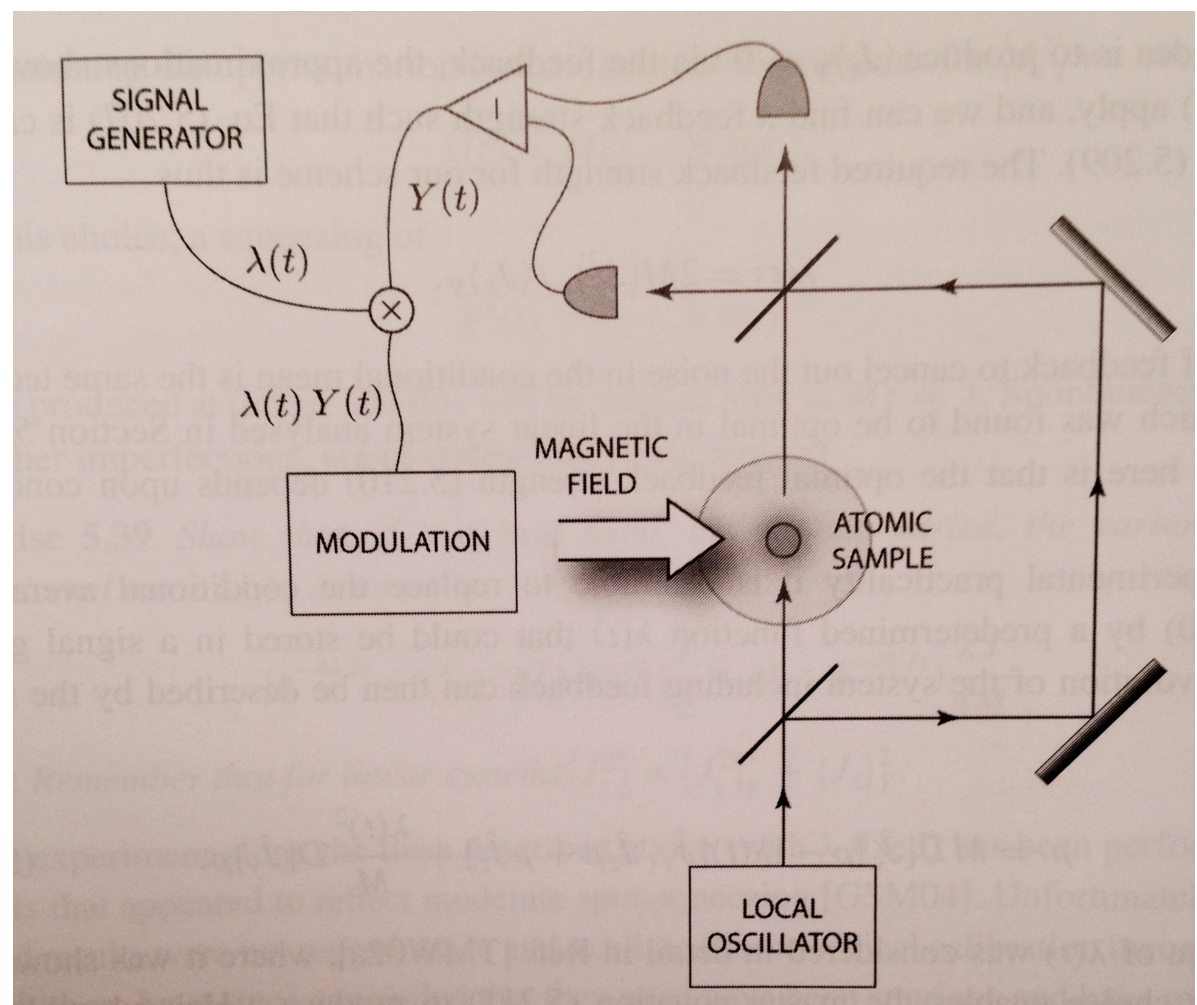


Figura 8 - Valor esperado para os observáveis $J_{x}, J_{x}^{2}, J_{y}^{2}$ e $J_{z}^{2}$, no sistema com realimentação.
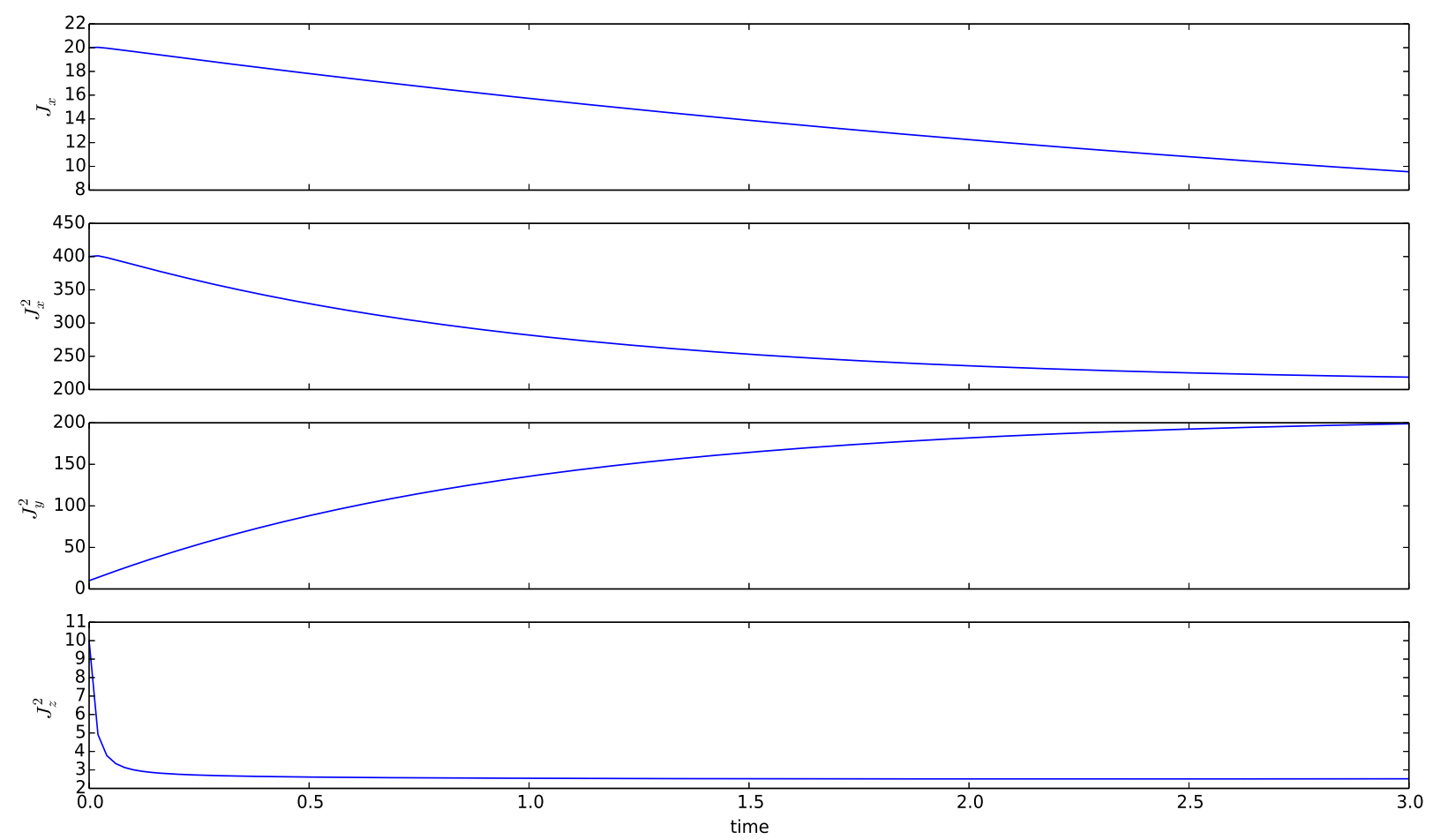

Isso ocorre, por que o incremento do processo de Wiener, $d W$, possui valor esperado nulo. Portanto, o último termo da equação mestra estocástica não interfere no cálculo das médias e os valores obtidos são os mesmos que seriam obtidos para uma equação mestra determinística.

A Figura 7 ilustra esquematicamente a configuração experimental que é simulada pelos modelos matemáticos apresentados nesta seção. Para representar o sistema em malha fechada, realimentado para criar as condições para implementar squeezing, é possível utilizar uma equação mestra determinística

$$
\dot{\rho}=\frac{\lambda(t)}{2 i}\left[J_{z} J_{y}+J_{y} J_{z}, \rho\right]+\mathcal{D}\left[\sqrt{M} J_{z}-i \lambda(t) / \sqrt{M}\right] \rho
$$

onde se deve notar que o Hamiltoniano livre é nulo. O primeiro termo é dado pela parte conservativa da dinâmica da realimentação, em conjunto com a medição, enquanto o segundo termo agrega a dinâmica dissipativa.

As Figuras 8 e 9 ilustram os resultados obtidos a partir da simulação do sistema em malha fechada. Pode-se notar uma diminuição da incerteza na direção z e uma diminuição do parâmetro de squeezing. Ressaltamos que, para simular o sistema a partir das funções da biblioteca QuTiP, utilizamos um estado inicial com valor de $J_{x}$ próximo do valor máximo, mas não no valor máximo, para evitar instabilidade numéricas no código. 
Figura 9 - Valor esperado para o observável $J_{x}$, desvio-padrão de $J_{x}, J_{y}$ e $J_{z}$ e parâmetro de squeezing, $\zeta$, no sistema com realimentação.

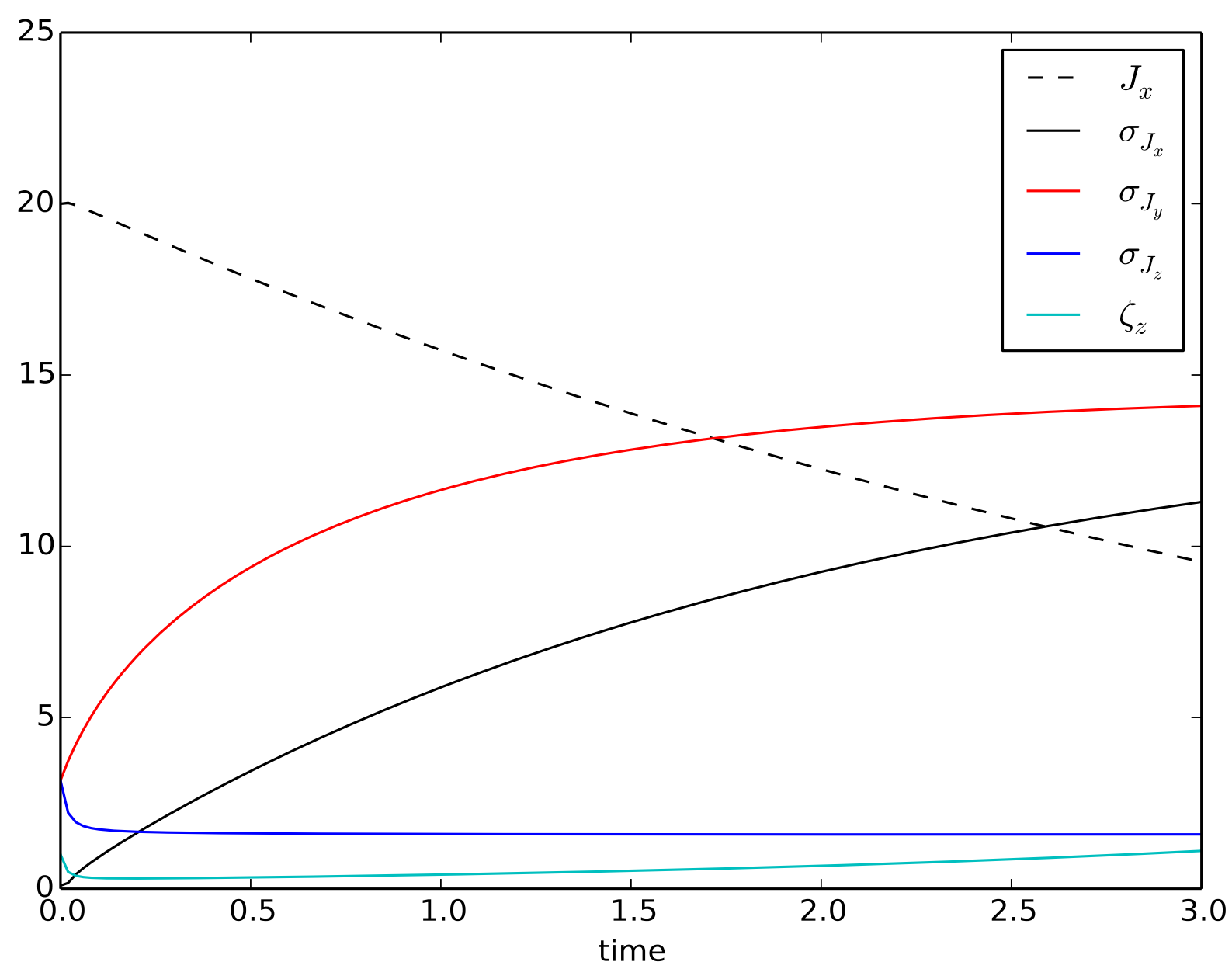

Adicionamos também que o sistema em feedback é bastante sensível a modificações do parâmetro de realimentação $\lambda(t)$, tornando-se facilmente instável.

\subsubsection{Controle de posição}

Apresentamos nesta seção, resultados de simulações numéricas realizadas a partir de um modelo de sistema de controle quântico analisado em (DOHERTY et al., 2000).

O Hamiltoniano que define a dinâmica livre é não linear. O potencial descrito pela função a ser simulada, após a definição numérica das constantes $A$ e $B$, possui dois pontos de equilíbrio estável, $\{-3,3\}$, e a origem como ponto de equilíbrio instável.

$$
H=\frac{1}{2} p^{2}-A x^{2}+B x^{4}
$$

A dinâmica do sistema sujeito a ruído térmico a uma intensidade proporcional a 
$\beta$ e a medição contínua do observável posição a uma intensidade $k$ é dada pela equação mestra estocástica:

$$
d \rho_{c}=-i\left[H+H_{f b}, \rho_{c}\right] d t+2 \beta \mathcal{D}[x] \rho_{c} d t+2 k \mathcal{D}[x] \rho_{c} d t+\sqrt{2 k} \mathcal{H}[x] \rho_{c} d W
$$

O sinal referente à medida de posição é descrito por $I(t)=\dot{Q}$, onde

$$
d Q(t)=\langle x\rangle d t+\frac{1}{\sqrt{8 k}} d W
$$

vide (DOHERTY; JACOBS, 1999; JACOBS; STECK, 2006; JACOBS, 2014).

A partir da equação mestra estocástica do sistema é possível obter estimativas para as médias dos observáveis posição e momento linear, assim como para sua matriz de covariância: ${ }^{16}$

$$
\begin{aligned}
d\langle x\rangle & =\langle p\rangle d t+2 \sqrt{2 k} V_{x} d V \\
d\langle p\rangle & =-4 B\langle x\rangle^{3} d t+2 A\langle x\rangle d t-12 B(x) V_{x} d t+2 \sqrt{2 k} C d V+u d t \\
\dot{V}_{x} & =2 C-8 k V_{x}^{2} \\
\dot{V}_{p} & =-24 B\langle x\rangle^{2} C+4 A C-24 B C V_{x}+2(k+\beta) \hbar^{2}-8 k C^{2} \\
\dot{C} & =V_{p}-12 B\langle x\rangle^{2} V_{x}+2 A V_{x}+12 B V_{x}^{2}-8 k C V_{x}
\end{aligned}
$$

A partir das estimativas para o estado do sistema dinâmico, é possível definir uma estratégia de controle por realimentação de estados, dada em (DOHERTY et al., 2000) por $u=u_{0}+u_{1}+u_{2}$, onde

$$
\begin{aligned}
& u_{0}=2 A\langle x\rangle-4 B\langle x\rangle^{3} \\
& u_{1}=-\tilde{u}\left(\langle x\rangle-x_{0}\right) \\
& u_{2}=-(\sqrt{2 \tilde{u}+\Gamma})\left(\langle p\rangle-p_{0}\right) \\
& \tilde{u}=\partial_{\langle x\rangle} u_{0}+\sqrt{\left[\partial_{\langle x\rangle} u_{0}\right]^{2}+\Gamma} .
\end{aligned}
$$

Os mesmos valores numéricos indicados no artigo foram utilizados nas simulações.

Inicialmente apresentamos nas Figuras 10, 11 e 12 a dinâmica do sistema livre do sistema com medição contínua do observável posição. Devemos ressaltar que os gráficos fornecem uma estimativa para o estado do sistema dinâmico quântico real e que a estimativa assume a hipótese de que o estado do sistema é aproximadamente gaussiano.

Assim, não estamos realizando diretamente uma simulação por trajetórias quânticas nem da equação mestra estocástica nem da equação de Schrödinger estocástica

\footnotetext{
16 Assume-se, na definição das equações dinâmicas do estimador, que o estado do sistema dinâmico
} quântico é aproximadamente gaussiano. 
equivalente. Em (DOHERTY et al., 2000), os autores simulam trajetórias a partir da SSE equivalente e conseguem estabelecer uma média que denominam "verdadeira". Pela compararação entre os dados estimados com base na hipótese de estado gaussiano e os dados simulados numericamente para as trajetórias quânticas, o estimador gaussiano é bastante eficiente, conforme os resultados apresentados na referência. Assim, é possível utilizar, na solução de problemas de controle quânticos, a técnica de testar as funções que definem a estratégia de controle no sistema clássico equivalente, assumindo que a distribuição de probabilidade nos estados do sistema é gaussiana. A validação da estratégia de controle poderá ser realizada a posteriori, tendo em vista que seu custo computacional é muito maior.

É interessante notar que em dinâmica livre, o ruído introduzido pelo processo de medição e pelas interações térmicas com o ambiente é suficiente para para retirar o sistema de um ponto de equilíbrio estável, vide Figura 10.

Nas Figuras 13, 14 e 15 são apresentados os resultados de simulações para o sistema com realimentação. É interessante observar a dinâmica estimada para as variáveis $x$ e $p$, no periodo em que o sistema de controle está realizando a transição entre os setpoints. Observemos que o setpoint para o momento linear é nulo, para que o esforço de controle possa ser minimizado. Quando, ao realizar a transição, a variável $p$ possui se encontra com valores baixos, o sistema de controle tem mais dificuldade de movimentar a variável de estado $x$. Este fenômeno pode ser bem observado na Figura 15, na transição realizada em $t=5$. Podemos relacionar esta dinâmica à forma do termo $u_{2}$ da função de controle.

Nas Figuras 16, 17 e 18 apresentamos trajetórias de simulação para o sistema de controle com uma nova função de controle. Com o objetivo de cancelar a dinâmica do potencial, dado pelo termo $2 A\langle x\rangle-4 B\langle x\rangle^{2}$, mudamos o sinal do termo $u_{0}$ na função de controle. 
Figura 10 - Amostra de trajetória para uma realização de ruído arbitrária, dinâmica livre.
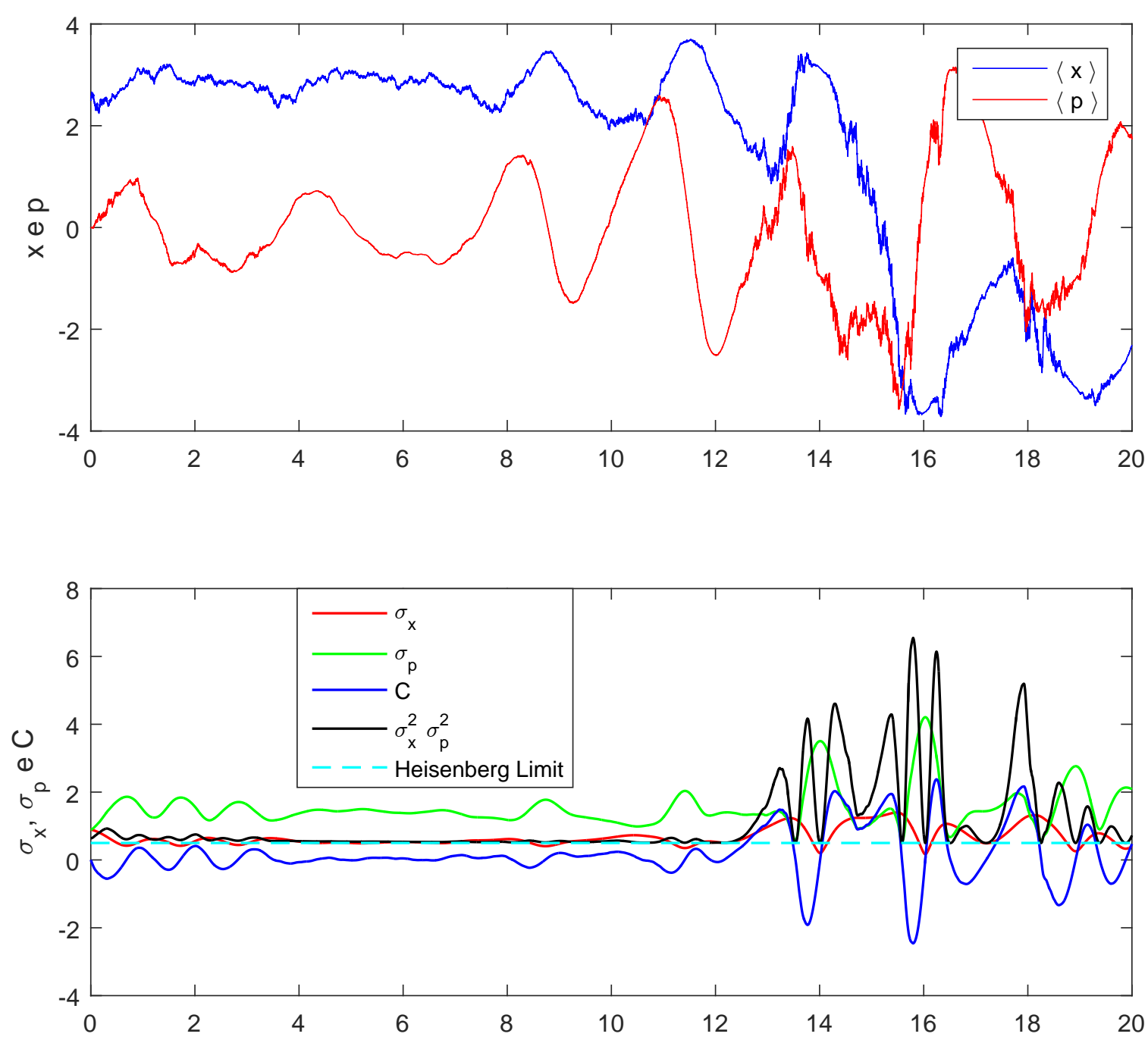
Figura 11 - Amostra de trajetória para uma realização de ruído arbitrária, dinâmica livre.
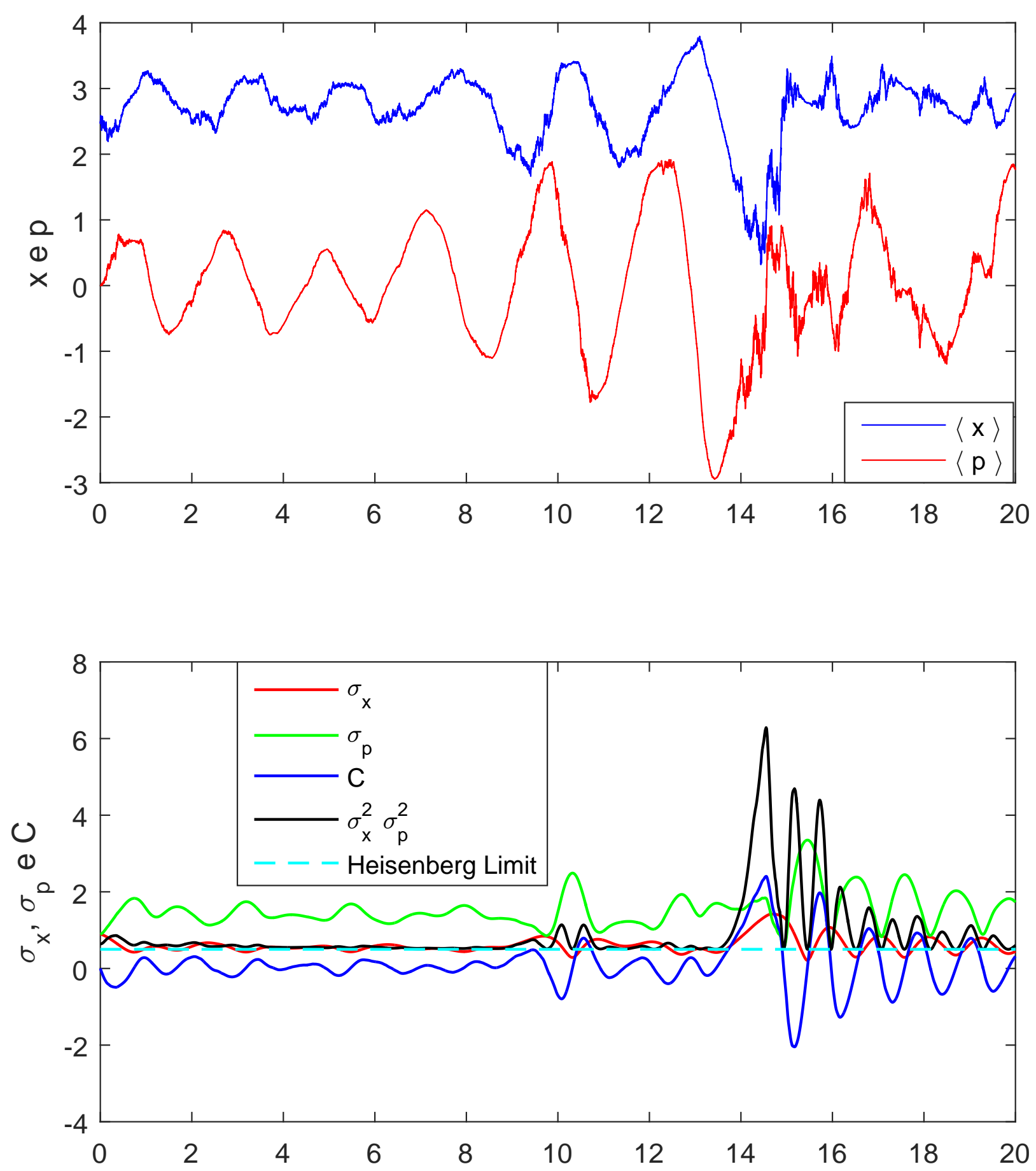
Figura 12 - Amostra de trajetória para uma realização de ruído arbitrária, dinâmica livre.
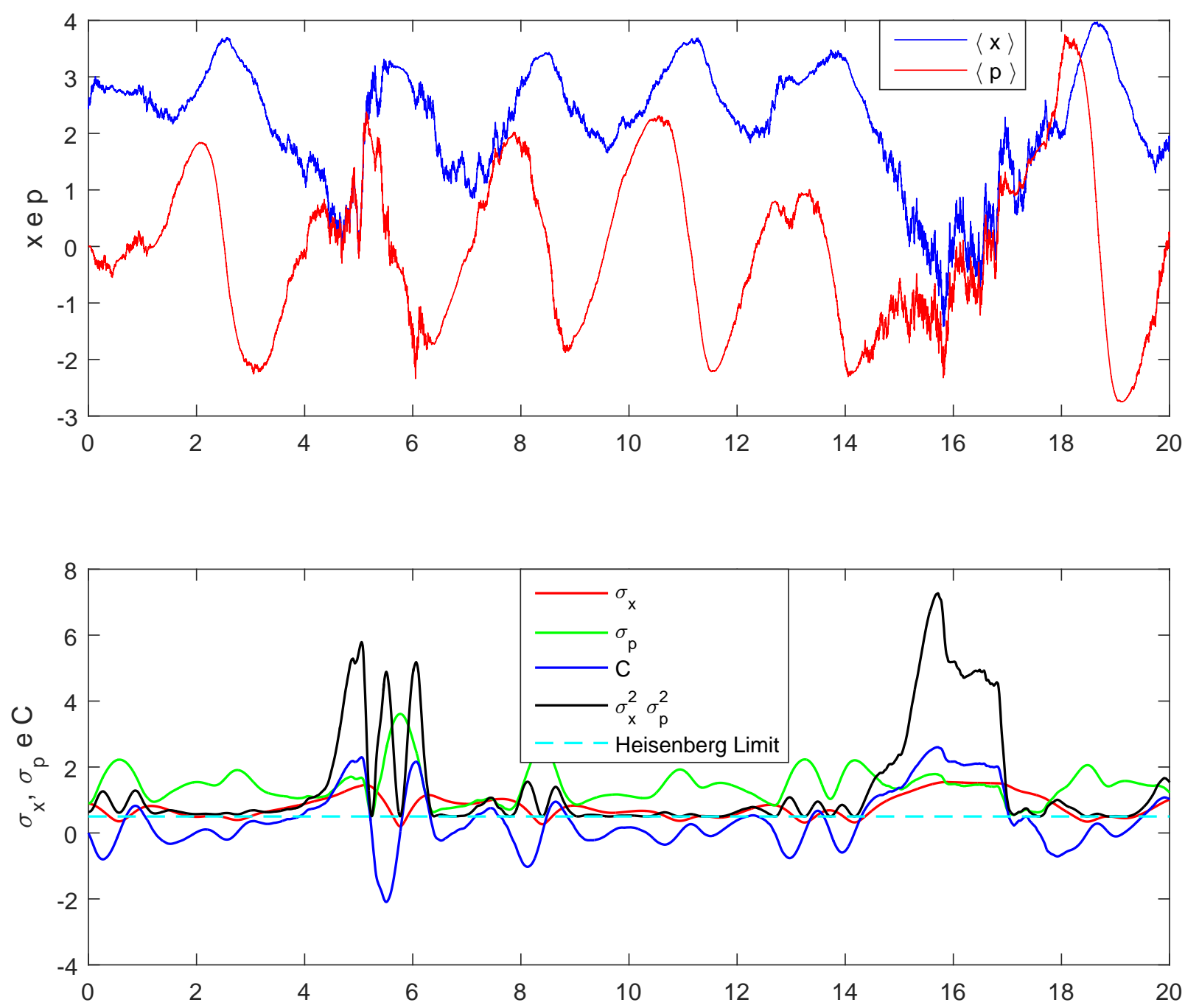
Figura 13 - Amostra de trajetória para uma realização de ruído arbitrária, dinâmica controlada.
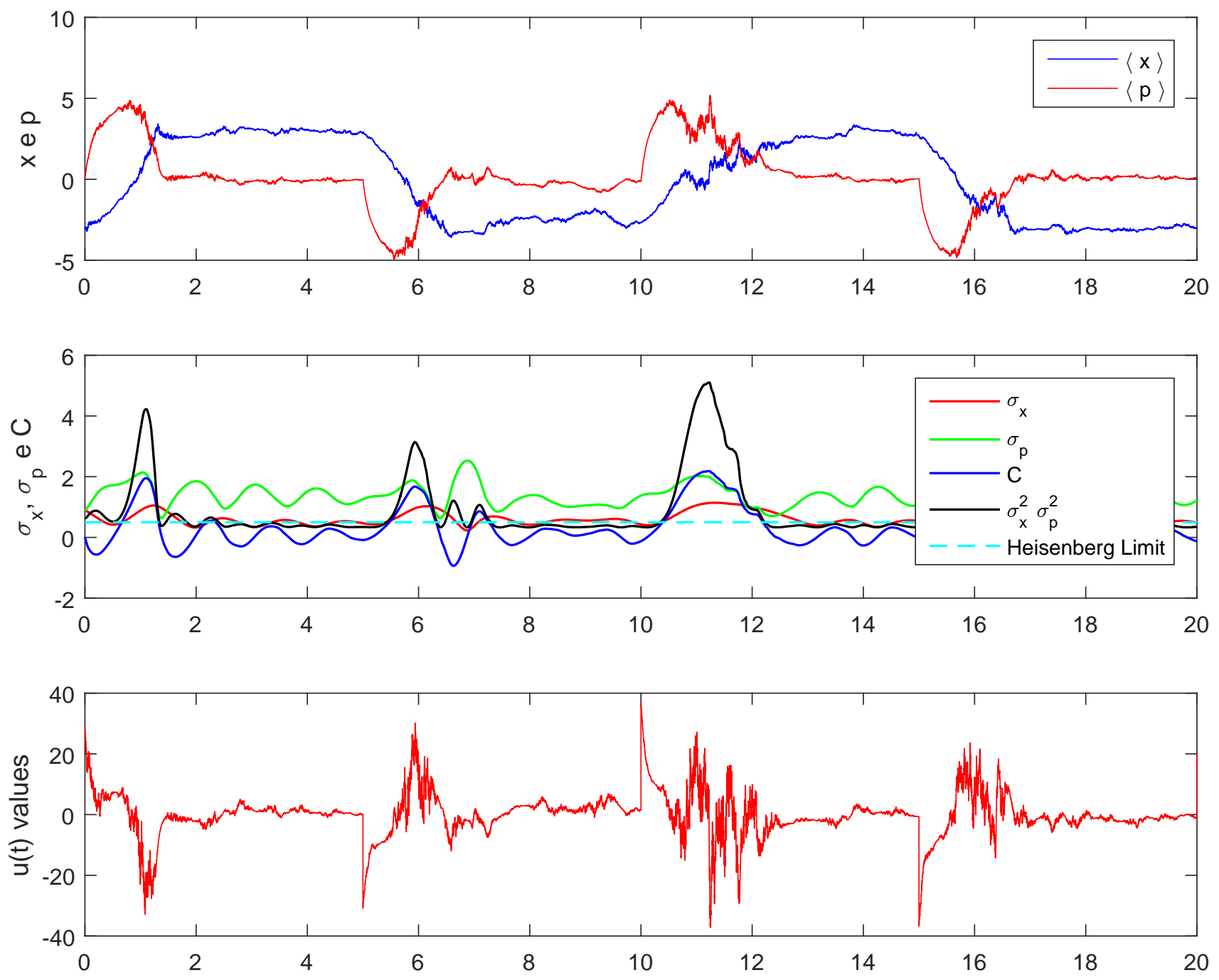
Figura 14 - Amostra de trajetória para uma realização de ruído arbitrária, dinâmica controlada.
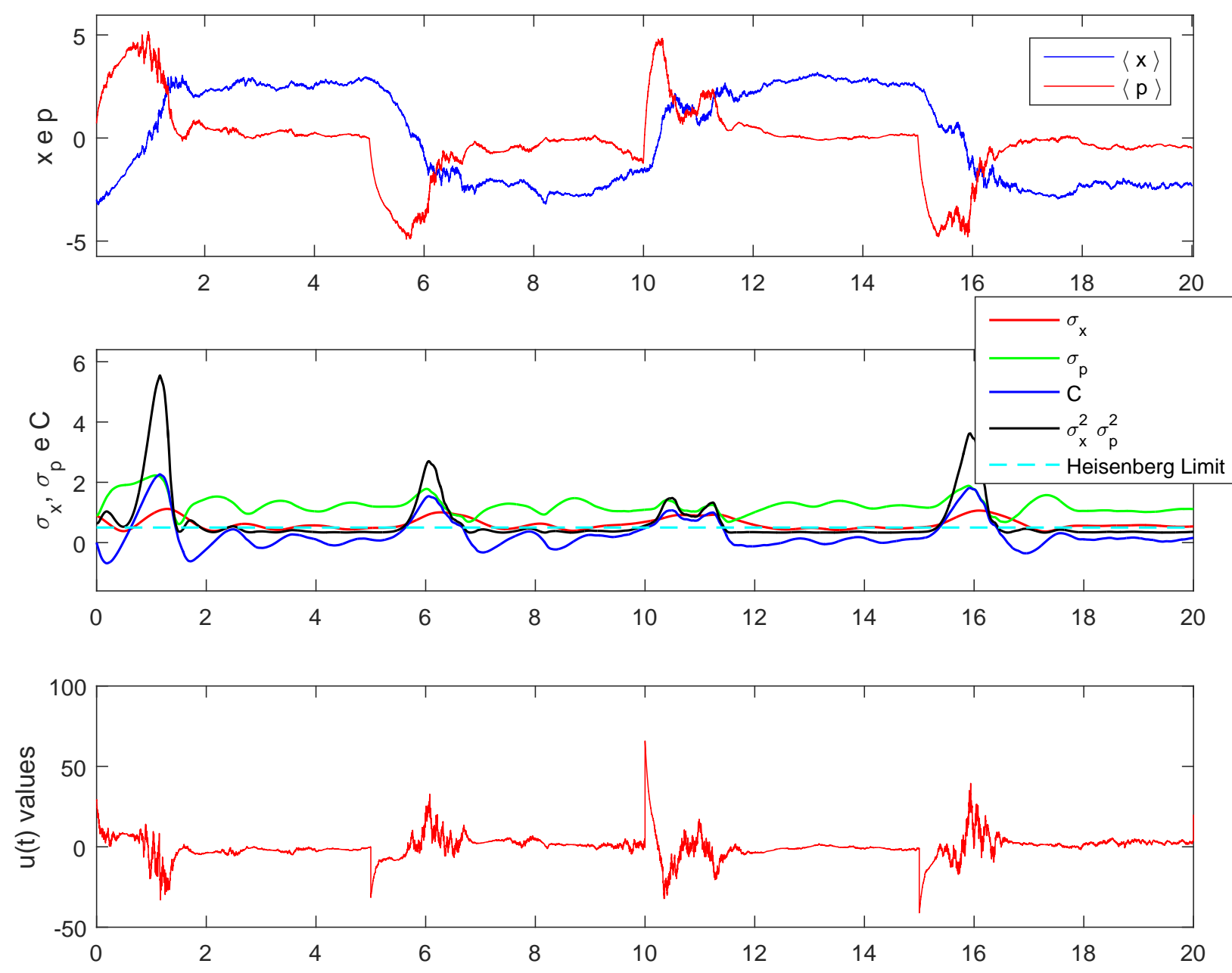
Figura 15 - Amostra de trajetória para uma realização de ruído arbitrária, dinâmica controlada.
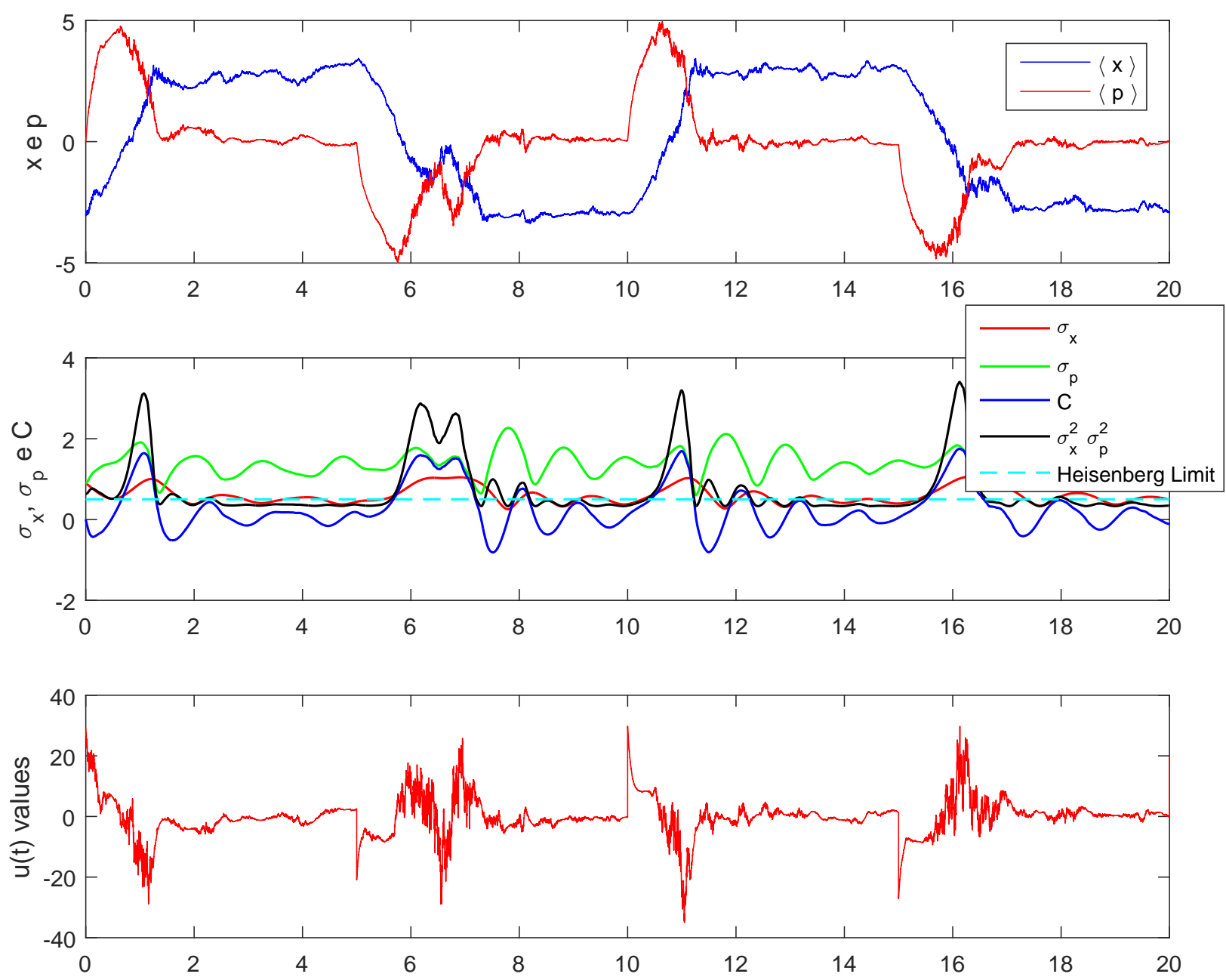
Figura 16 - Amostra de trajetória para uma realização de ruído arbitrária, dinâmica controlada e função de controle modificada.
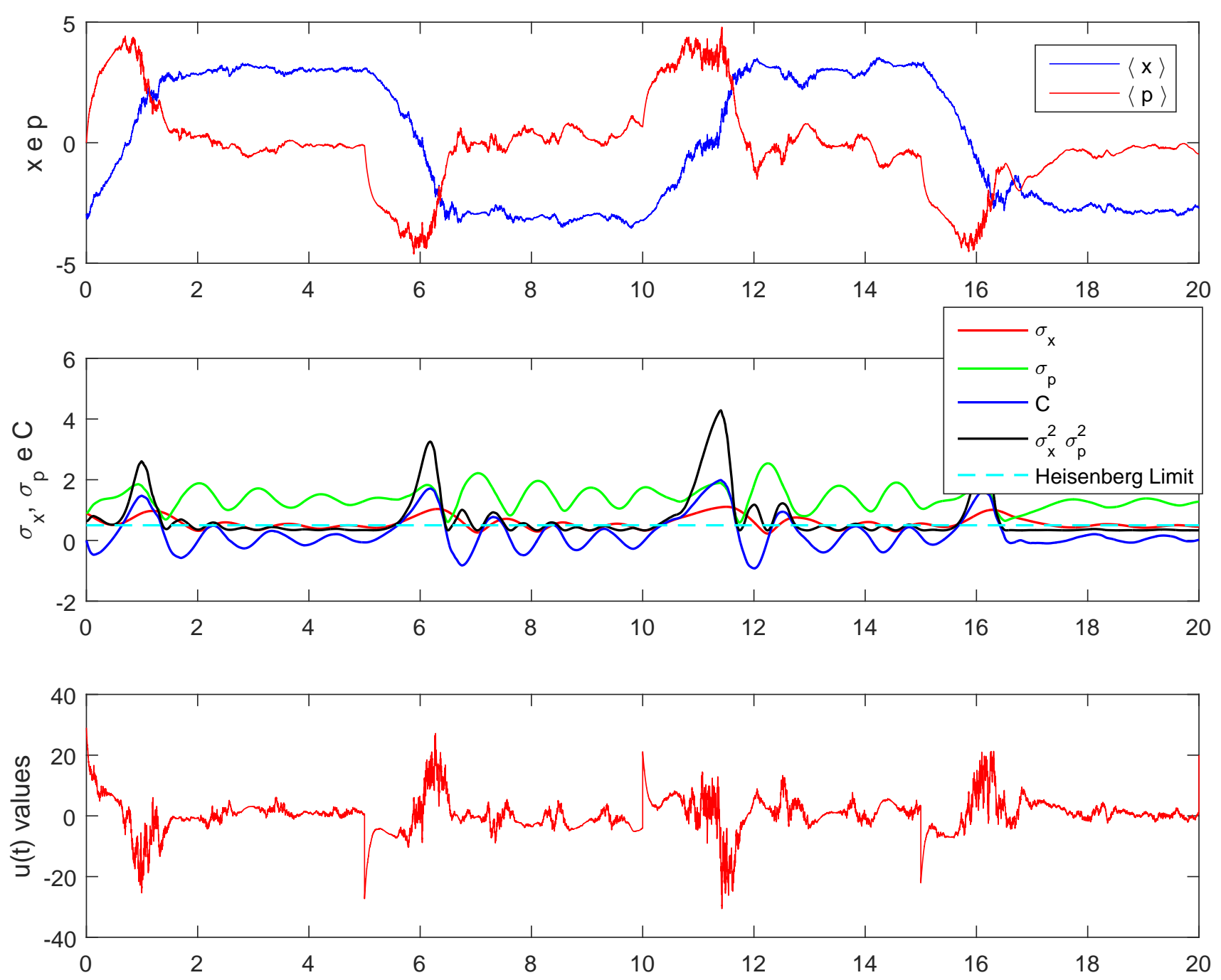
Figura 17 - Amostra de trajetória para uma realização de ruído arbitrária, dinâmica controlada e função de controle modificada.
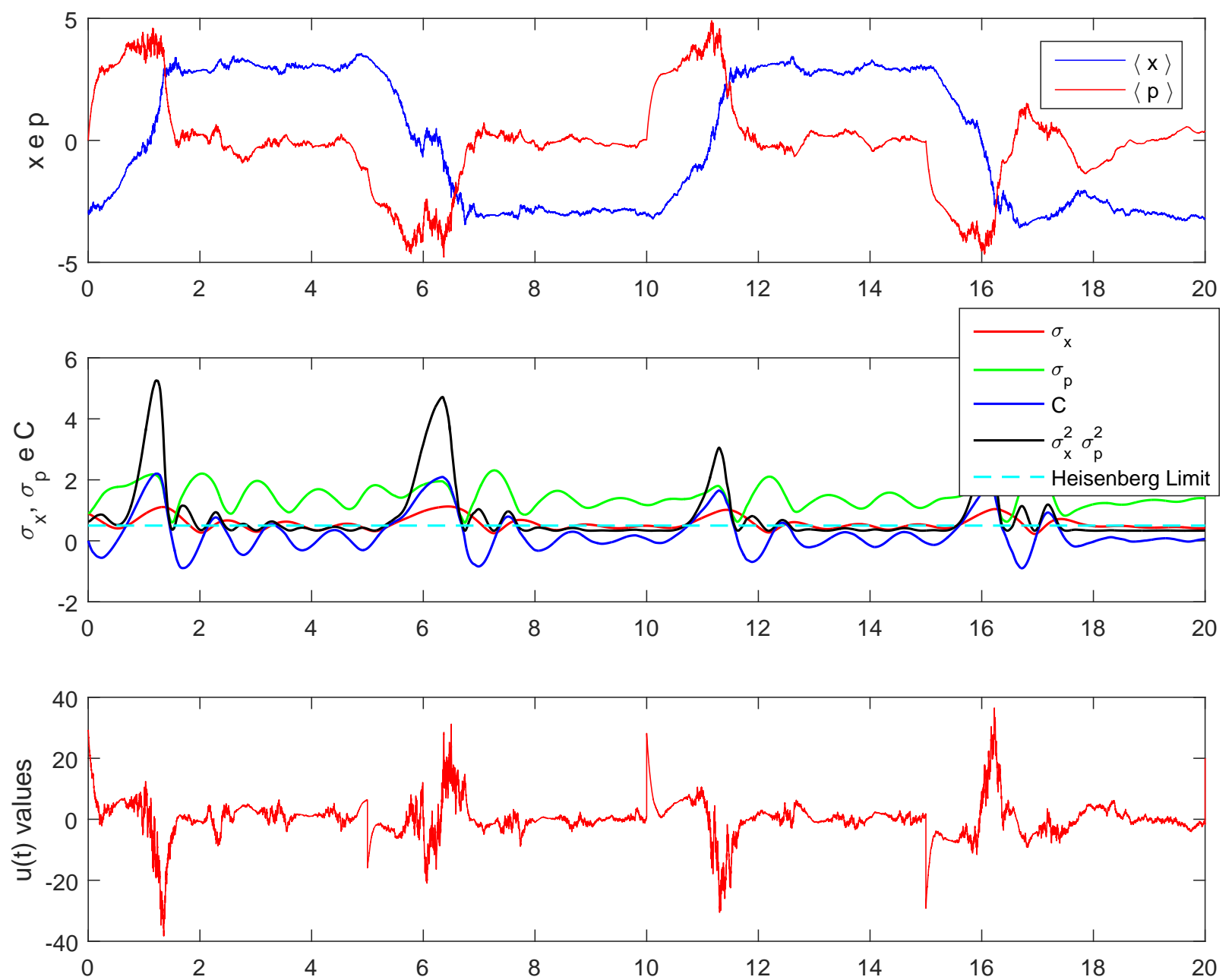
Figura 18 - Amostra de trajetória para uma realização de ruído arbitrária, dinâmica controlada e função de controle modificada.
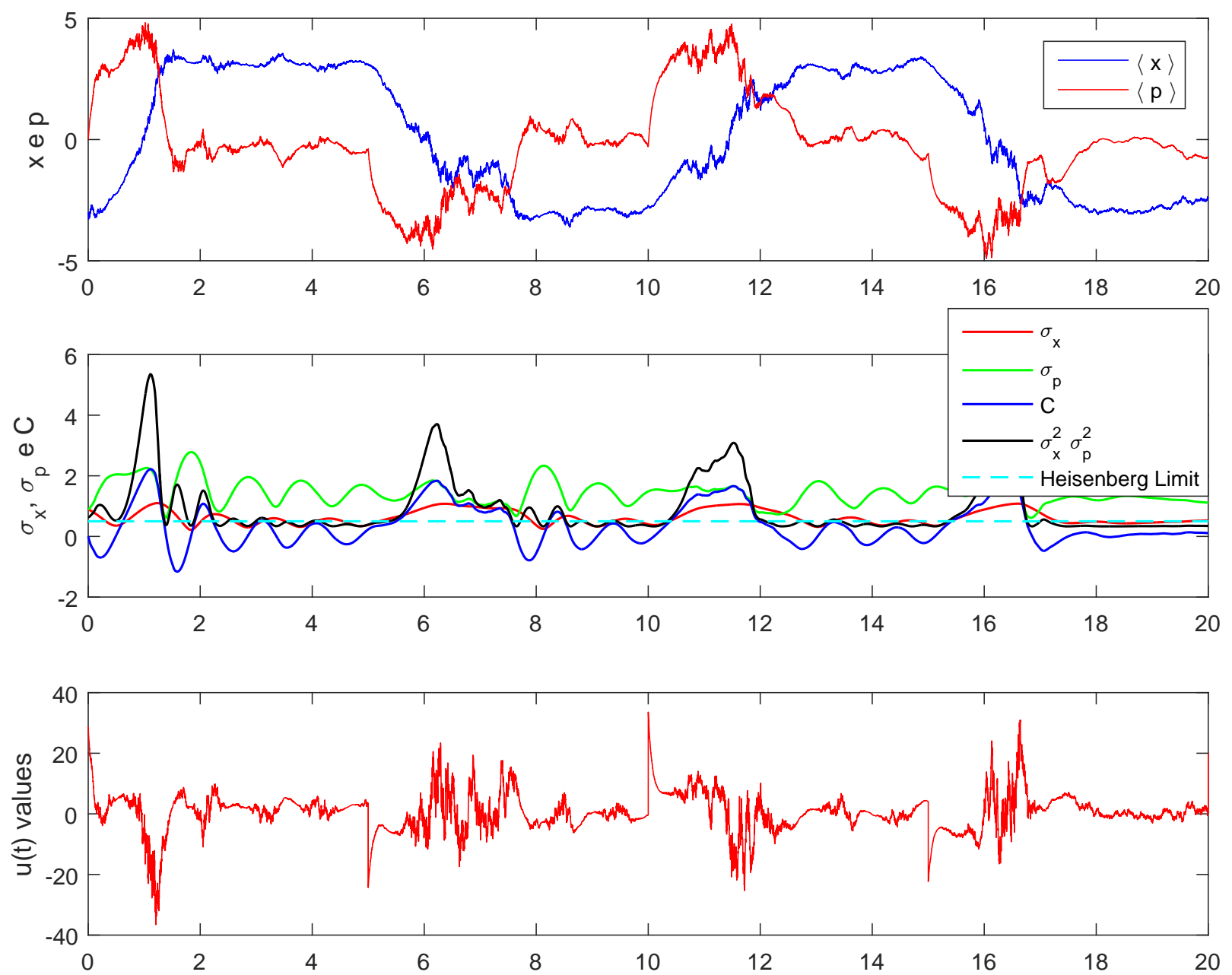


\section{Resultados e Discussão}

\subsection{Incerteza e Medição}

A relação entre o princípio da incerteza e os processos de medição de sistemas quânticos é bastante próxima. A natureza estocástica da dinâmica de sistemas quânticos que incorporam medição tem sua origem na relação de Heisenberg, que estabelece também um limite para a aquisição de informação a respeito de um estado de sistemas dinâmicos quânticos. Apresentamos abaixo uma discussão do princípio da incerteza e de sua relação com a medição.

O princípio da incerteza é um resultado fundamental na teoria de sistemas quânticos. Fisicamente, estabelece um limite fundamental máximo para a quantidade de informação que um observador pode extrair de um sistema quântico por meio de uma álgebra de operadores auto-adjuntos que age em um espaço de Hilbert adequado, que contém o estado do sistema. O princípio de Heisenberg generalizado, para operadores de uma álgebra de Lie se escreve como, vide (SAKURAI, 1994; BRAGINSKY; KHALILI, 1995) ${ }^{1}$ :

$$
\left\langle(\Delta A)^{2}\right\rangle\left\langle(\Delta B)^{2}\right\rangle \geq \frac{1}{4}|\langle[A, B]\rangle|^{2}
$$

No caso de estarmos restritos a um conjunto de operadores que comutam, que formam uma algebra abeliana, este limite fundamental se expressa trivialmente pois, como os operadores comutam, o lado direito da equação será nulo e não há, nesse caso particular, um limite finito para o produto das variâncias associadas a medição de quaisquer dois observáveis dessa álgebra. O comportamento esperado é qualitativamente o mesmo tanto para medições realizadas nesta álgebra quanto para medições realizadas em um sistema clássico qualquer.

Do ponto de vista matemático, o princípio da incerteza relaciona de modo não trivial as teorias de medida e integração, as álgebras de operadores em espaços de Hilbert e o teorema espectral. Vide também a teorema de Freudenthal que generaliza o teorema de Radon-Nikodym em teoria da medida e possui o teorema espectral como um corolário.

$\overline{1}$ Apresenta-se em (CLERK, 2014) uma expressão para o princípio da incerteza na forma

$$
\left\langle(\Delta A)^{2}\right\rangle\left\langle(\Delta B)^{2}\right\rangle \geq \frac{1}{4}|\langle[A, B]\rangle|^{2}+\frac{1}{4}|\langle\{A, B\}\rangle|^{2} \quad,
$$

citando como refência (GOTTFRIED, 1966). Não há inconsistência entre as desigualdades, tendo em vista que em (GOTTFRIED, 1966), deixa-se claro que o limite inferior, dado pela equação 5.2, é atingido para um determinado estado, que é específico aos observáveis $A$ e $B$. Para estados arbitrários, devemos tomar a expressão dada por (CLERK, 2014), equação 5.1. 
De todo modo, a não comutatividade dos operadores é o ponto de partida para a teoria de probabilidade quântica.

Na álgebra de Heisenberg, temos que $[P, Q]=-i \hbar I$. Como este comutador é proporcional à identidade, o valor esperado deste observável, $I$, para qualquer estado normalizado do sistema é 1. Assim, para as variáveis canônicas de posição e momento, o princípio da incerteza assume sua forma original, como proposta por Heisenberg:

$$
\sigma_{q} \cdot \sigma_{p} \geq \frac{\hbar}{2}
$$

O princípio da incerteza está vinculado intrinsecamente a duas questões que são fundamentalmente distintas. A primeira é esta que está descrita acima, a segunda, é o chamado microscópio de Heisenberg, vide (BRAGINSKY; KHALILI, 1995).

No caso descrito acima, temos à disposição idealmente infinitas cópias do sistema, todas elas, no mesmo estado. Ao realizarmos infinitas medições dos observáveis $A$ e $B$ teremos que a dispersão dos resultados será finita no limite da desigualdade (5.2). Este é um limite fundamental para a tecnologia.

No caso do microscópio de Heisenberg, temos apenas um sistema para ser analisado. Ao realizar um experimento de medição no sistema, cuidadosamente preparado, também nos deparamos com um limite fundamental dado explicitamente por

$$
\Delta x_{\text {medida }} \cdot \Delta p_{\text {perturbação }} \geq \frac{\hbar}{2} \text {. }
$$

A desigualdade (5.4) expressa que, fisicamente, ao realizarmos um experimento de medição de um observável do sistema, no caso acima a posição:

1 - A extração de informação do sistema a partir da medição do observável $x$ é associada a uma margem de erro finita;

2 - o experimento de medição causará uma perturbação na variável dinâmica $p$, também associada ao sistema;

3 - um processo irreversível ocorre inevitavelmente.

O ponto (3) acima decorre de uma análise de experimentos específicos e não se encontra diretamente expressa na desigualdade (5.4), a não ser pelo fato de que a perturbação em $p$ não poderá ser desfeita, pois, se pudesse, a composição das duas operações, medição e reconstituição da perturbação, resultaria em um experimento que violaria a desigualdade (5.4) . Experimentos de medição possuem uma vinculação inerente à desigualdade (5.4). Ambas, (5.4) e (5.3), são interdependentes, ou seja, são equivalentes fisicamente, vide Bragivski e Khalili (BRAGINSKY; KHALILI, 1995, pp. 8-10). Uma 
vez que o estado de um sistema quântico vincula os observáveis a um limite conjunto para suas respectivas variâncias, decorre que, em um experimento real de medição, a incerteza intrínseca a uma variável será propagada como perturbação para a variável canonicamente conjugada a ela. Em sentido oposto, a perturbação que inevitavelmente acompanha um experimento real de medição impede que um estado quântico seja preparado com valores precisos para um par de variáveis canônicas de tal forma que o produto dos respectivos desvios padrões violem a desigualdade (5.3).

Ressaltamos que mesmo em relação a uma álgebra QND, o elemento estocástico se encontra presente. O que ocorre é que em uma álgebra QND não existem experimentos incompatíveis entre si.

Os experimentos de observação podem ser vistos sob o ponto de vista da probabilidade, relacionando os resultados de uma medição quântica com uma variável aleatória. A não comutatividade dos operadores auto adjuntos que representam o experimento de medição implica a não comutatividade das variáveis aleatórias associadas. Esse fato é o ponto de partida para a generalização da teoria de probabilidade e do cálculo estocástico, para versões não comutativas compatíveis com os experimentos físicos na física quântica (SAKURAI, 1994; PARTHASARATHY, 1992; HUDSON; PARTHASARATHY, 1984; BOUTEN; HANDEL; JAMES, 2006b; BOUTEN; HANDEL; JAMES, 2006a).

\subsection{Medição e Dinâmica}

A incorporação dos efeitos provocados pelos processos de medição na dinâmica de sistemas quânticos é realizada por meio de modelos de sistemas quânticos abertos, que tornam possível a obtenção de modelos de evolução dissipativos a partir da dinâmica conservativa determinada pela equação de Schrödinger. Os efeitos não-conservativos na dinâmica quântica estão relacionadas a dois fenômenos importantes: a dissipação em sentido estrito, relacionada à troca de energia entre o sistema e seu meio, e a decoerência, relacionada aos efeitos do emaranhamento de sistemas quânticos. Os dois fenômenos estão relacionados entre si, mas a decoerência tem natureza essencialmente quântica.

A dinâmica de um experimento de medição, tomada como um processo contínuo no tempo, possui a mesma natureza física dos processos de decoerência. O sentido dessa afirmação, encontrada eventualmente na literatura é de que a medição está também relacionada ao emaranhamento de sistemas quânticos (BREUER; PETRUCCIONE, 2002; WISEMAN; MILBURN, 2010). Os postulados a respeito dos experimentos de medição em mecânica quântica apresentam em geral um modelo discreto, pelos quais a medição seria um processo instantâneo. Mostra-se que a dinâmica dos processos de medição contínuos, no limite em que o tempo de interação tende a zero e em que as condições de acoplamento entre o aparato de medição e o sistema são ideiais, apresenta os postulados 
da medição ortogonal de von Neumann-Lüders como limite.

A abordagem da dinâmica dos processos quânticos por meio da teoria de operações e efeitos leva a dois grandes resultados na teoria de sistemas dinâmicos abertos. O primeiro é a generalização do modelo matemático dos experimento de medição, com a teoria de medições POVM positive operator valued measure, e o segundo é a forma geral da equação de evolução que descreve a dinâmica não conservativa, a equação de Lindblad ou GoriniKossakowski-Sudarshan-Lindblad ${ }^{2}$. A forma mais conveniente de se representar o estado de um sistema quântico sujeito a uma dinâmica não conservativa é por meio de um operador densidade. A partir da teoria de operações e efeitos se pode buscar a forma geral das transformações no espaço dos operadores densidade. O teorema de representação de Kraus fornece a estrutura dessas transformações, que consistem em uma soma de composições de operadores que é linear no operador densidade e quadrática nos operadores que definem a transformação, que possuem forma livre.

A utilização da teoria generalizada de medições quânticas é capaz de conduzir a modelos matemáticos de processos de medições contínuos no tempo. Um dos modelos dinâmicos que podem ser utilizados é a equação de Schrödinger estocástica. Essa equação torna explícitos dois fatos importantes a respeito da dinâmica de sistemas quânticos que incorporam processos de medição contínuos:

1. A evolução infinitesimal do estado quântico no espaço de estados possui termos proporcionais ao operador que representa o observável e ao quadrado desse operador;

2. A dinâmica de evolução do estado é estocástica.

A evolução de um sistema dinâmico quântico sujeito a extração contínua de informação é, portanto, não linear. A forma dessa não linearidade, quadrática, é compatível com a forma geral das transformações de um estado quântico, dada pelo teorema de representação de Kraus. ${ }^{3}$ A forma do gerador infinitesimal da dinâmica da medição, contendo tanto termos proporcionais ao observável quanto termos proporcionais a seu quadrado, sugere também uma relação com o princípio da incerteza de Heisenberg.

Assim, a dinâmica de evolução de sistemas dinâmicos quânticos sujeitos a medição contínua está relacionada a uma álgebra dinâmica que contém tanto os operadores auto adjuntos que representam os observáveis quanto seus quadrados. Denominaremos essa

2 Tanto o artigo de Gorini-Kossakowski-Sudarshan quanto o artigo de Lindblad foram publicados em 1976. O de Lindblad aparentemente antes. No entanto, o de Gorini-Kossakowski-Sudarshan foi comunicado em data anterior ao periódico que o publicou. Também se encontram referências a preprints do trabalho do outro grupo em ambos. Assim, sem analisar o conteúdo e a qualidade dos artigos, seria injusto atribuir o mérito apenas a Lindblad. Entretanto, as referências da literatura à equação frequentementemente omitem o trabalho Gorini-Kossakowski-Sudarshan.

3 Deve-se observar que a equação de Schrödinger estocástica e as equações de evolução representadas pela soma de Kraus estão em formatos diferentes. Assim, chamamos a atenção para a correlação observada, no entanto, a relação entre esses fatos deve ser estabelecida mais formalmente. 
álgebra de álgebra de medição. Com o objetivo de determinar os efeitos de processos contínuos de medição na dinâmica dinâmica de uma sistema dinâmico quântico, é importante estabelecer a relação entre a álgebra de medição, a álgebra dinâmica e a álgebra de controle. Uma vez que a dinâmica do sistema é enriquecida de alguma forma pela álgebra de medição, é de se esperar que a álgebra de medição gere impacto nas propriedades de controlabilidade do sistema.

Considerando os efeitos causados pelo ruído induzido via medição, haverá certamente um limite para a intensidade dos processos de medição induzidos sobre o sistema, de forma a manter a incerteza causada pelos processos estocásticos dentro de um limite realístico. Os parâmetros de medição desempenham, com relação aos operadores de medição, um papel similar às funções de controle, com relação aos operadores da álgebra de controle.

Assim, podemos esperar, qualitativamente, que a álgebra de medição seja adicionada, de alguma forma, à álgebra de controle, gerando uma álgebra de controle quântico.

\subsection{Controlabilidade de Sistemas Quânticos}

Em dimensão finita os sistemas dinâmicos quânticos isentos de processos de medição e de interação com o meio ambiente, ou seja, na configuração de sistemas fechados, podem incorporar os resultados da teoria de controle clássica. Particularmente importantes são os resultados relacionados a sistemas bilineares expressos por meio da teoria de grupos e álgebras de Lie (D'ALESSANDRO, 2008; LISBOA, 2015; RAMAKRISHNA et al., 1995; ALBERTINI; D"ALESSANDRO, 2003).

Em dimensão infinita, mas ainda na configuração de sistemas fechados, os sistemas dinâmicos quânticos têm sua abordagem matemática dificultada. Surgem problemas relacionados à convergência no espaço de Hilbert e no espaço de transformações lineares que agem no espaço de Hilbert e problemas relacionados ao domínio de definição do sistema dinâmico quântico, causados pela presença de operadores não limitados associados à dinâmica do sistema. Essas questões foram abordadas na literatura em (HUANG; TARN, 1983; WU; TARN; LI, 2006). Seus resultados apontam que

1. Se a álgebra de controle tiver dimensão finita o sistema não poderá ser controlável no grupo de transformações unitárias de dimensão infinita que age no espaço de Hilbert;

2. Em dimensão infinita é possível ter sistemas de controle restritos a variedades integrais de dimensão finita, nessas condições, são aplicáveis os resultados e técnicas da teoria geométrica de controle (quando a álgebra de controle possui dimensão finita); 
3. A noção de controlabilidade em dimensão infinita deve ser aproximada para que dessa definição seja possível decorrer que a estratégia de controle seja finitamente executável, e, portanto, realista do ponto de vista das aplicações e da engenharia;

4. A solução para o domínio de definição do problema não é trivial, nem decorre da aplicação de técnicas convencionais da análise funcional, a não ser para uma classe restrita de problemas;

5. O conjunto dos vetores diferenciáveis parece ser uma ferramenta de aplicação geral na definição de sistemas dinâmicos quânticos em dimensão infinita, no entanto, sua abordagem é bastante complexa;

6. Quando a álgebra de controle possui dimensão infinita e satisfaz determinadas condições algébricas em relação à álgebra dinâmica, a variedade integral do sistema dinâmico associado no grupo de transformações unitárias do espaço de Hilbert possui dimensão infinita, no entanto, este resultado não estabelece as condições para que o conjunto de estados alcançáveis seja denso na esfera unitária do espaço de Hilbert;

7. A não-linearidade de algum elemento da álgebra de controle ou da álgebra dinâmica parece ser condição necessária à controlabilidade.

Na física matemática, em espaços de Hilbert de dimensão infinita, os vetores cíclicos estão relacionados a uma álgebra abeliana de operadores auto adjuntos que denominamos álgebra QND, vide (PRUGOVECKI, 1981). Em dimensão finita, os vetores cíclicos estão relacionados à controlabilidade de um sistema dinâmico linear pela forma canônica de Brunovski. Ressaltamos que a analogia é realizada entre duas classes de problemas diferentes, pois os sistemas dinâmicos quânticos de controle são bilineares. Pelos desenvolvimentos apresentados no Capítulo 3, não demonstramos que um sistema dinâmico, cuja álgebra de controle é uma álgebra QND é controlável no grupo de transformações gerado por essa álgebra QND, mas fazemos essa conjectura. Outro resultado importante, nessa mesma linha, é que a teoria de probabilidade quântica tem como caso especial a teoria de probabilidade clássica de Kolmogorov justamente os sistemas quânticos cujos observáveis são representados por operadores lineares auto adjuntos que compõem uma álgebra abeliana, ou seja, quando os operadores comutam e não há experimentos de medição incompatíveis entre si. Assim, o cálculo estocástico quântico se reduz ao cálculo estocástico clássico de Stratonovich-Itô e os sistemas quânticos cujas álgebras dinâmica e de medição estão restritas à mesma álgebra QND possuem equações dinâmicas similares às equações clássicas. ${ }^{4}$ Assim o comportamento das variáveis aleatórias associadas a ob-

4 A questão da equivalência entre as álgebras QND e a teoria de probabilidade clássica pode ser verificada em (BOUTEN; HANDEL; JAMES, 2006b; BOUTEN; HANDEL; JAMES, 2006a). As afirmações sobre o cálculo estocástico quântico devem ser verificadas com mais cuidado nas mesmas referências. 
serváveis em uma álgebra QND é equivalente ao comportamento de variáveis aleatórias clássicas. Essas conclusões são apresentadas em forma livre como conjecturas, devem ser investigadas com o devido cuidado e diligência.

Com relação à questão da não linearidade, que surgiu a partir da análise de exemplos apresentados em (WU; TARN; LI, 2006), podemos relacioná-la com vetores cíclicos, por meio da observação de que a definição de vetores cíclicos apresentados em (PRUGOVECKI, 1981) pressupõe a existência de uma álgebra de operadores composta de operadores não-lineares. Lembramos também que, em seus resultados, Prugovecki estabelece que um conjunto de operadores autoadjuntos é completo se, e somente se, possui um vetor cíclico. Da noção de completude apresentada também em Prugovecki decorre que a álgebra de operadores gerada é fechada para operações de soma e composição de operadores. Assim, qualquer polinômio gerado a partir dos operadores pode ser ser gerada dentro desta álgebra, vide, por exemplo, (REED; SIMON, 1980) ou (RIESZ; SZ.-NAGY, 1990). ${ }^{5}$

No tratamento de sistemas abertos em medição contínua, tanto os sistemas em dimensão finita quanto os sistemas em dimensão infinita apresentam efeitos dinâmicos determinados pelos operadores auto adjuntos que representam a medição. Como a dinâmica da medição apresenta termos não lineares, a incorporação de um processo de medição contínuo a um sistema de controle quântico em dimensão infinita poderia transformar uma álgebra de controle de dimensão finita em uma álgebra de controle de dimensão infinita.

$5 \quad$ Na realidade, os operadores auto adjuntos estão definidos para funções não apenas polinomiais, mas também funções contínuas ou apenas mensuráveis, vide (REED; SIMON, 1980, cap. VII) para uma discussão. 


\section{Conclusões e Sugestões de Trabalhos Fu- turos}

\subsection{Conclusões}

Para sistemas de controle quânticos fechados em dimensão finita, os métodos da teoria geométrica de controle possuem aplicação direta e imediata, devendo-se dar atenção apenas ao fato de os sistemas quânticos estarem definidos em espaços vetoriais complexos, vide (D'ALESSANDRO, 2008, pp. 160-161) para verificar um método de transformação para a conversão de sistemas dinâmicos em espaços vetoriais complexos para sistemas dinâmicos em espaços vetoriais reais.

A compreensão das teorias de probabilidade quânticas e do cálculo estocástico quântico é importante, do ponto de vista da engenharia, para que os modelos de sistemas quânticos com medição possam ser incorporados integralmente à teoria de sistemas e controle. Observamos que é possível a utilização de modelos baseados em cálculo estocástico clássico, permitindo um tratamento dos elementos estocásticos provenientes de processos de medição e de processos irreversíveis relacionados à interação com o meio ambiente. A incorporação do cálculo estocástico quântico aos métodos matemáticos na engenharia permitirá a modelagem do mesmo fenômeno a partir de um perspectiva diferente, o que muitas vezes é útil na solução de problemas práticos e teóricos.

A dinâmica não determinística de um sistema quântico que evolui em meio a processos dissipativos pode ser modelada por meio de três metodologias distintas, mas obviamente equivalentes: a equação de Schrödinger estocástica, SSE, a equação mestra estocástica,SME, e a equação diferencial estocástica quântica, QSDE, também conhecida como equação de Langevin quântica. Quando há, vinculado à dinâmica do sistema, um processo de medição, encontra-se associada uma equação que modela a extração de informação a respeito do estado do sistema. Tanta a equação que modela a dinâmica do estado quanto a equação que modela a extração de informação são estocásticas e são movidas, a cada instante de tempo, pelo mesmo ruído, ou seja, no caso da SSE e da SME, pelo mesmo incremento de Wiener. ${ }^{1}$

No regime quântico, há equações equivalentes à equação de Kushner-Stratonovich e às equações dinâmicas do filtro de Kalman-Bucy, assim, a partir de um processo de medição contínua acoplado a um sistema dinâmico quântico, é possível a obtenção de equações

1 No caso da QSDE, o elemento estocástico não é um incremento de Wiener (PARTHASARATHY, 1992; BOUTEN; HANDEL; JAMES, 2006b; BOUTEN; HANDEL; JAMES, 2006a; WISEMAN; MILBURN, 2010; JACOBS, 2014). 
que fornecem estimativas para o estado de sistemas dinâmicos. A primeira equação para um filtro quântico foi obtida na literatura por Belavkin e é amplamente utilizada em aplicações, principalmente na área da ótica quântica. Consideramos importante para a engenharia de sistemas quânticos o estudo dessa equação e sua capacterização. Um tópico de interesse é a determinação dos parâmetros físicos de maior impacto no projeto de sistemas reais. Outro tópico importante é a determinação do limite inferior para a geração de ruído em determinadas situaçoes de interesse. Ambos aspectos podem ser abordados de forma analítica ou de forma numérica. Um excelente paradigma, para a definição fatores de mérito para a comparação de performance entre sistemas abertos, que propomos simular, e sistemas fechados, pode ser encontrada em (LISBOA, 2015). A proposta de simulação de filtros na esfera de Bloch, apresentada abaixo, é baseada nessa necessidade.

$\mathrm{Na}$ análise de uma seleção de trabalhos científicos relacionados à controlabilidade de sistemas quânticos (HUANG; TARN, 1983; WU; TARN; LI, 2006; KARWOWSKI; VILELA-MENDES, 2004; VILELA-MENDES, 2009; VILELA-MENDES; MANIKO, 2011), notamos que as abordagens são realizadas de forma separada. Algumas são restritas ao grupo de transformações do espaço, enquanto outras, mais completas, estabelecem relações entre a álgebra de operadores auto adjuntos que compoe o sistema dinâmico e o grupo de transformações do espaço por elas gerado, (WU; TARN; LI, 2006), no entanto, deixam de abordar importantes aspectos topológicos do grupo de transformações. Para atingir controlabilidade aproximada em dimensão infinita é necessário que o sistema possua um conjunto denso de estados, que podem ser movimentados pela dinâmica do sistema de controle dentro de um subespaço também denso no espaço. Assim, de forma aproximada, posso partir de um estado arbitrariamente próximo de um estado inicial dado e chegar arbitrariamente próximo de um estado final de interesse. Utilizando um elemento encontrado em (KARWOWSKI; VILELA-MENDES, 2004), a noção de uma transformações de natureza infinita, exemplificada no artigo pela transformação de "shift", propomos uma variação. No lugar de apenas um operador infinito, posso partir de uma família de transformações simples, com um conjunto de propriedades que garanta que sua ação no espaço de estados não será restrita a um subspaço de dimensão finita. Na proposta de caracterização da controlabilidade apresentada abaixo sugerimos a utilização de uma família de transformações unitárias isomórfica a $S U(2)$. A partir de um teorema de decomposição do grupo $S U(n)$ em rotações planares, rotações de $S U(2)$, encontrado em (D'ALESSANDRO, 2008), e da família de transformações definida, acreditamos ter uma boa estrutura para abordar o problema de controlabilidade em $\ell^{2}$. É interessante acrescentar que os espaços de Hilbert de mesma dimensão são isomórficos, no entanto, a estrutura do espaço $\ell^{2}$ parece ser mais natural para abordar a dinâmica de computadores quânticos de dimensão infinita, utilizando a arquitetura de circuitos. 


\subsection{Sugestões de Trabalhos Futuros}

No Capítulo anterior, em que realizamos algumas discussões a respeito dos aspectos dinâmicos dos sistemas de controle quânticos, e em maior parte nos Capítulos 3 e 4, fizemos um conjunto de conjecturas a respeito da estrutura dos sistemas de controle quânticos. Acreditamos que investigações a respeito dessas conjecturas, possam levar a um algum avanço na compreensão desses sistemas. Destacamos a seguir algumas delas, sem prejuízo das outras:

1. investigação do papel desempenhado pelas álgebras QND, álgebras de operadores auto adjuntos que comutam, na dinâmica de sistemas quânticos em dimensão infinita e a relação que têm com sistemas de controle clássicos determinísticos e estocásticos;

2. investigação a respeito da natureza do conjunto de operadores associados aos experimentos de medição e de sua relação com a álgebra de controle do sistema dinâmico quântico;

3. investigação sobre o limite inferior da quantidade de ruído associada aos processos de medição e da relação entre esse limite e as álgebras dinâmica, de controle e de medição; ${ }^{2}$

4. investigação sobre os efeitos da não linearidade dos processos de medição na controlabilidade de sistemas quânticos em dimensão infinita;

5. relação entre a não linearidade em sentido fraco e a controlabilidade de sistemas quânticos QND e sobre sistemas quânticos gerais.

\subsubsection{Caracterização da Controlabilidade em Dimensão Infinita}

Apresentamos a seguir um conjunto de proposições que tem por objetivo propor um método de caracterização da controlabilidade em dimensão infinita. É um esboço, por isso se encontra neste capítulo como uma sugestão para trabalho a ser desenvolvido futuramente. Estas proposições tiveram como ponto de partida os trabalhos de VilelaMendes, (KARWOWSKI; VILELA-MENDES, 2004; VILELA-MENDES, 2009; VILELAMENDES; MANIKO, 2011), que apontaram a necessidade de um grupo de transformação capaz de agir de maneira não trivial em qualquer elemento da base canônica de $\ell^{2}$. A aplicação por esses autores destacada para desempenhar este papel foi a aplicação de "shift", cuja eficiência fora demonstrada a partir de um exemplo fictício.

2 Uma vez que esta taxa de geração de ruído está associada ao princípio da incerteza, o conjunto ótimo de operadores auto adjuntos que agem no sistema dinâmico no mesmo instante, $\bar{t}$, deve ser escolhido de forma a minimizar a relação de incerteza para o estado quântico $\rho(\bar{t})$. Esse raciocínio vale para uma função de custo puramente associada associada à taxa de geração de ruído. 
Substituímos a aplicação de "shift" por um conjunto de transformações unitárias bidimensionais capaz de agir em qualquer subespaço bidimensional de $\ell^{2}$ e procuramos encadear uma demonstração de que qualquer $x \in \ell^{2}$ pode ser levado de forma aproximada a qualquer outro $y \in \ell^{2}$. Enunciamos também a proposição para o caso exato, no entanto, sua demonstração irá exigir muito cuidado no tratamento da convergência de operadores no espaço dos operadores lineares de $\ell^{2}$. Acreditamos também que a proposição para o caso exato não será necessária para expressar um conceito de controlabilidade aproximada em dimensão infinita.

Alcançamos assim, obviamente na forma de conjectura, o resultado de que posso aproximar qualquer $y \in \ell^{2}$ a partir de qualquer outro elemento inicial $x_{0} \in \ell^{2}$, tomando uma composição finita de elementos do grupo de transformações unitárias bidimensionais definido. Assim, um sistema dinâmico caracterizado por um grupo de transformações desse tipo é controlável de forma aproximada. Utilizamos então o teorema de Stone para transpor este resultado à álgebra de operadores, buscando a classe de equivalência do conjunto de operadores auto-adjuntos capaz de gerar um conjunto de transformações unitárias bidimensionais "completo" em $\ell^{2}$.

Ao final, provamos que um sistema de controle será controlável em $\ell^{2}$ se, e somente se, sua álgebra de controle for capaz de gerar um conjunto de transformações unitárias bidimensionais como o definido. Assim, como a transformação de "shift" e o conjunto de transformações unitárias definido acima, outras transformações ou conjuntos de transformações podem ser utilizadas para caracterizar os sistemas dinâmicos controláveis.

Acreditamos que a proposta do grupo de transformações aqui realizada tem a vantagem der um conjunto de elementos simples, para o qual é feita uma transposição para a álgebra de operadores auto-adjuntos, levando a um conjunto de elementos possivelmente simples. ${ }^{3}$

A partir do conjunto de operadores auto-adjuntos definido, ou dos elementos de sua classe de equivalência, a análise de controlabilidade pode ser realizada na álgebra de controle, ou nos campos de vetores em linguagem equivalente. Poderemos então buscar, por exemplo, decomposições dos operadores auto-adjuntos em subespaços invariantes de tal modo que as restrições dos operadores aos subespaços invariantes seja equivalente ao conjunto "completo" de operadores auto-adjuntos obtido pelo teorema de Stone, a partir do conjunto de transformações unitárias definido.

Apresentamos também duas definições que não encontramos na literatura. Uma referente a sistemas de energia confinada, que se refere a sistemas cuja dinâmica não

3 Cada elemento do conjunto deve possuir uma classe de equivalência que é dada por operadores que possuem um domínio denso em $\ell^{2}$ e que coincidem, nesse domínio, com uma aplicação auto-adjunta que seja definida em todo o espaço. Assim, no caso de operadores não-limitados, mesmo que a álgebra de controle seja capaz de gerar um conjunto transitivo de transformações lineares, o domínio comum das transformações auto-adjuntas associadas pode não ser denso no espaço. 
excita todos os seus graus de liberdade e outra referente a sistemas de controle quântico com famílias de Hamiltonianos de controle indexadas por parâmetro contínuo.

Vamos então à proposta. Alertamos, no entanto, que as demonstrações, quando oferecidas, são apenas esboços que foram utilizados tanto para ajudar na composição do raciocínio desenvolvido e quanto para realizar uma análise de viabilidade mínima da proposta. Para dar mais embasamento a essa proposta citamos um resultado sobre decomposição de transformações unitárias encontrado em (D'ALESSANDRO, 2008, pp. 130-131).

Seja $\left\{U_{i j}\right\}$ um conjunto de transformações unitárias tal que cada elemento $U_{i j}$ age no subespaço gerado por $\left\{e_{i}, e_{j}\right\}$ em $\ell^{2}$, denotado por $\left\{e_{i}, e_{j}\right\}_{V S}$.

Proposição 59. Se $\left\{X \in \ell^{2} \mid\|X\|=1\right\}$ e $X \in\left\{e_{i}, e_{j}\right\}_{V S}$ então existe uma composição de $\left\{U_{\alpha \beta}\right\}$ que transporta $X$ para $\left\{e_{\alpha_{1}}, e_{\alpha_{1}}\right\}_{V S}$ quaisquer que sejam $e_{\alpha_{1}}, e_{\alpha_{2}}$ elementos da base canônica.

Demonstração. $\exists \theta_{1}$ e $\varphi_{1}$ tais que $U_{i j}\left(\theta_{1}, \varphi_{1}\right) X=e_{i}$ ou $e_{j}$.

Tomo $U_{i \alpha_{1}}$ e escolho $\theta_{2}$ e $\varphi_{2} \Rightarrow U_{i \alpha_{1}}\left(\theta_{1}, \varphi_{1}\right) e_{1}=e_{\alpha_{1}}$.

Posso escolher $\theta_{3}$ e $\varphi_{3}$ de tal forma que $U_{\alpha_{1} \alpha_{2}}\left(\theta_{3}, \varphi_{3}\right) e_{\alpha_{1}}=y$, qualquer que seja $y \in\left\{e_{\alpha_{1}}, e_{\alpha_{2}}\right\},\|y\|=1$.

Proposição 60. Seja $X \in \ell^{2},\|X\|=1$ então existe em $n \in \mathbb{N}$ uma composição finita de $\left\{U_{i j}\right\}$ tal que $\left\langle U_{\alpha_{n} \beta_{n}} \ldots \alpha_{1} \beta_{1} X, e_{i}\right\rangle \geq 1-\epsilon$ qualquer que $\epsilon>0$ escolhido.

Demonstração. Seja $\left(X_{i}\right)_{I}$ a representação de X na base canônica de $\ell^{2}$.

Posso permutar os elementos da base canônica para ordenar as projeções $X_{i}$ de forma decrescente.

Terei então $\left(X_{i}\right)_{\pi}$, a representação de $\mathrm{X}$ na base permutada.

Seja $\left\{U_{i j}\right\}_{\pi}$ o conjunto de TU devidamente permutado

$\left(x_{0}, x_{1}\right)_{\pi}$ é a projeção de $\mathrm{X}$ em $\left\{e_{0}, e_{1}\right\}_{\pi(V S)}$

Tomo $U_{01}$ em $\left\{U_{i j}\right\}_{\pi}$ e escolho $\theta_{1}$ e $\varphi_{1}$ tais que $\left\langle U_{01}\left(\theta_{1}, \varphi_{1}\right) X, e_{1}\right\rangle=0$ ou seja, rotaciono $\mathrm{X}$ no subespaço $\left\{e_{0}, e_{1}\right\}$ de tal forma que $\left(X_{1}\right)_{\pi}=0$ 
Em seguida, tomo $U_{02}$ e escolho $\theta_{2}$ e $\varphi_{2}$ tais que $\left\langle U_{02}\left(\theta_{2}, \varphi_{2}\right) U_{01}\left(\theta_{1}, \varphi_{1}\right) X, e_{2}\right\rangle=0$

$\mathrm{Na}$ realidade, posso fazer o produto escalar com qualquer $\left(a_{1} e_{1}+a_{2} e_{2}\right)$ uma vez que $U_{02}$ age como identidade fora do subespaço $\left\{e_{0}, e_{1}\right\}_{V S}$

Faço isso até que $\sum_{i>n}\left|x_{i}\right|^{2}<\epsilon$

Fico com $U_{0 n}\left(\theta_{n}, \varphi_{n}\right) \cdots U_{01}\left(\theta_{1}, \varphi_{1}\right) X$

Faço a permutação inversa e respeitando as equivalências em $\left\{U_{i j}\right\}$ e $\left\{U_{i j}\right\}_{\pi} \sim U_{01}$

Seja $e_{i}=\pi^{-1}\left(e_{0}\right)$

Como $\sum_{i>n}\left|x_{i}\right|^{2}<\epsilon$ é invariante à permutaçã, assim como $\theta_{i}, \varphi_{i}$,

$\left\langle U_{\alpha_{n} \beta_{n}} \ldots \alpha_{1} \beta_{1} X, e_{i}\right\rangle \geq 1-\epsilon$

Proposição 61. Seja $X \in \ell^{2},\|X\|=1$ então existe uma composição finita de $\left\{U_{i j}\right\}$, possivelmente com infinitos termis, tal que

$$
\underbrace{U=\left[\cdots U_{\alpha_{n} \beta_{n}} \cdots U_{\alpha_{1} \beta_{1}}\right]}_{\text {essa convergência não é trivial! }} \Rightarrow U x=e_{i}, \forall \text { algum } e_{i} \text { da base canônica. }
$$

Proposição 62. Seja $y \in \ell^{2},\|y\|=1$ e $\left\{U_{i j}\right\}$ um conjunto de transformações unitárias que age em $\left\{e_{i}, e_{j}\right\}_{V S}$ como $U(2)$ e como identidade no restante do espaço, então

$\exists U \in\left\{U_{i j}\right\}_{G L}$ tal que $U e_{0}=y$

Demonstração. Toma a $U$ da proposição anterior e verifico $e_{i}$

Se $e_{i}=e_{0}$, tomo $U=\bar{U}^{-1}$

Se $e_{i} \neq e_{0}$, toma $U_{i 0}$ em $\left\{U_{i j}\right\}_{G L}$ e escolho $\theta_{1}, \varphi_{1}$ tais que $U_{i 0}\left(\theta_{1}, \varphi_{1}\right) e_{0}=e_{i}$

Faço então $U=\bar{U}^{-1} U_{i 0}\left(\theta_{1}, \varphi_{1}\right)$

Proposição 63. Seja $y$ e $\left\{U_{i j}\right\}_{G L}$ então

$\exists U \in\left\{U_{i j}\right\}_{G L}$ tal que

$\left\|U e_{0}-y\right\|^{2}=\left\langle U e_{0}-y, U e_{0}-y\right\rangle<\epsilon, \forall \epsilon>0$ dado 
Proposição 64. Sejam $x, y \in \ell^{2},\|x\|=\|y\|=1$ e $\left\{U_{i j}\right\}_{G L}$ então existe $U \in\left\{U_{i j}\right\}_{G L}$ tal que

$$
U x=y
$$

Demonstração. Aplicação de duas proposições anteriores.

Proposição 65. Sejam $x, y \in \ell^{2},\|x\|=\|y\|=1$ e $\left\{U_{i j}\right\}_{G L}$ então existe $U \in\left\{U_{i j}\right\}_{G L}$ tal que

$$
\|U x-y\|^{2}\langle U x-y, U x-y\rangle<\varepsilon, \forall \varepsilon>0, \text { dado }
$$

Proposição 66. Composições finitas de $\left\{U_{i j}\right\}$ são aproximadamente transitivas em $\mathcal{U}\left(\ell^{2}\right)$, o grupo das transformações unitárias de $\ell^{2}$, ou seja,

dada $U \in \mathcal{U}\left(\ell^{2}\right), \exists U_{n}$, composição finita de $\left\{U_{i j}\right\}$ ou $U_{n} \in\left\{U_{i j}\right\}^{n}$

$$
\left\|U-U_{n}\right\|^{2}<\epsilon, \forall \epsilon>0 \text { dado }
$$

Demonstração. Escolho $x \in \ell^{2}$ e calculo $y=U x$

Aplico a proposição anterior até que $\left\|\bigcup_{n} x-y\right\|^{2}<\delta$

Escolho os espaços e a norma em $L\left(\ell^{2}, \ell^{2}\right)$ tal que a convergência em $\ell^{2}$, relacionada a $\delta$ implique a convergência em $\mathcal{U}\left(\ell^{2}\right)$, relacionada a $\epsilon$

Proposição 67. Sejam $\left\{A_{i j}\right\}$ o conjunto de operadores auto-adjuntos agindo em $\ell^{2}$, associado ao conjunto $\left\{U_{i j}\right\}$ pelo teorema de Stone.

Se um sistema de controle quântico possui uma álgebra que contém $\left\{A_{i j}\right\}$, então sua dinâmica é controlável em $\ell^{2}$.

De forma exata, utilizando uma sequência possivelmente infinita de pulsos $\left\{u_{\alpha} A_{i j}\right\}$.

De forma aproximada, utilizando uma sequência finita de pulsos de controle. 
Proposição 68. Se o sistema de controle possui uma álgebra capaz de aproximar as $\left\{A_{i j}\right\}$ de tal forma que

$$
A n \rightarrow A_{i j} \Rightarrow e^{i A_{n}}=U_{A_{n}} \rightarrow U_{i j}
$$

Então sua dinâmica é aproximadamente controlável em $\ell^{2}$

A proposição acima só é diferente da anterior se a álgebra de controle não for fechada (localmente compacta).

Proposição 69. Um grupo $\left\{V_{\alpha}\right\}_{G L}$ é transitivo, ou aproximadamente transitivo, em $\mathcal{U}\left(\ell^{2}\right)$ se, e somente se,

$$
\left\{V_{\alpha}\right\}_{G L} \text { contém }\left\{U_{i j}\right\}
$$

Demonstração. Se $\left\{V_{\alpha}\right\}_{G L}$ é transitivo $\Rightarrow \forall U_{i j}, \exists \bar{\alpha}$ tal que $V_{\bar{\alpha}}=U_{i j}$, portanto, $\left\{V_{\alpha}\right\}$ contem $\left\{U_{i j}\right\}$.

Agora, se $\left\{V_{\alpha}\right\} \geq U_{i j}$, pela aplicação das proposições anteriores $\left\{V_{\alpha}\right\}$ é transitiva.

Em princípio, podem existir várias famílias $\left\{V_{\alpha}\right\}$ diferentes entre si e transitivas em $\mathcal{U}\left(\ell^{2}\right)$, mas elas são equivalentes entre si no sentido da proposição anterior. Aliás, nem é necessário que, na hipótese da proposição, seja tomada $\left\{V_{\alpha}\right\}_{L A}$. É suficiente considerar $\left\{V_{\alpha}\right\}$.

Definição 70 (Sistemas com energia confinada). Seja $S=\left\{e_{0}, \cdots, e_{n}\right\}_{V S}$ o suporte de um sistema dinâmico quântico de tal forma que todo estado $X$ pertecente a um domínio de interesse $D$ seja tal que

$\left\{X \in D:\left\|\left(I-P_{S}\right)_{X}\right\|<\epsilon\right\}$ onde $P_{S}$ é a projeção em $S$, ou seja, a norma de $X$ fora de $S$ é desprezível.

Então $U(n)$ é uma boa álgebra de controle para o sistema, pois o erro cometido pela aproximação é limitado.

Fisicamente posso dizer que a energia do sistema está confinada ao subespaço suporte.

A propriedade cíclica do traço não é válida em dimensão infinita, assim como a forma de Lindblad. 
Há evidência de que estas estruturas são válidas, pelo menos de forma aproximada. Na literatura física é constantemente utilizada em dimensão infinita, no entanto, não há evidência experimental que os modelos matemáticos que utilizam essas propriedades e estruturas estejam incorretos. Como o estado de um sistema quântico é um elemento de um espaço de Hilbert, não é possível que sua decomposição em uma base ortonormal não contenha o zero como ponto de acumulação. Assim, todo sistema física pode ser visto como um sistema de energia confinada. Como apresentamos ao longo do trabalho, os físicos frequentemente justificam essas manipulações pela impossibilidade física de haver um sistema com energia infinita. Esta definição é a proposta de um ponto de partida para a formalização dessa ideia.

Definição 71 (Famílias de controle a parâmetro contínuo). A questão da controlabilidade finita pode também ser abordada para um sistema dinâmico do tipo

$$
\psi=\left[H_{0}+u(t) A_{u}\right] \psi
$$

onde $A_{u}$ é uma família de operadores auto-adjuntos parametrizada em $u \in[a, b] \subseteq$ $\mathbb{R}$

$\left\{A_{u}\right\}_{u \in[a, b]}$ pode compor a álgebra $\left\{A_{u}: u \in[a, b]\right\}_{L A}$

A possibilidade de utilizar famílias de operadores lineares a parâmetro contínuo pode levar a um grupo de transformações unitárias denso em $\mathcal{U}\left(\ell^{2}\right)$ que seja composto por $U\left(u_{1}, u_{2}, \cdots, u_{n}\right) \operatorname{com}\left(u_{1}, u_{2}, \cdots, u_{n}\right) \in \mathbb{R}^{n}$

$$
\dot{\psi}=\left[H_{0}+\sum u_{i} A_{u_{i}}\right] \psi
$$

Na realidade $U$ dependerá também de $\left(u_{1}, u_{2}, \cdots, u_{n}\right) \in \mathbb{R}^{n}$

Um questão importante é a equivalência entre a álgebra gerada por uma família $A_{\gamma}$ e uma álgebra $\left\{A_{i}\right\}_{L A}$ finitamente ou infinitamente gerada.

\subsubsection{Simulação de Filtros na esfera de Bloch}

Definição 72 (Medições dinâmicas). Seja $\mathcal{H}^{2}$ um espaço de Hilbert bidimensional e $\hat{n}=n(\theta, \rho)$ um vetor unitário que define uma direção arbitrária em $\mathbb{R}^{3}$. Seja $S_{\hat{n}}$ o operador associado ao observável momento angular na direção $\hat{n}$, vide Sakurai para uma construção desse operador, que possui autovetores $|\hat{n}+\rangle$ e $|\hat{n}-\rangle$. 
Agora consideremos que seja possível alterar dinamicamente a direção característica do operador, obtendo assim um $S_{\hat{n}}(t)$, que ainda é um operador na perspectiva de Schrödinger, apesar da dependência temporal.

Sejam $\vec{u}$ e $\vec{v}$ dois vetores ortogonais em $\mathbb{R}^{3}$ tal que $\vec{u} \times \vec{v}=\hat{n}$, onde consideramos que $\|\vec{u}\|=\|\vec{v}\|=1$, e sejam $S_{u}$ e $S_{v}$ os operadores associados ao momento angular nas direções $u$ e $v$. As direções $u$ e $v$ também podem ser vistas como um par de direções ortogonais em uma carta local na esfera unitária em $\mathbb{R}^{3}$ em torno do ponto definido pelo vetor $\hat{n}$, tomando a esfera como uma variedade diferenciável. Façamos agora a identificação da esfera unitária à esfera de Bloch.

Seja $\bar{\rho}(t)$ o operador densidade de um sistema dinâmico e $\rho(t)$ a melhor estimativa para seu valor. Considerando que o erro na estimativa é baixo, posso realizar medições contínuas nas direções ortogonais no plano definido por $\rho(t)$ em $\mathbb{R}^{3}$ e corrigir o curso de $\rho(t)$ na esfera de Bloch para que seja, dentro das margens de erro possíveis uma trajetória especificada. Para controlar a posição de $\rho(t)$ utilizo também os operadores $S_{u}$ e $S_{v}$.

Os operadores $S_{\hat{n}}, S_{u}$ e $S_{v}$ definem um sistema de medição e controle na esfera de Bloch capaz de agir de forma equivalente em qualquer direção definida por um operador densidade, de tal forma que o sistema dinâmico não apresenta singularidades na superfície da esfera de Bloch.

Um sistema de medição assim definido pode ser usado como um paradigma teórico para a comparação de outros sistemas dinâmicos, isso porque utiliza dois operadores de medição e de controle. Esta configuração utilizada em tese minimiza o esforço de controle pois $S_{u}$ e $S_{v}$ são os geradores de rotação em duas direções ortogonais a $\hat{n}$. Assim, o sistema proposto é construído de forma que apresente baixo ruído e baixo esforço de controle.

Uma sugestão para trabalhos futuros é caracterizar o sistema de medição e controle definido por $S_{\hat{n}}, S_{u}$ e $S_{v}$ podendo

1 - Caracterizar o sistema estático associado, $H_{0}=0$, com estado inicial real $|\psi\rangle=|z+\rangle$, valor esperado pelo observador $\rho=|\psi\rangle\langle\psi|$ e $S_{\hat{n}}=S_{z}, S_{u}=S_{x}$ e $S_{v}=S_{y}$, verificando o estado real do sistema e o erro definido pela diferença entre o estado real e a melhor estimativa para o estado. Utilizar vários valores para a intensidade de medição $\kappa$, obter o erro do sistema na condição de regime para cada valor de $\kappa, \delta_{\kappa}$, assim como a norma do operador densidade em regime, $\|\rho\|$, ou $\left\|\rho^{2}\right\|$, que dá uma medida de quão próxima a estimativa se encontra de um estado puro.

2 - Caracterizar o mesmo sistema estático acima utilizando intensidades de medição dinâmicas, partindo de um estado de incerteza total, buscando atingir a incerteza mínima de regime no menor tempo possível. Define-se assim um problema de medição ótimo. Verificar se existe algum tipo de dualidade entre controle ótimo e medição ótima, assim como existe dualidade entre controlabilidade e observabilidade em sistemas clássi- 
cos. Uma tal dualidade permitiria a transposição dos métodos de programação dinâmica e do princípio do máximo de Pontryagin para o problema de medição ótima.

3 - Caracterizar o sistema de medição dinâmica associado para o caso em que o Hamiltoniano é conhecido, utilizando as mesmas variações propostas em 1 e 2 acima. Verificar em particular se existe uma resposta em frequência para o erro, ou seja, um aumento no valor do erro de regime para frequências características $\omega$ presentes no Hamiltoniano. Utilizar os estimadores usuais para $\langle x\rangle,\langle y\rangle,\langle z\rangle$ as coordenadas de $\rho$ na esfera de Bloch. Verificar igualmente se a frequência afeta $\left\|\rho^{2}\right\|$ e o valor de regime de $\kappa(t)$.

4 - Caracterizar o sistema de medição definido para o caso dinâmico em que o Hamiltoniano é parcialmente ou totalmente desconhecido, investigando assim um problema de identificação de sistemas dinâmicos quânticos.

Nas simulações, utilizar o sistema

$$
\begin{aligned}
d \rho & =-i[H, \rho]+\sum 2 \kappa_{i} \mathcal{D}\left[S_{i}\right] \rho+\sum \sqrt{2 \kappa_{i}} \mathcal{H}\left[S_{i}\right] \rho d W_{i}, \quad i \in\{u, v\} \\
d x_{i} & =\left\langle S_{i}\right\rangle d t+\frac{1}{\sqrt{8 \kappa_{i}}} d W_{i} \\
H & =H_{0}+\sum_{i} u_{i} S_{i} .
\end{aligned}
$$

Os resultados obtidos podem ser usados para comparação com o caso de medição estática que podem ser simulados pelo sistema estocástico:

$$
d \rho=-i[H, \rho] d t+\sum_{i} 2 \kappa_{i} \mathcal{D}\left[S_{i}\right] \rho d t+\sum_{i} \sqrt{2 \kappa_{i}} \mathcal{H}\left[S_{i}\right] \rho d W_{i}
$$

onde $i \in\{x, y, z\}, S_{i}$ são as matrizes de Pauli, $\kappa_{i}=\kappa_{i}(t)$ são as intensidades de medição e $d W_{i}$ são incrementos de Wiener independentes.

A equação (6.4) está condicionada a

$$
d x_{i}=\left\langle S_{i}\right\rangle d t+\frac{1}{\sqrt{8 \kappa_{i}}} d W_{i}
$$

O Hamiltoniano $H$ é da forma $H=H_{0}+u_{i} S_{i}$.

Uma vez que $S_{\hat{n}}=\vec{S} \cdot \vec{n}$, vide Sakurai, o sistema (6.4), (6.5) acima não é em geral equivalente ao sistema (6.1), (6.2) acima pois os operadores $\mathcal{D}$ e $\mathcal{H}$ são não-lineares. No entanto, o sistema (6.4), (6.5) é geometricamente simples e também pode ser usado como forma de familiarização com as equações de filtragem e medição contínuas. Deve-se notar que o sistema acima possui redundâncias, assim, em geral, posso ter situaçõoes em que algum $\kappa_{i}$ e algum $u_{i}$ sejam irrelevantes.

É importante também saber o impacto dos superoperadores $\mathcal{D}$ e $\mathcal{H}$ nas diferentes performances encontradas na dinâmica dos sistemas de medição estático e dinâmico. Como o sistema estático tratalha com operadores adicionais para realizar medição e controle, essas diferenças são esperadas. 


\subsubsection{Medição quântica e classificação dinâmica}

De acordo com a definição dada para um POVM a descrição dada ao processo de medição é realizada a partir de um conjunto de operadores $\Omega_{m \kappa}$, que define uma família de superoperadores $\Phi_{m}$ que agem em um operador densidade como um sistema dinâmico discreto, levando $\rho_{0} \mapsto \Phi\left(\rho_{0}\right)$. A estrutura matemática de $\Phi$ é dada pelo teorema de representação de Kraus

$$
\Phi(\rho)=\sum_{\kappa} \Omega_{\kappa} \rho \Omega_{\kappa}^{\dagger}
$$

A proposta de trabalho que se faz é utilizar a teoria de sistemas dinâmicos e aplicála e $\Phi$, que é uma transformação que gera uma dinâmica discreta no espaço de estados. Os resultados podem ser utilizados para caracterizar os experimentos de medição a partir da dinâmica que causam no espaço de estados.

Inicialmente podem ser investigados e caracterizados as medições realizadas na esfera de Bloch.

A análise dos sistemas dinâmicos deve ser feita a partir da restrição de que $\sum F_{m}=$ $\sum \Omega_{m}^{\dagger} \Omega_{m}=I$, o que garante que a família de sistemas dinâmicos analisada é um POVM.

Dessa forma, a consolidada e abrangente teoria de sistemas dinâmicos pode ser aplicada à teoria generalizada de medição quântica com possíveis resultados úteis. Lembremos também que, ao deduzir as equações estocásticas que descrevem a medição contínua, encontramos termos não-lineares. Assim, é possivel que encontremos famílias de sistemas dinâmicos associadas a POVM's de tal forma que os sistemas dinâmicos sejam caóticos.

Devemos lembrar, contudo, que a terminologia não-linear em sistemas quânticos está associada a sistemas quânticos que possuem equivalente clássico não-linear. Lembremos também que os superoperadores são por definição mapas lineares em $\rho$, vide (PRESKILL, 1997). No entando, observando a estrutura da soma no teorema de representação de Kraus (6.6), podemos encontrar consistência com a equação de Schrödinger estocástica apresentada no capítulo 4 (4.67), que apresentava termos não-lineares quadráticos associados a um processo de medição. Isso porque os operadores de medição do POVM aparecem de forma quadrádica na soma de Kraus.

Inversamente, também seria interessante, do ponto de vista de sistemas dinâmicos, associar a um sistema não-linear definido em um espaço de dimensão finita um sistema linear definido em um espaço de Hilbert. 


\section{Considerações finais}

Realizamos um panorama da teoria de controle de sistemas quânticos a partir dos resultados existentes na literatura. Consideramos os casos de sistemas quânticos em dimensão finita e infinita submetidos ou não a processos de medição. Foram feitas analogias com sistemas clássicos lineares e buscou-se analisar a dinâmica dos sistemas quânticos de controle considerando os efeitos provocados pelos processos de medição, em particular nas propriedades de controlabilidade. Apresentamos, ao final, um conjunto de tópicos a serem investigados, com o objetivo de dar continuidade ao trabalho realizado. 


\section{Referências}

AGRACHEV; SACHKOV. Control Theory from the geometric viewpoint. [S.l.]: Springer, 2004. Citado 2 vezes nas páginas 49 e 51.

AGRAWAL; KAYAL; SAXENA. Primes is in p. Annals of Mathematics, v. 160, p. 781-793, 2004. Disponível em: <http://www.jstor.org/stable/3597229>. Citado 3 vezes nas páginas 14, 39 e 44.

ALBERTINI, F.; D"ALESSANDRO, D. Notions of controllability for bilinear multilevel quantum systems. Automatic Control, IEEE Transactions on, v. 48, n. 8, p. 1399-1403, 2003. Citado 2 vezes nas páginas 50 e 152.

ANOSOV et al. Dynamical Systems I: Ordinary Differential Equations and Smooth Dynamical Systems. [S.1.]: Springer, 1988. Citado 3 vezes nas páginas 20, 21 e 206.

ARNAL, D. Symmetric nonself-adjoint operators in an enveloping algebra. Journal of Functional Analysis, v. 21, n. 432, 1976. Disponível em: <http: //dx.doi.org/10.1016/0022-1236(76)90036-7>. Citado na página 77.

ARNOLD. Ordinary Differential Equations. [S.1.]: MIT Press, 1978. Citado 2 vezes nas páginas 20 e 206 .

ARNOLD, V. I. Mathematical Methods of Classical Mechanics. [S.l.]: Springer, 1997. Citado na página 20.

BARNES, E.; SARMA, S. D. Analytically solvable driven time-dependent two-level quantum systems. Phys. Rev. Lett., American Physical Society, v. 109, p. 060401, Aug 2012. Disponível em: < doi:10.1103/PhysRevLett.109.060401>. Citado na página 17.

BASDEVANT, J.; DALIBARD, J. Quantum Mechanics. [S.l.]: Springer, 2005. Citado na página 27.

BELAVKIN, V. P. Quantum continual measurements and a posteriori collapse on ccr. Communications in Mathematical Physics, v. 146, n. 3, p. 611-635, 1983. Disponível em: <http://dx.doi.org/10.1007/BF02097018>. Citado na página 54.

BELAVKIN, V. P. Theory of control of observable quantum systems. Automatica and Remote Control, v. 44, p. 178-188, 1983. Citado na página 54.

BELAVKIN, V. P. Non-demolition measurement and control in quantum dynamical systems. In: . Information complexity and control in quantum physics. Udine: Springer, 1985. p. 311-330. Citado na página 54.

BENIOFF, P. Quantum mechanical models of turing machines that dissipate no energy. Phys. Rev. Lett., American Physical Society, v. 48, p. 1581-1585, Jun 1982. Disponível em: < doi:10.1103/PhysRevLett.48.1581>. Citado na página 43.

BENIOFF, P. Unitary dilation models of turing machines in quantum mechanics. Phys. Rev. A, American Physical Society, v. 51, p. 3513-3524, May 1995. Disponível em: $<$ doi:10.1103/PhysRevA.51.3513>. Citado na página 42. 
BENIOFF, P. Tight binding hamiltonians and quantum turing machines. Phys. Rev. Lett., American Physical Society, v. 78, p. 590-593, Jan 1997. Disponível em: $<$ doi:10.1103/PhysRevLett.78.590>. Citado na página 43.

BENIOFF, P. Models of quantum turing machines. Fortsch. Phys., v. 46, p. 423-442, 1998. Citado na página 43.

BERKOVITZ, J. Action at a distance in quantum mechanics. In: ZALTA, E. N. (Ed.). The Stanford Encyclopedia of Philosophy. Spring 2014. [s.n.], 2014. Disponível em: $<$ http://plato.stanford.edu/archives/spr2014/entries/qm-action-distance/>. Citado na página 57.

BIRKHOFF; NEUMANN von. The logic of quantum mechanics. Apr 1936. Disponível em: <http://www.jstor.org/stable/1968621>. Citado 2 vezes nas páginas 42 e 57.

BLAIS, A. et al. Cavity quantum electrodynamics for superconducting electrical circuits: an architecture for quantum computation. Phys. Rev. A, American Physical Society, v. 69, p. 062320, Jun 2004. Disponível em: < doi:10.1103/PhysRevA.69.062320>. Citado 4 vezes nas páginas 46, 47, 55 e 56 .

BLAIS, A. et al. Quantum-information processing with circuit quantum electrodynamics. Phys. Rev. A, American Physical Society, v. 75, p. 032329, Mar 2007. Disponível em: $<$ doi:10.1103/PhysRevA.75.032329>. Citado na página 47.

BLOCH, A. M.; BROCKETT, R. W.; RANGAN, C. The controllability of infinite quantum systems and closed subspace criteria. n. quant-ph/0608075, Aug 2006. Citado na página 53.

BOHM, A. Quantum Mechanics: Foundations and applications. [S.l.]: Springer, 1994. Citado 2 vezes nas páginas 27 e 80.

BOUTEN; HANDEL, v.; JAMES. A discrete invitation to quantum filtering and feedback control. Jun 2006. Disponível em: < arXiv:math/0606118v4>. Citado 7 vezes nas páginas 54, 55, 127, 129, 150, 153 e 155.

BOUTEN; HANDEL, v.; JAMES. An introduction to quantum filtering. Jan 2006. Disponível em: <arXiv:math/0601741v1>. Citado 9 vezes nas páginas 20, 54, 55, 58, 127, 129, 150, 153 e 155.

BRAGINSKY; KHALILI. Quantum measurement. [S.1.]: Cambridge University Press, 1995. Citado 6 vezes nas páginas 41, 47, 53, 54, 148 e 149.

BREUER, H. P.; PETRUCCIONE, F. The Theory of Open Quantum Systems. [S.1.]: Oxford University Press, 2002. Citado 21 vezes nas páginas 8, 40, 41, 46, 47, 48, 53, 54, 89, 102, 104, 106, 111, 113, 114, 115, 116, 119, 120, 125 e 150.

BRIEGEL, H. J. et al. Measurement-based quantum computation. Nature Physics, Nature Publishing Group, p. 19-26, Jul 2009. Disponível em: < doi:10.1038/nphys1157>. Citado na página 43.

BRUN. A simple model of quantum trajectories. Aug 2001. Disponível em: <http://arxiv.org/abs/quant-ph/0108132v1>. Citado 4 vezes nas páginas 40, 41, 54 e 118. 
CALLEN; WELTON. Irreversibility and generalized noise. Jul 1951. Disponível em: <http://dx.doi.org/10.1103/PhysRev.32.110>. Citado 4 vezes nas páginas 40, 41, 48 e 89.

CARMICHAEL, H. Statistical methods in quantum optics 1 - master equations and Fooker-Planck equations. [S.l.: s.n.], 1990. Citado 11 vezes nas páginas 40, 41, 46, 47, 48, 54, 91, 92, 93, 96 e 99.

CARMICHAEL, H. An open systems approach to quantum optics. [S.l.: s.n.], 1991. Citado 9 vezes nas páginas 40, 41, 46, 47, 48, 50, 54, 96 e 100.

CAVES, C. M.; MILBURN, G. J. Quantum-mechanical model for continuous position measurements. Phys. Rev. A, American Physical Society, v. 36, p. 5543-5555, Dec 1987. Disponível em: < doi/10.1103/PhysRevA.36.5543> C Citado na página 47.

CAVES, C. M. et al. On the measurement of a weak classical force coupled to a quantummechanical oscillator. i. issues of principle. Rev. Mod. Phys., American Physical Society, v. 52, p. 341-392, Apr 1980. Disponível em: <doi:10.1103/RevModPhys.52.341>. Citado 2 vezes nas páginas 41 e 47.

CHALMERS, A. O que é ciência, afinal? [S.1.]: Editora Brasiliense, 1993. Citado na página 17.

CHAMBRION, T. et al. Controllability of the discrete-spectrum schrödinger equation driven by an external field. Annales de L'Institut Henri Poincare Section Physique Theorique, American Physical Society, v. 26, p. 329-349, Jan 2009. Disponível em: $<10.1016 /$ j.anihpc.2008.05.001>. Citado na página 53.

CHUANG, I. L. et al. Experimental realization of a quantum algorithm. Nature, Nature Publishing Group, v. 393, p. 143-146, May 1998. Disponível em: < doi:10.1038/30181>. Citado 3 vezes nas páginas 45, 108 e 109.

CLERK, A. Quantum noise and quantum measurement. In: Quantum Machines: Measurement and Control of engineered quantum systems. Les Houches - 2011: Oxford University Press, 2014. p. 61-111. Disponível em: <http://physinfo.fr/houches/pdf/ Clerk.pdf $>$. Acesso em: 16-01-2016. Citado 3 vezes nas páginas 55, 89 e 148.

CLERK, A. A. et al. Introduction to quantum noise, measurement, and amplification. Rev. Mod. Phys., American Physical Society, v. 82, p. 1155-1208, Apr 2010. Disponível em: < doi/10.1103/RevModPhys.82.1155>. Citado na página 55.

COHEN-TANNOUDJI, C.; DIU; LALOë. Quantum Mechanics Vol 1 e Vol 2. [S.l.]: John Wiley and Sons, 1977. Citado 4 vezes nas páginas 17, 27, 50 e 53.

CRUZ, J. J. da. Controle Robusto Multivariável. [S.l.]: EDUSP, 1996. Citado na página 49.

D-WAVE-SYSTEMS. Homepage. 2012. [Online; accessed 29-March-2013]. Disponível em: $<$ http://www.dwavesys.com>. Citado na página 43.

D'ALESSANDRO, D. Introduction to Quantum Control and Dynamics. [S.1.]: Chapman and Hall, 2008. Citado 13 vezes nas páginas 40, 45, 46, 50, 51, 59, 63, 64, 98, 152, 155, 156 e 159. 
DAVIES, E. B. Quantum Theory of Open Systems. [S.1.]: Academic Press, 1976. Citado 2 vezes nas páginas 51 e 53.

DAVIS, M.; VINTER, R. Stochastic Modelling and Control. [S.l.]: Chapman and Hall, 1985. Citado na página 49.

DEUTSCH, D. Quantum theory, the church-turing principle and the universal quantum computer. Proceedings of the Royal Society of London, Series A, v. 400, p. 97-117, 1985. Citado 3 vezes nas páginas 14, 42 e 43.

DEVORET et al. Quantum machines: measurement and control of engineered quantum systems. [S.1.]: Oxford University Press, 2014. Citado na página 55.

DIRAC, P. A. M. The Principles of Quantum Mechanics. [S.l.]: Oxford University Press, 1930. Citado na página 65.

DIVINCENZO. Two bit gates are universal for quantum computation. Jul 1994. Disponível em: <http://arxiv.org/abs/cond-mat/9407022v1>. Citado 3 vezes nas páginas 40, 44 e 45.

DIVINCENZO, D. P. The physical implementation of quantum computation. 2000. Disponível em: <arXiv:quant-ph/0002077v3>. Citado 2 vezes nas páginas 48 e 49.

DOHERTY; JACOBS. Feedback-control of quantum systems using continuous stateestimation. Jun 1999. Disponível em: <http://arxiv.org/abs/quant-ph/9812004v2>. Citado na página 137.

DOHERTY, A. C. et al. Quantum feedback control and classical control theory. Physical Review A, v. 62, 2000. Disponível em: < http://arxiv.org/abs/quant-ph/9912107v2>. Citado 6 vezes nas páginas 55, 127, 136, 137, 138 e 210.

EDWARDS; BELAVKIN. Optimal quantum filtering and quantum feedback control. Aug 2005. Disponível em: <http://arxiv.org/abs/quant-ph/0506018v2>. Citado na página 102.

FEYNMAN, R. P. Simulating physics with computers. International Journal of Theoretical Physics, v. 21, 1982. Citado na página 14.

FICHMANN, L.; SALluM, E. M. Sistemas Dinâmicos - Noções Básicas. [S.l.]: Publicações do IME, 2004. Citado 2 vezes nas páginas 20 e 23.

GARDINER. Handbook of Stochastic Methods. [S.1.]: Springer, 2003. Citado na página 118.

GARDINER; PARKINS; ZOLLER. Wave function quantum stochastic differential equations and quantum jump simulation methods. Oct 1992. Disponível em: <http://dx.doi.org/10.1103/PhysRevA.46.4363>. Citado 2 vezes nas páginas 103 e 129.

GARDINER; ZOLLER. Quantum Noise - A handbook of markovian and non-markovian quantum stochastic methods with applications to quantum optics. [S.1.]: Springer, 2004. Citado 10 vezes nas páginas 40, 41, 46, 47, 48, 54, 55, 92, 101 e 122.

GELFAnD, I. M.; FOMIN, S. V. Calculus of Variations. [S.l.]: Prentice Hall, 1963.

Citado 2 vezes nas páginas 20 e 23. 
GIRVIN, S. M. Circuit qed: superconducting qubits coupled to microwave photons. In: Quantum Machines: Measurement and Control of engineered quantum systems.

Les Houches - 2011: Oxford University Press, 2014. p. 113-255. Disponível em: < https: //www.chalmers.se/en/departments/mc2/education/Events/Quantum\%20Simulation\% 20and\%20Computation/Documents/Girvin_CQED_LES_HOUCHES.pdf > . Acesso em: 16-01-2016. Citado 5 vezes nas páginas 46, 48, 55, 100 e 102.

GORINI; KOSSAKOWSKI; SUDARSHAN. Completely positive dynamical semigroups of n-level systems. Journal of Mathematical Physics, v. 17, p. 821-825, 1976. Disponível em: <http://dx.doi.org/10.1063/1.522979>. Citado na página 102.

GOTTFRIED, K. Quantum Mechanics, Volume I: Fundamentals. [S.l.]: Benjamin, 1966. Citado na página 148.

GRIFFITHS, D. J. Introduction to Quantum Mechanics. [S.1.]: Pearson Prentice Hall, 2005. Citado na página 27.

HALL, E. N. Exploring Chaos: A Guide to the New Science of Disorder. [S.1.]: Springer, 1994. Citado na página 18.

HOSTEN, O. et al. Counterfactual quantum computation through quantum interrogation. Nature, Nature Publishing Group, v. 439, p. 949-952, Feb 2006. Disponível em: < doi:10.1038/nature04523>. Citado na página 43.

HUANG, G. M.; TARN, T. J. On the controlability of quantum-mechanical systems. Journal of Mathematical Physics, v. 24, 1983. Disponível em: <http: //dx.doi.org/10.1063/1.525634>. Citado 15 vezes nas páginas 40, 45, 50, 52, 59, 73, 74, $75,76,77,80,81,132,152$ e 156.

HUDSON; PARTHASARATHY. Quantum ito's formula and stochastic evolution. Communications of Mathematical Physics, Springer, v. 93, p. 301-323, 1984. Disponível em: < https://dx.doi.org/10.1007\%2FBF01258530>. Citado 2 vezes nas páginas 54 e 150.

JACOBS; STECK. A straightforward introduction to continuous quantum measurements. Nov 2006. Disponível em: <http://arxiv.org/abs/quant-ph/0611067>. Citado 6 vezes nas páginas 40, 41, 54, 55, 122 e 137.

JACOBS, K. Topics in quantum measurement and quantum noise. Tese (Doutorado) Imperial College of the University of London, 1998. Citado na página 107.

JACOBS, K. Quantum measurement theory. [S.l.: s.n.], 2014. Citado 23 vezes nas páginas 40, 41, 46, 48, 53, 54, 55, 88, 90, 95, 99, 100, 103, 122, 123, 125, 126, 127, 129, 130, 131, 137 e 155 .

JAMES; NURDIN; PETERSEN. H-infinity control of linear quantum stochastic systems. May 2007. Disponível em: < http://arxiv.org/abs/quant-ph/0703150v2>. Citado 2 vezes nas páginas 52 e 130.

JOHANSSON; NATION; NORI. Qutip: An open-source python framework for dynamics of open quantum systems. Nov 2011. Disponível em: <http: //arxiv.org/abs/1110.0573v2>. Citado na página 179. 
JOHANSSON; NATION; NORI. QuTiP - A quantum Toolbox in Python. 2012. [Online; accessed 01-outubro-2015]. Disponível em: <http://qutip.org $>$. Citado na página 179.

JOHANSSON; NATION; NORI. Qutip 2: A python framework for the dynamics of open quantum systems. Nov 2012. Disponível em: < http://arxiv.org/abs/1211.6518v1>. Citado na página 179.

JURDJEVIC, V. Geometric Control Theory. [S.l.]: Cambridge, 1997. Citado 6 vezes nas páginas 14, 25, 26, 49, 51 e 85.

KAILATH, T. Linear Systems. [S.l.]: Prentice-Hall, 1980. Citado 2 vezes nas páginas 49 e 61.

KAMPEN van. Stochastic Processes in Physics and Chemistry. [S.l.]: North Holland, 1992. Citado na página 90.

KARIMI et al. Investigating the performance of an adiabatic quantum optimization processor. ArXiv, 2011. Disponível em: < arXiv:1006.4147v4[quant-ph]>. Citado na página 43.

KARWOWSKI; VILELA-MENDES. Quantum control in infinite dimensions. Physics Letters A, v. 322, n. 05-06, p. 282-285, 2004. Disponível em: <http: //dx.doi.org/10.1016/j.physleta.2004.01.031>. Citado 7 vezes nas páginas 59, 71, 72, 85, 132, 156 e 157.

KIESSLICH, G. et al. Charge qubit purification by an electronic feedback loop. Phys. Rev. Lett., American Physical Society, v. 107, p. 050501, Jul 2011. Disponível em: $<$ doi:10.1103/PhysRevLett.107.050501>. Citado na página 17.

KIRK, D. Optimal Controle Theory: an introduction. [S.1.]: Dover, 2004. Citado na página 49.

KNILL; LAFLAMME; MILBURN. A scheme for efficient quantum computation with linear optics. Jan 2001. Disponível em: <http://dx.doi.org/10.1038/35051009>. Citado na página 84.

KNILL, E. Quantum computing with realistically noisy devices. Nature, Nature Publishing Group, v. 434, p. 39-44, Mar 2005. Disponível em: <http://dx.doi.org/10. 1038/nature03350>. Citado 4 vezes nas páginas 43, 48, 65 e 109.

KNILL, E. Quantum computing. Nature, Nature Publishing Group, v. 463, p. 441-443, Jan 2010. Disponível em: <http://dx.doi.org/10.1038/463441a>. Citado na página 45.

KUHN, T. The Structure of Scientific Revolutions. [S.l.]: University of Chicago Press, 1970. Citado na página 17.

LADD, T. D. et al. Quantum computers. Nature, v. 464, 2010. Disponível em: <http://dx.doi.org/10.1038/nature08812>. Citado 4 vezes nas páginas 43, 45, 48 e 109.

LAGANA, A. A.; LOHE, M. A.; SMEKAL, L. von. Construction of a universal quantum computer. Phys. Rev. A, American Physical Society, v. 79, p. 052322, May 2009. Disponível em: < doi/10.1103:PhysRevA.79.052322>. Citado na página 42. 
LANCZOS, C. The Variational Principles of Mechanics. [S.l.]: University of Toronto Press, 1949. Citado na página 23.

LEITãO, A. C. L. G.; BAUMEISTER, J. Introdução à teoria de controle e programação dinâmica. [S.l.]: IMPA, 2014. Citado na página 49.

LEVINE, J. Analysis and Control of Nonlinear Systems. [S.l.]: Springer, 2009. Citado 4 vezes nas páginas 49, 59, 60 e 61 .

LISBOA, A. C. Controle quântico ótimo:Fundamentos, aplicações e extensões da teoria. Tese (Doutorado) - Escola Politécnica da Universidade de São Paulo, 2015. Citado 6 vezes nas páginas 27, 50, 51, 59, 152 e 156.

LLOYD, S.; BRAUNSTEIN, S. L. Quantum computation over continuous variables. Phys. Rev. Lett., American Physical Society, v. 82, p. 1784-1787, Feb 1999. Disponível em: <doi:10.1103/PhysRevLett.82.1784>. Citado 3 vezes nas páginas 43, 44 e 84.

MABUCHI. Real-time feedback control of quantum optical input-output systems. In: Quantum Machines: Measurement and Control of engineered quantum systems. Les Houches - 2011: Oxford University Press, 2014. p. 61-111. Acesso em: 16-01-2016. Citado 4 vezes nas páginas 20, 55, 128 e 129.

MADRID, R. de la. The role of the rigged hilbert space in quantum mechanics. European Journal of Physics, v. 26, p. 287-312, 2005. Disponível em: <http: //dx.doi.org/10.1088/0143-0807/26/2/008>. Citado na página 80.

MARTINEZ et al. Teoria dos Números. [S.1.]: IMPA, 2015. Citado 3 vezes nas páginas 14,39 e 44.

MATSUI, T. Note on bulk quantum turing machine. Nov. 2004. Citado na página 42.

MATTHEWS, J. C. F. Quantum optics: an entangled walk of photons. Nature, p. 47-48, 2012. Disponível em: < doi:10.1038/nature11035>. Citado na página 43.

MAYMIN, P. The lambda-q calculus can efficiently simulate quantum computers. Feb 1997. Citado na página 44.

MILnE, D. F.; KOROLKOVA, N. V. Composite-cluster estados e arquiteturas alternativas para um caminho computação quântica. Phys. Rev. A, American Physical Society, v. 85, p. 032319, Mar 2012. Disponível em: < doi:10.1103/PhysRevA.85.032310>. Citado 2 vezes nas páginas 17 e 43.

MISZCZAK, J. A. Models of quantum computation and quantum programming languages. Bull. Pol. Acad. Sci-Tech. Sci., v. 59, p. 305-324, Dec 2010. Disponível em: $<$ doi:10.2478/v10175-011-0039-5>. Citado na página 44.

NELSON, E. Analytic vectors. Annals of Mathematics, v. 70, 1959. Citado 3 vezes nas páginas 52,73 e 74 .

NEUMAIER, A.; WESTRA, D. Classical and Quantum Mechanics via Lie algebras. [S.l.: s.n.], 2008. Citado na página 62.

NIELSEN, M. A. A geometric approach to quantum circuit lower bounds. Feb 2005.

Citado na página 43. 
NIELSEN, M. A.; CHUANG, I. L. Quantum Computation and Quantum Information. [S.1.]: Cambridge, 2000. Citado 8 vezes nas páginas 14, 29, 42, 44, 45, 48, 53 e 57.

NIELSEN, M. A. et al. Quantum computation as geometry. Science, v. 311, 2006. Disponível em: <http://arxiv.org/abs/quant-ph/0603161v2>. Citado 2 vezes nas páginas 43 e 51.

NISHIMURA, H.; OZAWA, M. Perfect computational equivalence between quantum turing machines and finitely generated uniform quantum circuit families. Nov 2005. Citado na página 43.

NURDIN; JAMES; DOHERTY. Network synthesis of linear dynamical quantum stochastic systems. Jan 2010. Disponível em: <http://arxiv.org/abs/0806.4448v2>. Citado na página 128.

NURDIN; JAMES; PETERSEN. Coherent quantum lqg control. Automatica, Elsevier, v. 45, p. 1837, 2009. Disponível em: <http://arxiv.org/abs/0711.2551v2>. Citado na página 132.

OLIVER, W. D. Quantum physics: Keep your feet on the ground. Nature, Nature Publishing Group, v. 473, p. 164-165, May 2011. Disponível em: < doi:10.1038/473164a>. Citado na página 43.

OZAWA, M. Quantum nondemolition monitoring of universal quantum computers. Phys. Rev. Lett., American Physical Society, v. 80, p. 631-634, Jan 1998. Disponível em: $<$ doi:10.1103/PhysRevLett.80.631>. Citado na página 43.

PARTHASARATHY. An introduction to quantum stochastic calculus. [S.1.]: Springer Basel, 1992. Citado 3 vezes nas páginas 54, 150 e 155.

PRESKILL, J. Fault-tolerant quantum computation. Dec 1997. Citado 10 vezes nas páginas 43, 48, 51, 53, 65, 104, 110, 116, 117 e 166.

PRESKILL, J. Quantum Information and Computation - Lecture Notes. [S.1.]: Caltech, 1998. Citado na página 88.

PRUGOVECKI, E. Quantum Mechanics in Hilbert Space. [S.1.]: Dover, 1981. Citado 14 vezes nas páginas 40, 50, 51, 52, 58, 65, 66, 67, 68, 78, 131, 132, 153 e 154.

PURI, R. Mathematical Methods of Quantum Optics. [S.l.]: Springer, 2001. Citado 2 vezes nas páginas 47 e 50.

RAMAKRISHNA et al. Controllability of molecular systems. Physical Review A, v. 51, n. 2, Feb 1995. Disponível em: < http://dx.doi.org/10.1103/PhysRevA.51.960>. Citado 5 vezes nas páginas 50, 51, 131, 132 e 152 .

RAUSSENDORF, R.; HARRINGTON, J. Fault-tolerant quantum computation with high threshold in two dimensions. Phys. Rev. Lett., American Physical Society, v. 98, p. 190504, May 2007. Disponível em: < doi:10.1103/PhysRevLett.98.190504>. Citado na página 43.

REED; SIMON. Functional Analysis. [S.1.]: Academic Press, 1980. Citado 10 vezes nas páginas 40, 50, 51, 52, 69, 70, 86, 117, 132 e 154 . 
RIESZ; SZ.-NAGY. Functional Analysis. [S.l.]: Dover, 1990. Citado na página 154.

RODó, C. S. Quantum information with continuous variable systems. Phys. Rev. B, Lambert Academic Publishing (LAP, v. 82, p. 094511, Sep 2011. Citado na página 43.

SAKURAI, J. J. Modern Quantum Mechanics. [S.1.]: Addison Wesley Longman, 1994. Citado 11 vezes nas páginas 27, 50, 51, 53, 57, 84, 99, 105, 131, 148 e 150.

SCHUSTER, D. I. Circuit Quantum Electrodynamics. Tese (Doutorado) - Yale University, 2007. Citado 2 vezes nas páginas 46 e 47.

SCULLY; ZUBAIRY. Quantum Optics. [S.l.]: Cambridge University Press, 1997. Citado 3 vezes nas páginas 50,56 e 99 .

SHOR, P. A scheme for reducing dedocerence in quantum computer memory. Physical Review A, American Physical Society, v. 52, n. 4, p. R2493-R2496, Oct 1995. Disponível em: <http://link.aps.org/doi/10.1103/PhysRevA.52.R2493>. Citado na página 48.

SHOR, P. W. Algorithms for quantum computation: Discrete logarithms and factoring. Symposium on Foundations of Computer Science, 1994. Citado 2 vezes nas páginas 14 e 44.

SHOR, P. W. Polynomial-time algorithms for prime factorization and discrete logarithms on a quantum computer. SIAM Journal of Computation, 1997. Citado na página 14.

SOTOMAYOR, J. Lições de Equações Diferenciais Ordinárias. [S.l.]: IMPA, 1980. Citado na página 20.

TONDER, A. v. A lambda calculus for quantum computation. SIAM J. Comput., p. 1109-1135, Apr 2004. Disponível em: < doi:10.1137/S0097539703432165>. Citado na página 44.

TRAUB, J. F. A continuous model of computation. Physics Today, p. 39-43, May 1999. Citado na página 43.

VILELA-MENDES, R. Universal families and quantum control in infinite dimensions. Physics Letters A, v. 373, n. 30, p. 2529-2532, 2009. Disponível em: < http:

//dx.doi.org/10.1016/j.physleta.2009.05.014>. Citado 6 vezes nas páginas 59, 72, 85, 132,156 e 157.

VILELA-MENDES, R.; MANIKO, V. I. On the problem of quantum control in infinite dimensions. Journal of Physics A: Mathematical and Theoretical, v. 44, n. 13, 2011. Disponível em: <http://dx.doi.org/10.1016/j.physleta.2009.05.014>. Citado 5 vezes nas páginas 59, 85, 132, 156 e 157.

WALLS; MILBURN. Quantum Optics. [S.1.]: Springer, 2008. Citado 4 vezes nas páginas $47,50,56$ e 99 .

WALTHER, P. et al. Experimental one-way quantum computing. Nature, Nature Publishing Group, v. 434, p. 169-176, Mar 2005. Disponível em: < doi:10.1038/ nature03347>. Citado na página 43.

WILCE, A. Quantum logic and probability theory. Stanford Encyclopedia of Philosophy, Stanford University, Sep 2012. Disponível em: < http://plato.stanford.edu/archives/ fall2012/entries/qt-quantlog/>. Citado na página 58. 
WISEMAN. Quantum trajectories and quantum measurement theory. Feb 2003. Disponível em: <http://arxiv.org/abs/quant-ph/0302080v1>. Citado 2 vezes nas páginas 36 e 119.

WISEMAN; MILBURN. Quantum measurement and Control. [S.l.]: Cambridge University Press, 2010. Citado 30 vezes nas páginas 40, 41, 46, 47, 48, 50, 52, 53, 54, 55, 56, 84, 89, 90, 93, 95, 96, 97, 99, 103, 119, 122, 126, 127, 129, 130, 131, 132, 150 e 155.

WISEMAN, H. M. Quantum theory of continuous feedback. Phys. Rev. A, American Physical Society, v. 49, p. 2133-2150, Mar 1994. Disponível em: $<$ doi/10.1103/PhysRevA.49.2133>. Citado 2 vezes nas páginas 47 e 56.

WISEMAN, H. M. Using feedback to eliminate back-action in quantum measurements. Phys. Rev. A, American Physical Society, v. 51, p. 2459-2468, Mar 1995. Disponível em: $<$ doi:10.1103/PhysRevA.51.2459>. Citado 3 vezes nas páginas 41, 47 e 56.

WISEMAN, H. M.; MILBURN, G. J. Quantum theory of optical feedback via homodyne detection. Phys. Rev. Lett., American Physical Society, v. 70, p. 548-551, Feb 1993. Disponível em: < doi/10.1103/PhysRevLett.70.548>. Citado 2 vezes nas páginas 47 e 56.

WISEMAN, H. M.; MILBURN, G. J. Squeezing via feedback. Phys. Rev. A, American Physical Society, v. 49, p. 1350-1366, Feb 1994. Disponível em: $<$ doi/10.1103/PhysRevA.49.1350>. Citado 2 vezes nas páginas 47 e 56.

WU, R.-B.; TARN, T.-J.; LI, C.-W. Smooth controllability of infinite-dimensional quantum-mechanical systems. Phys. Rev. A, American Physical Society, v. 73, p. 012719, Jan 2006. Disponível em: <http://dx.doi.org/10.1103/PhysRevA.73.012719>. Citado 18 vezes nas páginas 40, 45, 51, 52, 59, 76, 77, 78, 80, 81, 82, 83, 84, 86, 132, 152, 154 e 156. 
Anexos 


\section{ANEXO A - Bilioteca de simulação numérica - QuTiP}

\section{A.1 Introdução}

Apresentamos a seguir os resultados da implementação dos exemplos de familiarização apontados pelos desenvolvedores da biblioteca de simulação de equações mestras determinísticas e estocásticas denominada QuTiP (JOHANSSON; NATION; NORI, 2011; JOHANSSON; NATION; NORI, 2012b). Como se poderá observar nos exemplos abaixo, a biblioteca possui funções que realizam diversos cálculos de interesse na simulação de sistemas quânticos abertos e de processamento quântico de informação.

Infelizmente, o desenvolvimento da biblioteca foi descontinuado. No entanto, seu código ainda permanece bastante útil. Informações a respeito do uso da biblioteca em simulações de sistemas abertos e fechados, simples ou complexas, com ou sem processos de medição contínua, podem ser encontradas no sítio eletrônico da biblioteca (JOHANSSON; NATION; NORI, 2012a). São providos vários exemplos de código e vários tutoriais, que demonstram passo a passo, com explicações detalhadas, a utilização das funções e sua composição. O código utilizado para simular o sistema com com medição apresentado no Capítulo 4 foi adaptado a partir do exemplo de utilização da medição de quadratura por detecção homodina. A técnica avançada de composição de superoperadores, necessária para implementar a equação mestra com realimentação, também se encontra documentada no material online.

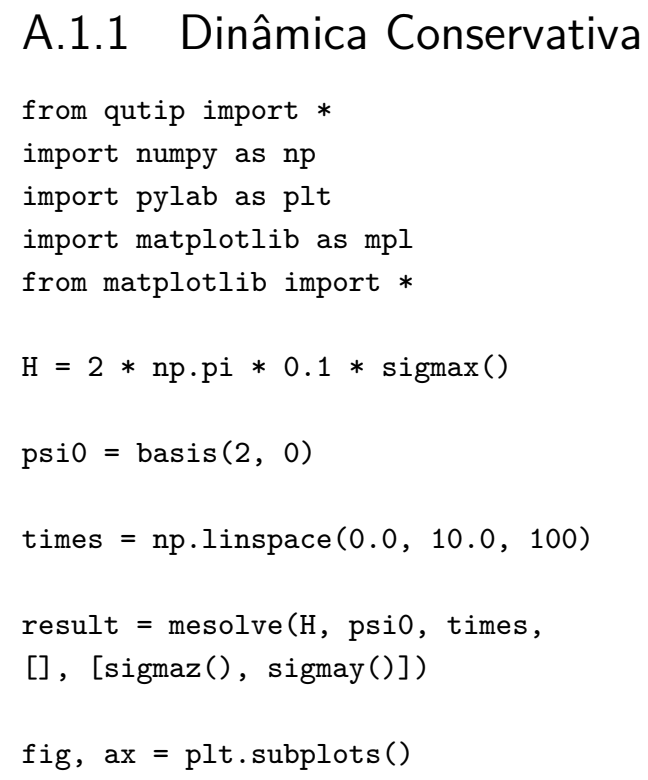


Figura 19 - Dinâmica unitária

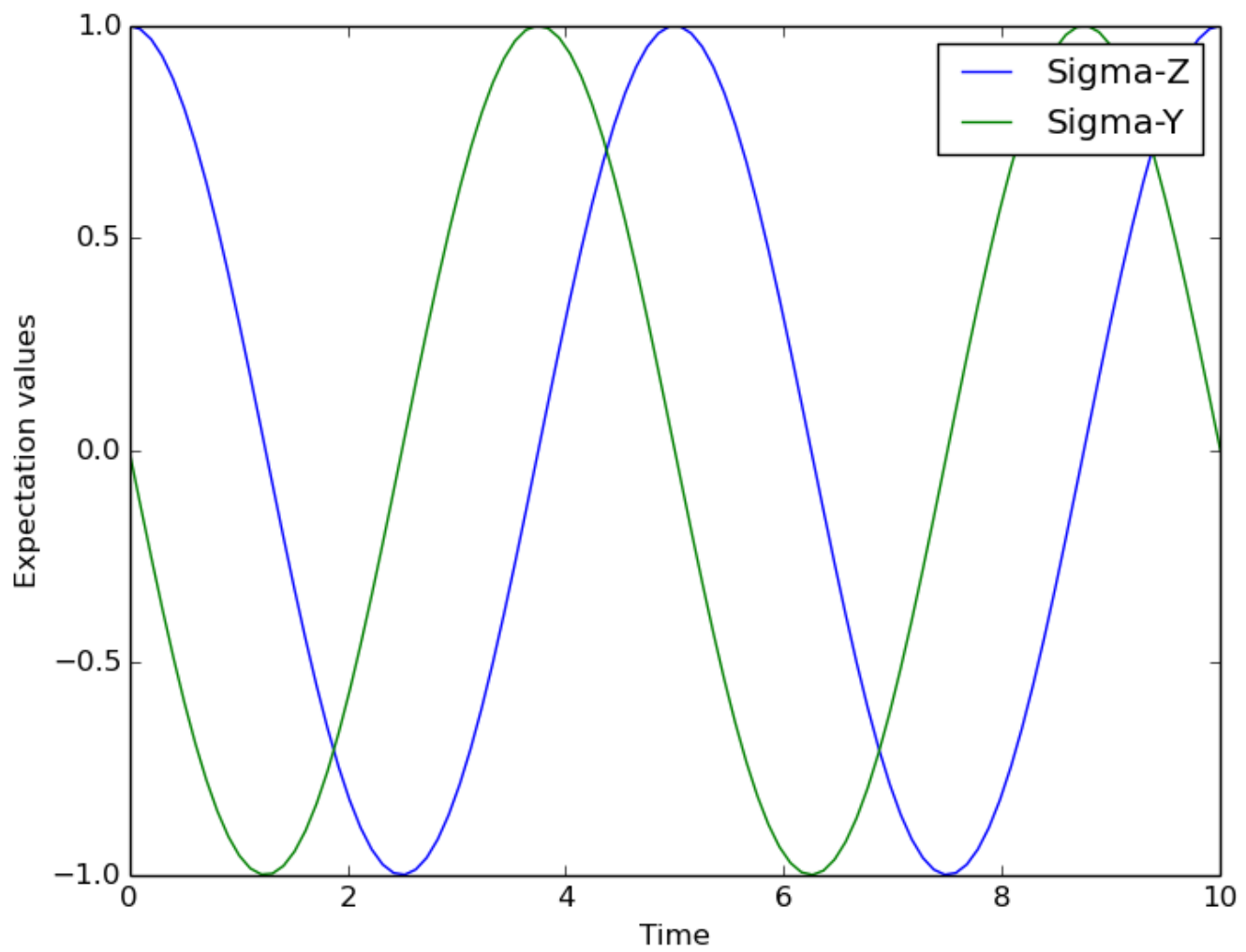

ax.plot (result.times, result.expect [0]);

ax.plot(result.times, result.expect [1]);

ax.set_xlabel('Time');

ax.set_ylabel ('Expectation values');

ax.legend (("Sigma-Z", "Sigma-Y"));

plt.show()

\section{A.1.2 Dinâmica Dissipativa}

from qutip import *

import numpy as $\mathrm{np}$

import pylab as plt

import matplotlib as mpl

from matplotlib import *

$\mathrm{H}=2 * \mathrm{np} \cdot \mathrm{pi} * 0.1 * \operatorname{sigmax}()$

$\operatorname{psi0}=\operatorname{basis}(2,0)$

times $=n p$. linspace $(0.0,10.0,100)$ 
Figura 20 - Dinâmica dissipativa

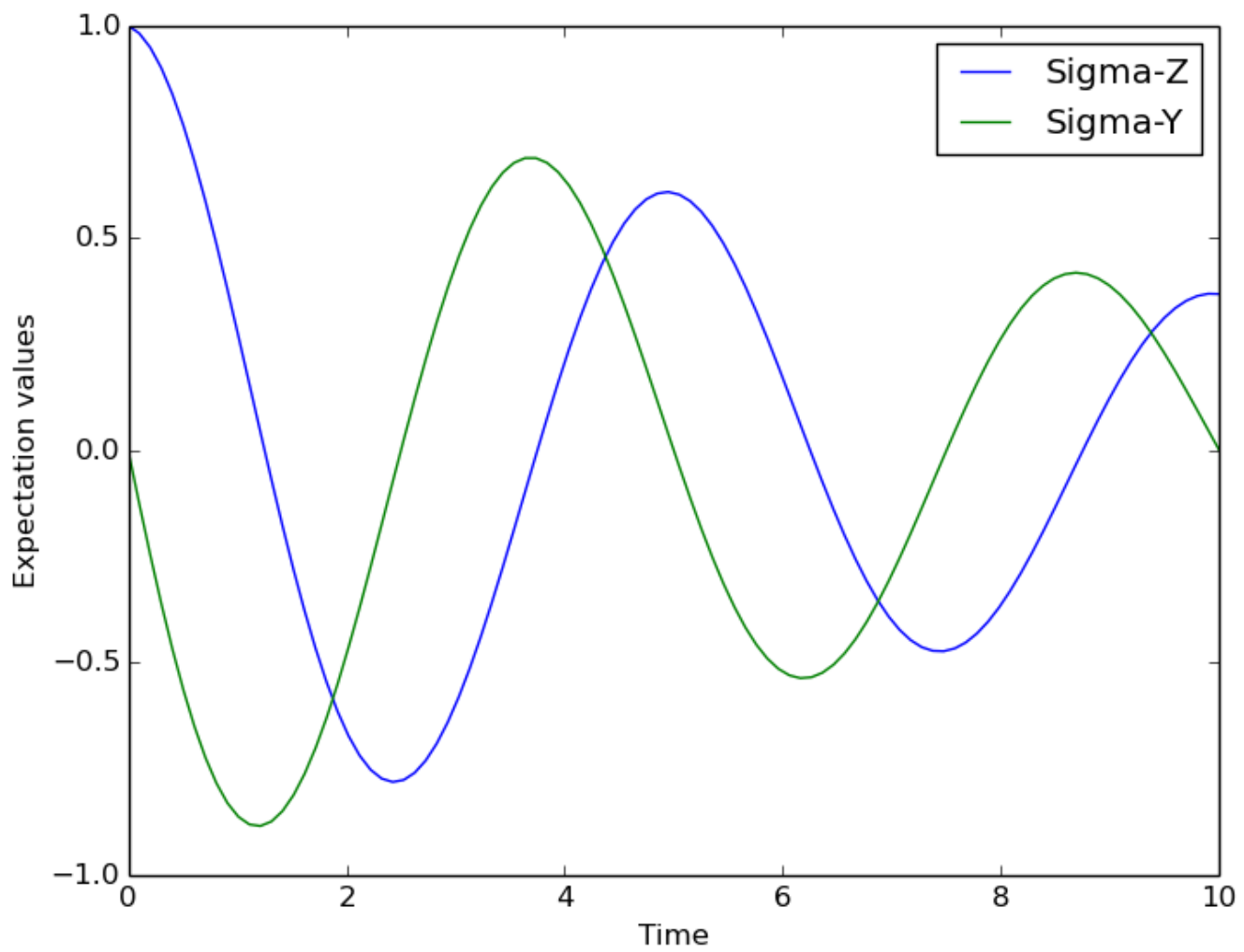

result $=$ mesolve $(\mathrm{H}$, psi0, times,

$[$ np.sqrt $(0.05) * \operatorname{sigmax}()],[\operatorname{sigmaz}(), \operatorname{sigmay}()])$

fig, ax = plt.subplots ()

ax.plot(result.times, result.expect [0]);

ax.plot (result.times, result.expect [1]);

ax.set_xlabel('Time');

ax.set_ylabel('Expectation values');

ax.legend(("Sigma-Z", "Sigma-Y"));

plt.show()

\section{A.2 Exemplos da versão 1}

\section{A.2.1 Exemplo 01 - Valores esperados e ground-states}

from qutip import *

from numpy import *

from pylab import *

\#import matplotlib as $\mathrm{mpl}$

\#from matplotlib import * 
Figura 21 - Valores esperados para o qubit e para a cavidade

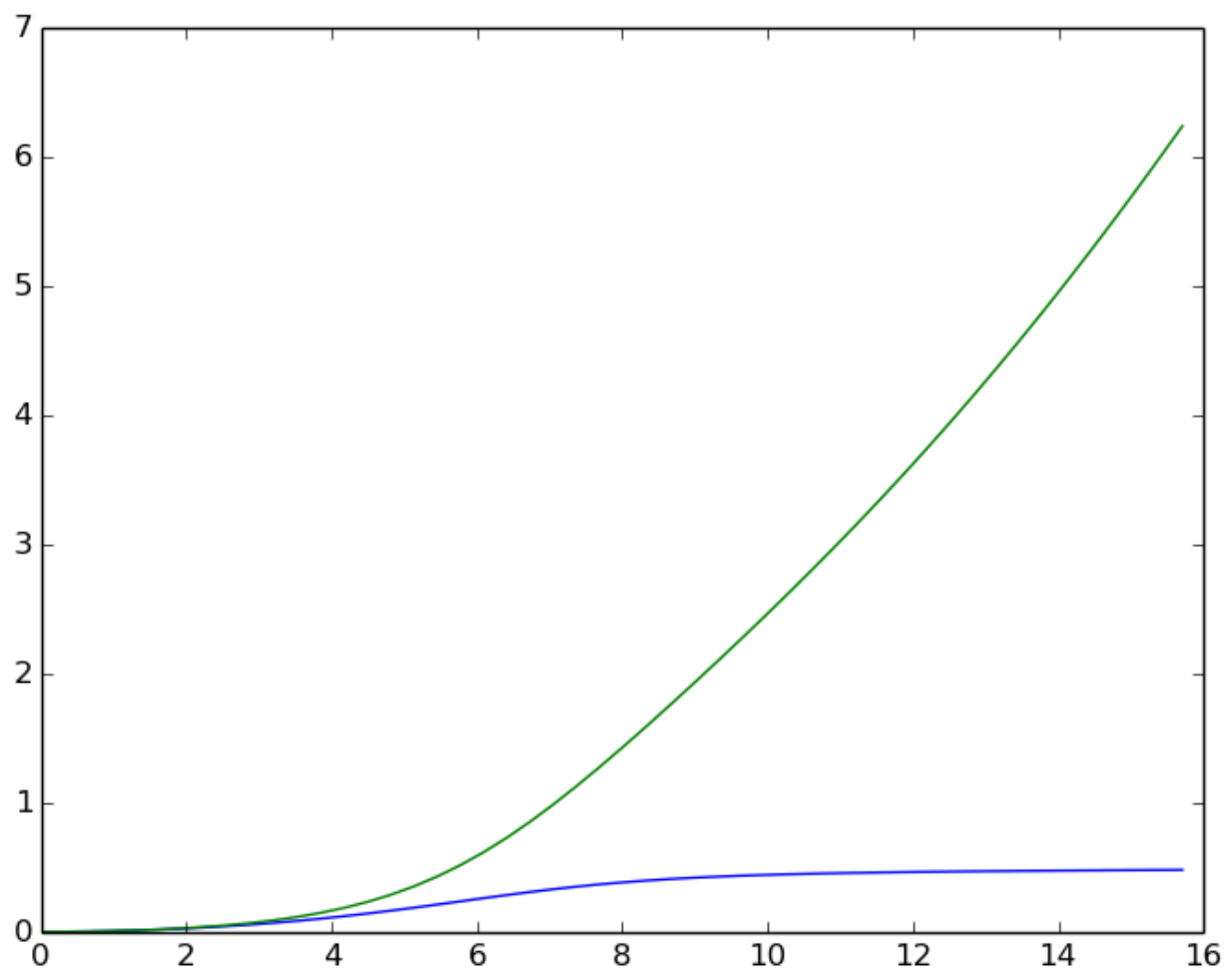

\#\# set up the calculation \#\#

$\mathrm{wc}=1.0 * 2 * \mathrm{pi} \#$ cavity frequency

wa $=1.0 * 2 *$ pi \# atom frequency

$\mathrm{N}=20$ \# number of cavity states

$\mathrm{g}=1$ inspace $(0,2.5,50) * 2 * \mathrm{pi} \#$ coupling strength vector

\#\# create operators \#\#

$\mathrm{a}=\operatorname{tensor}(\operatorname{destroy}(\mathrm{N})$, qeye (2))

$\mathrm{sm}=\operatorname{tensor}($ qeye $(\mathrm{N})$, destroy (2))

$\mathrm{nc}=\mathrm{a} \cdot \operatorname{dag}() * \mathrm{a}$

na $=\mathrm{sm} \cdot \operatorname{dag}() * \mathrm{sm}$

\#\# initialize output arrays \#\#

na_expt $=\operatorname{zeros}(\operatorname{len}(\mathrm{g}))$

$n c_{-} \operatorname{expt}=\operatorname{zeros}(\operatorname{len}(\mathrm{g}))$

print " na.isoper $=\% \mathrm{~d}$ " $\%$ na.isoper

print " nc.isoper $=\% \mathrm{~d}$ " $\%$ nc.isoper

\#\# run calculation \#\#

for $k$ in range (len $(g))$ :

\#\# recalculate the hamiltonian for each value of $g$ \#\#

$\mathrm{H}=\mathrm{wc} * \mathrm{nc}+\mathrm{wa} * \mathrm{na}+\mathrm{g}[\mathrm{k}] *(\mathrm{a} \cdot \operatorname{dag}()+\mathrm{a}) *(\mathrm{sm}+\mathrm{sm} \cdot \operatorname{dag}())$

\#\# find the groundstate \#\#

evals, ekets = H.eigenstates ()

psi_gnd $=\operatorname{ekets}[0]$ 
Figura 22 - Função de Wigner da cavidade

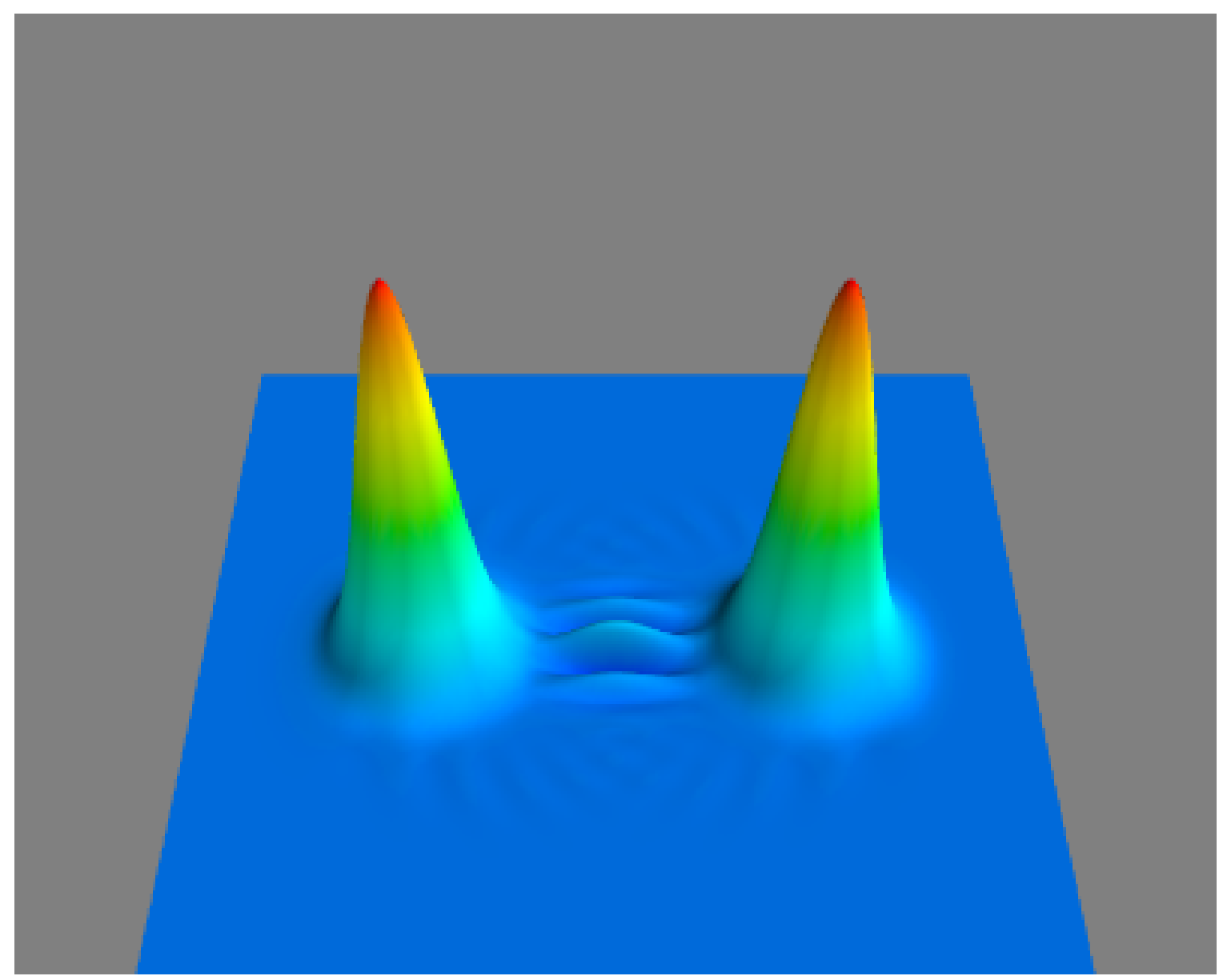

\#\# expectation values \#\#

na_expt $[\mathrm{k}]=\operatorname{expect}(\mathrm{na}, \mathrm{psi}$ _gnd) \# qubit occupation

$\mathrm{nc}$ _expt $[\mathrm{k}]=\operatorname{expect}(\mathrm{nc}, \mathrm{psi}$ gnd) $\#$ cavity occupation

plot (g, na_expt, g, nc_expt)

show()

\#\# Calculate Wigner function for coupling $g=2.5$ \#\#

rho_cavity $=$ ptrace $($ psi_gnd,0) \# trace out qubit

$\mathrm{xvec}=$ linspace $(-7.5,7.5,200)$

$\mathrm{yvec}=\mathrm{xvec}$

\#\# Wigner function \#\#

$\mathrm{W}=$ wigner (rho_cavity, $\mathrm{xvec}, \mathrm{xvec}$ )

\#\# new function calls \#\#

\# store Wigner function to file

file_data_store ("wigner.dat", W, numtype='real')

\# load input data from file

input_data $=f i l e \_d a t a \_r e a d($ 'wigner.dat')

\# plot using mayavi

from mayavi.mlab import * 
$X, Y=\operatorname{meshgrid}(\mathrm{xvec}, \mathrm{yvec})$

$\operatorname{surf}(x v e c$, yvec, input_data, warp_scale='auto')

view $(0,45)$

show()

\section{A.2.2 Exemplo 02 - Trajetorias quânticas}

Figura 23 - Trajetórias quânticas e sua dinâmica média

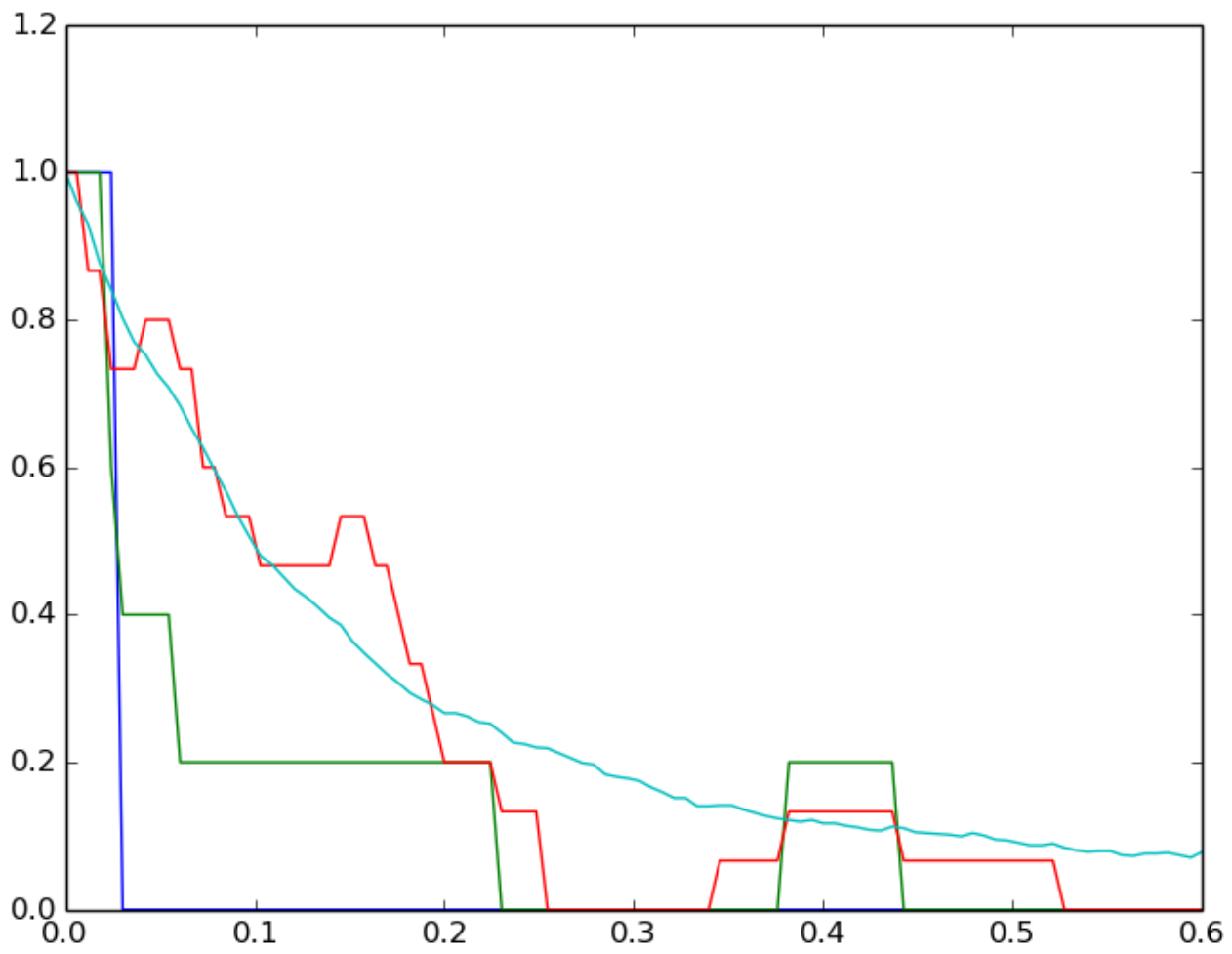

from qutip import *

from numpy import *

from pylab import *

\#import pylab as plt

\#import matplotlib as $\mathrm{mpl}$

\#from matplotlib import *

$\mathrm{N}=5$ \# number of basis states to consider

a=destroy $(\mathrm{N})$ \# cavity destruction operator

$\mathrm{H}=\mathrm{a} \cdot \operatorname{dag}() * \mathrm{a} \#$ harmonic oscillator Hamiltonian

psi0=basis $(\mathrm{N}, 1)$ \# initial Fock state with one photon

kappa=1.0/0.129 \# coupling to heat bath 
$\mathrm{nth}=0.063 \#$ temperature with $\langle\mathrm{n}\rangle=0.063$

\#\# collapse operators \#\#

c_op_list $=[]$

\#\# decay operator \#\#

c_op_list.append (sqrt (kappa $*(1+n t h)) * a)$

\#\# excitation operator \#\#

C_op_list.append (sqrt (kappa $*$ nth) $*$ a.dag ()$)$

\#\# run simulation \#\#

ntraj=904 \# number of MC trajectories

tlist=linspace $(0,0.6,100)$

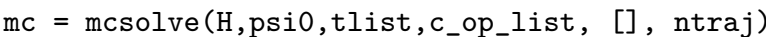

me $=$ odesolve(H,psi0,tlist,c_op_list, $[a \cdot \operatorname{dag}() * a])$

times $=$ mc.times

\#\# expectation values \#\#

$\operatorname{ex1}=\operatorname{expect}(\operatorname{num}(\mathrm{N}), \mathrm{mc} \cdot$ states $[0])$

ex5 $=\operatorname{sum}([\operatorname{expect}(\operatorname{num}(N), m c \cdot s t a t e s[k])$ for $k$ in range(5)],0)/5

$\operatorname{ex15}=\operatorname{sum}([\operatorname{expect}(\operatorname{num}(\mathrm{N}), \mathrm{mc} \cdot \operatorname{states}[\mathrm{k}])$ for $\mathrm{k}$ in range(15)],0)/15

ex904=sum ([expect (num(N),mc.states $[k])$ for $k$ in range(904)],0)/904

plot(times, ex1, times, ex5, times, ex15, times, ex904)

show()

\section{A.2.3 Exemplo 3 - Porta quântica - conservativa e dissipativa}

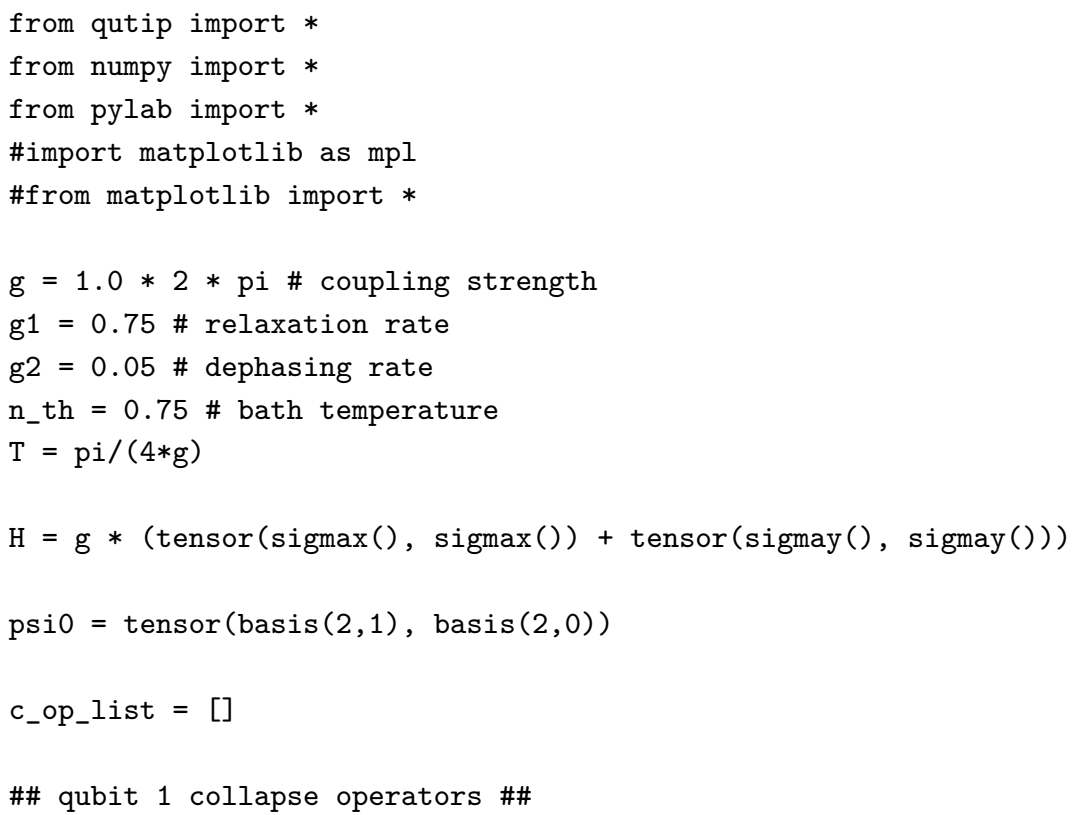


Figura 24 - Fidelidade de uma porta i-swap dissipativa
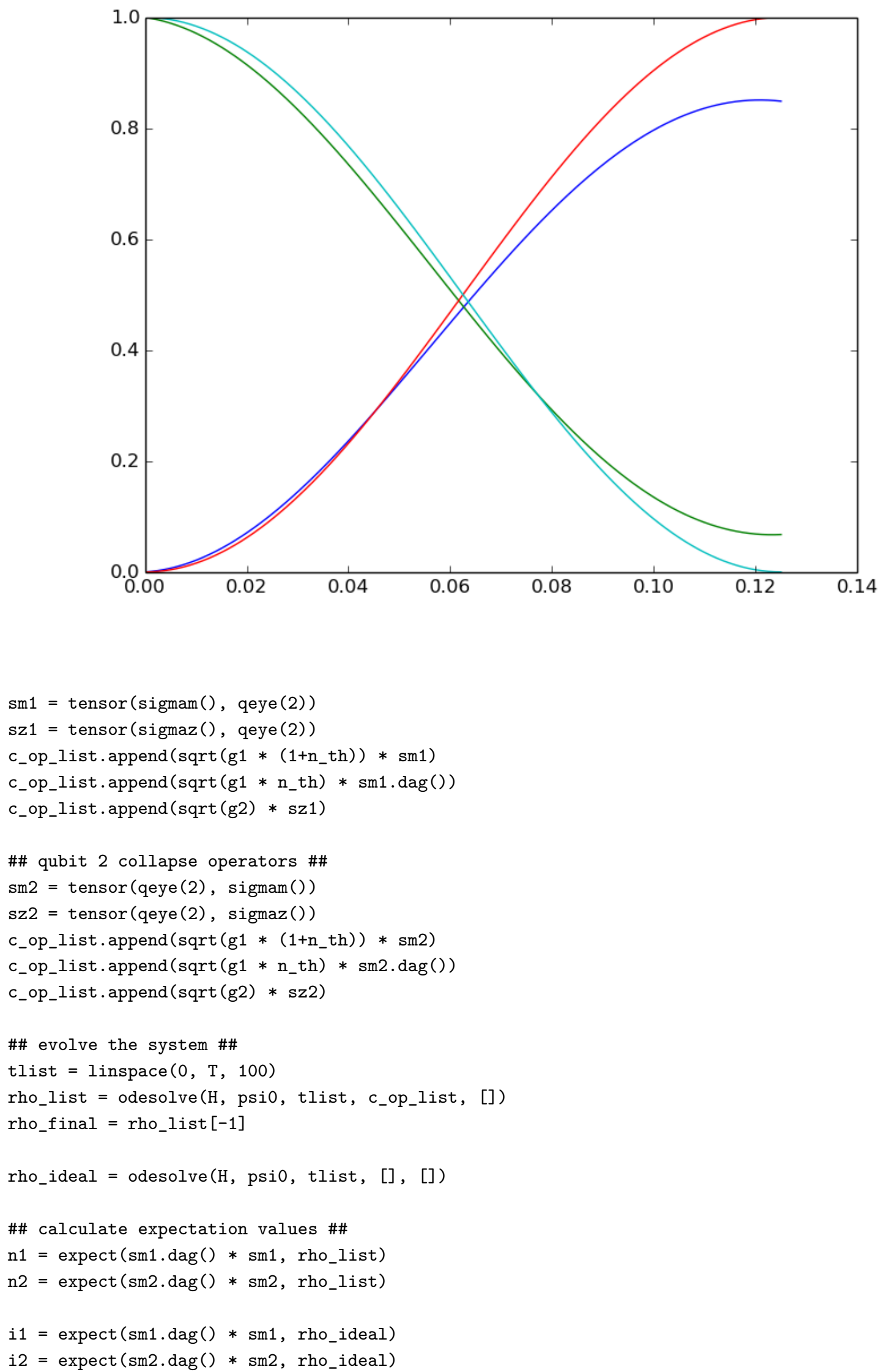
\#\# calculate the fidelity \#\#

$\mathrm{U}=(-1 \mathrm{j} * \mathrm{H} * \mathrm{pi} /(4 * \mathrm{~g})) \cdot \operatorname{expm}()$

psi_ideal $=U *$ psio

rho_ideal $=$ psi_ideal $*$ psi_ideal.dag ()

$f$ = fidelity(rho_ideal, rho_final)

print "Fidelity $=\% \mathrm{f} " \% \mathrm{f}$

plot(tlist, $\mathrm{n} 1$, tlist, $\mathrm{n} 2$, tlist, i1, tlist, i2)

show ()

\section{A.2.4 Exemplo 4 - Oscilações de Rabi}

Figura 25 - Oscilações de Rabi

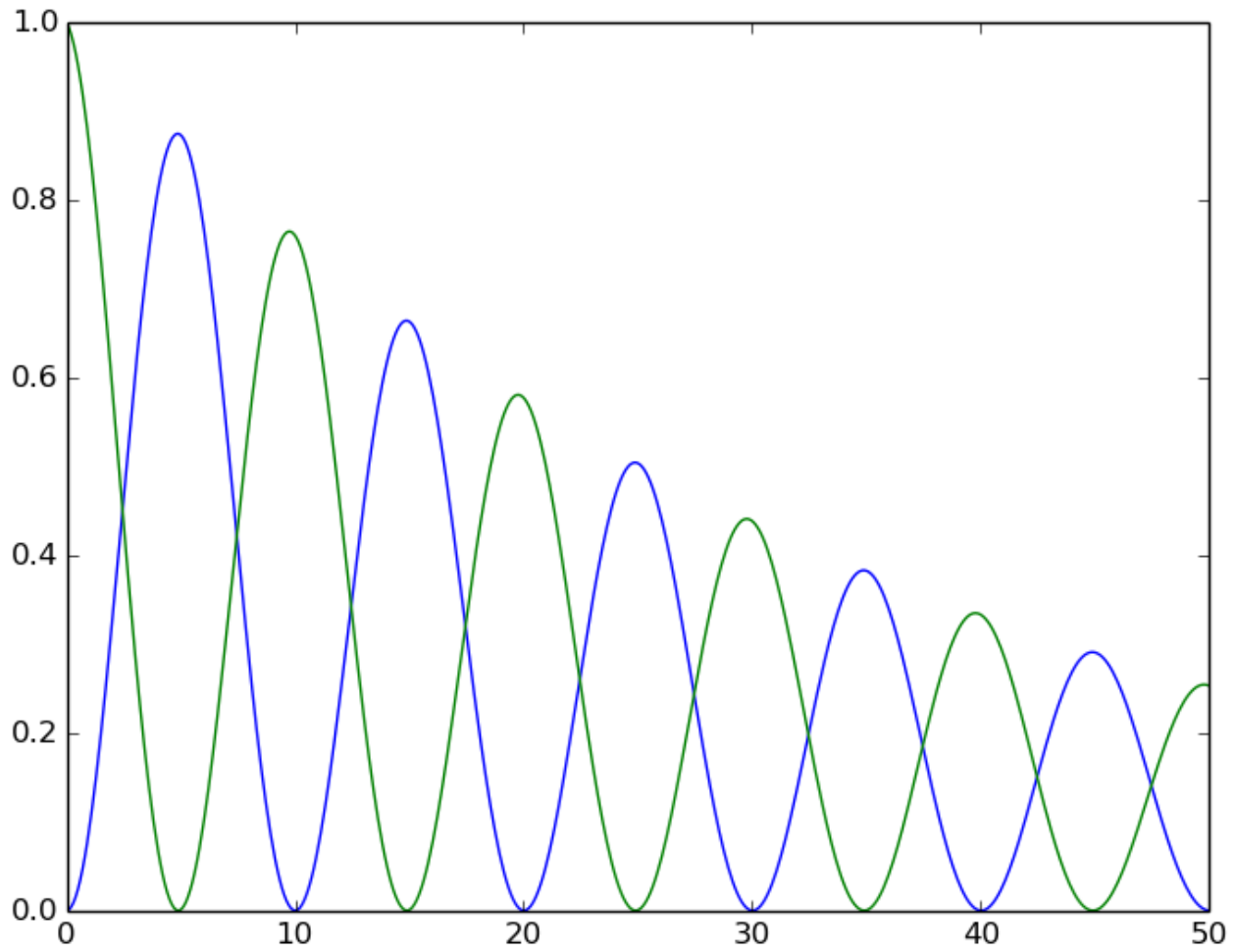

from qutip import *

from numpy import *

from pylab import *

\#from matplotlib import *

$\mathrm{N}=15 \#$ number of cavity states

$\mathrm{wc}=\mathrm{wa}=2 *$ pi $\#$ frequencies

$g=0.05 * 2 *$ pi \# coupling strength

kappa $=0.005 *$ cavity relaxation rate 


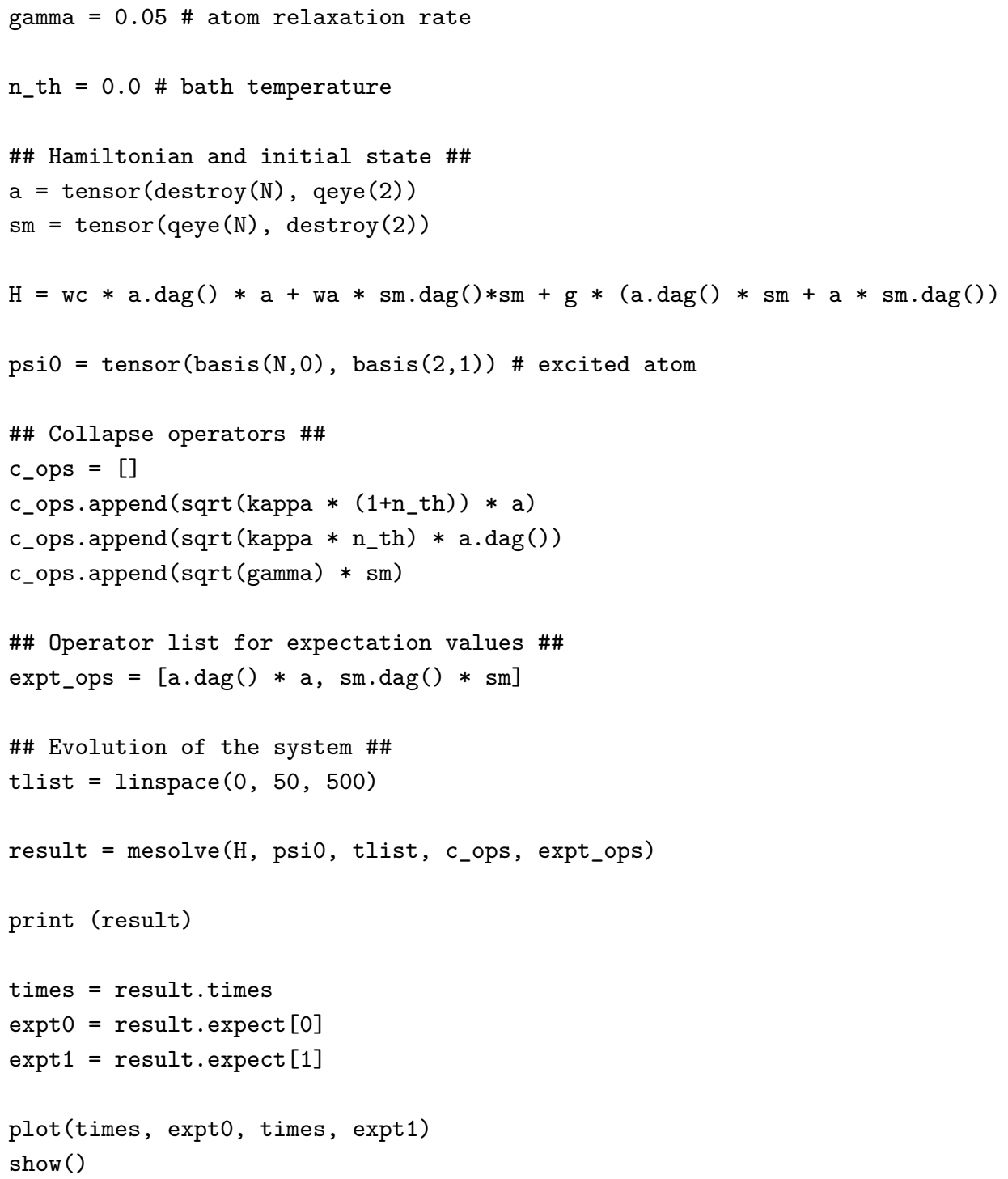

\section{A.2.5 Exemplo 5 - 3 subsistemas}

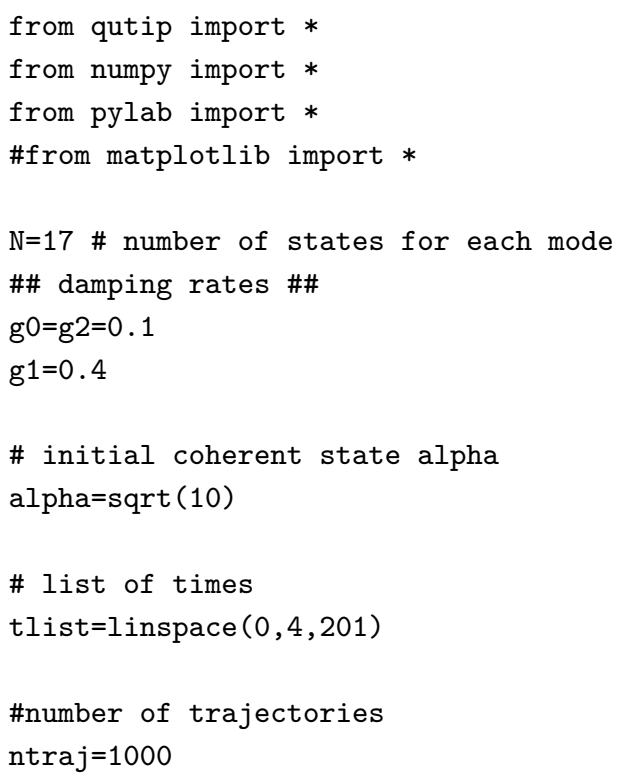


Figura 26 - Dinâmica dissipativa

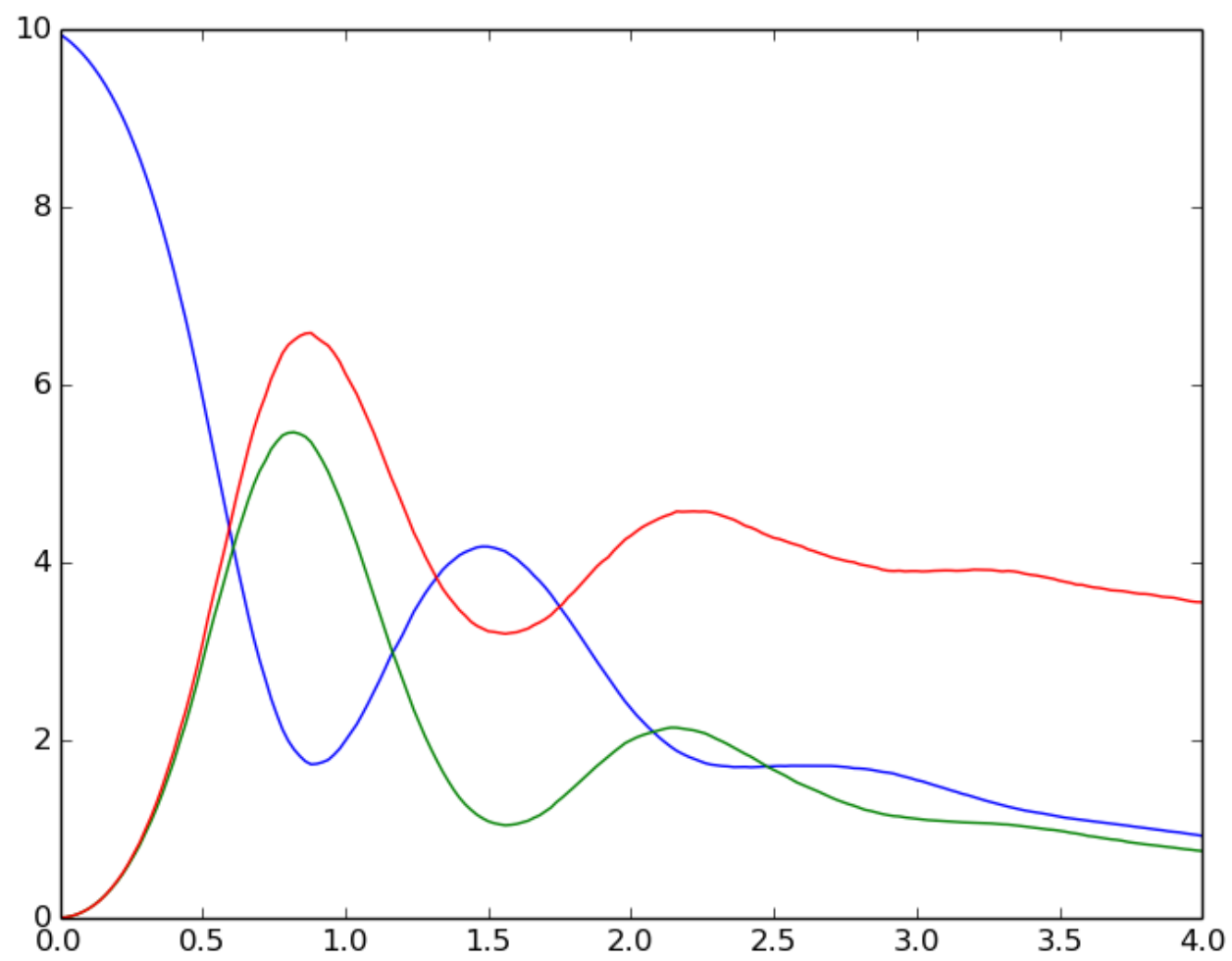

\#\# lowering operators \#\# a0=tensor $($ destroy $(N)$, qeye $(N)$, qeye $(N))$ a1=tensor $($ qeye $(N), \operatorname{destroy}(N)$, qeye $(N))$ a2=tensor $($ qeye $(N)$, qeye $(N)$, destroy $(N))$

\#\# number operators \#\#

$\mathrm{n} 0, \mathrm{n} 1, \mathrm{n} 2=[\mathrm{a} 0 \cdot \operatorname{dag}() * \mathrm{a} 0, \mathrm{a} 1 \cdot \operatorname{dag}() * \mathrm{a} 1, \mathrm{a} 2 \cdot \operatorname{dag}() * \mathrm{a} 2]$

\#\# dissipative operators \#\#

$\mathrm{C} 0, \mathrm{C} 1, \mathrm{C} 2=[\operatorname{sqrt}(2.0 * \mathrm{~g} 0) * \mathrm{a} 0, \operatorname{sqrt}(2.0 * \mathrm{~g} 1) * \mathrm{a} 1, \operatorname{sqrt}(2.0 * \mathrm{~g} 2) * \mathrm{a} 2]$

\#\# initial state \#\#

psi0=tensor $(\operatorname{coherent}(N$, alpha $), \operatorname{basis}(N, 0), \operatorname{basis}(N, 0))$

\#\# trilinear Hamiltonian \#\#

$\mathrm{H}=1 \mathrm{j} *(\mathrm{a} 0 * \mathrm{a} 1 \cdot \operatorname{dag}() * \mathrm{a} 2 \cdot \operatorname{dag}()-\mathrm{a} 0 \cdot \operatorname{dag}() * \mathrm{a} 1 * \mathrm{a} 2)$

\#\# run Monte-Carlo \#\#

avgs $=$ mcsolve $(\mathrm{H}, \mathrm{psi0}, \mathrm{tlist},[\mathrm{C0}, \mathrm{C} 1, \mathrm{C} 2],[\mathrm{n} 0, \mathrm{n} 1, \mathrm{n} 2], \mathrm{ntraj})$

\#\# run Schrodinger \#\#

reals $=$ mcsolve $(\mathrm{H}, \mathrm{psi0}, \mathrm{tlist},[],[\mathrm{n} 0, \mathrm{n} 1, \mathrm{n} 2], 1)$

times $=$ avgs.times

av0 = avgs.expect [0]

$\operatorname{av1}=\operatorname{avgs} \cdot \operatorname{expect}[1]$ 
Figura 27 - Dinâmica não-dissipativa

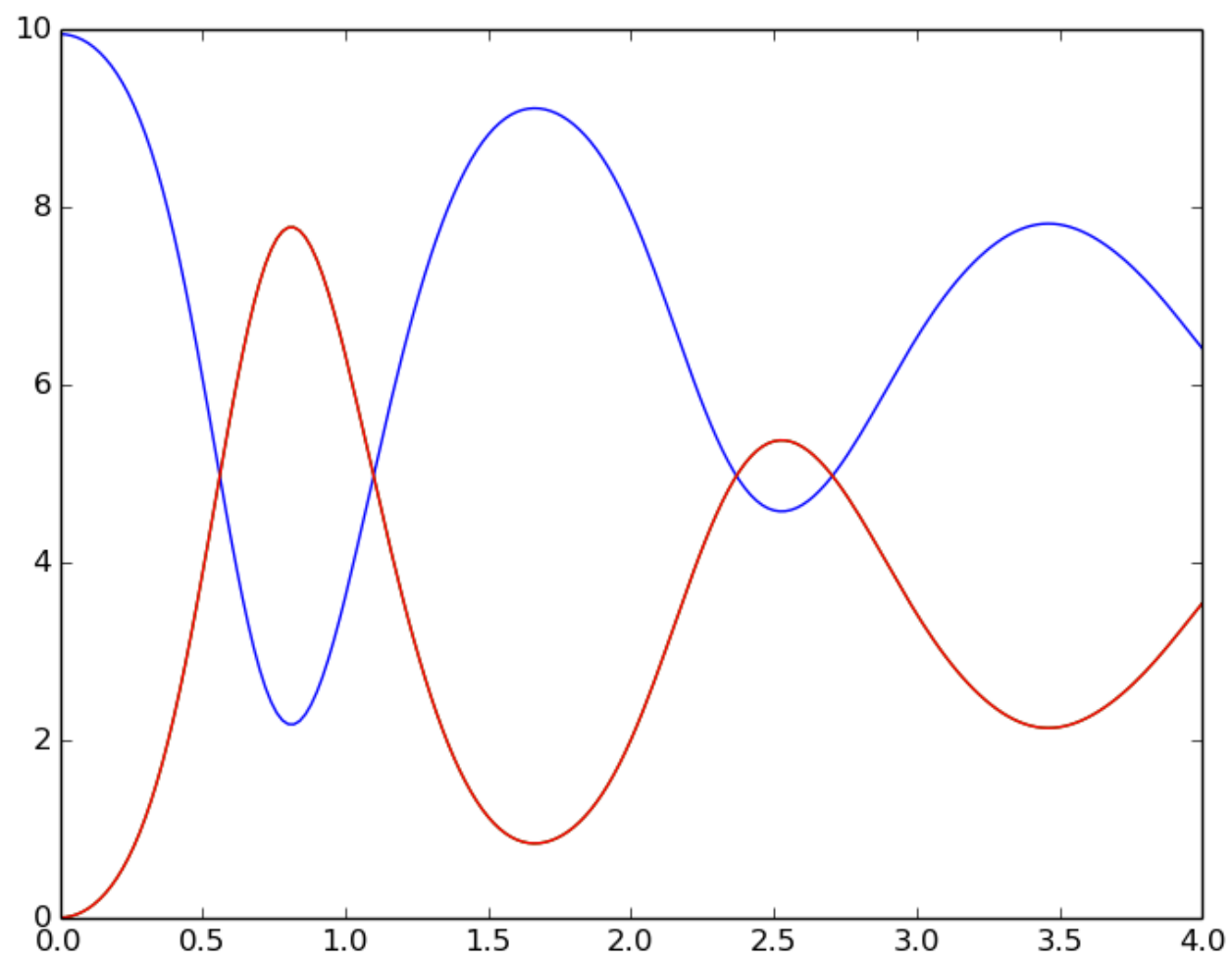

av2 = avgs.expect [2]

plot(times, av0, times, av1, times, av2)

show()

times $=$ reals.times

plot(times, reals.expect[0], times, reals.expect[1], times, reals.expect [2])

show ()

\section{A.2.6 Exemplo 6 - Dinâmica do modelo de Landau-Zener}

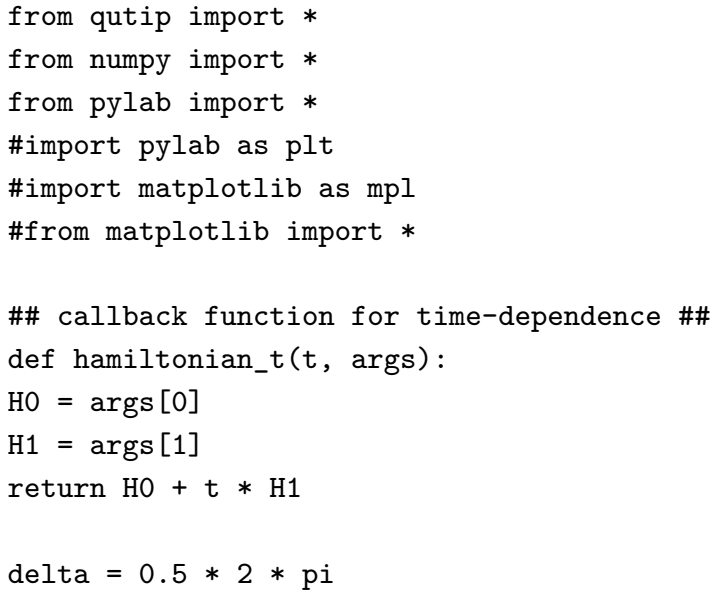


Figura 28 - Probabilidade do estado excitado
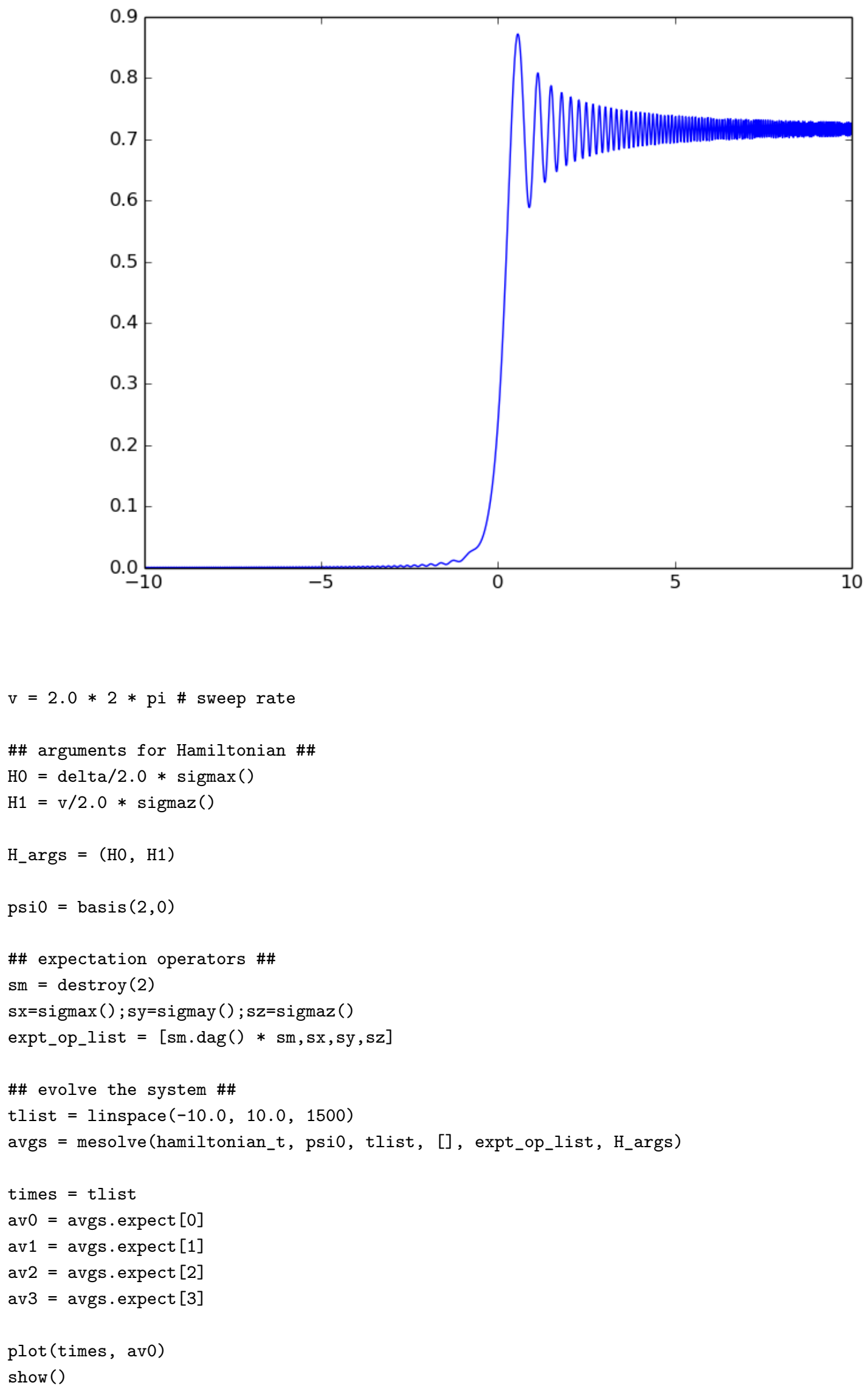


\section{A.2.7 Exemplo 7 - Representação na esfera de Bloch}

Figura 29 - Representação do modelo de Landau-Zener na esfera de Bloch

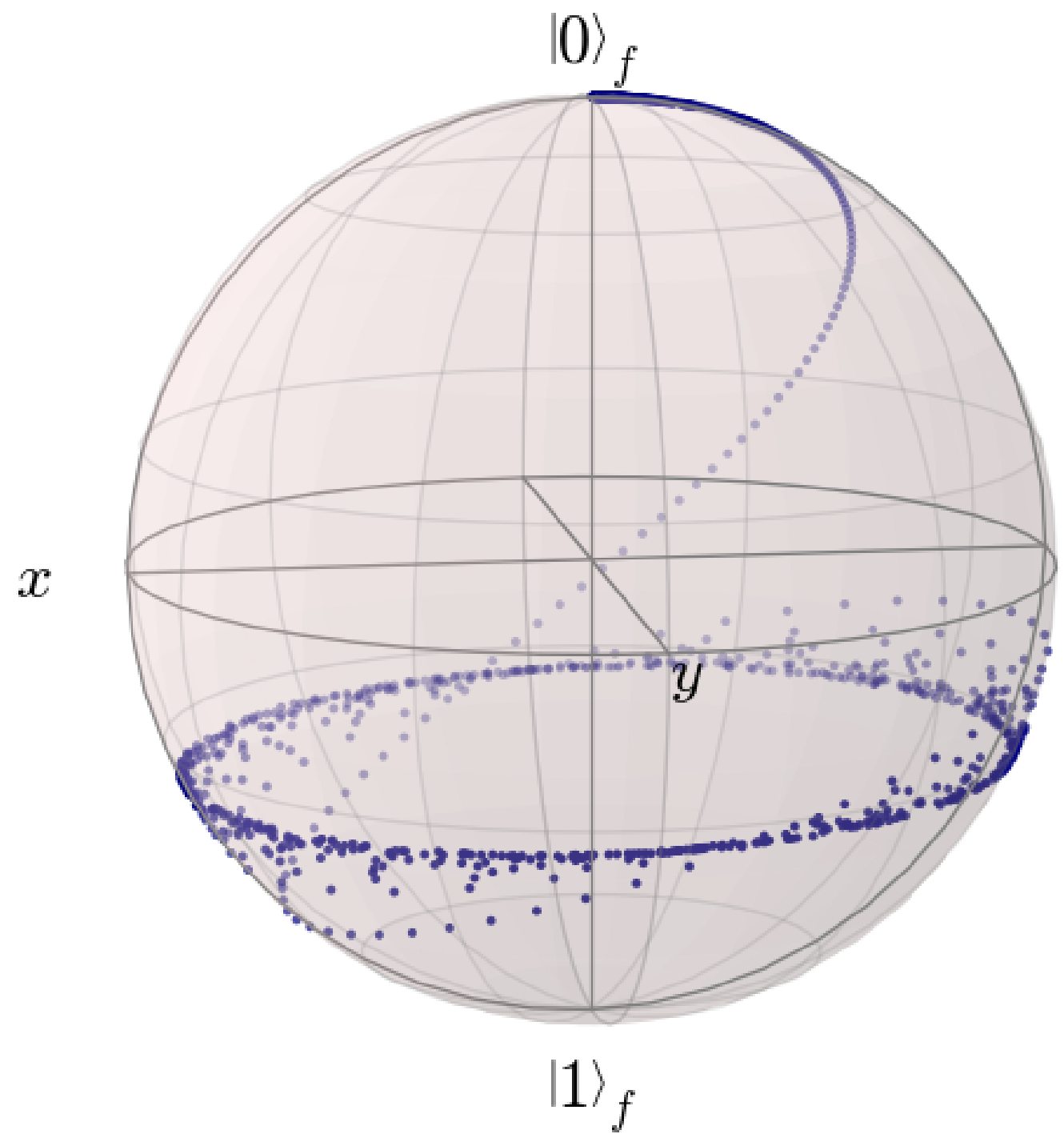

from qutip import *

from numpy import *

from pylab import *

import matplotlib as $\mathrm{mpl}$

from matplotlib import *

\#\# callback function for time-dependence \#\#

def hamiltonian_t( $t$, args):

$\mathrm{HO}=\operatorname{args}[0]$

$\mathrm{H} 1=\operatorname{args}[1]$

return $\mathrm{HO}+\mathrm{t} * \mathrm{H} 1$

delta $=0.5 * 2 * \mathrm{pi}$

$\mathrm{v}=2.0 * 2 * \mathrm{pi} \#$ sweep rate 


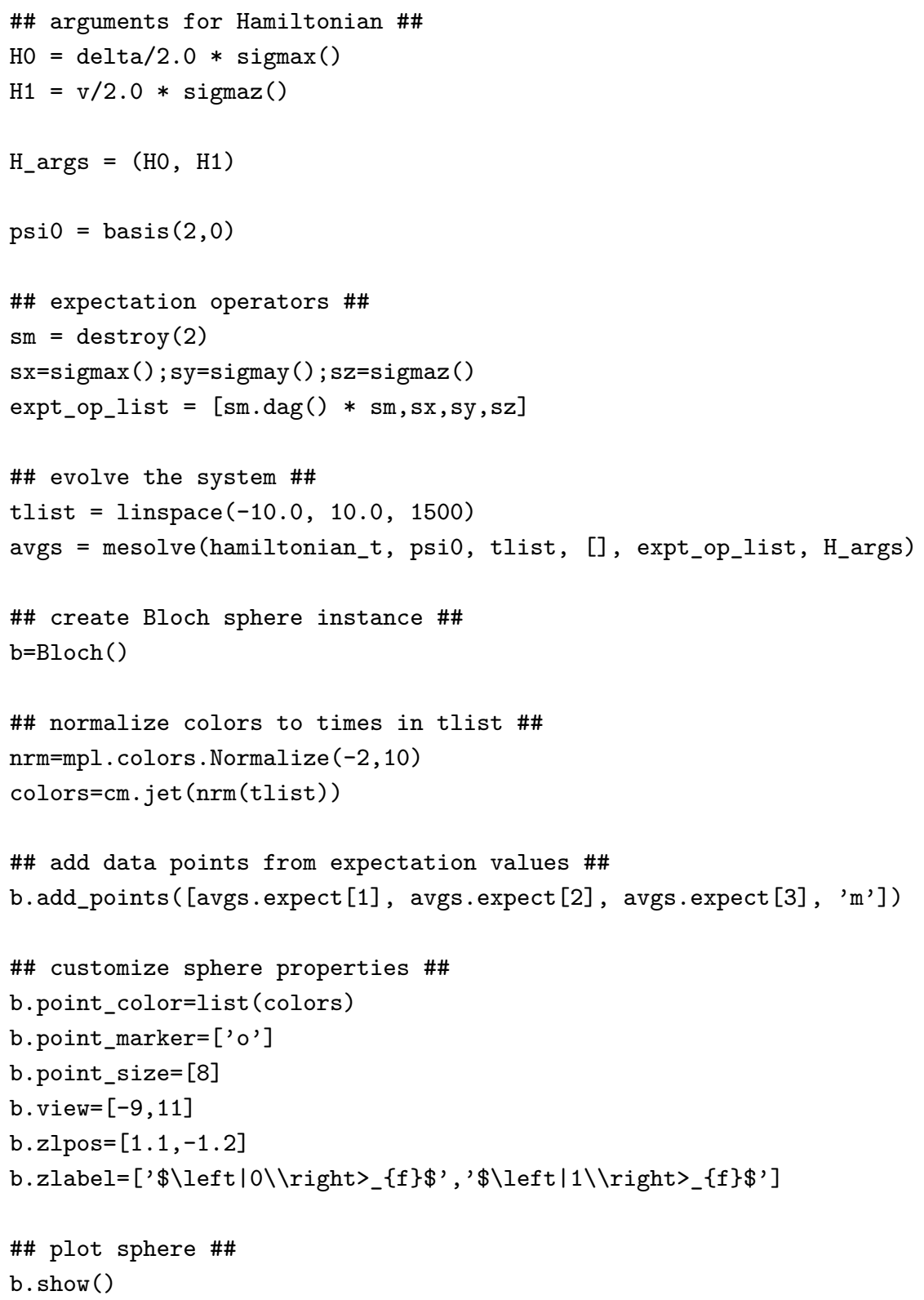

\section{A.3 Exemplos da versão 2}

\section{A.3.1 Exemplo 1 - Floquet}

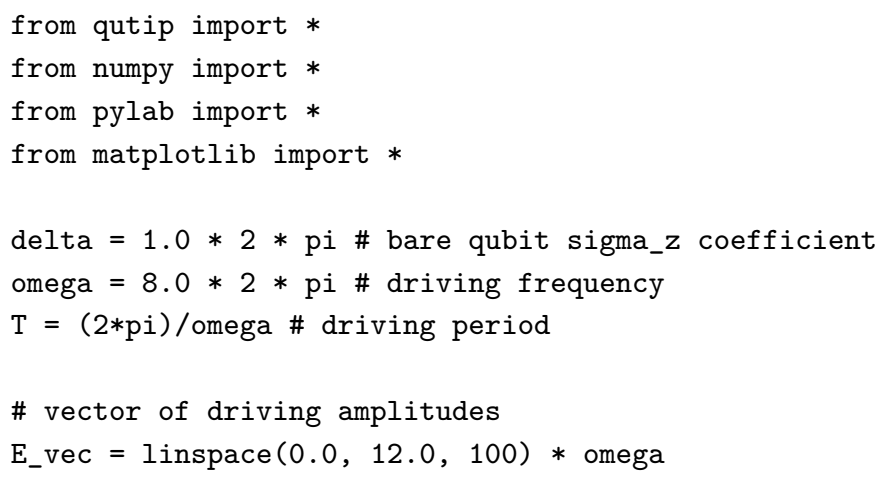


Figura 30 - Quasienergias da dinâmica do modelo de Floquet

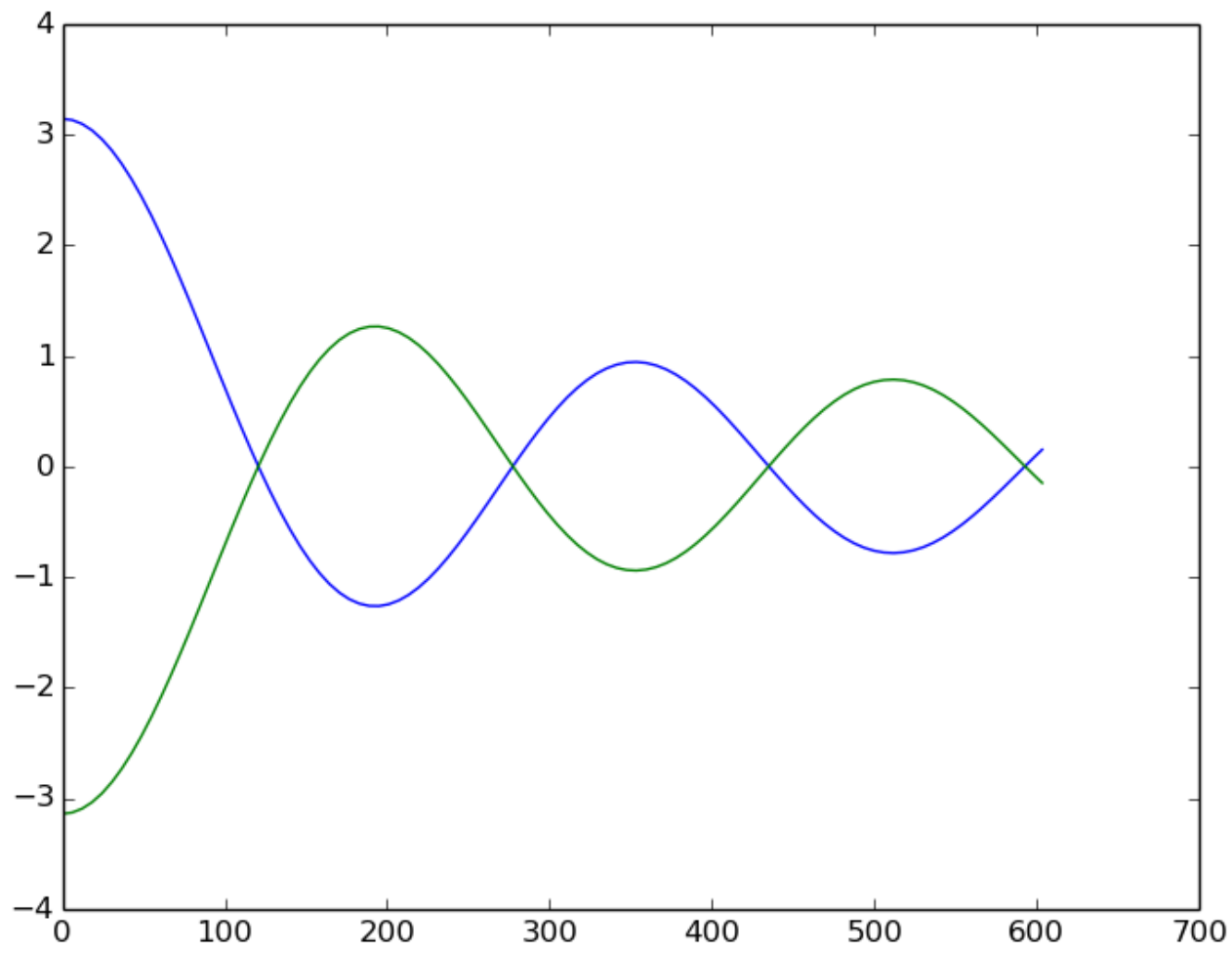

\# generate spin operators

$\mathrm{sx}=\operatorname{sigmax}()$

$\mathrm{sz}=\operatorname{sigmaz}()$

\# create array for storing energy values

q_energies $=\operatorname{zeros}\left(\left(\operatorname{len}\left(E_{-}\right.\right.\right.$vec $\left.\left.), 2\right)\right)$

\# define time-independent Hamiltonian term

$\mathrm{HO}=\operatorname{delta} / 2.0 * \mathrm{sz}$

$\operatorname{args}=\left\{{ }^{\prime} \mathrm{w}^{\prime}:\right.$ omega $\}$

plot (E_vec, q_energies)

show ()

\# loop over driving amplitudes

for idx, $E$ in enumerate(E_vec):

print $" i d x=\% d " \%$ idx

\# amplitude-dependent Hamiltonian term

$\mathrm{H} 1=\mathrm{E} / 2.0 * \mathrm{sx}$

$\# \mathrm{H}=\mathrm{HO}+\mathrm{H} 1 * \cos (\mathrm{w} * \mathrm{t})$ in 'list-string' format

$\mathrm{H}=\left[\mathrm{HO},\left[\mathrm{H} 1,{ }^{\prime} \cos (\mathrm{w} * \mathrm{t})^{\prime}\right]\right]$

\# find the Floquet modes

f_modes, f_energies $=$ floquet_modes $(\mathrm{H}, \mathrm{T}, \operatorname{args})$

\# record quasi-energies

q_energies $[i d x,:]=f_{-}$energies

plot (E_vec, q_energies) 
show()

\section{A.3.2 Exemplo 2 - Dinâmica Floquet}

Figura 31 - Dinâmica da equação de Floquet-Markov

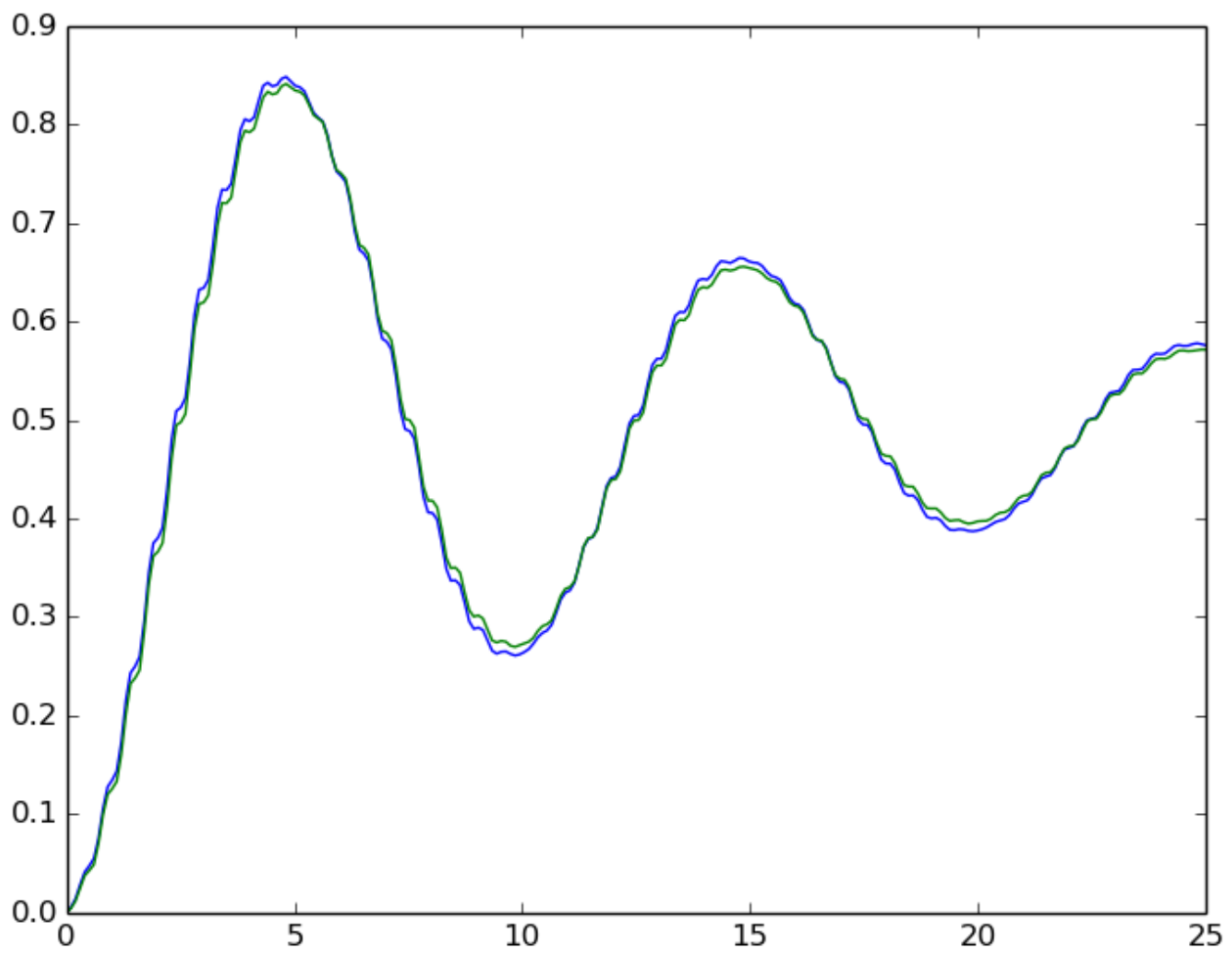

from qutip import *

from numpy import *

from pylab import *

gamma1 $=0.05 \#$ relaxation rate

gamma2 $=0.0 \#$ dephasing rate

delta $=0.0 * 2 *$ pi \# qubit sigma_x coefficient

eps0 $=1.0 * 2 *$ pi \# qubit sigma_z coefficient

$\mathrm{A}=0.1 * 2 *$ pi \# driving amplitude

$\mathrm{w}=1.0 * 2 * \mathrm{pi} \#$ driving frequency

$\mathrm{T}=2.0 * \mathrm{pi} / \mathrm{w} \#$ driving period

psi0 $=\operatorname{basis}(2,0) \#$ initial state

tlist $=$ linspace $(0,25.0,250)$

def J_cb(omega):

"" " Noise spectral density """

return $0.5 *$ gamma $1 *$ omega/ $(2 * \mathrm{pi})$

\# Hamiltonian in list-string format 


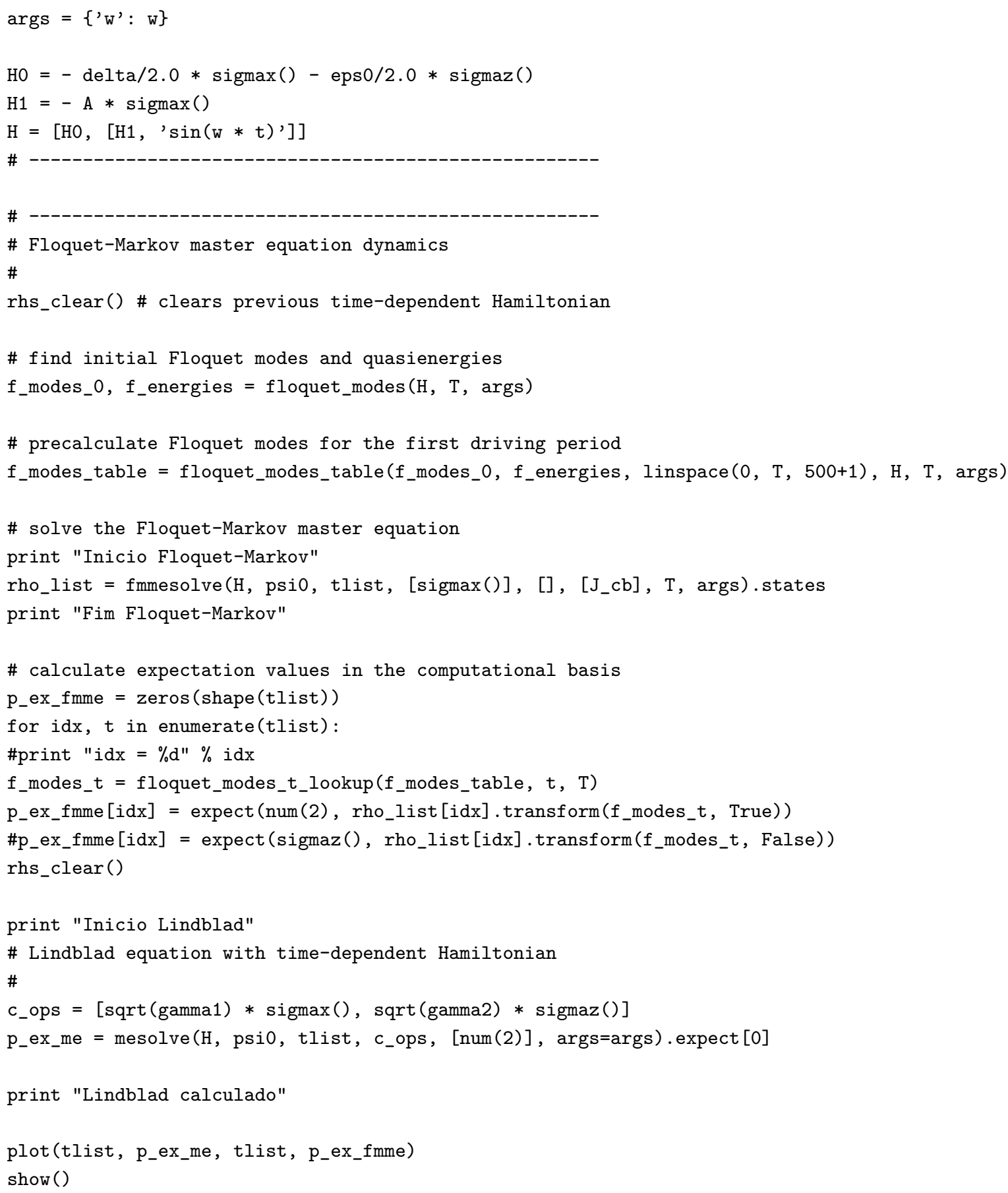

\section{A.3.3 Exemplo 3 - Equação de Bloch-Redfield}

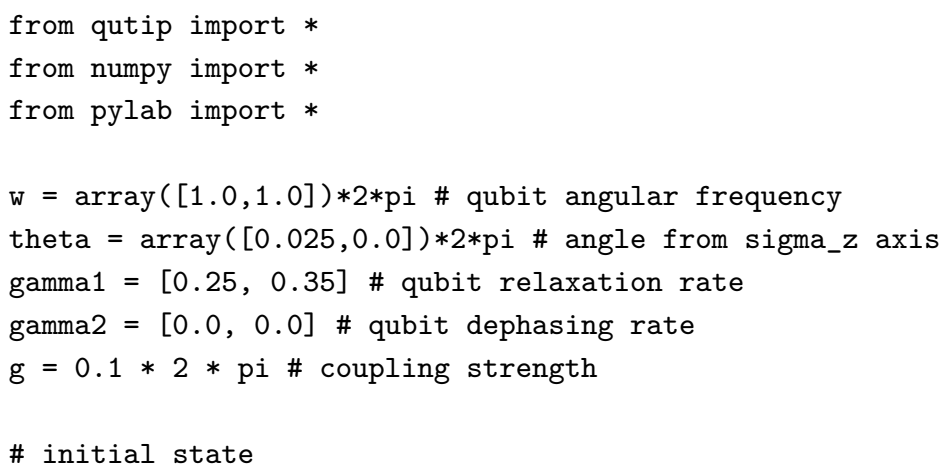


Figura 32 - Dinâmica da equação de Bloch-Redfield.
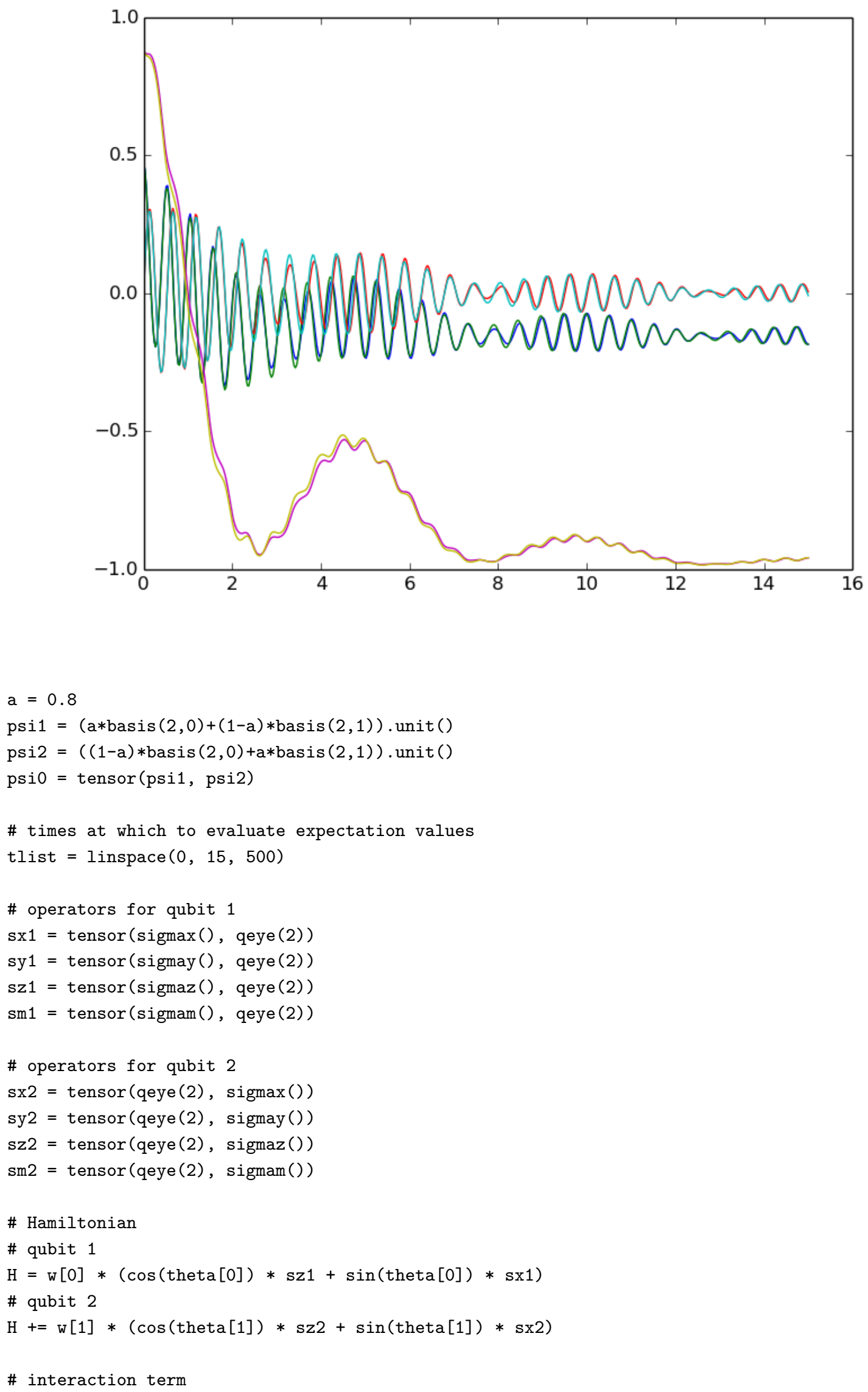


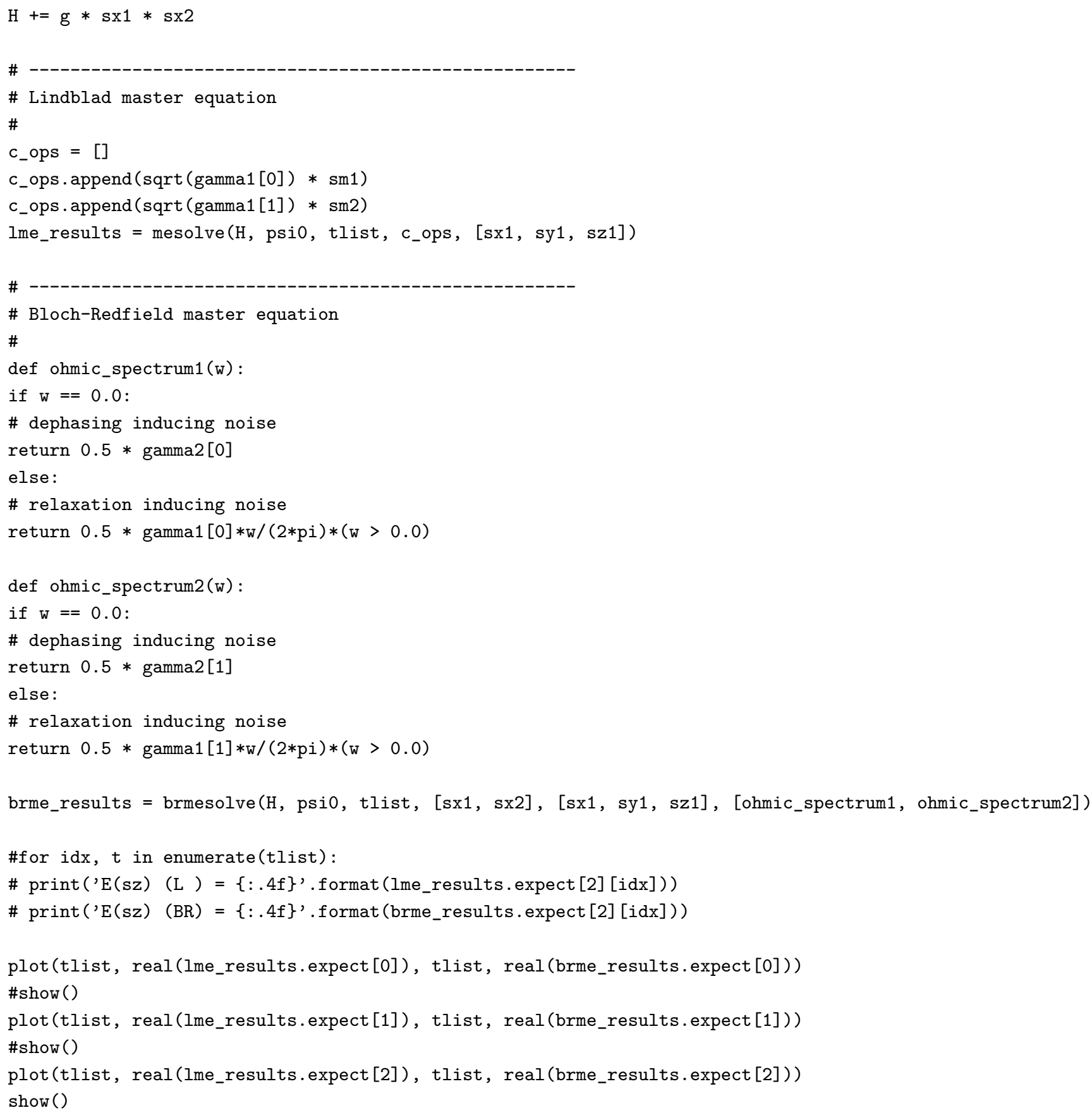

\section{A.3.4 Exemplo 5 - Quantum Process Tomography}

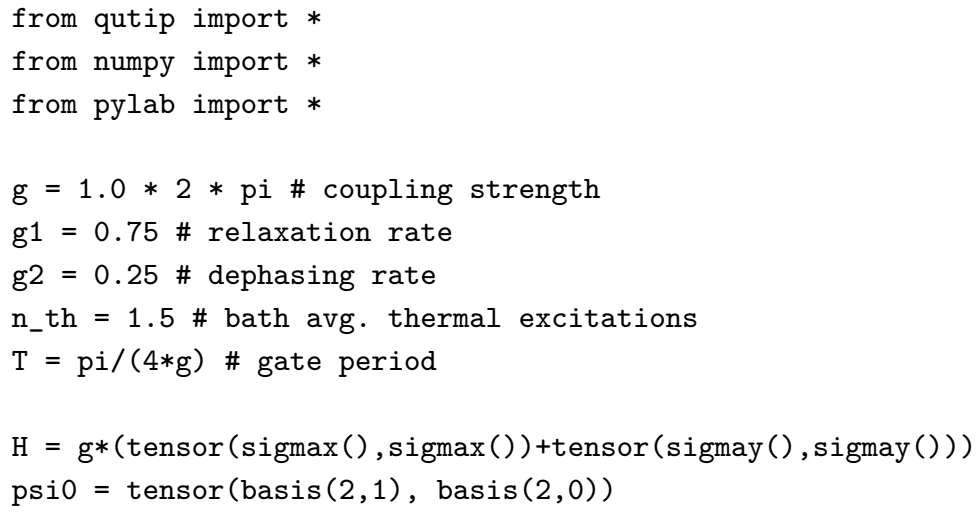


Figura 33 - QPT da dinâmica conservativa

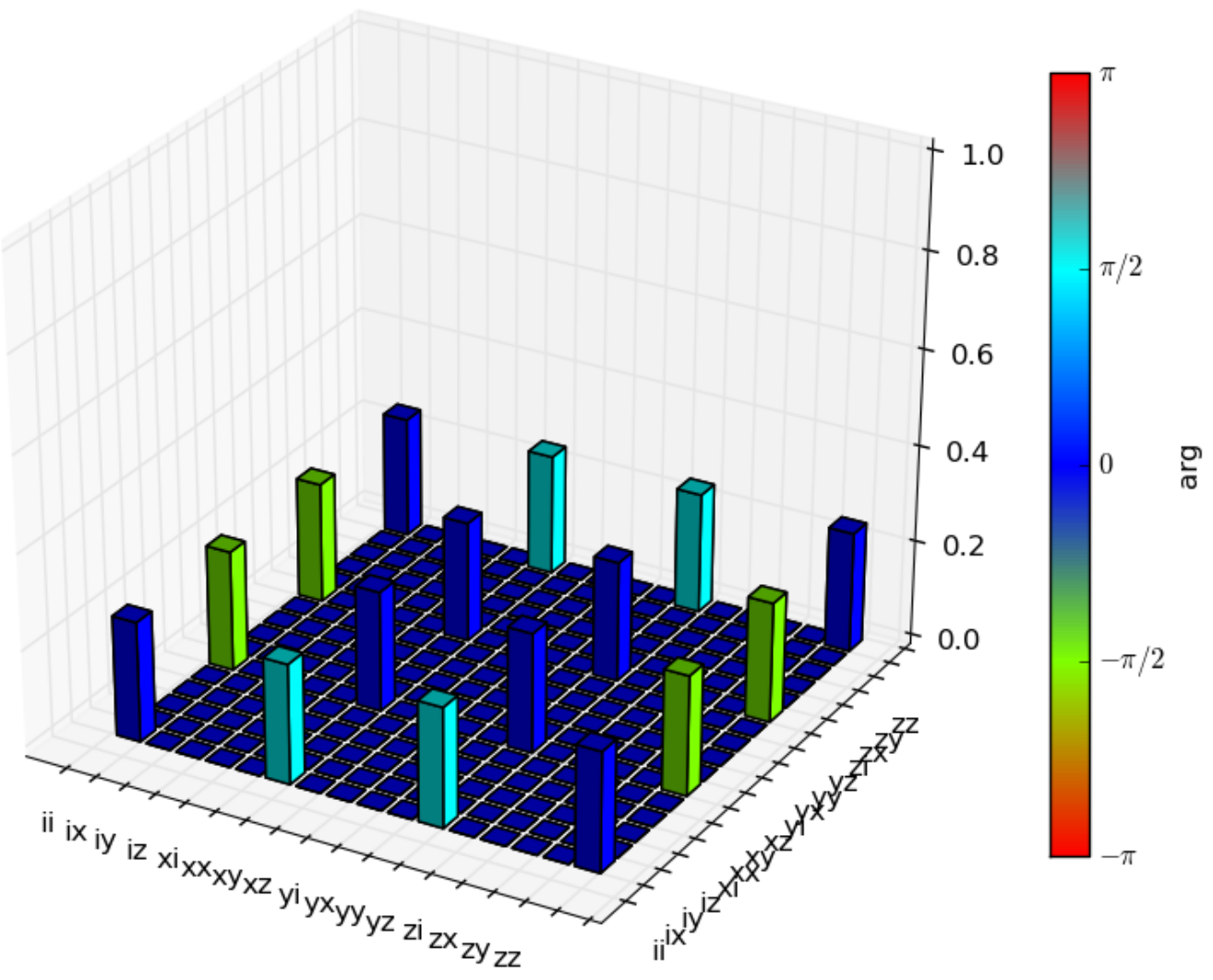

c_ops $=[]$

\# qubit 1 collapse operators

sm1 = tensor $(\operatorname{sigmam}()$, qeye $(2))$

sz1 $=\operatorname{tensor}(\operatorname{sigmaz}()$, qeye $(2))$

c_ops.append (sqrt (g1 * (1+n_th)) * sm1)

C_ops.append (sqrt (g1 * n_th) * sm1.dag ())

c_ops.append (sqrt (g2)* sz1)

\# qubit 2 collapse operators

sm2 = tensor (qeye(2), $\operatorname{sigmam}())$

sz2 $=$ tensor $($ qeye $(2), \operatorname{sigmaz}())$

c_ops.append (sqrt $\left.\left(\mathrm{g} 1 *\left(1+\mathrm{n}_{-} \mathrm{th}\right)\right) * \operatorname{sm} 2\right)$

C_ops.append (sqrt (g1 * n_th) * sm2.dag ())

c_ops .append (sqrt (g2) * sz2)

\# process tomography basis

op_basis $=[[q$ eye $(2), \operatorname{sigmax}(), \operatorname{sigmay}(), \operatorname{sigmaz}()]] * 2$

op_label $=[[" i ", " x "$, "y", "z"] $] * 2$

print "inicio"

\# dissipative gate

U_diss = propagator $\left(\mathrm{H}, \mathrm{T}, \mathrm{C}_{-} \mathrm{ops}\right)$

chi $=$ qpt (U_diss, op_basis)

qpt_plot_combined (chi, op_label)

show() 
Figura 34 - QPT da dinâmica dissipativa

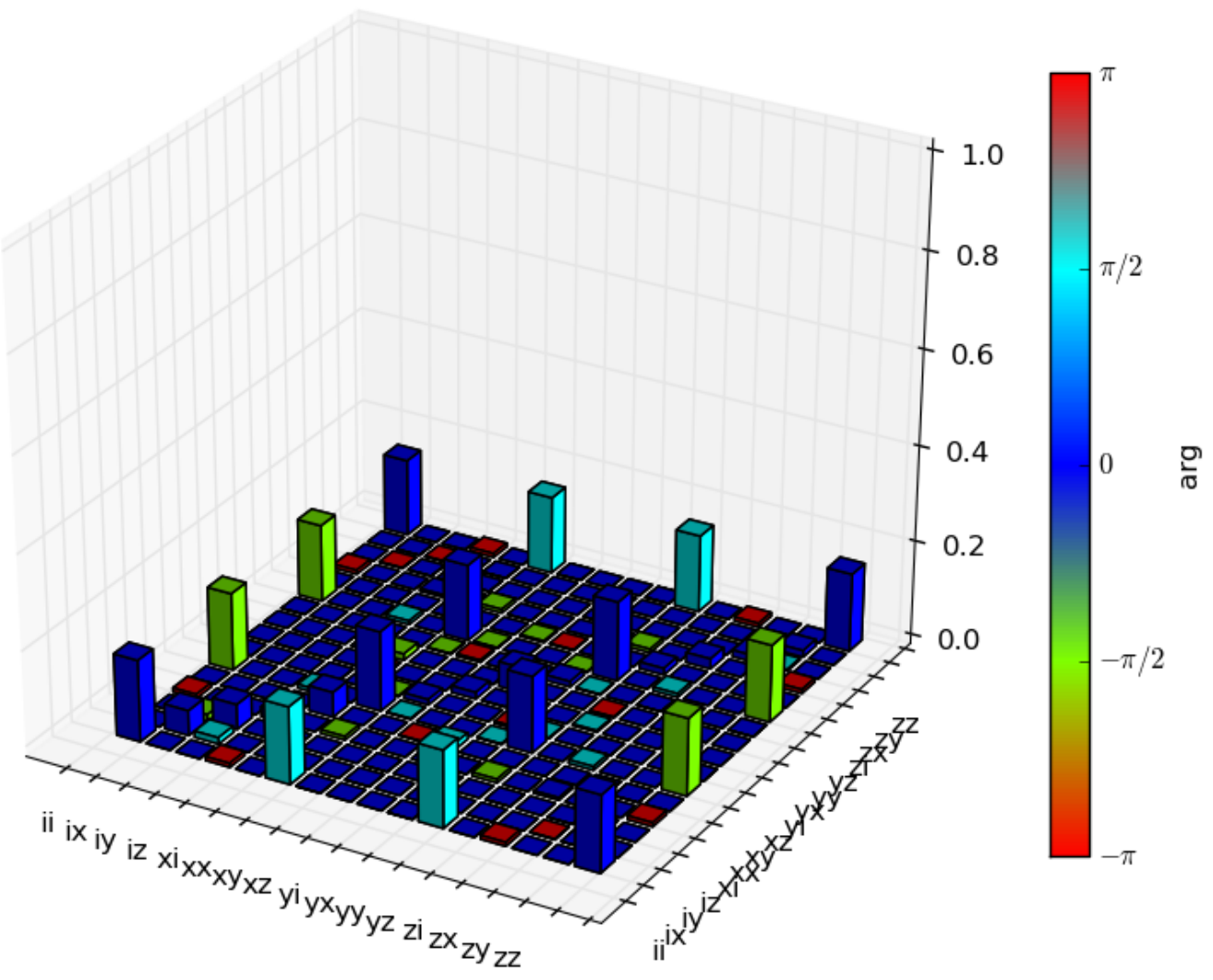

print "meio"

\# ideal gate

U_psi $=(-1 \mathrm{j} * \mathrm{H} * \mathrm{~T}) \cdot \operatorname{expm}()$

U_ideal $=\operatorname{spre}\left(U_{-}\right.$psi) $* \operatorname{spost}\left(U_{-}\right.$psi.dag ()$)$

chi $=$ qpt (U_ideal, op_basis)

qpt_plot_combined (chi, op_label)

show ()

print "fim"

\section{A.3.5 Exemplo 6 - Função de Wigner}

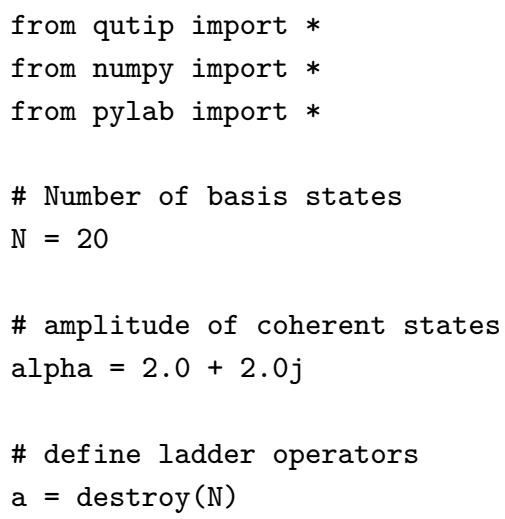


Figura 35 - Função de Wigner exemplificativa

\# define displacement operators

$\mathrm{D} 1$ = displace $(\mathrm{N}$, alpha $)$

$\mathrm{D} 2=\operatorname{displace}(\mathrm{N},-\mathrm{alpha})$

\# create superposition of coherent states + random ket

psi $=(\mathrm{D} 1+\mathrm{D} 2) * \operatorname{basis}(\mathrm{N}, 0)+0.5 * \operatorname{rand} \_k e t(\mathrm{~N})$

psi $=$ psi.unit () \# normalize

\# calculate Wigner function

$\mathrm{xvec}=\operatorname{linspace}(-5,5,500)$

yvec $=$ xvec

$\mathrm{W}=$ wigner (psi, $\mathrm{xvec}, \mathrm{yvec})$

\#\# new function calls \#\#

\# store Wigner function to file

file_data_store ("wigner.dat", W, numtype='real')

\# load input data from file

input_data $=f i l e \_d a t a \_r e a d($ 'wigner.dat')

\# plot using mayavi

from mayavi.mlab import * 
$\mathrm{X}, \mathrm{Y}=\operatorname{meshgrid}(\mathrm{xvec}, \mathrm{yvec})$

$\operatorname{surf}($ xvec, yvec, input_data, warp_scale='auto')

view $(0,45)$

show() 


\section{ANEXO B - Sistemas Dinâmicos}

Dado um espaço de fases, $M$, um processo evolutivo ou sistema dinâmico contínuo em $M$ é descrito pelo movimento de um ponto sobre uma curva no espaço de fases. A velocidade com que o processo evolui está definida para todos os pontos $x \in M$, ou seja, para todo $x$ está definido um vetor velocidade de fase. $O$ conjunto de vetores velocidade associados aos pontos de $M$ é chamado campo de vetores de velocidade de fase ou simplesmente campo de vetores.

Campo de Vetores: Seja $U$ um aberto de $\Re^{n}$, um campo de vetores de classe $C^{r}, r \geq 1$, em $U$ é um mapa $v: U \rightarrow \Re^{n}$ de classe $C^{r}$ que associa a cada ponto de $\mathrm{U}$ um vetor.

Existem casos em que o campo de vetores varia com o tempo. Neste caso, o campo de vetores é chamado não autônomo. Um campo de vetores não autônomo em $\Re^{n}$ é facilmente transformável em um campo de vetores autônomo (independente do tempo) em $\Re^{n+1}$. Assim não há perda de generalidade em restringir o estudo aos campos de vetores autônomos

Dado um campo de vetores $v$ em $U \subset \Re^{n}$, a equação diferencial ordinária definida por $v$ é dada por:

$$
\dot{x}=\frac{d x}{d t}=v(x)
$$

A solução de uma EDO é uma curva, $\phi: I \rightarrow U$, definida em um intervalo real e tomando valores no espaço de fases $U$, cuja derivada em todos os pontos é identica ao vetor associado pela EDO.

$$
\dot{\phi}(t)=\frac{d \phi}{d t}(t)=v(\phi(t))
$$

Se $x_{0}$ em $U$ é tal que $v\left(x_{0}\right)=0$ então $x_{0}$ é chamado ponto singular ou ponto de equilíbrio de $v$ e $\phi(t)=x_{0}$ é solução da EDO associada para todo $t \in \Re$. Se $v\left(x_{0}\right) \neq 0$ então $x_{0}$ é chamado ponto regular. Nem sempre EDO's possuem solução mas em condições particulares pode-se garanti-las.

A solução de equações diferenciais ordinárias: Seja $\dot{x}=v(x)$ uma EDO associada a um campo de vetores $v$ de classe $C^{k}$ definida para todos os pontos de um conjunto aberto, $U$, de $\Re^{n}$. Se $k \geq 1$ então, para todo ponto $x \in U$ existe $\epsilon_{x}>0$ e uma aplicação $\phi:\left(-\epsilon_{x}, \epsilon_{x}\right) \rightarrow U$ de classe $C^{k}$ tal que $\dot{\phi}(t)=\frac{d \phi}{d t}(t)=v(\phi(t))$ para todo 
$t \in\left(-\epsilon_{x}, \epsilon_{x}\right)$ e $\phi(0)=x$. Essa função $\phi$ também é única, ou seja, se $\gamma$ é uma função satisfazendo $\dot{\gamma}(t)=\frac{d \gamma}{d t}(t)=v(\gamma(t))$ então $\gamma=\phi$.

Como $\phi$ está definida para todo $x \in U$ pode-se estender a definição da função para $\phi: U \times\left(-\epsilon_{x}, \epsilon_{x}\right) \rightarrow U$ tal que $\phi\left(x_{0}, 0\right)=x_{0}$ e $\phi\left(x_{0}, t\right)=\phi_{x_{0}}(t)$ é solução da EDO. Esta extensão coloca em evidência a dependência que a solução de uma EDO possui com relação às condições iniciais. ${ }^{1}$

Nem sempre a solução de uma EDO está definida para todo $t \in \Re$ mas a seguinte proposição é válida:

Solução maximal: Seja $v$ um campo vetorial de classe $C^{k}, k \geq 1$ em $U$. Para todo $x \in U$ existem números reais $\omega_{+}\left(x_{0}\right)$ e $\omega_{-}\left(x_{0}\right)$ tais que:

$$
\phi_{x_{0}}:\left(\omega_{-}\left(x_{0}\right), \omega_{+}\left(x_{0}\right)\right) \rightarrow U
$$

é solução da EDO associada a $v$ e qualquer outra $\gamma: I \rightarrow U$ solução da EDO é tal que $I \subset\left(\omega_{-}\left(x_{0}\right), \omega_{+}\left(x_{0}\right)\right) . \quad \phi_{x_{0}}$, assim definida, é chamada solução maximal da EDO com condição inicial $x_{0}$.

Frequentemente $\omega_{+}\left(x_{0}\right)<+\infty$ e $\omega_{-}\left(x_{0}\right)>-\infty$. Nesses casos pode-se mostrar que $\phi_{x_{0}}(t) \rightarrow \partial U$ ou $\phi_{x_{0}}(t) \rightarrow \infty$ ou ambos para $t \rightarrow \omega_{+}\left(x_{0}\right)$ e $t \rightarrow \omega_{-}\left(x_{0}\right)$. Onde $\partial U$ é a borda de $U$.

Se $v$ éum campo vetorial de classe $C^{k}, k \geq 1$ em $\Re^{n}$ e existe $M>0$ tal que $M>\|v(x)\|$ para todo $x \in \Re^{n}$ então $\phi\left(x_{0}, t\right)=\phi_{x_{0}}(t)$ solução da EDO associada está definida para todo $t \in \Re$.

Similarmente, se $v$ é um campo vetorial de classe $C^{k}, k \geq 1$ em $M$, uma variedade diferenciável compacta (por exemplo uma esfera ou um toro), então $\phi\left(x_{0}, t\right)=\phi_{x_{0}}(t)$ solução da EDO associada está definida para todo $t \in \Re$.

Seja $\phi: I \rightarrow U$ a solução de uma EDO no espaço de fase $U$. A imagem de $\phi$ em $U$ é chamada uma órbita da EDO em $U$ e o gráfico de $\phi$ contido em $\Re \times U$ é chamado curva integral. A decomposição de $U$ nas órbitas de uma EDO definida em $U$ é chamada retrato de fase da EDO. As órbitas são orientadas no sentido positivo de $t$.

Tipos de Órbitas: Seja $v$ um campo vetorial de classe $C^{k}, k \geq 1$ em $U$ e $\phi\left(x_{0}, t\right)=\phi_{x_{0}}(t)$ solução da EDO associada. Se $\phi_{x_{0}}: I \rightarrow U$ é solução maximal então verifica-se uma única:

1. $\phi_{x_{0}}$ é injetora.

2. $I=\Re$ e $\phi_{x_{0}}=x_{0}$. Em pontos singulares.

$\overline{1}$ O problema de se encontrar $x=\phi(t)$ tal que $\dot{x}=v(x)$ e $\phi\left(t_{0}\right)=x_{0}$ problema de Cauchy. 
3. $I=\Re$ e $\phi_{x_{0}}$ é uma órbita periódica, ou seja, $\phi_{x_{0}}(\tau)=\phi_{x_{0}}(0)=x_{0}$ para algum $\tau>0$ chamado período da órbita.

Homeomorfismo: Sejam $U$ e $V$ abertos de $\Re^{n}$. Um homeomorfismo entre $U$ e $V$ é uma aplicação $h: U \rightarrow V$ tal que $h$ é bijetora, ou seja, possui uma inversa $h^{-1}$ e tanto $h$ quanto $h^{-1}$ são contínuas. Pode-se pensar em um homeomorfismo como sendo uma mudança de coordenadas que conserva certas propriedades entre conjuntos e funções de dois abertos. ${ }^{2}$ Por exemplo se $f: U \rightarrow \Re$ é uma função contínua então $g=f \circ h^{-1}: V \rightarrow \Re$ também é contínua.

Difeomorfismo: Sejam $U$ e $V$ abertos de $\Re^{n}$. Um difeomorfismo de classe $C^{k}$ entre $U$ e $V$ é um homeomorfismo $h: U \rightarrow V$ tal que $h$ possui $k$ derivadas em todo ponto $x \in U$ e $h^{-1}$ possui $k$ derivadas em todo ponto $y \in V$. A derivada de $\mathrm{f}$ em um ponto x é uma matriz

$$
d f(x)=a_{i j}=\frac{\partial f_{i}}{\partial x_{j}}(x), d f(x) \cdot v=a_{i j} v_{j}=\frac{\partial f_{i}}{\partial x_{j}}(x) v_{j}
$$

e seu determinante, chamado jacobiano, é diferente de 0 . Reciprocamente, se o jacobiano de uma função $f: U \rightarrow V$ é diferente de 0 em um ponto $x$ então $f$ é localmente um difeomorfismo, ou seja, existe um aberto $U_{0} \subset U$ com $x \in U_{0}$ tal que $f: U_{0} \rightarrow f\left(U_{0}\right)$ é um difeomorfismo. ${ }^{3}$

Um grupo é um conjunto, $G$, no qual está definida uma operação binária, $s$ : $G \times G \rightarrow G$ (por exemplo soma ou multiplicação) de tal forma que:

1. Para quaisquer elementos $x$ e $y, s(x, y) \in G$ e $s(y, x) \in G$.

2. Existe $e \in G$ tal que $s(x, e)=s(e, x)=x$ onde $e$ é o elemento neutro.

3. Para todo $x \in G$ existe $x^{-1} \in G$ tal que $s\left(x^{-1}, x\right)=s\left(x, x^{-1}\right) \in G$.

Os números reais, $\Re$, juntamente com a operação de soma formam um grupo tal como definido acima. Refere-se a ele como o grupo $(\Re,+)$

Grupos de Difeomorfismos: Um grupo de difeomorfismos a um parâmetro de classe $C^{k}, k \geq 1,\left\{g^{t}\right\}$, de um domínio $U$ é uma aplicação $g: U \times \Re \rightarrow U, g(x, t)=g^{t} x$ tal que:

1. $g$ é de classe $C^{k}, k \geq 1$.

2. Para cada $t \in \Re, g^{t}: U \rightarrow U$ é um difeomorfismo.

2 Um homeomorfismo conserva as propriedades topológicas de um espaço ou um subconjunto deste espaço. Aberto, Fechado, Conexo, Compacto são exemplos de propriedades topológicas de um conjunto.

3 Este é o enunciado de um importante resultado de análise chamado teorema da função inversa. 
3. $g$ satisfaz a propriedade de grupo dada por $g^{t} \circ g^{s}=g^{t+s}$ para todo tes $\in \Re$ e $g^{0}=I d$ ou seja, $g^{0} x=x$.

Note-se que 1 e 3 implicam 2. Verificar os livros de Arnold (ANOSOV et al., 1988) e (ARNOLD, 1978) para detalhes a respeito de grupos de difeomorfismos.

A terminologia grupo de difeomorfismos quer dizer que existe um conjunto de difeomorfismos cujos elementos estão correspondência biunívoca com os elementos de um grupo (propriedades 1 e 2). E mais, é necessário que a correspondência respeite a operação do grupo (propriedade 3). O grupo, $G$, representa o espaço em está definido o tempo, a variável independente que evolui.

Sistema Dinâmico: Dado um grupo, $(G,+)$, e um espaço de fases, $S$, um sistema dinâmico é dado por uma aplicação $\Phi: G \times S \rightarrow S$ onde, escrevendo-se $\Phi^{t}(x)=\Phi(t, x)$, o mapa $\Phi^{t}: S \rightarrow S$ satisfaz:

1. $\Phi^{0}: S \rightarrow S$ é a apliacação identidade e 0 é o elemento neutro do grupo

2. $\Phi^{t} \circ \Phi^{s}=\Phi^{t+s}$

Com essa definição genérica de sistema dinâmico estamos englobando pelo menos dois tipos de sistemas. Os sistemas discretos, quando $G$ é o grupo $(Z,+)$ dos números inteiros, e os sistemas contínuos quando $G$ é o grupo $(\Re,+)$ dos números reais ${ }^{4}$. Quando o sistema dinâmico é contínuo, podemos exigir que o mapa $(t, x) \mapsto \Phi^{t} x$ seja um difeomorfismo. Neste caso tem-se um sistema dinâmico diferenciável que é equivalente a um grupo de difeomorfismos. Quando a aplicação $(t, x) \mapsto \Phi^{t} x$ é apenas um homeomorfismo tem-se um sistema dinâmico topológico. Sistemas dinâmicos contínuos são também chamados fluxos. No caso de sistemas dinâmicos discretos analisamos apenas se $\Phi=\Phi^{1}$ é um homeomorfismo ou difeomorfismo do espaço de fases $S$. Os sistemas dinâmicos discretos são também chamados mapas ou cascatas. Ver definição e desenvolvimento em Anosov, (ANOSOV et al., 1988).

Seja $S \subset \Re^{n}$, aberto, o espaço de fases de uma EDO e $\phi(x, t)=\phi^{t} x$ a solução maximal da EDO definida em $J(x) \subset \Re$ tal que $\phi^{0} x=x$ para todo $x \in S$. Define-se $\Omega \subset S \times \Re$, aberto, como $\Omega=\{(x, t) \in S \times \Re: t \in J(x)\}$. A aplicação $\phi: \Omega \rightarrow S$ onde $\phi^{t} x$ é a solução maximal da EDO passando por $x$ é chamada o fluxo da EDO. Frequentemente chama-se de fluxo o grupo de difeomorfismos $\left\{\Phi^{t}\right\}$. Não há diferença fundamental entre as duas formas uma vez que são duas formas diferentes de se referir ao conjunto de soluções de uma EDO. Por conveniência aplica-se uma ou outra definição.

$4 \quad$ No caso de grupos finitos, os sistemas dinâmicos definidos são capazes de representar autômatos finitos, estruturas estudadas em lógica e teoria da computação e que estão relacionadas à definição da máquina de Turing como dispositivo fundamental da computação clássica. 
O campo de vetores de velocidade de fase de um sistema dinâmico diferenciável $\left\{g^{t}\right\}$ é dado por

$$
v(x)=\left.\frac{d g^{t}(x)}{d t}\right|_{t=0}
$$

Fluxos e Sistemas Dinâmicos Diferenciáveis: Se se fixar um valor $x_{0} \in U$ para um sistema dinâmico diferenciável $g: U \times \Re \rightarrow U$ temos que a aplicação $\phi: \Re \rightarrow U$ dada por $\phi(t)=g^{t} x_{0}$ é solução da equação diferencial associada ao campo de vetores de velocidade de fase de $\left\{g^{t}\right\}$. O sistema dinâmico diferenciável $\left\{g^{t}\right\}$ é o fluxo da EDO

$$
\dot{x}=v(x)=\left.\frac{d g^{t}(x)}{d t}\right|_{t=0}
$$

$\mathrm{Na}$ realidade, todo sistema dinâmico diferenciável é um fluxo e vice-versa. Todo sistema dinâmico diferenciável está associado a uma EDO como mostrado acima. Em determinadas condições a recíproca é também verdadeira, ou seja, as soluções maximais de uma EDO definem uma estrutura parecida com um fluxo. Se as EDO's tiverem soluções globais, para todo $t \in \Re$, então as soluções $\phi(x, t)=\phi^{t} x$ são um sistema dinâmico diferenciável no espaço de fases da EDO. Se as soluções não forem globais o espaço onde está definida a variável temporal deixa de ser um grupo porque o estado do sistema deixa de estar definido para todo $t \in \Re$.

Campos de Vetores Parametrizados: Seja $W$ um aberto de $\Re^{p}$ e $U$ um aberto de $\Re^{n}$, um campo de vetores de classe $C^{r}$ em $U, r \geq 1$ parametrizado em $W$ é uma aplicação de classe $C^{r}$ em $U, r \geq 1, v: U \times W \rightarrow \Re^{n}$ tal que para todo $\lambda \in \Re^{p}$, fixo, $v_{\lambda}=v(\cdot, \lambda)$ é um campo de vetores de classe $C^{r}$ em $U, r \geq 1$.

A solução da $\operatorname{EDO} \dot{x}=v_{\lambda}(x)=v(x, \lambda)$ para cada $\lambda \in \Re^{p}$, fixo, é indicada por $\phi(t, x, \lambda)=\phi_{\lambda}^{t}(x)$.

Teorema de Diferenciabilidade: Seja $\dot{x}=v_{\lambda}(x)$ uma EDO associada a um campo de vetores parametrizado, $v$, de classe $C^{k}, k \geq 1$, definida para todos os pontos de um conjunto aberto, $U$, de $\Re^{n}$. O fluxo da $\operatorname{EDO}, \phi(t, x, \lambda)=\phi_{\lambda}^{t}(x)$ é de classe $C^{k}$.

O significado do teorema acima é que a solução da EDO é diferenciável com relação à condição inicial e aos parâmetros e pode ser aproximada localmente por suas derivadas.

No estudo qualitativo de Sistemas Dinâmicos é importante definir propriedades características e separar os sistemas em grupos. Certos difeomorfismos, ou homeomorfismos, entre espaços de fase são capazes de estabelecer uma relação de equivalência entre Sistemas Dinâmicos.

Ação de Difeomorfismos em Campos de Vetores: Sejam $h: M \rightarrow N$ um difeomorfismo e $v: M \rightarrow \Re^{n}$ um campo de vetores no espaço de fases $M$. Nessas condições pode-se definir em $N$ um campo de vetores, $w$, chamado push-forward de $v$ por 
$h$, indicado por $w=h_{*} v$, onde, para cada $x \in M$,

$$
w(y)=h_{* x} v(x): h_{* x}=d h(x)=\frac{\partial h}{\partial x}(x), y=h(x)
$$

em outras palavras, $h$ leva $x$ em $y=h(x)$ e o vetor $v(x)$ em $w(x)=d h(x) \cdot v(x)=$ $\frac{\partial h}{\partial x}(x) v(x)$. A derivada de $h$ em $x, h_{* x}=d h(x)=\frac{\partial h}{\partial x}(x)$, é um operador linear para todo $x \in M$. Como $h$ é um difeomorfismo é garantido que este operador linear é um isomorfismo ${ }^{5}$ em $\Re^{n}$ e portanto $w(y)=0 \Leftrightarrow v(x)=0$.

Sistemas de Coordenadas: Seja $M$ um conjunto. Um sistema de coordenadas em $M$ é uma função bijetora $f: U \rightarrow \tilde{U}$ onde $U \subset M$ e $\tilde{U}$ é um aberto de $\Re^{n}$.

Mudança de Coordenadas: Sejam $f: U \rightarrow \tilde{U}$ e $g: V \rightarrow \tilde{V}$ sistemas de coordenadas em $M$ com $U \cap V \neq \oslash$. A mudança de coordenadas, $f \rightarrow g$, definida em $f(U \cap V)$ é dada por $g \circ f^{-1}: f(U \cap V) \rightarrow g(U \cap V)$. Um difeomorfismo de classe $C^{k}$ é uma mudança de coordenadas de classe $C^{k}$ e um homeomorfismo é uma mudaça de coordenadas de de classe $C^{0}$.

Em $\Re^{n}$ os sistemas de coordenadas ortonormais respeitam a definição acima. Denota-se por $\left(p_{1}, \cdots, p_{n}\right)_{x}$ as coordenadas de $p \in \Re^{n}$ no sistema de coordenadas $x$. Omite-se o subscrito quando não há possibilidade de confusão.

Dado um sistema de coordenadas $x$, frequentemente é usada a notação $\frac{\partial}{\partial x_{i}}$ para designar o vetor unitário na direção $x_{i}$. Um vetor genérico é indicado por

$$
v=v_{1} \frac{\partial}{\partial x_{1}}+\cdots+v_{n} \frac{\partial}{\partial x_{n}}
$$

Isto significa atribuir a um vetor o significado de operador de diferenciação. Por exemplo, em $\Re^{1}$ se $f: U \rightarrow \Re$ é uma função diferenciável então

$$
v(f)=v_{1} \frac{\partial}{\partial x_{1}}(f)=v_{1} \frac{\partial f}{\partial x_{1}}
$$

Usando a notação acima, pode-se calcular a ação de um difeomorfismo em um campo de vetores de forma bastante natural como, por exemplo em $R^{1}$ :

$$
\frac{\partial}{\partial y}=\frac{1}{g} \frac{\partial}{\partial x}, y=g(x)
$$

Equivalência: Dois sistemas dinâmicos $\phi$ e $\psi$ são topologicamente (resp. $C^{k}$ equivalentes) se existe um homeomorfismo (resp. difeomorfismo de classe $C^{k}$ ) tal que

$$
g \circ \phi_{x}=\psi_{g(x)}
$$

$\overline{5}$ Um operador linear, $A: \Re^{n} \rightarrow \Re^{n}$, é dito um isomorfismo quando é uma aplicação injetora e sobrejetora o que garante que o kernel de $A$ e $A^{-1}$ é $\{\overrightarrow{0}\}$. O kernel de um operador linear é dado por $\operatorname{ker}(A)=\left\{\vec{v} \in \Re^{n}: A(\vec{v})=\overrightarrow{0}\right\}$. 
em outras palavras, $g$ leva órbitas de $\phi$ em órbitas de $\psi$ preservando a orientação temporal. Um homeomorfismo, ou difeomorfismo, $g: S \rightarrow T$ age no fluxo de fase, $\phi^{t}: S \rightarrow S$, de uma EDO como:

$$
\psi^{t}=g \circ \phi^{t} \circ g^{-1}
$$

pode-se considerar $g$ como uma mudança de coordenadas e pensar no fluxo $\psi^{t}$ como o fluxo $\phi$ escrito em novas coordenadas.

Mudança de Coordenadas em uma EDO: Sejam $g: M \rightarrow N$ uma mudança de coordenadas de classe $C^{k}, k \geq 1, v$ um campo de vetores em $M$ e $w=g_{*} v$ o pushforward de $v$ por $g$. Os fluxos de fase $\phi^{t}$ e $\psi^{t}$ gerados respectivamente por $v$ e $w$ em $M$ e $N$ são $C^{k}$ equivalentes, ou seja, se relacionam por $\psi^{t}=g \circ \phi^{t} \circ g^{-1}$.

Seção Transversal: Sejam $v: U \rightarrow \Re^{n}$ um campo vetorial de classe $C^{k}, k \geq 1$, e $f: A \rightarrow U$ uma função de classe $C^{k}, k \geq 1$, definida em $A$, um aberto de $\Re^{n-1}$. O conjunto $\Sigma=f(A)$ é chamado uma seção transversal local do campo vetorial $v$ se, para todo $a \in A,\left\{v(f(a)) \oplus d f(a)\left(\Re^{n-1}\right)\right\}=\Re^{n}$, ou seja, o campo vetorial nunca é tangente à superfície $\Sigma$ definida por $f$. Para cada ponto $x \in \Sigma$ existe uma base de $\Re^{n}$ formada por $v(x)$ e vetores pertencentes ao plano tangente a $\Sigma$ em $x$. Note-se que se $\Sigma$ é uma seção transversal local de $v$ então $v(x) \neq 0$ para todo $x \in \Sigma$

Teorema do Fluxo Tubular: Seja $x \in U$ um ponto não singular do campo vetorial de classe $C^{k}, k \geq 1, v: U \rightarrow \Re^{n}$ e $f: A \rightarrow \Sigma$ uma seção transvesal local de $v$ de classe $C^{r}, r \geq 1, \operatorname{com} f(0)=x$. Então existe uma vizinhança aberta, $\tilde{U}$, de $x$ em $U$ e um difeomorfismo $h: \tilde{U} \rightarrow(-\epsilon, \epsilon) \times B$ de classe $C^{l}, l \leq$ inf $\{k, r\}$, onde $\epsilon \in \Re$ é positivo e $B$ é uma bola aberta em $A$ contendo o 0 tais que:

1. $h(\Sigma \cap \tilde{U})=\{0\} \times B$

2. $h$ é uma equivalência de classe $C^{l}$ entre o campo $v: \tilde{U} \rightarrow \Re^{n}$ e o campo constante $w:(-\epsilon, \epsilon) \times B \rightarrow \Re^{n}$ dado por $w=(1,0, \cdots, 0) \in \Re^{n}$.

O principal significado do teorema acima é que em uma vizinhança de um um ponto regular de um campo vetorial existe um difeomorfismo que "retifica"o campo de vetores. Note-se que esse é um teorema local. Como consequência, tem-se que dados dois campos de vetores em $\Re^{n}$ sempre existe uma mudança de coordenadas que leva um campo ao outro em uma vizinhança em um ponto regular qualquer. Ideias a acrescentar no texto principal 


\section{ANEXO C - Código fonte em MATLAB}

Apresentamos a seguir o código-fonte utilizado para simular as equações apresentadas no Capítulo 4, referentes ao modelo apresentado em (DOHERTY et al., 2000). Utilizamos na simulação versão 2015b.

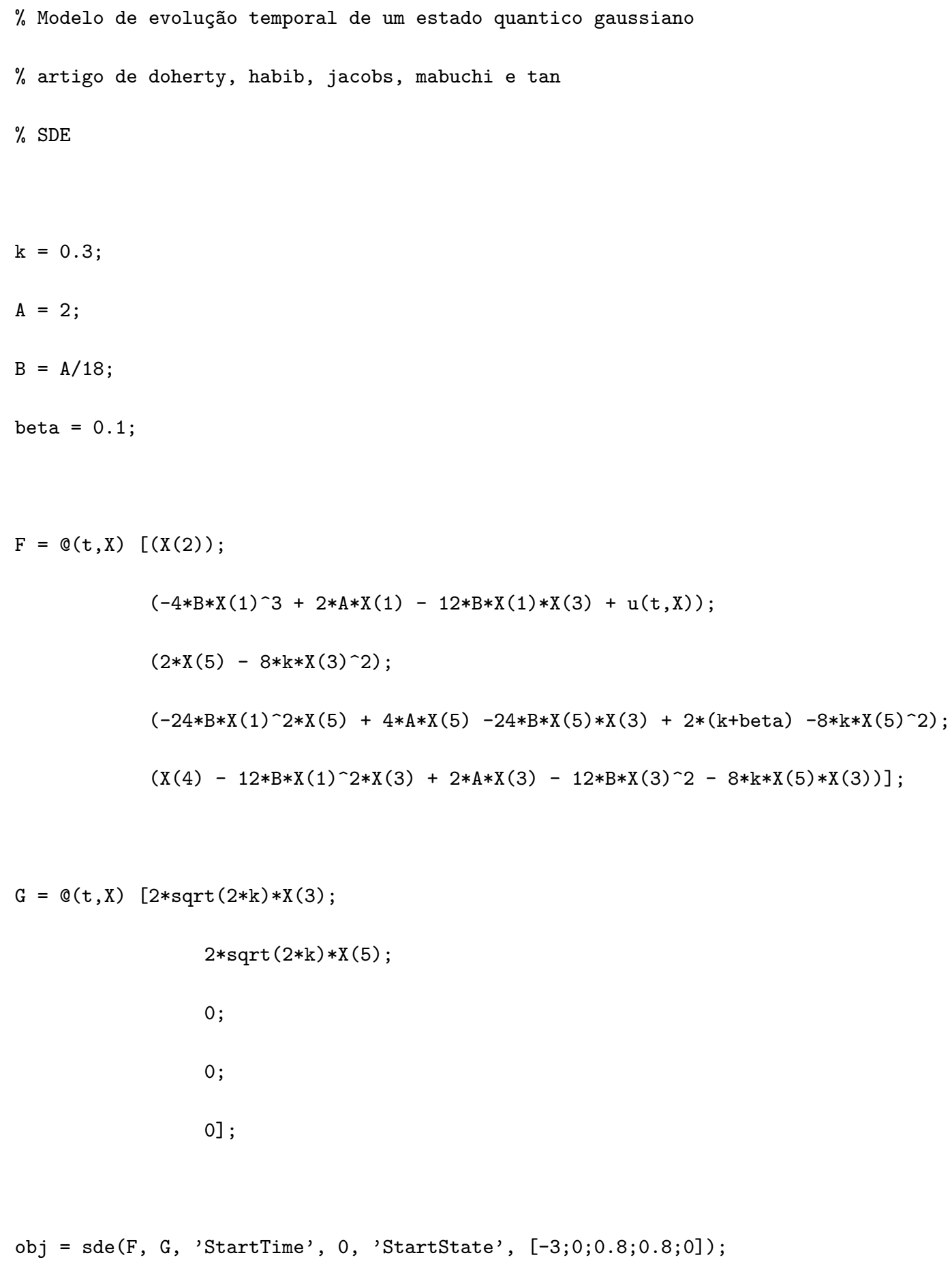


steps $=2 * 100000$;

delta $=0.0001$

global ucontrol

global step;

ucontrol $=0:$ delta: delta*steps;

step $=1$

[paths, times, z] = simByEuler (obj, steps, 'DeltaTime', delta);

$\%$ plot (times, paths)

figure $\%$ new figure

$\operatorname{ax} 1=\operatorname{subplot}(3,1,1) ; \%$ top subplot

$\operatorname{ax} 2=\operatorname{subplot}(3,1,2) ; \%$ bottom subplot

$\operatorname{ax3}=\operatorname{subplot}(3,1,3)$

plot (ax1,times, paths $(:, 1)$, 'b', times, paths $(:, 2),{ }^{\prime} r$ ')

\%title(ax1, 'Quadraturas')

ylabel (ax1, 'x e p')

legend (ax1, '\langle x \rangle',' \langle p \rangle')

plot $(\operatorname{ax} 2, \operatorname{times}, \operatorname{sqrt}(\operatorname{paths}(:, 3))$, ' $r$ ', times, $\operatorname{sqrt}(\operatorname{paths}(:, 4))$, 'g', times, paths $(:, 5)$, 'b', times, paths $(:$ $\%$ title(ax2, 'Incerteza')

ylabel (ax2, '\sigma_x, \sigma_p e C')

legend (ax2, '\sigma_x', '\sigma_p', 'C', '\sigma_x² \sigma_p`2', 'Heisenberg Limit')

plot (ax3, times, ucontrol, 'r');

$\%$ title(ax3, 'Esforço de controle') 
ylabel (ax3, 'u(t) values')

$\%$ legend (ax3, 'u(t)')

Abaixo, encontra-se o código da função de controle, que é chamada pelas funções F e G definidas para determinar a equação diferencial estocástica a ser simulada.

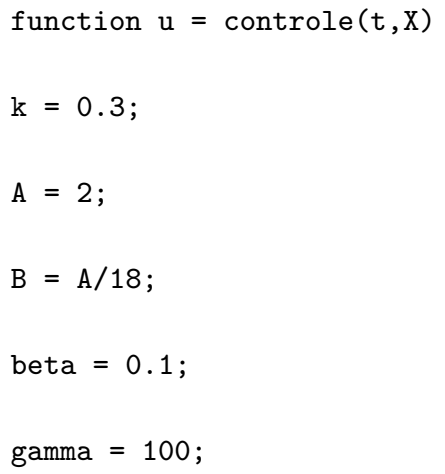


$\mathrm{po}=0 ;$

end

$\mathrm{du}=2 * \mathrm{~A}-12 * \mathrm{~B} * \mathrm{X}(1)^{\sim} 2$;

util $=d u+\operatorname{sqrt}\left(d^{\wedge} 2+\right.$ gamma $) ;$

$\mathrm{u} 0=(2 * \mathrm{~A} * \mathrm{X}(1)-4 * \mathrm{~B} * \mathrm{X}(1)-3) ;$

$\mathrm{u} 1=-\operatorname{util} *(\mathrm{X}(1)-\mathrm{x} 0) ;$

$\mathrm{u} 2=-\operatorname{sqrt}(2 * u t i l+$ gamma $) *(X(2)-\mathrm{p} 0) ;$

$u \operatorname{control}($ step $)=u 0+u 1+u 2 ;$

step $=$ step +1

$\mathrm{u}=\mathrm{u} 0+\mathrm{u} 1+\mathrm{u} 2$

end

Indicamos, na apresentação acima, as quebras de linha por um $\widehat{\text {. }}$

Trata-se de um código muito simples. Praticamente um exemplo de aplicação de uma das funções do pacote de simulações estocásticas da toolbox de finanças. São usadas variáveis globais. 


\section{ANEXO D - Código fonte em QTIP}

Apresentamos a seguir o código-fonte de dois scripts utilizados para simular o sistema de controle por realimentação para geração de estados comprimidos de spin, spin squeezing.

O primeiro script simula uma equação mestra estocástica associada ao processo de medição contínua do observável $J_{z}$. Utilizamos as funções de simulação de equação mestra estocástica configurada para realizar medição no modo detecção homodina.

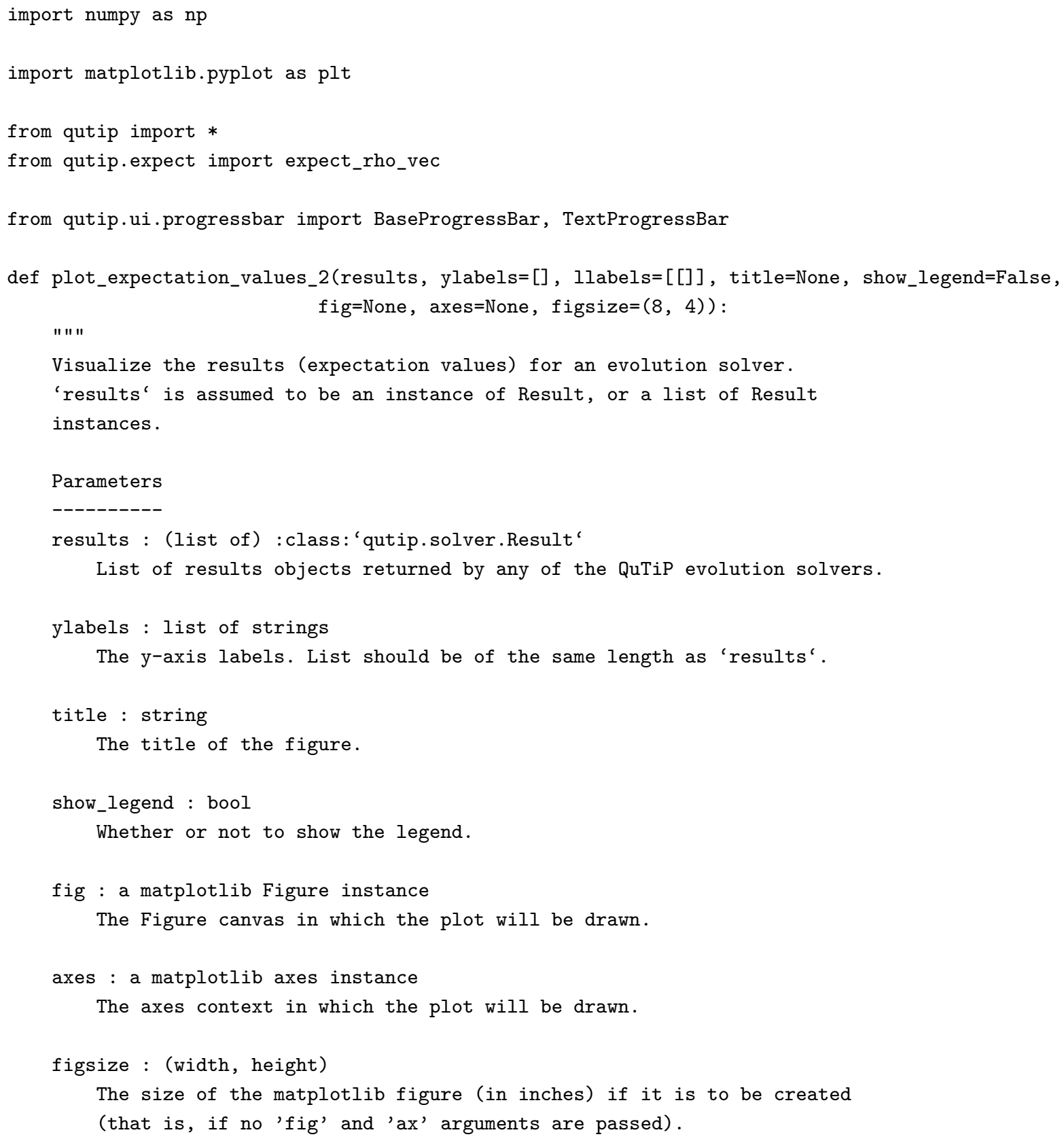


Returns

fig, ax : tuple

A tuple of the matplotlib figure and axes instances used to produce the figure.

" " " "

if not isinstance(results, list):

results $=$ [results $]$

$\mathrm{n}_{-} e_{-}$ops $=\max ([$ len (result.expect $)$ for result in results $\left.]\right)$

if not fig or not axes:

if not figsize:

figsize $=\left(12,3 * n_{-} e_{-}\right.$ops $)$

fig, axes $=$ plt.subplots (n_e_ops, 1 , sharex=True,

figsize=figsize, squeeze $=\mathrm{False}$ )

for $r_{-} i d x$, result in enumerate(results):

for $e_{-} i d x$, e in enumerate(result.expect):

print $r_{-} i d x, e_{-} i d x$

axes [e_idx, 0].plot (result.times, e,

label="\%s" \% (llabels $\left.\left.\left[r_{-} i d x\right]\right)\right)$

if title:

axes $[0,0]$.set_title(title)

axes[n_e_ops - 1, 0].set_xlabel ("time", fontsize=12)

for $n$ in range(n_e_ops):

if show_legend:

axes $[n, 0]$. legend ()

if ylabels:

axes [n, 0] .set_ylabel (ylabels [n] , fontsize=14)

return fig, axes

\#verificar em sakurai 3.8 e pg 220 e 221 a analogia de schwinger e WM 5.7.1

$\mathrm{N}=10 \#$ number of particles

times $=n p . \operatorname{linspace}(0,15,150)$

$[\mathrm{Jx}, \mathrm{Jy}, \mathrm{Jz}]=\operatorname{jmat}(\mathrm{N} / 2.0)$

$[$ en, st $]=\mathrm{Jx} \cdot$ eigenstates ()

psi0 $=$ st $[-1]$ \#estado de maior $\mathrm{J}$ na direcao $\mathrm{x}$

rho0 $=$ psi0*psi0.dag ()

$M=0.1$

\# ME de Wiseman-Milburn pg 261 - eq 5.204

\# averaging and using $\langle\mathrm{dW}\rangle=0$

$\mathrm{H}=\mathrm{Jz}+\mathrm{Jy}$

L0 = liouvillian(None, $[$ np.sqrt $(M) * \mathrm{Jz}])$

C_ops $=[\mathrm{LO}]$ 


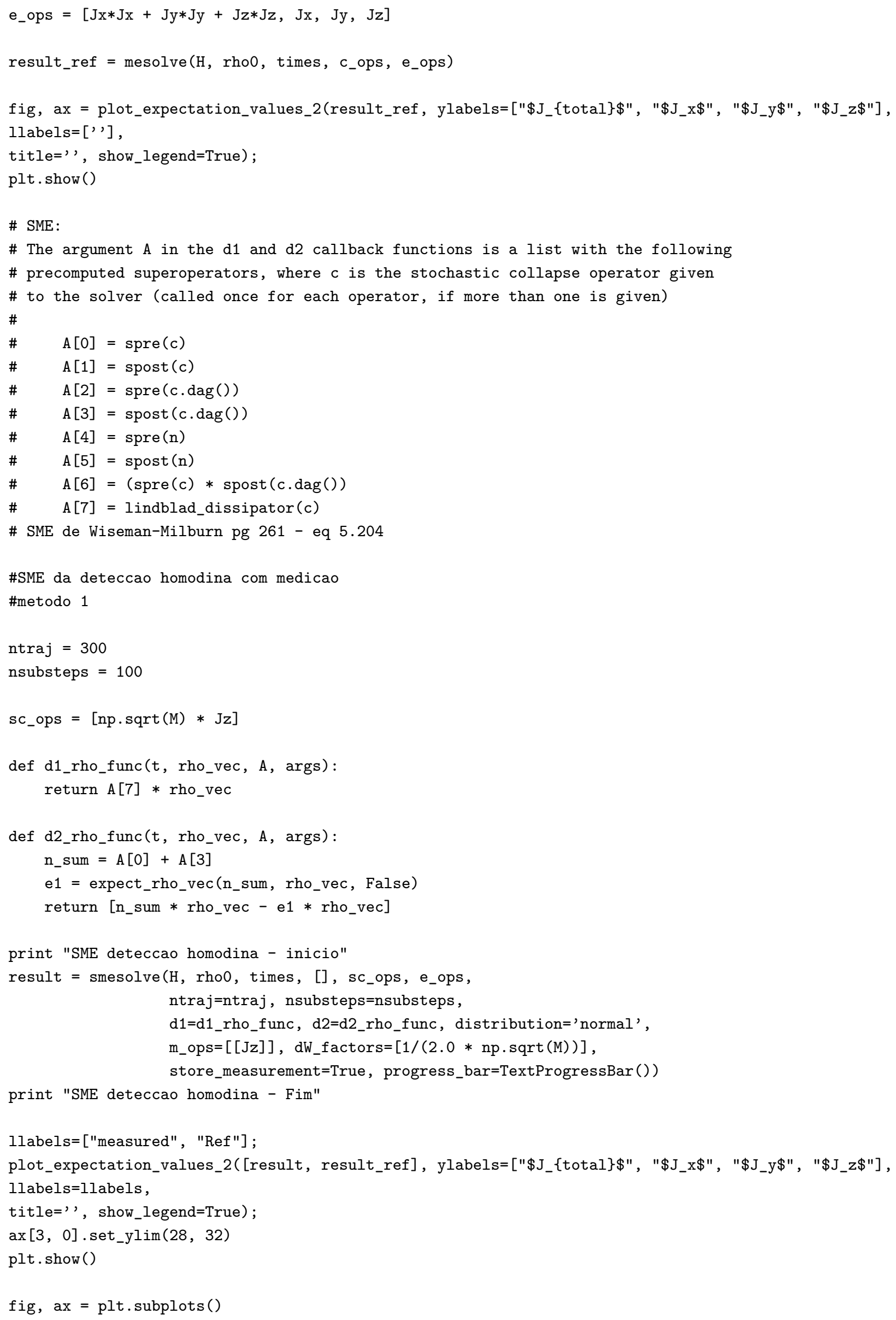




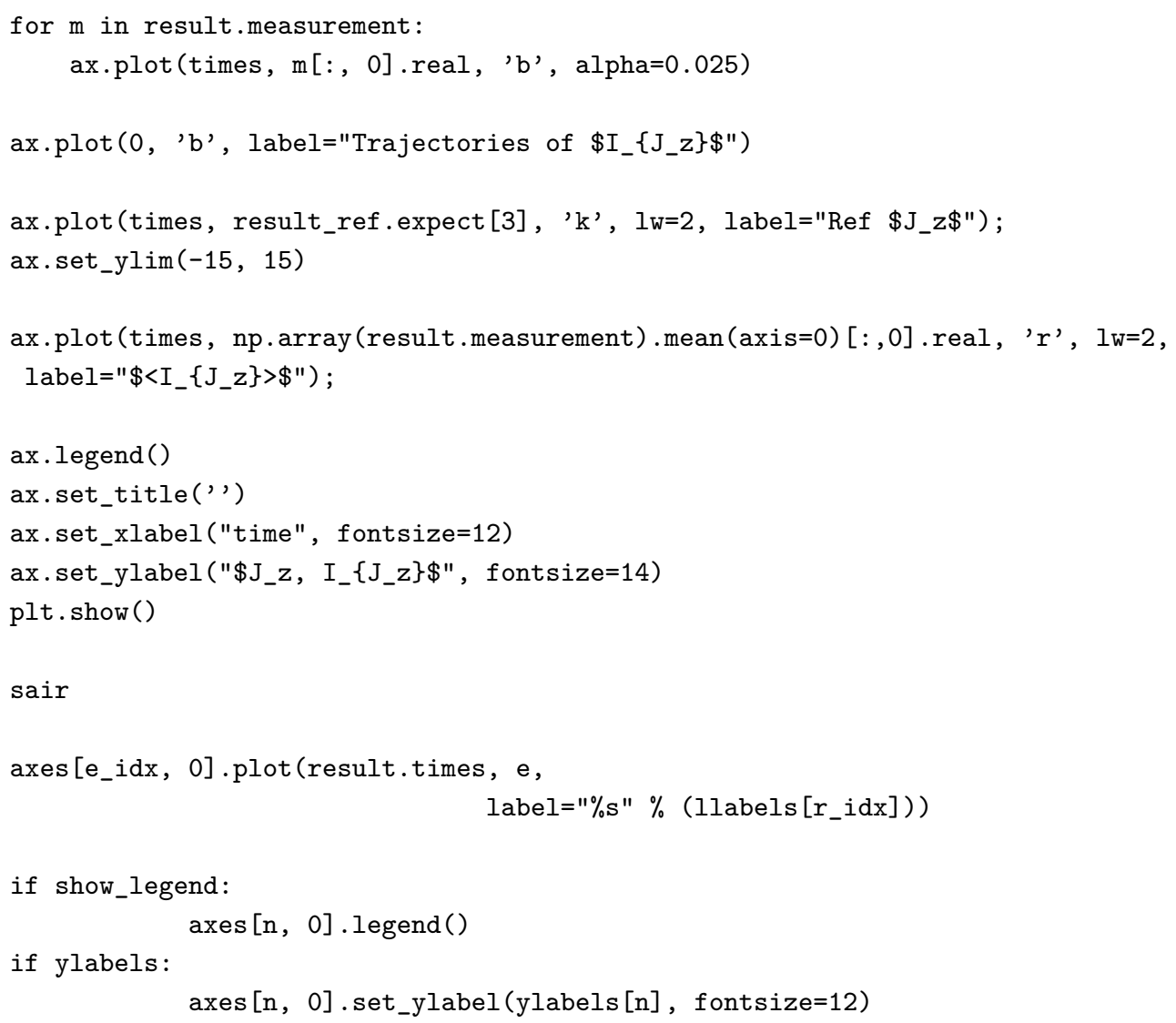

Neste segundo script simulamos uma equação mestra determinística associada ao sistema realimentado. Nesse caso não simulamos as trajetórias quânticas, pois a equação é determinística. O resultado da simulação é o valor esperado para os observáveis no período de tempo em que o sistema é simulado. Nos testes realizados, constatamos que o sistema é bastante sensível a variações na função que determina o parâmetro de realimentação, que define o controle. Para períodos longos de tempo notamos que os valores dos parâmetros se tornam instáveis. Não conseguimos determinar se a fonte dessa instabilidade está nas próprias equação simuladas, em consequência de aproximações locais que têm validade para apenas uma faixa das variáveis de estado, ou se a fonte da instabilidade se encontra no código manualmente desenvolvido para implementar as equações, ou ainda nas bibliotecas de funções do pacote QTIP.

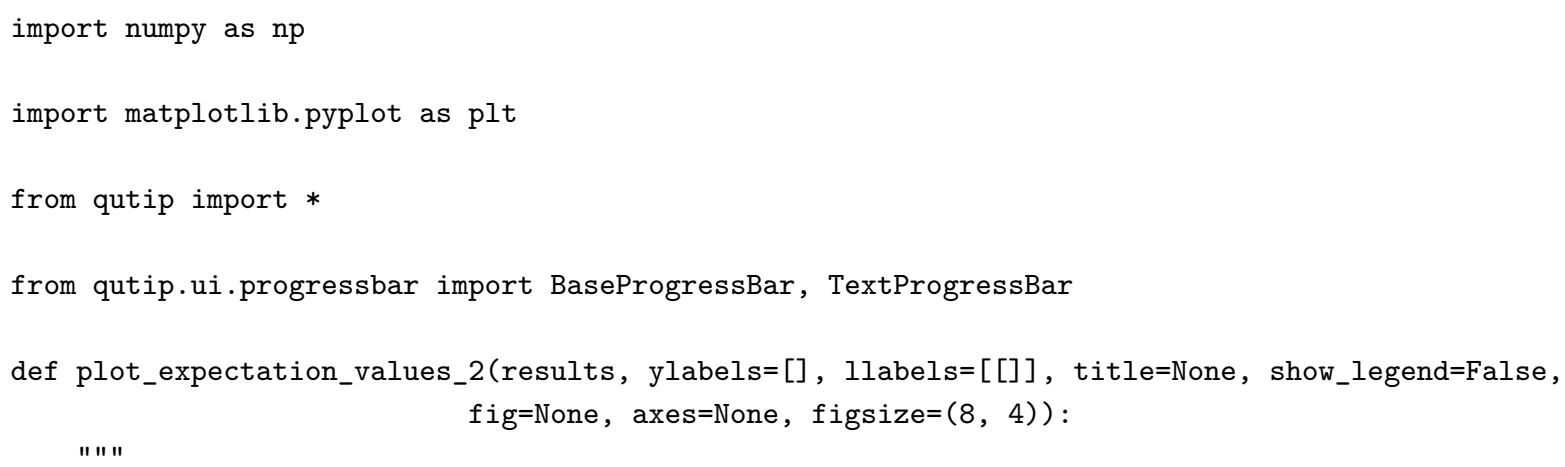


Visualize the results (expectation values) for an evolution solver.

'results' is assumed to be an instance of Result, or a list of Result

instances.

\section{Parameters}

----------

results : (list of) :class: 'qutip.solver.Result'

List of results objects returned by any of the QuTiP evolution solvers.

ylabels : list of strings

The y-axis labels. List should be of the same length as 'results'.

title : string

The title of the figure.

show_legend : bool

Whether or not to show the legend.

fig : a matplotlib Figure instance

The Figure canvas in which the plot will be drawn.

axes : a matplotlib axes instance

The axes context in which the plot will be drawn.

figsize : (width, height)

The size of the matplotlib figure (in inches) if it is to be created

(that is, if no 'fig' and 'ax' arguments are passed).

\section{Returns}

fig, ax : tuple

A tuple of the matplotlib figure and axes instances used to produce

the figure.

" " "

if not isinstance(results, list):

results $=[$ results $]$

$\mathrm{n}_{-} \mathrm{e}_{-} \mathrm{ops}=\max ([\operatorname{len}($ result. expect $)$ for result in results $])$

if not fig or not axes:

if not figsize:

figsize $=\left(12,3 * n_{-} e_{-}\right.$ops $)$

fig, axes $=$ plt.subplots (n_e_ops, 1 , sharex=True, figsize=figsize, squeeze=False)

for $r_{-} i d x$, result in enumerate(results):

for $e_{-} i d x$, e in enumerate(result.expect):

print $r_{-} i d x, e_{-} i d x$

axes $\left[e_{-} i d x, 0\right] \cdot p l o t$ (result.times, e,

label="\%s" \% (llabels $\left.\left.\left[e_{-} i d x\right]\right)\right)$

if title:

axes $[0,0]$.set_title(title)

axes[n_e_ops - 1, 0].set_xlabel ("time", fontsize=12)

for $\mathrm{n}$ in range(n_e_ops):

if show_legend: 


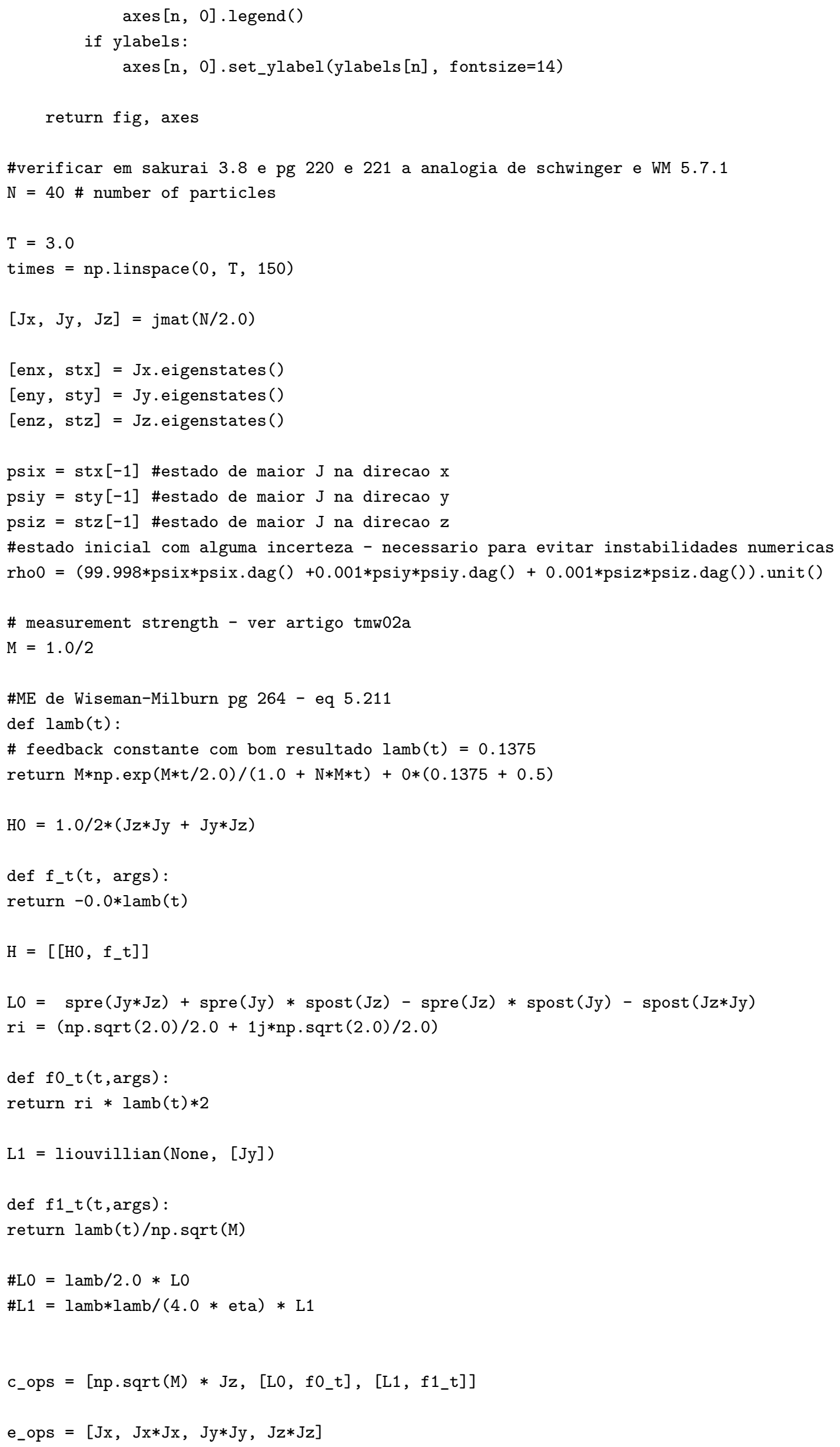




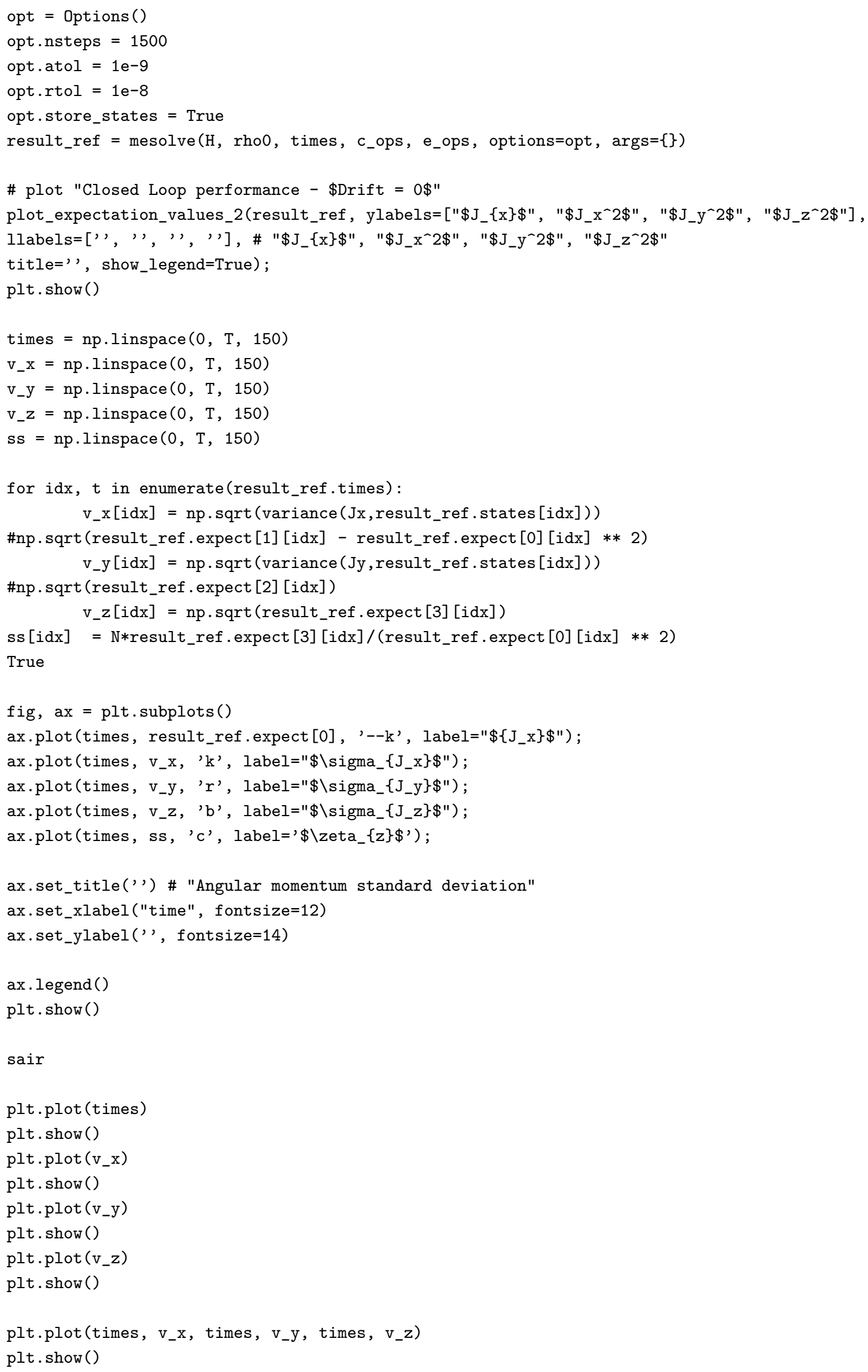




\section{Índice}

elementos textuais, 196 